\title{
Interfacial Properties of Amphiphilic Dendritic Polymers
}

\author{
by \\ Gabriel N. Njikang
}

\author{
A thesis \\ presented to the University of Waterloo \\ in fulfillment of the \\ thesis requirement for the degree of \\ Doctor of Philosophy \\ in \\ Chemistry
}

Waterloo, Ontario, Canada, 2006

(C) Gabriel N. Njikang 2006 
I hereby declare that I am the sole author of this thesis. This is a true copy of the thesis, including any required final revisions, as accepted by my examiners.

I understand that my thesis may be made electronically to the public 


\begin{abstract}
The self-assembly behavior of arborescent polystyrene-graft-poly(ethylene oxide) copolymers (PS-g-PEO) at the air-water interface and the solubilization/release properties of arborescent polystyrene-graft-poly(2-vinylpyridine) (PS-g-P2VP) copolymers were investigated. These amphiphilic dendritic molecules are covalently bonded unimolecular micelles incorporating a highly branched hydrophobic polystyrene core surrounded by a hydrophilic poly(ethylene oxide) or poly(2-vinylpyridine) shell. Molecules of PS-g-PEO copolymers spontaneously formed supramolecular assemblies at the air-water interface. The type of superstructures formed was found to depend upon copolymer composition, while the level of association was more directly related to the branching density of the polymers. At low surface pressures the PEO segments apparently remained adsorbed on the water subphase, but desorbed into water at very high surface pressures, in the condensed monolayer state. Controlled degradation of the PEO chains with UV light greatly enhanced molecular association, resulting in the formation of either large clusters or long ribbon-like superstructures. The PS-g-P2VP copolymers were found to efficiently solubilize and release hydrophobic small molecules in aqueous media. The partition coefficient and solubilization capacity of the copolymers for hydrophobic polyaromatic hydrocarbons increased with the polystyrene content of the copolymers, while the rate of solubilization decreased with increasing branching functionality of the copolymers. The release profiles for two model drugs displayed an initial burst in release followed by gradual approach to equilibrium. The diffusion coefficients of the drugs in the micelles increased with the branching functionality and the generation number of the micelles, presumably due to increased electrostatic repulsions of the protonated vinylpyridine units.
\end{abstract}




\section{Acknowledgements}

I would like to express my sincere gratitude to Professor Mario GAUTHIER. His constant encouragement, guidance, and support have been instrumental in the completion of this thesis.

I would like to thank my supervisory committee members, Professors Christine MORESOLI, Jean DUHAMEL, and Susan MIKKELSEN for their advice and useful suggestions throughout the course of the program.

I would also like to thank my fellow colleagues, Abdul MUNAM, Batiste BOIT, Jason DOCKENDORFF, Oliver NGUON, Nagin PATEL, Shahla ALIAKBARI, Steve TEERTSTRA, Zhongshun YUAN, and the members of the DUHAMEL group for their useful suggestions and discussions.

In addition, I am grateful to Andy MAK for his help with the AFM work, Dale WEBER for helping with TEM, as well as Lan CAO and Jieming LI for the synthesis of most of the polymers used in this work.

Last but not least, I would like to acknowledge the International Council for Canadian Studies (ICCS), the Natural Sciences and Engineering Research Council (NSERC), and the University of Waterloo for their financial support. 
To Antonia B. NJIKANG

for her love, care, support, and understanding. 


\section{Table of Contents}

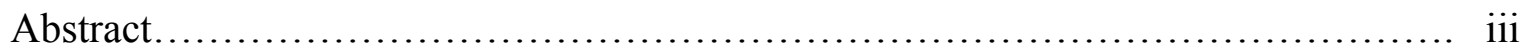

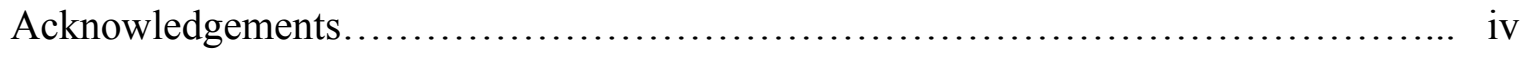

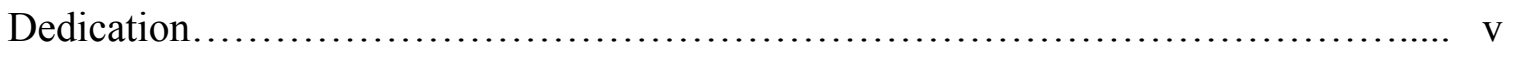

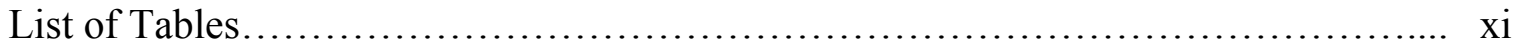

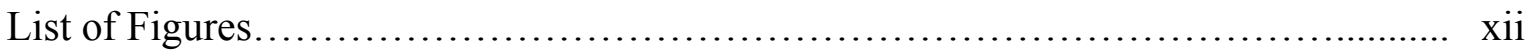

List of Schemes............................................................ xvii

List of Acronyms and Symbols................................................ xviii

Chapter 1 General Introduction............................................ 1

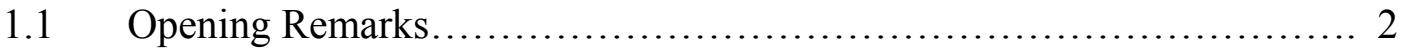

1.2 Research Objectives............................................... 2

$1.3 \quad$ Thesis Outline..................................................... 3

$1.4 \quad$ References.......................................................... 4

Chapter 2 Literature Review.............................................. 5

$2.1 \quad$ Introduction...................................................... 6

2.2 Dendritic Polymers.................................................... 6

2.2.1 Dendrimers..................................................... 8

2.2.2 Dendrigraft Polymers..................................... 10

2.2.3 Hyperbranched Polymers................................... 12

2.3 Amphiphilic Dendritic Polymers.................................... 14

2.3.1 Functionalization of Chain Ends............................ 15

2.3.2 Coupling of Dendritic Substrate with Polymer Chains............. 16

2.3.3 Dendritic-linear Molecules.................................... 17

2.4 Behavior of Amphiphilic Dendritic Polymers at the Air-water Interface... 18

2.4.1 The Langmuir-Blodgett Technique............................ 19

2.4.2 The Langmuir Isotherm.................................... 19

2.4.3 Conformation of Dendritic Amphiphilic Polymers at the Air-water Interface............................................... 20 
2.4.4 Langmuir-Blodgett Film Topology of Amphiphilic Dendritic

Polymers by Atomic Force Microscopy.................... 31

2.5 Solubilization and Encapsulation............................... 33

2.5.1 Host-guest Interactions............................... 33

2.5.1.1 Hydrophobic Interactions........................... 34

2.5.1.2 Physical Entrapment............................. 36

2.5.1.3 Hydrogen Bonding............................... 37

2.5.1.4 Electrostatic Interactions......................... 39

2.5.2 Influence of Micelle and Probe Structure on Encapsulation....... 41

2.5.3 Release of Guest Molecules............................. 44

$2.6 \quad$ Conclusions..................................................... 47

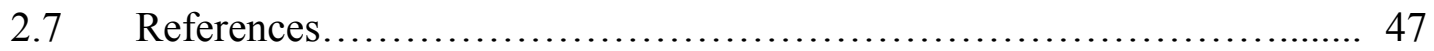

\section{Chapter 3 Self-assembly of Arborescent Polystyrene-graft-Poly(ethylene oxide)}

Copolymers at the Air-water Interface....................... 55

$3.1 \quad$ Abstract.................................................. 56

$3.2 \quad$ Introduction................................................... 57

$3.3 \quad$ Experimental Section....................................... 58

3.3.1 Materials.............................................. 58

3.3.2 Langmuir Trough Experiments................................ 59

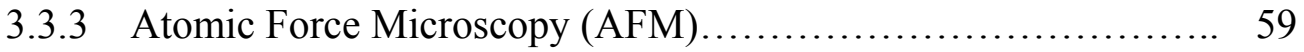

$3.4 \quad$ Results and Discussion........................................ 60

3.4.1 Arborescent PS-g-PEO Copolymers....................... 61

3.4.2 Topology of Langmuir-Blodgett Films.................... 66

3.4.2.1 G1-30PS-LB Samples........................... 67

3.4.2.2 G1-30PS-HB Samples............................ 69

3.4.2.3 Other Samples................................... 71

3.4.2.4 Comparison with Related Polymeric Amphiphiles......... 75

3.4.2.5 Association Mechanism............................. 78

3.5 Conclusions................................................. 79

3.6 Acknowledgements........................................ 80 


\section{Chapter 4 Induced Association of Arborescent Polystyrene-graft-Poly(ethylene} oxide) Copolymers at the Air-water Interface..................... 84

$4.1 \quad$ Abstract......................................................... 85

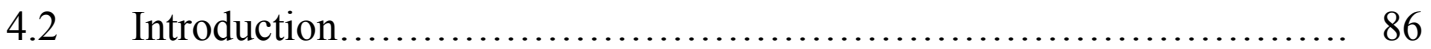

$4.3 \quad$ Experimental Section......................................... 87

$4.4 \quad$ Results and Discussion.......................................... 88

4.4.1 Differential Scanning Calorimetry........................... 89

4.4.2 Langmuir Monolayers.................................. 92

4.4.3 Influence of Copolymer Structure on the Compression Isotherms.. 93

4.4.4 Surface Compressional Modulus................................. 96

4.4.5 Conformation of Arborescent PS-g-PEO Copolymers at the Air-water Interface.................................. 98

4.4.6 Influence of Compression on the Topology of Langmuir-Blodgett Films of High PEO Content Samples............................ 104

4.4.7 Temperature Dependence of Langmuir-Blodgett Film Topology for Samples with High PEO Contents....................... 108

4.4.8 Topology of Langmuir-Blodgett Films Obtained Upon UV Irradiation of the Sample Solutions........................ 111

4.4.9 Association Behavior of Copolymers with Low PEO Contents..... 118

4.5 Conclusions....................................................... 121

4.6 Acknowledgements......................................... 122

$4.7 \quad$ References..................................................... 122

Chapter 5 Arborescent Polystyrene-graft-Poly(2-vinylpyridine) Copolymers as Unimolecular Micelles: Solubilization Studies..................... 127

$5.1 \quad$ Abstract........................................................ 128

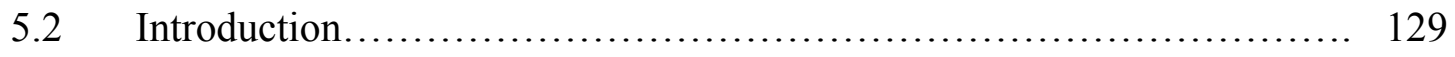

$5.3 \quad$ Experimental Section......................................... 131

5.3.1 Sample Preparation..................................... 131 
5.3.2 Fluorescence and UV Measurements........................ 132

$5.4 \quad$ Results and Discussion............................................... 134

5.4.1 Equilibrium Probe Uptake.................................... 136

5.4.2 Micellar Uptake Kinetics..................................... 139

5.4.3 Determination of Partition Coefficient and Solubilization Capacity 141

5.4.4 Solubilization of Highly Hydrophobic Probes..................... 142

5.4.5 Solubilization of Naphthalene and Derivatives................... 145

5.4.6 Location of Probes in the Micelles.................................. 146

5.4.7 Additional Evidence in Support of Selective Sorption.............. 148

5.5 Conclusions...................................................... 152

5.6 Acknowledgements................................................. 152

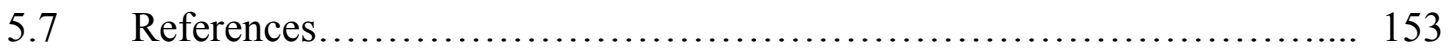

Chapter 6 Sustained Release Properties of Arborescent Polystyrene-graft-Poly(2vinylpyridine) Copolymers....................................... 157

$6.1 \quad$ Abstract............................................................... 158

6.2 Introduction........................................................ 159

$6.3 \quad$ Experimental Section............................................ 161

6.3.1 Materials................................................ 161

6.3.2 Sample Preparation.......................................... 161

6.3.3 Fluorescence Studies.................................... 162

6.3.4 Loading and Release of Indomethacin and Lidocaine............ 163

$6.4 \quad$ Results and Discussion................................................ 163

6.4.1 Nonradiative Energy Transfer............................... 164

6.4.2 Fluorescence Quenching Experiments........................ 167

6.4.3 In vitro Release Kinetics of Indomethacin and Lidocaine.......... 174

6.5 Conclusions....................................................... 183

$6.6 \quad$ Acknowledgements........................................... 184

$6.7 \quad$ References.......................................................... 184

Chapter 7 General Conclusions and Suggestions for Future Work.............. 188 
7.1 General Conclusions.......................................... 189

7.2 Suggestions for Future Work..................................... 190

7.2.1 Behavior of Arborescent PS-g-P2VP Copolymers at the Air-water Interface....................................... 191

7.2.2 Aggregation of Arborescent PS-g-P2VP Copolymers in Aqueous Solutions............................................................ 192 


\section{List of Tables}

Table 2.1 Experimental and Theoretical Molecular Areas Calculated as a Function of Generation Number for Modified Polypropylenimine Dendrimers............ 22

Table 2.2 Comparison of Idealized and Estimated Number of Alkyl Chains per Molecule for Hyperbranched Polyesters with Alkyl Substitutents............ 30

Table 3.1 Characteristics of Arborescent PS- $g$-PEO Copolymers Used................ 64

Table 4.1 Characteristics of Arborescent Copolymers Used........................ 90

Table 4.2 Crystallinity of PEO and Phase Transition Temperatures for PS- $g$-PEO Copolymers.................................................... 91

Table 4.3 Surface Compression Moduli for the Gaseous, Liquid, and Solid States of Arborescent PS-g-PEO Copolymer Monolayers........................ 97

Table 4.4 Molecular Diameters and Collapse Pressures of Langmuir Monolayers...... 100

Table 5.1 Characteristics of Arborescent PS-g-P2VP Copolymers Used.............. 136

Table 5.2 Partition Coefficient $K_{\mathrm{w}}$ for PAHs................................... 143

Table 5.3 Physical Properties of PAHs......................................... 144

Table 5.4 Solubilization Capacity $\kappa^{G}$ (mg probe/g polymer) for PAHs.............. 145

Table 6.1 Characteristics of PS-g-P2VP Arborescent Copolymers Used in the Study .................................................... 164

Table 6.2 Lifetimes (ns) and Preexponential Factors Obtained from Analysis of Decays .................................................. 171

Table 6.3 Quenching Data for Pyrene-Loaded Arborescent PS-g-P2VP.............. 173

Table 6.4 Parameters $k$ and $n$ of the Power Law Model for the Release of Indomethacin from PS- $g$-P2VP Copolymers........................... 180

Table 6.5 Diffusion Coefficients of Indomethacin in Arborescent PS-g-P2VP Copolymers............................................... 183 


\section{List of Figures}

Figure 2.1 Structure of dendritic polymers: Dendrimer, dendrigraft polymer, and hyperbranched polymer.

Figure 2.2 Dependence of intrinsic viscosity on molecular weight for linear

polymers, hyperbranched polymers, and dendrimers.

Figure 2.3 Dependence of intrinsic viscosity on molecular weight for arborescent polystyrenes of different structures.

Figure 2.4 Polypropylenimine dendrimers modified with alkyl chains, and three types of hydrophobic chains used to modify the surface of the hydrophilic dendrimers.

Figure 2.5 Langmuir isotherms for different generations of functionalized polypropylenimine dendrimers at $25^{\circ} \mathrm{C}$

Figure 2.6 Amphiphilic dendrimers of different generations bearing fullerene units, and Langmuir isotherms of $G 1, G 2$, and $G 3$ amphiphillic dendrimers at $20^{\circ} \mathrm{C}$

Figure 2.7 Diblock dendrimer with two hemispherical dendrons, and four successive compression and decompression cycles showing minimal hysteresis...

Figure 2.8 Structure of HA-PAMAM dendrimers and their surface pressure and surface potential isotherms at the air-water interface.

Figure 2.9 Face-on and edge-on configurations of disk-like molecules in Langmuir and Langmuir-Blodgett films....

Figure 2.10 Langmuir compression isotherms for HP-0, HP-10, HP-25, HP-50, and HP-75 at the air/water interface

Figure 2.11 Topographical and phase AFM images for Langmuir-Blodgett films of HP-25, HP-50, and HP-75 obtained at a surface pressure of $35 \mathrm{mN} / \mathrm{m}$ 32

Figure 2.12 Structure of dendritic box proposed by Meijer, and cleavage reactions leading to selective liberation of dyes from the dendritic box 
Figure 2.13 Hydrogen bonding interactions between phenanthrenequinone

and terminal phenyl-urea group of dendrimer,

phenyl-urea terminated dendrimer, and cyclic voltammetry

curve for phenanthrenequinone in DMF........................... 38

Figure 2.14 Structure of modified PAMAM dendrimers used as hosts,

and biological guest molecules

Figure 2.15 Cumulative release of free lidocaine and polymer-encapsulated

lidocaine showing complete release of encapsulated drug

Figure 2.16 Cumulative profiles for the release of ibuprofen. Release of pure drug,

drug in $G 4$ dendrimer, and drug in $G 3$ dendrimer, all in culture medium;

drug in $G 4$ dendrimer in deionized water, drug in $G 4$ dendrimer

in methanol

Figure 3.1 AFM amplitude images for copolymer G1-5PS-HB-74

deposited on mica, and silicon

Figure 3.2 Height and amplitude images for Langmuir-Blodgett films for copolymers

G1-30PS-LB-15, G1-30PS-LB-22, and G1-30PS-LB-31

Figure 3.3 Height and amplitude AFM images for copolymers G1-30PS-HB-8, G1-30PS-HB-24, and G1-30PS-HB-43.

Figure 3.4 Height and amplitude AFM micrographs obtained for copolymer $G 1$-14PS-LB-19

Figure 3.5 AFM height and amplitude images for copolymer $G 1-5 \mathrm{PS}-\mathrm{HB}-74$

Figure 3.6 Amplitude AFM images for Langmuir-Blodgett films obtained from copolymer G3-5PS-HB-43

Figure 4.1 DSC thermograms for copolymer G1-30PS-LB-22 and the parent polystyrene core (G1-30PS-LB)

Figure 4.2 Langmuir compression isotherms for copolymers G1-30PS-LB-6.5, G1-30PS-LB-15, G1-30PS-LB-22, and G1-30PS-LB-31 95

Figure 4.3 Langmuir compression isotherms for copolymers G1-30PS-HB-8, 
G1-30PS-HB-24, G1-30PS-HB-33, and G1-30PS-HB-43.............. 95

Figure 4.4 Langmuir compression isotherm for copolymer G3-5PS-HB-43......... 96

Figure 4.5 Calculation of limiting area per molecule and area per molecule in the liquid phase using copolymer G1-30PS-LB-31 as an example....... 98

Figure 4.6 Bearing analysis on a $1.5 \times 1.5 \mu \mathrm{m}^{2}$ box area showing the monolayer thickness for copolymer G1-30HB-43 at $0 \mathrm{mN} / \mathrm{m}, 4 \mathrm{mN} / \mathrm{m}$, and $20 \mathrm{mN} / \mathrm{m} \ldots 103$

Figure 4.7 Influence of pressure on the self-assembly of high PEO content, low branching functionality copolymers: AFM images for Langmuir-Blodgett monolayers for G1-30PS-LB-22 copolymers

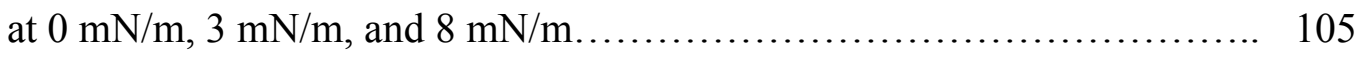

Figure 4.8 Influence of pressure on the self-assembly of high PEO content, high branching functionality copolymers: AFM images of Langmuir-Blodgett monolayers for G1-30PS-HB-24 copolymers at $0 \mathrm{mN} / \mathrm{m}, 4 \mathrm{mN} / \mathrm{m}$, and $9 \mathrm{mN} / \mathrm{m}$

Figure 4.9 AFM pictures for copolymers G1-30PS-LB-22, and G1-30PS-HB-24, after decompression to $0 \mathrm{mN} / \mathrm{m}$.

Figure 4.10 Top view of the reversible association model proposed for the reversible formation of ribbon-like superstructures by $\mathrm{HB}$, high PEO content samples.. 107

Figure 4.11 Film topology of copolymer $G 1-30 \mathrm{PS}-\mathrm{HB}-43$ at $\mathrm{T}=12{ }^{\circ} \mathrm{C}$, and $\mathrm{T}=37^{\circ} \mathrm{C}$ 108

Figure 4.12 Effect of temperature on compression isotherms for copolymer G1-30PS-HB-43 at $10.5,12,17^{\circ} \mathrm{C}$, and $22,27,32,37^{\circ} \mathrm{C}$ 110

Figure 4.13 Changes in film topology for copolymer G1-30PS-HB-43 upon aging of solution under ambient conditions for 7 days, 20 days, 34 days, 44 days, 110 days, and 130 days

Figure 4.14 Influence of UV irradiation on Langmuir-Blodgett film topology for copolymer G1-30PS-HB-43 after solution irradiation at $50 \mathrm{~cm}$ with $6 \mathrm{UV}$ lamps for $0 \mathrm{~min}, 2.5 \mathrm{~min}, 5 \mathrm{~min}, 10 \mathrm{~min}, 15 \mathrm{~min}$, and $30 \mathrm{~min}$ 114

Figure 4.15 AFM pictures for Langmuir-Blodgett films obtained for copolymer G1-30PS-HB-43 after solution irradiation with a single UV lamp for $1 \mathrm{~h}, 2 \mathrm{~h}, 4 \mathrm{~h}, 6 \mathrm{~h}, 9 \mathrm{~h}$, and $13 \mathrm{~h}$. 
Figure 4.16 Influence of linear PEO contaminant on Langmuir-Blodgett film topology for copolymer G1-30PS-HB-43/PEO: 0\% w/w PEO, 5\% w/w PEO, 7\% w/w PEO, 14\% w/w PEO, and 29\% w/w PEO

Figure 4.17 Influence of pressure on the self-assembly of low PEO content copolymers:

Height and amplitude images for $G 1-30 \mathrm{PS}-\mathrm{LB}-15$ at $0 \mathrm{mN} / \mathrm{m}$, and $2 \mathrm{mN} / \mathrm{m}$....

Figure 4.18 Influence of solution aging on the self-assembly of low PEO content copolymers: Amplitude micrographs of Langmuir-Blodgett films for G1-30PS-LB-15 after aging of the solution for 35 days 121

Figure 5.1 Structures of polyaromatic hydrocarbons used......................... 132

Figure 5.2 Structure of arborescent PS-P2VP5 copolymers of different generations..... 135

Figure 5.3 UV equilibrium absorption spectra for PyM solubilized by arborescent copolymer samples...

Figurer 5.4 Time dependence of PyM uptake by arborescent micelles. 139

Figure 5.5 Analysis of micellar uptake kinetics. 140

Figure 5.6 Composition dependence of partition coefficient for Py, PyM, and NapMe.

Figure 5.7 Red shift of UV absorbance of PyM in P2VP30 and P2VP5 samples. 149

Figure 6.1 Three generations of arborescent PS- $g$-P2VP copolymers 161

Figure 6.2 Emission spectrum for pyrene excited at $344 \mathrm{~nm}$, overlap with absorption spectrum for perylene, and resulting emission spectrum for perylene

Figure 6.3 Normalized perylene intensity ratios $R_{\mathrm{A}}$ for $G 1, G 2$, and $G 3$ arborescent copolymers.

Figure 6.4 Stern-Volmer and modified Stern-Volmer plots for pyrene-loaded $G 1, G 2$, and $G 3$ PS- $g$-P2VP copolymers

Figure 6.5 Decay curve for pyrene in $G 1$ arborescent PS- $g$-P2VP copolymer........ 170

Figure 6.6 Structures of indomethacin and lidocaine. 175

Figure 6.7 Release profile for indomethacin and lidocaine in $0.05 \mathrm{M} \mathrm{HCl}$ in the free state and from $G 1$ PS- $g$-P2VP copolymer. 176

Figure 6.8 Percentage mass fraction of indomethacin release from $G 1, G 2$, 
$G 3$ copolymers, and lidocaine released from the $G 1$ copolymer

Figure 6.9 Influence of initial indomethacin loading (w/w) on release from G1 micelles: $29 \%, 33 \%$, and $46 \%$ loading

Figure 7.1 AFM micrographs for Langmuir-Blodgett films of arborescent PS-g-P2VP copolymers before compression: G1PS-g-P2VP, $f_{\mathrm{w}}=630, G 1 \mathrm{PS}-\mathrm{g}-\mathrm{P} 2 \mathrm{VP}, f_{\mathrm{w}}=370$

Figure 7.2 Change in micellar aggregate size with decreasing $\mathrm{HCl} / 2 \mathrm{VP}$ mole ratio $R_{\mathrm{m}}$

Figure 7.3 Dependence of average diameter of large compound micelles on $\mathrm{HCl} / 2 \mathrm{VP}$ mole ratio $R_{\mathrm{m}}$ 


\section{List of Schemes}

Scheme 3.1 Reaction scheme for the synthesis of arborescent PS- $g$-PEO copolymers.... 62 


\section{List of Acronyms and Symbols}

$\begin{array}{ll}2 \text { VP } & \text { 2-vinylpyridine } \\ \alpha & \text { degree of crystallization } \\ \delta & \text { shell thickness } \\ \kappa^{\mathrm{G}} & \text { solubilization capacity } \\ \pi & \text { surface pressure } \\ \pi_{\mathrm{c}} & \text { collapse surface pressure } \\ \rho & \text { density } \\ \tau_{0} & \text { fluorescence lifetime } \\ \tau_{\mathrm{D}} & \text { donor decay constant } \\ <\tau>0 & \text { average fluorescence lifetime } \\ \gamma & \text { surface tension of monolayer-covered subphase } \\ \gamma_{0} & \text { surface tension of pure subphase } \\ {[Q]} & \text { quencher concentration } \\ {[\eta]} & \text { intrinsic viscosity } \\ A & \text { surface area } \\ A_{0} & \text { limiting area per molecule } \\ \text { AFM } & \text { atomic force microscopy } \\ A_{\mathrm{L}} & \text { molecular area from the liquid phase } \\ \text { An } & \text { anthracene } \\ \text { ATRP } & \text { atom transfer radical polymerization } \\ \text { bp } & \text { boiling point } \\ \text { CMC } & \text { critical micelle concentration } \\ C_{\mathrm{s}} & \text { compressibility } \\ C_{\mathrm{s}}^{-1} & \text { surface compressional modulus } \\ C_{m i c}^{G} & \text { micelle concentration } \\ D & \text { average diffusion coefficient } \\ D_{0} & \text { limiting diameter } \\ \mathrm{DB} & \text { degree of branching } \\ & \end{array}$




$\begin{array}{ll}\text { DCC } & \text { dicyclohexylcarbodiimide } \\ D_{\mathrm{h} \text { PEO }} & \text { hydrodynamic diameter of poly(ethylene oxide) chain } \\ D_{\mathrm{h}} & \text { hydrodynamic volume } \\ D_{\mathrm{i}} & \text { initial diffusion coefficient } \\ D_{\mathrm{L}} & \text { molecular diameter in the liquid-like phase } \\ \text { DLS } & \text { dynamic light scattering } \\ \text { DMAP } & \text { dimethylaminopyridine } \\ \text { DMF } & \text { N, } N \text { - dimethylformamide } \\ \text { DSC } & \text { differential scanning calorimetry } \\ f_{\mathrm{a}} & \text { fraction of pyrene accessible to quencher } \\ \text { FRET } & \text { fluorescence resonance energy transfer } \\ \text { FTIR } & \text { fourier transform infrared } \\ f_{\mathrm{w}} & \text { branching functionality } \\ G & \text { generation } \\ \mathrm{g}(\tau) & \text { correlation function } \\ \text { HA } & \text { 12-hydroxydodecanoic acid } \\ \text { HB } & \text { high branching } \\ I & \text { fluorescence intensity } \\ I_{0} & \text { unquenched fluorescence intensity } \\ I_{1} & \text { pyrene emission intensity at a wavelength of } 373 \mathrm{~nm} \\ I_{3} & \text { pyrene emission intensity at a wavelength of } 383 \mathrm{~nm} \\ I_{\mathrm{A}}(0) & \text { fluorescence intensity of acceptor at time } 0 \\ I_{\mathrm{A}}(\mathrm{t}) & \text { fluorescence intensity of acceptor at time } t \\ I_{\mathrm{A}}(\infty) & \text { fluorescence intensity of acceptor at time } \infty \\ K_{\mathrm{a}} & \text { association or binding constant } \\ K_{\mathrm{ow}} & \text { octane-water partition coefficient } \\ k_{\mathrm{q}} & \text { bimolecular quenching constant } \\ k_{T} & \text { rate constant for energy transfer process } \\ K_{\mathrm{w}} & \text { micelle-water partition coefficient } \\ \text { LB } & \text { low branching } \\ M_{\infty} & \text { cumulative weight release at equilibrium } \\ & \end{array}$




\begin{tabular}{|c|c|}
\hline$M_{w}^{b r}$ & weight-average molecular weight of a side chain (branch) \\
\hline$M_{\mathrm{eq}}$ & cumulative weight release at equilibrium \\
\hline$M_{\mathrm{n}}$ & number-average molecular weight \\
\hline$M_{\mathrm{PEO}}$ & molecular weight of poly(ethylene oxide) segments \\
\hline$M_{\mathrm{t}}$ & cumulative weight release at time $t$ \\
\hline MW & molecular weight \\
\hline$M_{\mathrm{w}}$ & weight-average molecular weight \\
\hline MWCO & molecular weight cut off \\
\hline$N_{\mathrm{A}}$ & Avogadro's constant \\
\hline Nap & naphthalene \\
\hline $\mathrm{NapBr}$ & bromonaphthalene \\
\hline NapMe & methylnaphthalene \\
\hline NMR & nuclear magnetic resonance \\
\hline $\mathrm{P} 2 \mathrm{VP}$ & poly(2-vinylpyridine) \\
\hline $\mathrm{PAH}$ & polyaromatic hydrocarbon \\
\hline PAMAM & polyamidoamine \\
\hline PEG & poly(ethylene glycol) \\
\hline PEO & poly(ethylene oxide) \\
\hline Phe & phenanthrene \\
\hline PS & polystyrene \\
\hline PS-g-P2VP & polystyrene-graft-poly(2-vinylpyridine) \\
\hline PS- $g$-PEO & polystyrene-graft-poly(ethylene oxide) \\
\hline PTFE & polyterafluoroethylene \\
\hline Py & pyrene \\
\hline PyM & 1-pyrenemethanol \\
\hline$q$ & scattering vector \\
\hline$r$ & radius of sphere \\
\hline$R$ & sum of molecular radii of fluorophore and quencher \\
\hline$R_{0}$ & Förster distance \\
\hline$R_{\mathrm{A}}$ & normalized fluorescence intensity ratio \\
\hline$R_{\mathrm{G}}$ & radius of gyration \\
\hline
\end{tabular}




$\begin{array}{ll}R_{\mathrm{H}} & \text { hydrodynamic radius } \\ R_{\mathrm{m}} & \text { HCl/2VP mole ratio } \\ R_{\mathrm{PEO}} & \text { extended chain length of poly(ethylene oxide) } \\ \text { SANS } & \text { small angle neutron scattering } \\ \text { SAXS } & \text { small angle x-ray scattering } \\ \text { SEC } & \text { size exclusion chromatography } \\ S_{s}^{G} & \text { concentration of probe in the solvent } \\ S_{\text {tot }}^{G} & \text { concentration of probe in micellar solution } \\ S_{m i c} & \text { number of moles of probe solubilized per gram of micelle } \\ S_{\mathrm{s}} & \text { number of moles of probe dissolved per gram of solvent } \\ t \text {-BOC } & \text { tert-butyloxycarbonyl } \\ \text { TEM } & \text { transmission electron microscopy } \\ T_{\mathrm{g}} & \text { glass transition temperature } \\ T_{\mathrm{m}} & \text { melting temperature } \\ \mathrm{UV} & \text { ultraviolet light } \\ \mathrm{V}_{\mathrm{h}} & \text { hydrodynamic volume } \\ \text { w/w } & \text { weight for weight }\end{array}$




\title{
Chapter 1
}

\author{
General Introduction
}




\subsection{Opening Remarks}

Dendritic polymers are materials of great interest because of their unique features including a compact globular topology, the presence of internal cavities, and a large number of functional groups at the periphery. ${ }^{1}$ When polar groups are present on their surface, these macromolecules behave in aqueous media like covalently bonded unimolecular micelles characterized by the absence of a critical micelle concentration (CMC). Their ability to retain their overall shape under various solvency conditions makes them interesting candidates for applications such as solubilization, drug delivery, and controlled release.

Arborescent copolymers are a class of dendritic polymers mimicking some of the characteristics of dendrimers on a larger scale. Copolymers incorporating polar chain segments on their surface are synthesized through stepwise anionic grafting reactions, ${ }^{3}$ and behave like unimolecular micelles. Unlike other dendritic amphiphiles, arborescent copolymers are assembled from linear polymer chains rather than small molecule monomers. By this approach a very high molecular weight is attained in very few steps, while control is achieved over the chemical composition and the architecture of the molecules.

\subsection{Research Objectives}

This dissertation focuses on the self-assembly and micellar properties of two families of arborescent amphiphilic copolymers, namely arborescent polystyrene-graft-poly(ethylene oxide) (PS-g-PEO) and arborescent polystyrene-graft-poly(2-vinylpyridine) (PS-g-P2VP) copolymers. The main goals were to study the association behavior of arborescent PS-g-PEO copolymers at the air-water interface, and to investigate the solubilization/release properties of arborescent PS-g-P2VP copolymers in aqueous media. The arborescent PS- $g$-PEO 
copolymers used have a relatively large PS core as compared to the PEO shell, which favors their association at the air-water interface due to significant van der Waals forces between the cores. Arborescent PS-g-P2VP copolymers, on the other hand, are soluble in aqueous media and display little or no association once the 2VP units are protonated. These macromolecular micelles are able to solubilize small molecules within their core and/or corona.

\subsection{Thesis Outline}

The general introduction in this chapter is followed by background information on the interfacial properties of the different families of dendritic polymers, and a literature review on the unique physical properties of these materials. The synthesis of amphiphilic dendritic polymers is also highlighted. This review is meant to be a concise account on the behavior of amphiphilic dendritic copolymers at the air-water interface and their solubilization/release properties.

The next four chapters describe discrete projects relating to the interfacial properties of arborescent copolymers. Each chapter is organized in manuscript format, with an abstract, an introduction on relevant background information, as well as a description of the experimental methods used, a discussion of the results, a summary of conclusions, and a list of references. Chapter 3 focuses on the association behavior of arborescent PS- $g$-PEO copolymers spread at the air-water interface in a Langmuir trough as determined using atomic force microscopy (AFM) measurements on thin films. The influence of copolymer composition and structure on the monolayer topology obtained is discussed. In Chapter 4, the self-assembly of arborescent PS-g-PEO copolymers at the air-water interface is investigated by the same techniques to study the influence of compression, subphase temperature 
variations, and PEO chain degradation on the association behavior of the copolymers. The solubilization of small molecules by arborescent PS-g-P2VP copolymers in aqueous solutions is presented in Chapter 5. The influence of the copolymer generation number and the solute structure on both the rate of solubilization and the solubilization capacity is examined. The influence of solute hydrophobicity on its location within the micelle is also highlighted. In Chapter 6, the release behavior of small molecules from arborescent PS-gP2VP copolymers of different generations in aqueous media is investigated. The power law model and Fick's second law of diffusion are used to model the release kinetics.

Chapter 7 provides overall concluding remarks, a summary of original contributions to knowledge, and suggestions for future work.

\subsection{References}

1. (a) Dykes, G. M. J. Chem. Technol. Biotechnol. 2001, 76, 903. (b) Moorefield, C. N; Newkome, G. R. C. R. Chimie 2003, 6, 715. (c) Gitsov, I.; Lin, C. Curr. Org. Chem. 2005, 9, 1025 .

2. (a) Gauthier, M.; Li, J.; Dockendorff, J. Macromolecules 2003, 36, 2642 (b) Teerststra, S. J.; Gauthier, M. Prog. Polym. Sci. 2004, 29, 277. 
Chapter 2

Literature Review 


\subsection{Introduction}

A considerable amount of interest is currently being devoted to amphiphilic dendritic polymers. ${ }^{1-4}$ These polymers are characterized by either a hydrophobic core with a cascade branched structure, surrounded by a hydrophilic corona or shell, or a highly branched hydrophilic core surrounded by a hydrophobic exterior. Amphiphilic dendritic molecules are best described as covalently bonded unimolecular micelles. Unlike micelles assembled from small molecule and block copolymer amphiphiles, which exist at equilibrium with single (non-associated) molecules, dendritic micelles retain their overall shape under most solvency conditions and display no critical micelle concentration (CMC). This chapter will focus on the micellar properties of dendritic polymers having both hydrophobic and hydrophilic domains.

This chapter consists of four main parts. Section 2.2 is an overview of dendritic polymers covering the synthesis, structure, and properties of dendritic polymers. A discussion on the various strategies used to synthesize amphiphilic dendritic structures follows in Section 2.3. The self-assembly of dendritic amphiphilic polymers at the air-water interface is considered in Section 2.4, with emphasis on the conformational changes involved in building these assemblies and the different topologies observed. The application of these molecules in solubilization, encapsulation, and release is presented in the last section. The various types of host-guest interactions which may be involved, and the influence of micelle and probe structure on these processes are included.

\subsection{Dendritic Polymers}

Dendritic polymers are highly branched macromolecules characterized by a tree-like architecture, incorporating multiple branching levels. This new class of materials has a 
unique combination of features including a compact globular topology with diameters ranging from $1 \mathrm{~nm}$ to over $100 \mathrm{~nm}$, the presence of internal cavities, and a large number of functional groups at the periphery. The broad spectrum of applications being developed for these macromolecules in areas such as microencapsulation, drug delivery, light harvesting, molecular recognition, and catalysis is a result of these remarkable features.

Dendritic macromolecules can be subdivided into dendrimers, dendrigraft polymers, and hyperbranched polymers (Figure 2.1). The classification is based on the degree of structural perfection of the polymers, dendrimers having the most perfect structure while hyperbranched polymers incorporate the largest number of defects. The different degrees of structural perfection attained in each group and the fact that they are obtained by diverse synthetic methods lead to some unique properties and applications for each branched polymer family. A concise comparison of the structure and properties of dendritic polymers and their linear analogues can be found in the literature. ${ }^{5}$

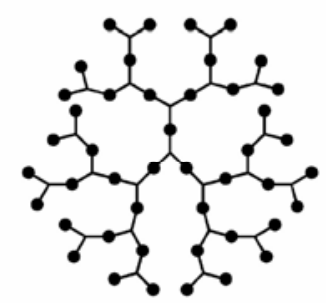

(a)

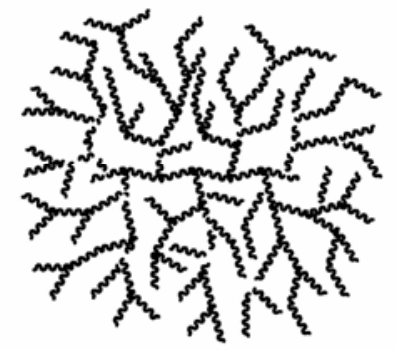

(b)

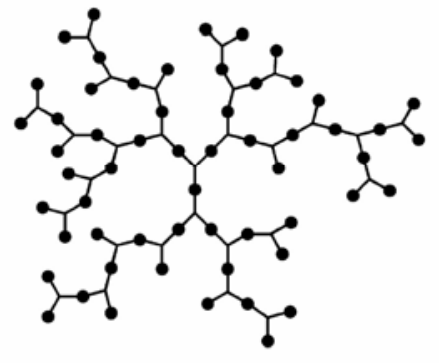

(c)

Figure 2.1. Structure of dendritic polymers: (a) dendrimer, (b) dendrigraft polymer, (c) hyperbranched polymer. 
2.2.1 Dendrimers. Dendrimers are the most structurally perfect group of dendritic macromolecules. They are typically highly symmetrical, essentially monodispersed in size, approaching a globular shape after a certain degree of polymerization, with bonds emanating from a central core, and a large number of chain ends. Dendrimers are mostly synthesized by polycondensation of $\mathbf{A B}_{\mathbf{n}}$ monomers using cycles of protection, condensation, and deprotection reactions. This approach provides full control over the molecular weight and structure of the molecules, unless incomplete or side reactions become significant. These materials can be obtained either from so-called divergent or convergent synthetic schemes. In a divergent or core-first procedure, monomer addition starts from the core and proceeds towards the surface of the molecules. In a convergent procedure, in contrast, dendritic coneshaped fragments (dendrons) are first synthesized starting from the chain ends on the 'outside' using successive coupling reactions at a single reactive 'focal' site. A few dendrons are then assembled into a globular dendrimer in a final coupling reaction. The core of dendrimers is usually referred to as generation $0(G 0)$, while each branched layer produced as a result of polycondensation reactions gives rise to subsequent generations.

The internal structure of dendrimers was first modeled by de Gennes and Hervet, ${ }^{6}$ who proposed a segmental density profile with a minimum at the center of the molecules and increasing towards the outside. While this model has been able to account for the presence of internal voids in dendrimers, ${ }^{7,8}$ others have demonstrated experimentally and through modeling that many of these macromolecules actually have a density maximum at the center. $^{9-12}$ A more recent modeling study on nonideal dendrimers using Monte Carlo simulations has reconciled the two viewpoints, by predicting a density profile with a 
maximum at the core for low $(G<5)$ generation dendrimers, but a maximum density at the shell for higher generations $(G \geq 5) .^{13}$

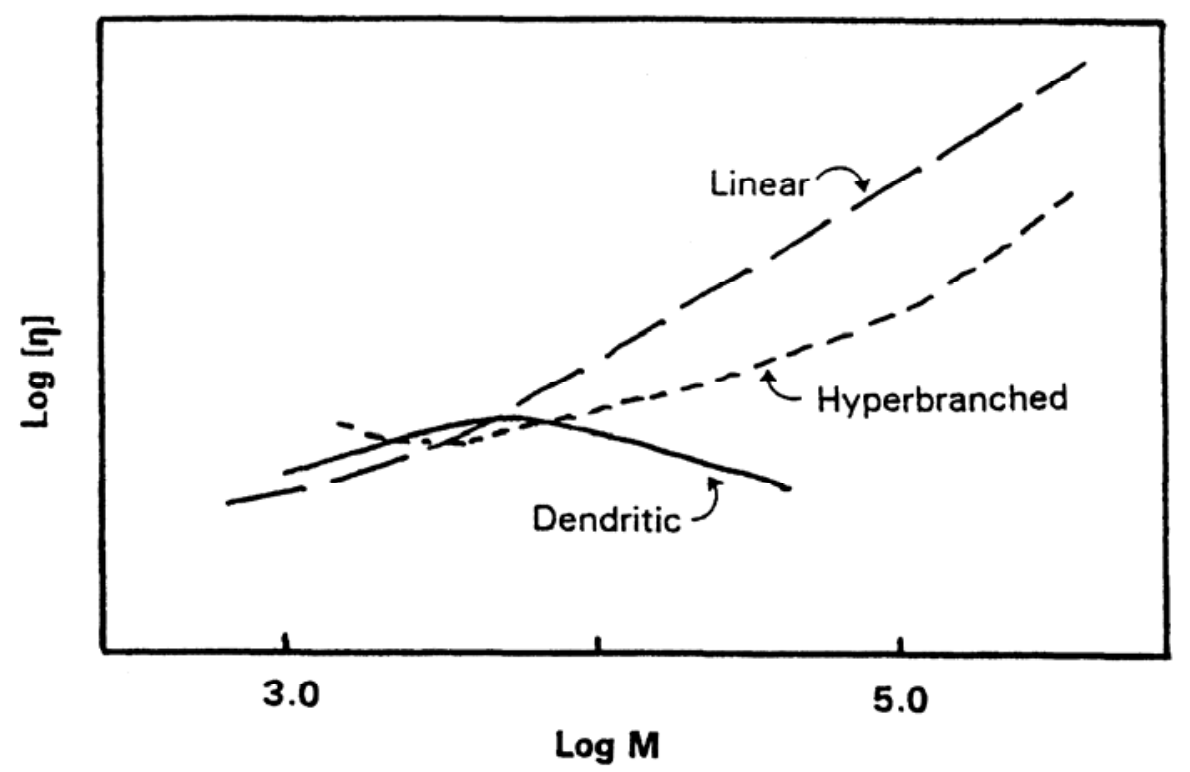

Figure 2.2. Dependence of intrinsic viscosity on molecular weight for linear polymers, hyperbranched polymers, and dendrimers. ${ }^{16}$

The intrinsic viscosity of dendrimers displays a characteristic maximum at molecular weights corresponding to intermediate generation numbers (G4-G7, depending on composition), which is absent in linear and hyperbranched polymers (Figure 2.2). ${ }^{14-17}$ This trend can be represented alternately by a minimum in hydrodynamic density, since according to the Einstein equation the intrinsic viscosity $[\eta]=2.5 \mathrm{~N}_{\mathrm{A}} \mathrm{V}_{\mathrm{h}} / \mathrm{M}$ is inversely proportional to the hydrodynamic density $\mathrm{M} / \mathrm{V}_{\mathrm{h}}$. The hydrodynamic density minimum is due to the fact that while the molecular mass of dendrimers increases exponentially for successive generations, the molecular volume only increases with the third power of the generation number. This was 
shown to induce a change in shape of the dendrimer molecules from ellipsoidal at low generations to a perfect sphere at higher generations. ${ }^{18}$ Deviations from the Mark-HouwinkSakurada equation $[\eta]=K M^{\text {a }}$ were also reported for high molecular weight dendrimers, in contrast to linear and other branched polymers.

Dendritic-linear hybrid polymers which are 'crossbreed' structures combining a dendrimer and a linear polymer have also been synthesized. The synthesis is accomplished either by the growth of a linear polymer chain from the focal point of a dendritic molecule (usually a dendron), or by coupling a dendron with a preformed linear chain. ${ }^{19}$ In such a situation, the properties of the hybrid molecules depend not only on the proportions of the linear and dendritic components, but also on their chemical functionality and the spatial arrangement of the components within the hybrid molecules.

2.2.2 Dendrigraft Polymers. These highly branched molecules are synthesized from polymeric chains, typically assembled according to a generation-based scheme analogous to dendrimers, that relies on cycles of substrate functionalization and grafting. In contrast to dendrimers, the coupling sites are distributed randomly on the substrate rather than strictly located at the chain ends, however. ${ }^{4}$ The reaction of a functionalized linear substrate with preformed chains yields a generation 0 or $G 0$ polymer. Subsequent functionalization and grafting steps lead to higher generation $(G 1, G 2 \ldots)$ dendrigraft polymers. Dendritic macromolecules with a high branching functionality $\left(f_{\mathrm{w}}=10-10000\right)$, a high molecular weight $\left(M_{\mathrm{w}}=10^{3}-10^{8}\right)$, and a narrow size distribution $\left(M_{\mathrm{w}} / M_{\mathrm{n}} \leq 1.1\right)$ are obtained after only 1-4 reaction cycles. These highly branched molecules are synthesized from polymeric chains, typically assembled according to a generation-based scheme analogous to dendrimers, that relies on cycles of substrate functionalization and grafting. 
Various techniques have been used to establish structure-property relations for arborescent polystyrenes. ${ }^{20-23}$ The values of the exponent $v$ observed in scaling relations $Y \sim M_{w}^{v}$ (values expected for hard spheres given between parentheses) are $v=0.2(0.33)$ for the radius of gyration, $v=-0.9(-1)$ for the second virial coefficient, and $v=-0.34(-0.33)$ for the translational diffusion coefficient, in agreement with a rigid sphere morphology. ${ }^{27}$ While scaling relations in dilute solutions suggested a rigid sphere morphology, fluorescence quenching measurements ${ }^{21}$ demonstrated that part of the molecule remains essentially inaccessible to large (polymeric) quencher species. Small-angle neutron scattering (SANS) measurements ${ }^{22}$ have been used to probe the segmental density of arborescent polymer molecules. Modeling of the scattering profiles yielded a dense core of uniform density, surrounded by an outer layer of more diffuse chains. The fluorescence and SANS results are consistent with a core-shell morphology, whereby the outer chains grafted in the last generation form a soft shell around the core stiffened by a high segmental density and multiple branching sites.

Arborescent polystyrenes were found to exhibit a maximum in intrinsic viscosity as a function of molecular weight in analogy to dendrimers (Figure 2.3), ${ }^{23}$ albeit the maximum was less pronounced and was observed at higher molecular weights. It is possible to fine-tune the physical properties of these polymers by varying structural parameters such as the branching functionality $\left(f_{\mathrm{w}}\right)$ of the substrate or the size $\left(M_{\mathrm{w}}\right)$ of the side chains. For example, it was shown that the hydrodynamic radius $\left(R_{\mathrm{H}}\right)$ of arborescent polystyrenes with short side chains $\left(M_{\mathrm{w}}=5000\right)$ remained almost constant in cyclohexane (poor solvent) and in toluene (good solvent), while it increased significantly for polymers with long side chains $\left(M_{\mathrm{w}}=\right.$ $30000) .{ }^{30}$ 


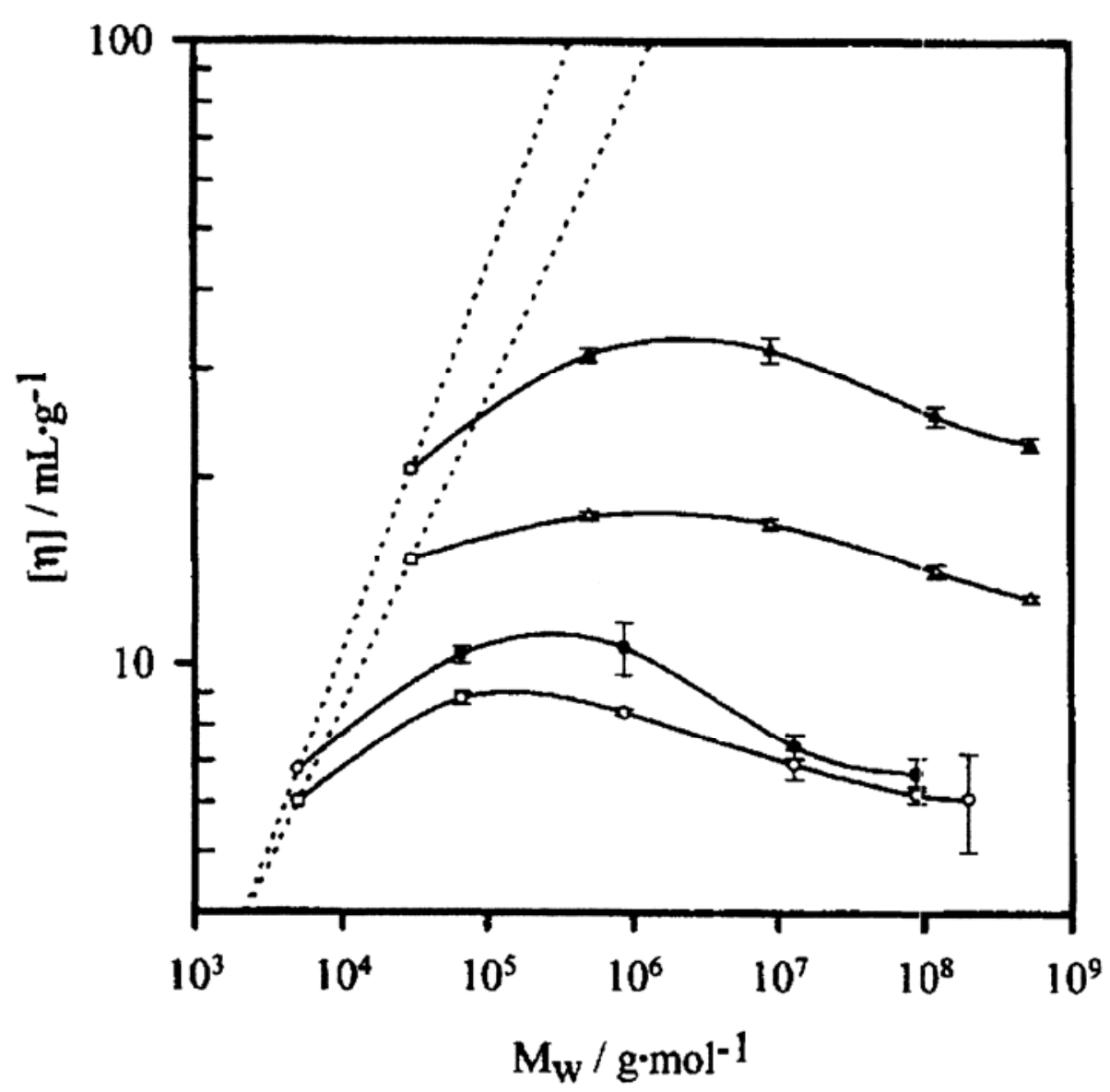

Figure 2.3. Dependence of intrinsic viscosity on molecular weight for arborescent polystyrenes of different structures, from top to bottom: side chain $M_{\mathrm{w}} \approx 30000$ in toluene at $25{ }^{\circ} \mathrm{C}$, in cyclohexane at $34.5^{\circ} \mathrm{C}$; side chain $M_{\mathrm{w}} \approx 5000$ in toluene at $25{ }^{\circ} \mathrm{C}$, in cyclohexane at $34.5^{\circ} \mathrm{C}$. The dotted lines are for linear polystyrene. ${ }^{23}$

2.2.3 Hyperbranched Polymers. Hyperbranched (or statistically branched) polymers are typically synthesized by polycondensation reactions of $\mathbf{A B}_{\mathbf{n}}$ monomers without selective protection, as originally described by Flory. ${ }^{24}$ The synthesis is often achieved through a onepot reaction, making it difficult to control the size (molecular weight) distribution of the 
molecules. ${ }^{25-27}$ Because of the poorly defined structure of hyperbranched polymers, the concept of generation number is not as clear as for the other dendritic polymer families. Hyperbranched polymers are usually characterized in terms of molecular weight and degree of branching (DB), defined as the fraction of repeat units forming branching points in the molecule ( $\mathrm{DB}=1$ for a perfect dendrimer). The establishment of structure-property relations for hyperbranched polymers has remained a challenge, due to the fact that their properties depend on the structure of the repeat units, the number of structural defects present, as well as the nature of the end groups. ${ }^{28}$ Few studies aimed at elucidating structure-property relations for hyperbranched polymers have been based on comparisons with suitable analogues, since this is strictly possible only when the branched molecules and the reference compounds have the same kind and number of functional groups. ${ }^{28}$ In a comparison of linear, dendritic, and hyperbranched polyesters, ${ }^{29}$ for example, it was found that when the number of functional groups present in each molecule remains constant, the glass transition temperature $\left(T_{\mathrm{g}}\right)$ is actually independent of the architecture of the molecules, but is influenced by the type of functional groups present. Nonetheless, branched polyesters were found to have a higher solubility than their linear analogues. Hyperbranched polyphenylenes were likewise found to be soluble in common organic solvents while their linear counterparts were insoluble. ${ }^{30}$ These polymers also exhibit a very low intrinsic viscosity due to their highly branched architecture, in analogy to hyperbranched molecules in general, ${ }^{31-33}$ albeit a roughly exponential increase in $[\eta]$ with molecular weight is observed (Figure 2.2).

Small-angle x-ray scattering (SAXS) measurements have been used to compare the morphology of hyperbranched polyols of generations $G 2-G 5$ to that of polyamidoamine (PAMAM) dendrimers of generations $G 1-G 4 .{ }^{34}$ Due to the small size of the molecules, a 
sufficiently wide scattering vector $(q)$ range was unfortunately not available for the accurate determination of particle shape factors and segment density profiles. Nonetheless, the results obtained for dendrimers were found to be consistent with monodispersed species with a uniform sphere-like segment density distribution, while the scattering behavior of hyperbranched polymer solutions was characteristic of species with a wide distribution of molecular sizes, shapes and weights, and an inhomogeneous segment density distribution.

SAXS and dynamic light scattering (DLS) measurements have also been used to study dilute solutions of hyperbranched polystyrenes in toluene. ${ }^{35}$ The radius of gyration/hydrodynamic radius ratio $\left(R_{\mathrm{G}} / R_{\mathrm{H}}\right)$ can serve to characterize the segment density profile of microgels and branched polymers in solution. ${ }^{36-38}$ It is expected to equal 0.775 for hard spheres with a uniform segmental density distribution, while values ranging from 1.231.46 have been reported for linear polystyrene in toluene. The values obtained for hyperbranched polystyrenes were in the range $0.82-0.89$, and therefore consistent with a hard sphere-like morphology.

\subsection{Amphiphilic Dendritic Polymers}

While some dendritic polymers like polyamidoamide (PAMAM) and polypropylenimine dendrimers are able to encapsulate or solubilize guest molecules without further modification, dendritic micelles are generally obtained by chemical modification of dendrimer, dendrigraft, or hyperbranched substrates. Amphiphilic dendrimers are expected to have a relatively sharp core-shell interface, because the structure of the core is uniform and the functional groups making them amphiphilic are present only at the periphery of the molecules (neglecting chain end backfolding inside the core). In contrast, because the 
coupling sites are randomly distributed on substrates serving in the preparation of arborescent copolymers, the interfacial (palisade) region of mixed composition between the hydrophobic core and the hydrophilic shell of the molecules should be significant. Hyperbranched amphiphilic polymers, on the other hand, may not even display a core-shell morphology due to the poorly defined architecture of the molecules. Two main techniques have been used to impart micellar properties to dendritic cores: chain-end modification, and coupling of a dendritic substrate with polymer chains. The synthesis of dendritic-linear hybrids, while conceptually related to the method based on coupling with linear polymer chains, is presented separately. Below are a few examples that demonstrate the use of these techniques.

2.3.1 Functionalization of Chain Ends. Because of their perfect structure and the large number of chain ends present, it is convenient to functionalize dendrimers at their periphery. Newkome et al. ${ }^{39}$ and Hawker et al. ${ }^{8}$ first synthesized dendrimer micelles by functionalizing hydrophobic cores derived from hexatricontaalchohol and poly(aryl ether)s, respectively, with polar carboxylic acid groups. Other polar groups such as hydroxyl functionalities have also been introduced on dendrimer cores by various methods. ${ }^{40-43}$

Functionalization of the chain termini of hyperbranched polymers is not a popular method for the synthesis of amphiphilic dendritic polymers, because these molecules show no clear differentiation between the interior and the periphery due to their poorly defined structure. Nonetheless, Haag et al. have been able to take advantage of the chemically distinguishable linear and terminal glycerol units of hyperbranched polyglycerols to generate a core-shell architecture: ${ }^{44}$ Selective conversion of the terminal 1,2-diol units to the corresponding acetals or ketals yielded hyperbranched polymers behaving like inverse micelles. 
2.3.2 Coupling of Dendritic Substrate with Polymer Chains. The addition of hydrophobic or hydrophilic polymer segments on dendritic substrates is another method developed to obtain dendritic micelles. Two approaches have been used to achieve this, namely the 'grafting onto', and the 'grafting from' techniques. The 'grafting onto' scheme relies on the introduction of reactive sites on the substrate, followed by coupling with polymer chains of a different composition. In the 'grafting from' method the dendritic core is first functionalized with dormant initiating sites, which are subsequently activated and used to initiate the polymerization of a monomer.

Liu et al. $^{45}$ have obtained unimolecular micelles by grafting amino-terminated poly(ethylene glycol) (PEG) chains onto a hydrophobic dendrimer core, synthesized by coupling various mucic acid derivatives and 1,1,1-tris(4'-hydroxyphenyl)ethane using dicyclohexylcarbodiimide (DCC) and dimethylaminopyridine (DMAP) as promoters. The DCC/DMAP promoter combination also served in the final coupling reaction of the aminoterminated PEG and the core. While the 'grafting onto' methodology has not received much attention for the synthesis of dendrimer micelles, the 'grafting from' method has been extensively applied, in particular for dendrimer-like star copolymers of low branching functionality, by using dendrimer derivatives as macroinitiators. ${ }^{46,47}$

The 'grafting onto' approach has been likewise applied to the synthesis of arborescent amphiphilic copolymers. For example, 'living' anionic poly(2-vinylpyridine) chains were coupled with chloromethylated or acetylated polystyrene arborescent substrates of different generations to produce copolymers with poly(2-vinylpyridine) segments. ${ }^{48,49}$ Copolymers based on polystyrene cores of generations $G 0$ (overall copolymer generation $G 1$ ) and above, when dissolved in dilute aqueous $\mathrm{HCl}$ solutions, were shown to exist as unimolecular (non- 
associated) species. The 'grafting from' technique also served in the synthesis of arborescent polystyrene-graft-poly(ethylene oxide) copolymers, whereby poly(ethylene oxide) chains were grown from alcoholate groups attached at the chain termini of the arborescent polystyrene substrates. ${ }^{50} \mathrm{~A}$ similar technique was employed by Taton et al. ${ }^{51}$ to synthesize a dendritic graft copolymer by anionic growth of 12 outer poly(ethylene oxide) chains from a hexaarm polystyrene star core. Inverse dendrigraft micelles have also been derived ${ }^{52}$ from triand tetraarm star-branched poly(ethylene oxide) substrates. The terminal - $\mathrm{OH}$ groups of the star polymers were derivatized with a branching agent to double the number of hydroxyl sites, reacted with 2-bromopropionyl bromide, and used to polymerize styrene by atom transfer radical polymerization (ATRP) with $\mathrm{CuBr} / 2,2^{\prime}$-bipyridine as catalyst.

The synthesis of dendritic micelles from hyperbranched cores has been achieved according to a 'grafting onto' scheme, by coupling allyl-terminated poly(ethylene oxide) segments with hyperbranched polysiloxysilanes via hydrosilylation. ${ }^{53}$ The 'grafting from' technique also served to synthesize amphiphilic hyperbranched copolymers, for example using a hyperbranched polyester substrate derived from 4,4-bis(4'-hydroxyphenyl)valeric acid, by esterification with 3-(chloromethyl)benzoyl chloride to serve as macroinitiator in the cationic ring opening polymerization of 2-methyl-2-oxazoline. ${ }^{54}$

2.3.3 Dendritic-linear Molecules. The amphiphilic dendritic-linear hybrids introduced by Gitsov and Fréchet ${ }^{55,56}$ have opened a new front in the synthesis of surfaceactive agents. The reactions usually involve coupling of preformed dendrons with either linear, tri- or tetraarm polymers to form block copolymers. For example, $G 1$ to $G 4$ dendrons with a bromide functionality at their focal point have been coupled with either poly(ethylene oxide) or poly(ethylene glycol) (PEO or PEG) chains in the presence of sodium hydride. 
Hybrid polymers have also been synthesized according to a divergent scheme whereby one or more dendritic arms were grown from a terminally functionalized linear backbone. Linear polystyrene chains functionalized at one end with a primary amine group were thus used to grow polypropylenimine dendrimers of different generations. ${ }^{57} \mathrm{~A}$ similar approach also served to synthesize diblock and triblock copolymers by growing carbosilane dendrons from allyl-terminated PEO chains. ${ }^{58,59}$ The major distinction between amphiphilic dendritic-linear hybrids and other amphiphilic dendritic molecules is that most hybrids behave like surfactants and block copolymers, forming micelles by aggregation once a minimum concentration $(\mathrm{CMC})$ is attained.

\subsection{Behavior of Amphiphilic Dendritic Polymers at the Air-water Interface}

In analogy to block copolymer micelles, amphiphilic dendritic polymers can be referred to as regular micelles with the hydrophobic domain forming the core and the hydrophilic moieties forming the corona, or reverse (inverse) micelles with the hydrophobic domain forming the corona. While regular micelles are soluble in aqueous media, most reverse micelles are soluble only in organic solvents. Depending on the relative size of the core and the corona, each type of micelle can be further divided into star and crew-cut structures. For star micelles the dimensions of the core are small relative to the corona, while crew-cut micelles have relatively large cores. Crew-cut dendritic systems such as surfacemodified dendrimers have the ability to form supramolecular assemblies at interfacial boundaries. A popular method to study the formation of monolayer films at the air-water interface is the Langmuir-Blodgett force balance technique. This not only provides information on the molecular orientation of amphiphiles, phase transitions, and conformation 
changes at the air-water interface, but also has the unique advantage that it affords orientation control on the molecular scale, and hence topology control for the assemblies.

2.4.1 The Langmuir-Blodgett Technique. A typical Langmuir balance consists of a trough on top of which is a movable barrier for compressing and/or decompressing the film at the air-water interface. In a typical experiment, the trough is filled with a subphase (usually Milli-Q water). A small volume of an organic solution of the amphiphiles is placed onto the subphase. The amphiphiles spread over the entire water subphase forming a monolayer (Langmuir monolayer), with their hydrophobic portion in contact with air and their hydrophilic portion in contact with the water. After evaporation of the spreading solvent, a movable barrier is used to constrain progressively the amphiphiles to a smaller area on the water surface, and thus push the molecules closer until they are oriented. The Langmuir monolayer thus formed can be transferred to a solid substrate by lowering and/or raising it through the air-water interface to produce a Langmuir-Blodgett film. ${ }^{60}$ The transfer efficiency of the Langmuir-Blodgett film is denoted by the transfer ratio, which is the ratio of the decrease in monolayer area during film deposition to the area of the substrate. For ideal deposition, the transfer ratio equals 1.

2.4.2 The Langmuir Isotherm. The phase changes a monolayer undergoes as it is compressed on the surface of the subphase may be identified to some extent by monitoring the surface pressure $\pi$ as a function of the area occupied by the film. The surface pressure may be measured by the Wilhelmy plate method that relies on the measurement of a force (due to surface tension) on a plate or other object suspended so that it is partially immersed in the subphase. ${ }^{61}$ The presence of a monolayer film affects the surface tension of the subphase. 
The result is a reduction in surface tension relative to the pure subphase, and the surface pressure $\pi$ is given by

$$
\pi=\gamma_{0}-\gamma
$$

where $\gamma_{0}$ is the surface tension of the pure subphase, and $\gamma$, the surface tension of the monolayer-covered surface. A plot of $\pi$ versus area per molecule is called a Langmuir isotherm.

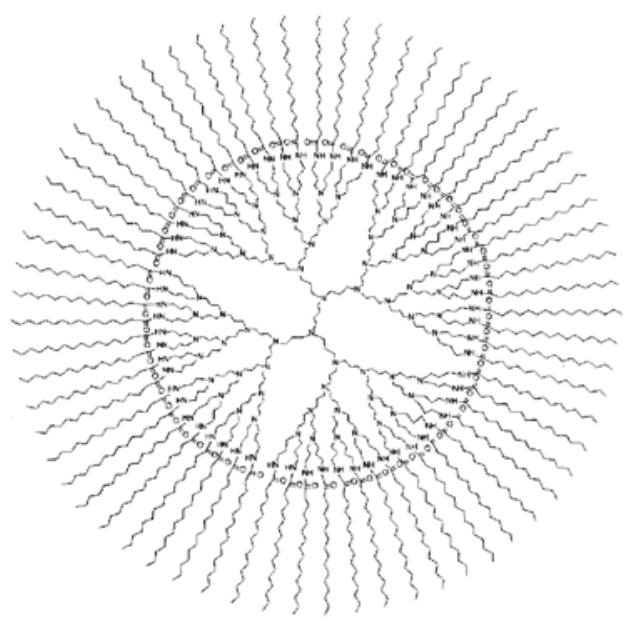

(a)
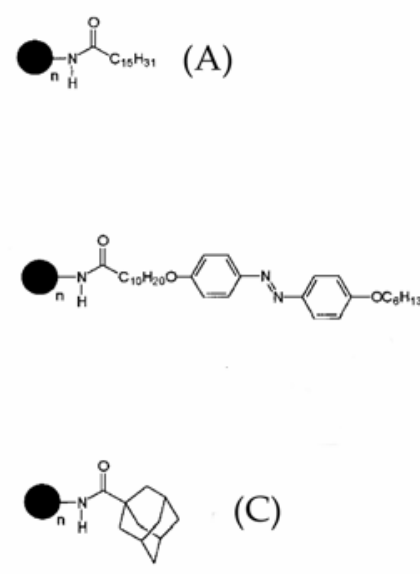

(C)

Figure 2.4. (a) Polypropylenimine dendrimers modified with alkyl chains (A), (b) three types of hydrophobic chains used to modify the surface of the hydrophilic dendrimers. ${ }^{62}$

\subsubsection{Conformation of Dendritic Amphiphilic Polymers at the Air-water}

Interface. Monolayer films of amphiphilic micelles derived from generations G1-G5 polypropylenimine dendrimers have been studied at the air-water interface. ${ }^{62}$ These micelles were synthesized by reacting the peripheral amine groups on the substrates with palmitoyl chloride (A), pentafluorophenyl 11-[4-(4-hexyloxyphenylazo)phenyloxy] undecanoate (B), 
or 1-succinimidoyl adamantane carboxylate $(\mathbf{C})$ as shown on Figure 2.4. Micelles of $\mathbf{A}$ and $\mathbf{B}$ formed stable monolayers, as indicated by the sharp rise in surface pressure observed upon compression (Figure 2.5), while compression isotherms for $\mathbf{C}$ displayed a low surface pressure even at high compression, a behavior typical for unstable monolayers.
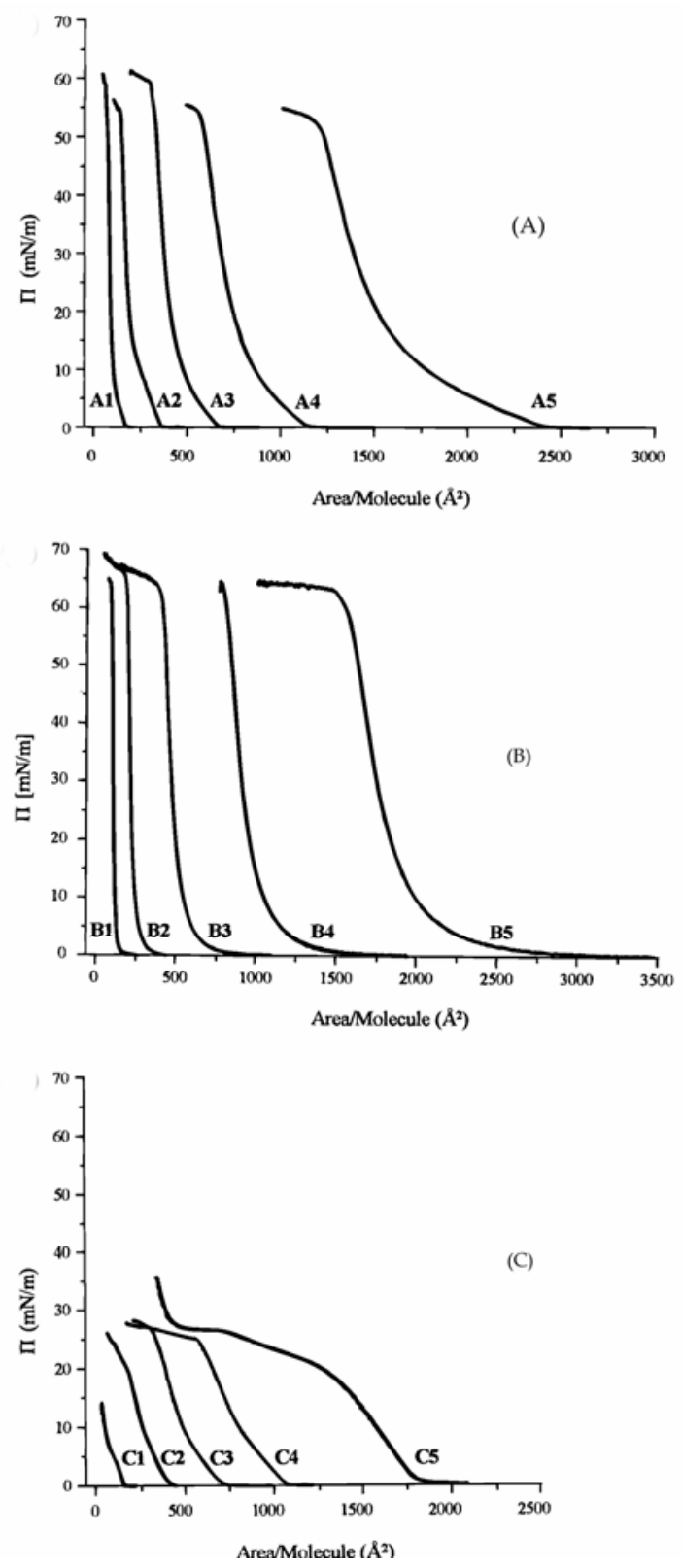

Figure 2.5. Langmuir isotherms for different generations of functionalized polypropylenimine dendrimers at $25^{\circ} \mathrm{C} .{ }^{62}$ 
Decompression isotherms for all monolayers showed irreversibility due to aggregation. This clearly did not simply result from compression, since aggregates could be detected by Brewster angle microscopy even initially. The experimental area per molecule calculated for the various dendritic micelles (by extrapolation of the sharp rise to zero surface pressure) was consistent with theoretical values calculated for both $\mathbf{A}$ and $\mathbf{B}$, and directly proportional to the number of alkyl chains present on the different generations of dendrimer substrates (Table 2.1).

Table 2.1. Experimental and Theoretical Molecular Areas Calculated as a Function of Generation Number for Modified Polypropylenimine Dendrimers. ${ }^{62}$

\begin{tabular}{ccccc}
\hline Dendrimer & \multicolumn{3}{c}{$\mathrm{A}$} \\
generation & Experimental & Theoretical & Experimental & Theoretical \\
& $(\AA ̊)$ & $(\AA)$ & $(\AA ̊)$ & $(\AA)$ \\
& 107 & 100 & 124 & 124 \\
\hline 1 & 214 & 200 & 241 & 248 \\
2 & 442 & 400 & 521 & 496 \\
3 & 814 & 800 & 1000 & 992 \\
5 & 1600 & 1600 & 1947 & 1984 \\
\hline
\end{tabular}


To account for the formation of superstructures and the linear dependence of the area per molecule on the number of alkyl chains attached, it was suggested that dendrimers of all generations adopted a conformation whereby the hydrophilic polypropylenimine core remained in contact with the aqueous phase, while the alkyl chains oriented themselves perpendicularly to the water surface. A flattened conformation for the core would enable the attached chains to contribute to the molecular area, thus explaining the linear increase in molecular area with the number of alkyl chains attached. This kind of conformation is not accessible for derivative $\mathbf{C}$ because of the bulky adamantane moieties. The dendritic core retains its globular shape, and no linear relationship is observed between the area per molecule and the number of side chains attached.

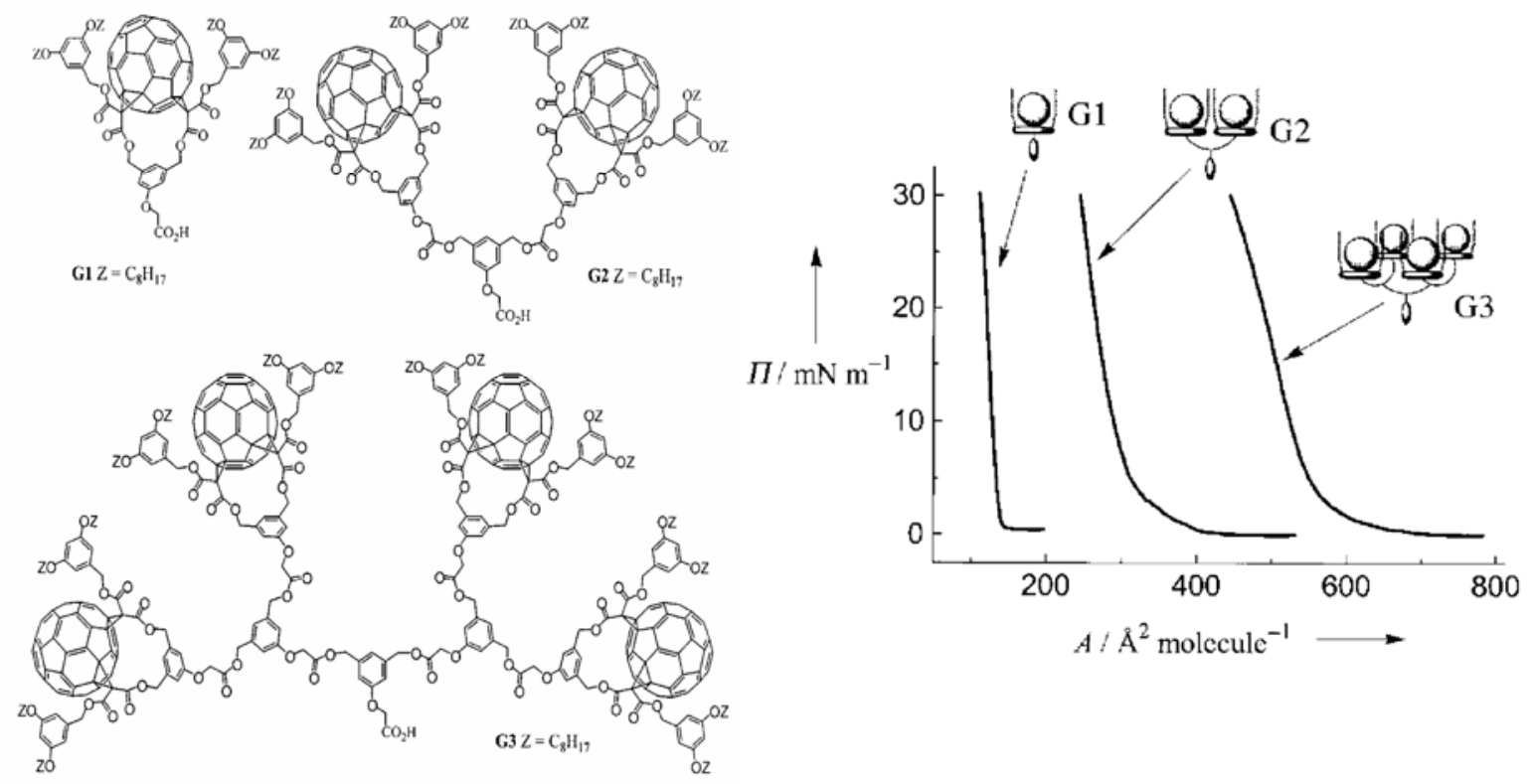

(a)

(b)

Figure 2.6. (a) Amphiphilic dendrimers of different generations bearing fullerene units, (b) Langmuir isotherms of $G 1, G 2$, and $G 3$ amphiphillic dendrimers at $20{ }^{\circ} \mathrm{C} .{ }^{64}$ 
Buckminsterfullerene thin films are being investigated for different applications including microsensors and optoelectronic devices. Unfortunately, bare fullerene aggregates extensively when spread at the air-water interface and yields ill-defined three-dimensional (thick) films rather than monolayers. The attachment of glycodendrons on fullerene spheres was shown to hinder the formation of fullerene aggregates at the air/water interface. ${ }^{63}$ Another method suggested to produce uniform fullerene monolayers ${ }^{64}$ is the attachment of fullerene molecules within amphiphilic dendrons containing octyl chains as outer arms. Compounds of generations $G 1-G 3$ containing 1,2, and 4 fullerene units, respectively, were used in the study (Figure 2.6a). All samples formed stable monolayers at the air/water interface, as indicated by the shape of their respective compression isotherms (Figure 2.6b). The isotherms were fully reversible, without observable hysteresis even after multiple compression-decompression cycles.

The experimental area per molecule values of $140 \pm 7 \AA^{2}, 310 \pm 15 \AA^{2}$, and $560 \pm 30$ $\AA^{2}$ were in good agreement with the dimensions estimated from molecular modeling. The $G 1$ and $G 2$ molecules were found to distribute themselves evenly over the aqueous subphase, but the larger $G 3$ molecules formed unevenly distributed islands. These islands coalesced upon compression to form good quality thin films. Mono- and multilayer Langmuir-Blodgett films of $G 1$ and $G 3$ molecules could be transferred onto silicon or glass substrates hydrophobized with octadecyltrichlorosilane, and produced low-angle Kiessig fringes in grazing incidence $\mathrm{x}$-ray diffraction patterns indicative of a high structural perfection.

Amphiphilic diblock dendrimers containing fullerene units have also been assembled from two dendritic hemispheres, one incorporating four fullerene units within octyl chains on one side and the other one based on a Fréchet-type dendron modified with short 
poly(ethylene glycol) chains (Figure 2.7) ${ }^{65}$ The good hydrophobic/hydrophilic balance and globular architecture of the micelles allowed for the formation of stable Langmuir films. Perfect compression reversibility without hysteresis was also observed in this case, even after four successive compression and expansion cycles, and the good quality of LangmuirBlodgett films transferred on quartz and silicon substrates was confirmed by the observation of low-angle Kiessig fringes in the x-ray diffraction patterns.

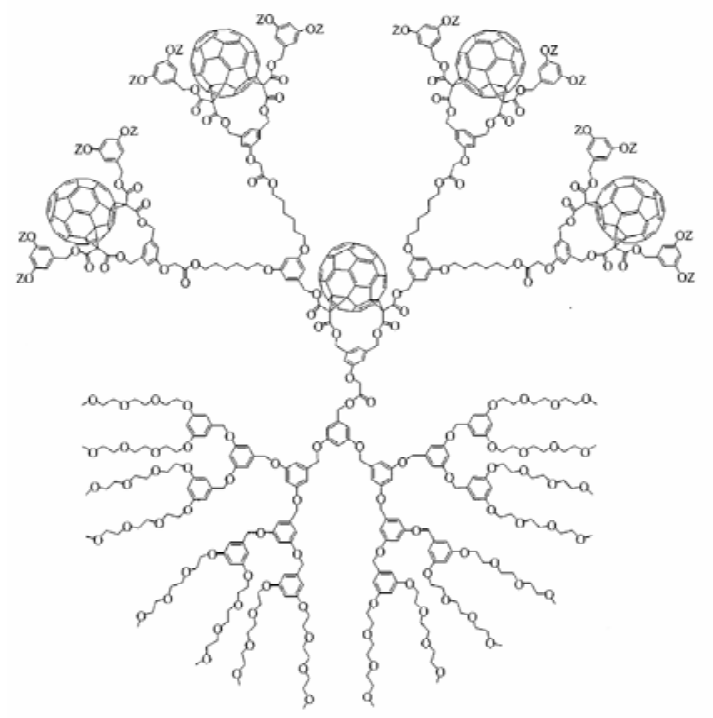

(a)

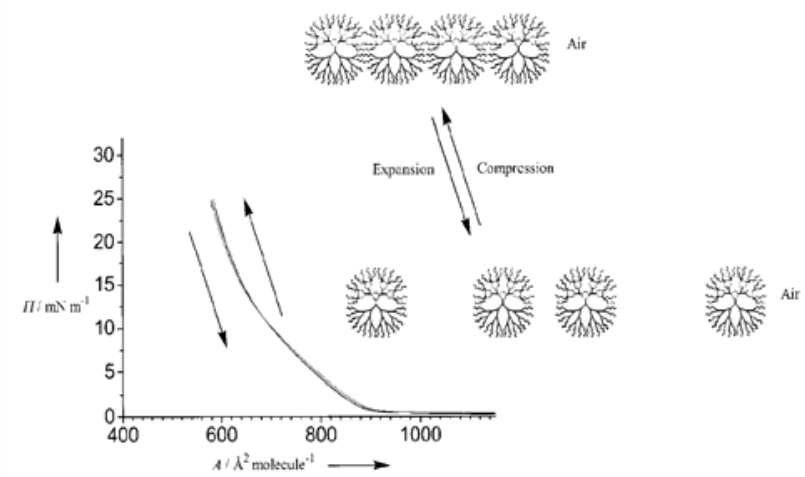

(b)

Figure 2.7. (a) Diblock dendrimer with two hemispherical dendrons, one functionalized with hydrophobic groups $\left(\mathrm{Z}=\mathrm{C}_{16} \mathrm{H}_{33}\right)$ and the other with hydrophilic chains, (b) four successive compression and decompression cycles showing minimal hysteresis. ${ }^{65}$

The conformation of various generations of amphiphilic PAMAM dendrimers derivatized with 12-hydroxydodecanoic acid (HA) was studied by Sui et al. ${ }^{66,67}$ using the 
Langmuir-Blodgett technique. Both Langmuir compression and surface potential-area isotherms showed that these amphiphilic dendrimers were able to form stable monolayers at the air-water interface. All compression isotherms displayed a liquid-expanded phase and a liquid-condensed phase.

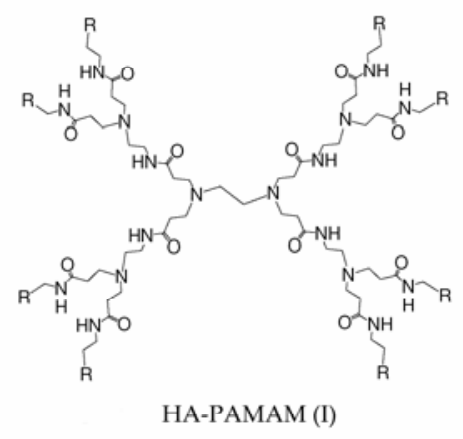

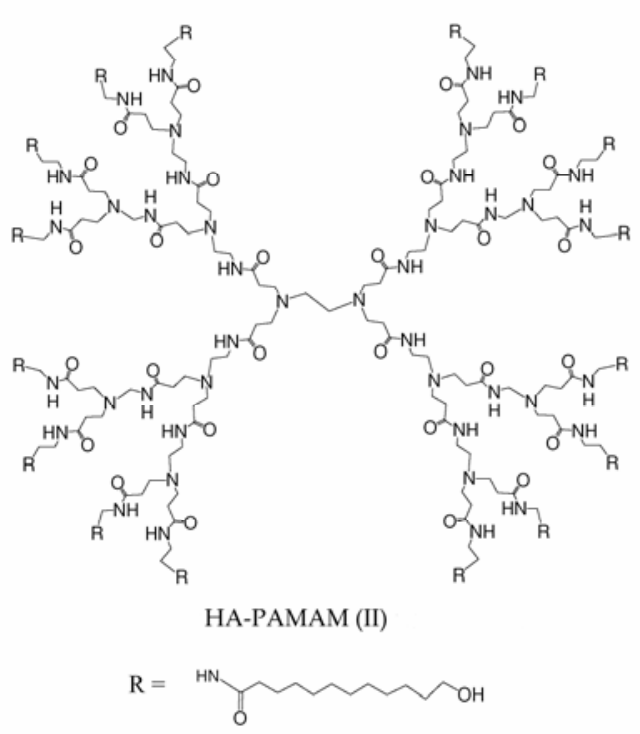

(a)
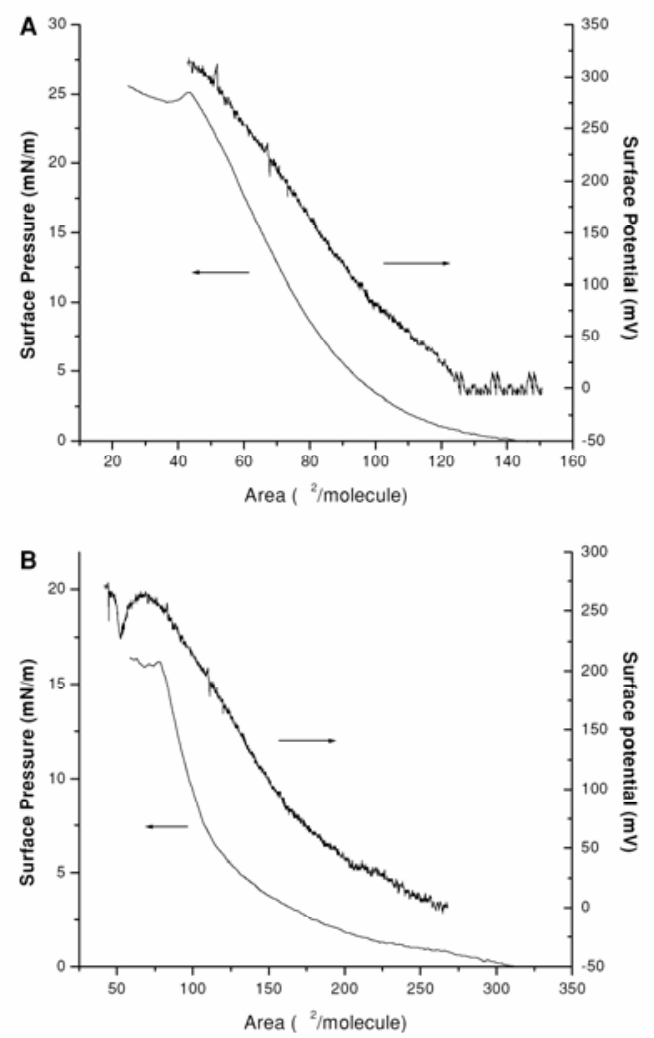

(b)

Figure 2.8. (a) Structure of HA-PAMAM (I) and (II), (b) surface pressure and surface potential isotherms of $\mathbf{I}(\mathbf{A})$; and $\mathbf{I I}(\mathbf{B})$ at air/water interface. ${ }^{66}$

The G1 PAMAM dendrimer I (Figure 2.8), containing 8 HA chains, had a liquid-expanded phase extending from zero surface pressure to $\pi=5 \mathrm{mN} / \mathrm{m}$, and a liquid-condensed phase 
stretching from $5 \mathrm{mN} / \mathrm{m}$ to $25 \mathrm{mN} / \mathrm{m}$. The monolayer film collapsed at surface pressure of $\sim 25 \mathrm{mN} / \mathrm{m}$, while the film formed by the $G 2$ dendrimer II, containing $16 \mathrm{HA}$ chains, collapsed at a lower surface pressure of $\sim 18 \mathrm{mN} / \mathrm{m}$. The results were explained by a random distribution of the molecules on the water subphase, leading to minimal interactions and a low surface pressure at low compression. As the molecules were compressed (decreasing area per molecule), orientation of the alkyl chains led to an increase in surface pressure. This model was supported by surface dipole moments calculated from the surface potential-area isotherms. At low compression, the surface dipole moment is low due to random orientation of the amphiphiles. Compression induces orientation and the surface dipole moment increases. The experimental area per molecule calculated by extrapolation from the liquidcondensed section of the isotherm to zero surface pressure was used to deduce the configuration of the closely packed molecules in the monolayers. Compounds I and II had an area per molecule of 105 and $130 \AA^{2}$, respectively, while a G4 PAMAM core with 64 HA chains had an area per molecule of $160 \AA^{2}{ }^{66}$ Since these values are smaller than the dimensions of the dendrimer core an edge-on configuration was assigned to these molecules, whereby the dendritic micelles are in a more densely packed arrangement than when packed flat on the water surface (face-on configuration). In comparison, micelles based on a PAMAM core similar to II but including 16 peripheral 10,12-pentacosadiyonoic acid units ${ }^{68}$ had an experimental area per molecule of $460 \AA^{2} /$ molecule. Since this is close to the dimensions calculated for the hydrophilic core, a face-on configuration (Figure 2.9a) of distorted PAMAM cores was assigned. The edge-on configuration of I and II was thought to be due to intermolecular hydrogen bonding among the terminal OH groups of HA. 


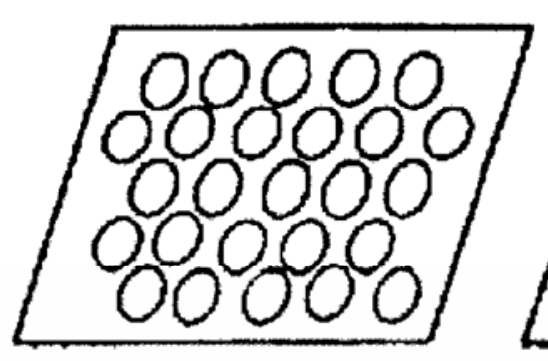

(a)

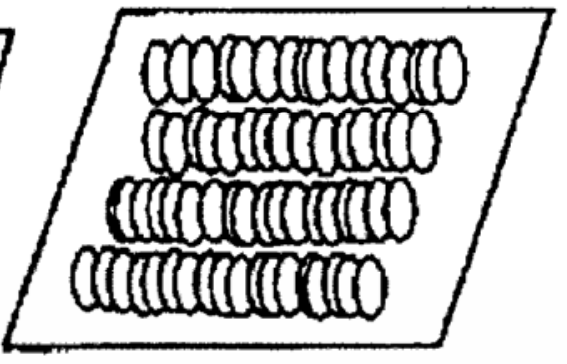

(b)

Figure 2.9. (a) Face-on and (b) edge-on configurations of disk-like molecules in Langmuir and Langmuir-Blodgett films. ${ }^{67}$

While the spreading behavior of amphiphilic systems based on dendrimer substrates is reasonably well understood, fewer studies have been completed using dendrons. One such investigation used hemispherical third-generation PAMAM-type dendrons with a polar ethylenediamine moiety at the focal point and hydrophobic dodecyl chains at the periphery. ${ }^{69}$ The shape of the compression isotherm indicated that the monolayer films produced were stable, and that the molecules existed in a liquid-expanded state. The experimental area per molecule calculated by extrapolation of the liquid-expanded region of the isotherm to zero surface pressure was $780 \AA^{2} /$ molecule. Based on molecular dynamics estimations, the modified PAMAM dendron in a flattened conformation at the air-water interface should occupy an area ranging from $710-960 \AA^{2} /$ molecule. At the other extreme, using the crosssectional area occupied by an alkyl chain in a fatty acid monolayer and assuming hexagonal crystalline packing of the chains, the cross-sectional area of the micelle was estimated to be between $340-480 \AA^{2} /$ molecule. It was therefore suggested that the hemispherical PAMAM 
dendrons adopted an oblate conformation on the water surface, the hydrophobic alkyl chains lying on the hydrophilic core in a distorted conformation, away from the water subphase. The conformation of the alkyl chains was corroborated by electron diffraction results on monolayer films transferred onto silicon wafers, confirming the absence of a crystalline state.

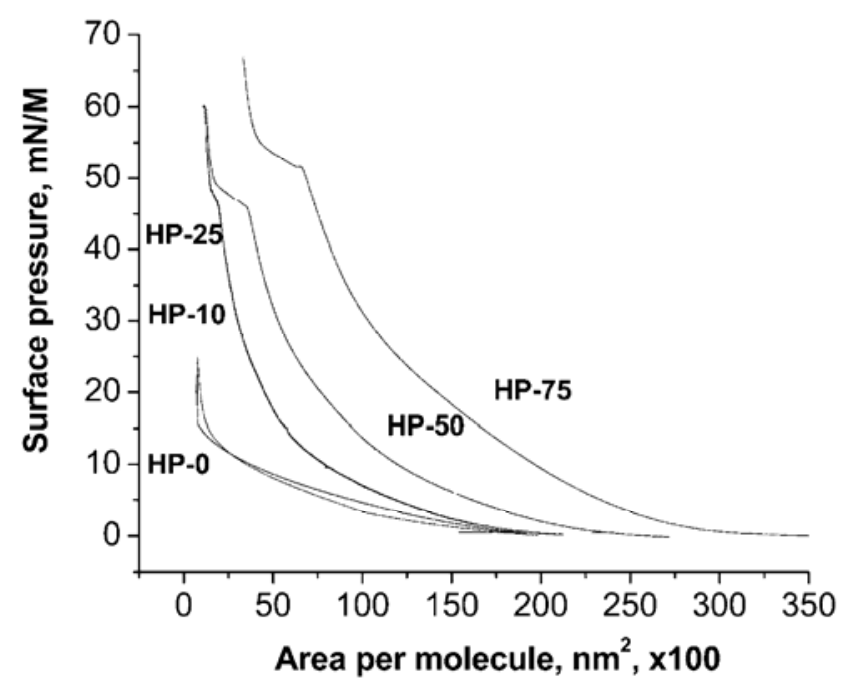

Figure 2.10. Langmuir compression isotherms for HP-0, HP-10, HP-25, HP-50, and HP-75 at the air/water interface. ${ }^{70}$

Hyperbranched amphiphilic micelles have been obtained ${ }^{70}$ by chemical modification of a polyester core containing 12 hydroxyl groups (degree of branching $c a$. 50\%) with stearoyl chloride. To study the influence of composition on the spreading behavior, 5 different compounds were synthesized, HP-0, HP-10, HP-25, HP-50, and HP-75, with respectively $0,10,25,50$, and $75 \%$ of the hydroxyl units substituted. The stability of monolayer films based on these micelles was found to depend markedly on their substitution level. Monolayers prepared from the unsubstituted core and HP-10 were very unstable and 
gradually dissolved with compression, as indicated by the shape of their respective compression isotherms (Figure 2.10). Samples HP-25, HP-50, and HP-75, in contrast, formed stable monolayers with surface pressures at collapse between $60-70 \mathrm{mN} / \mathrm{m}$. Only an estimate for the experimental area per molecule of the micelles could be provided, as their absolute molecular weight was unknown. A good correlation was nonetheless found between the values estimated from the substitution level and the molecular surface area, which increased linearly with the degree of alkyl chain substitution.

Table 2.2. Comparison of Idealized and Estimated Number of Alkyl Chains per Molecule for Hyperbranched Polyesters with Alkyl Substitutents. ${ }^{70}$

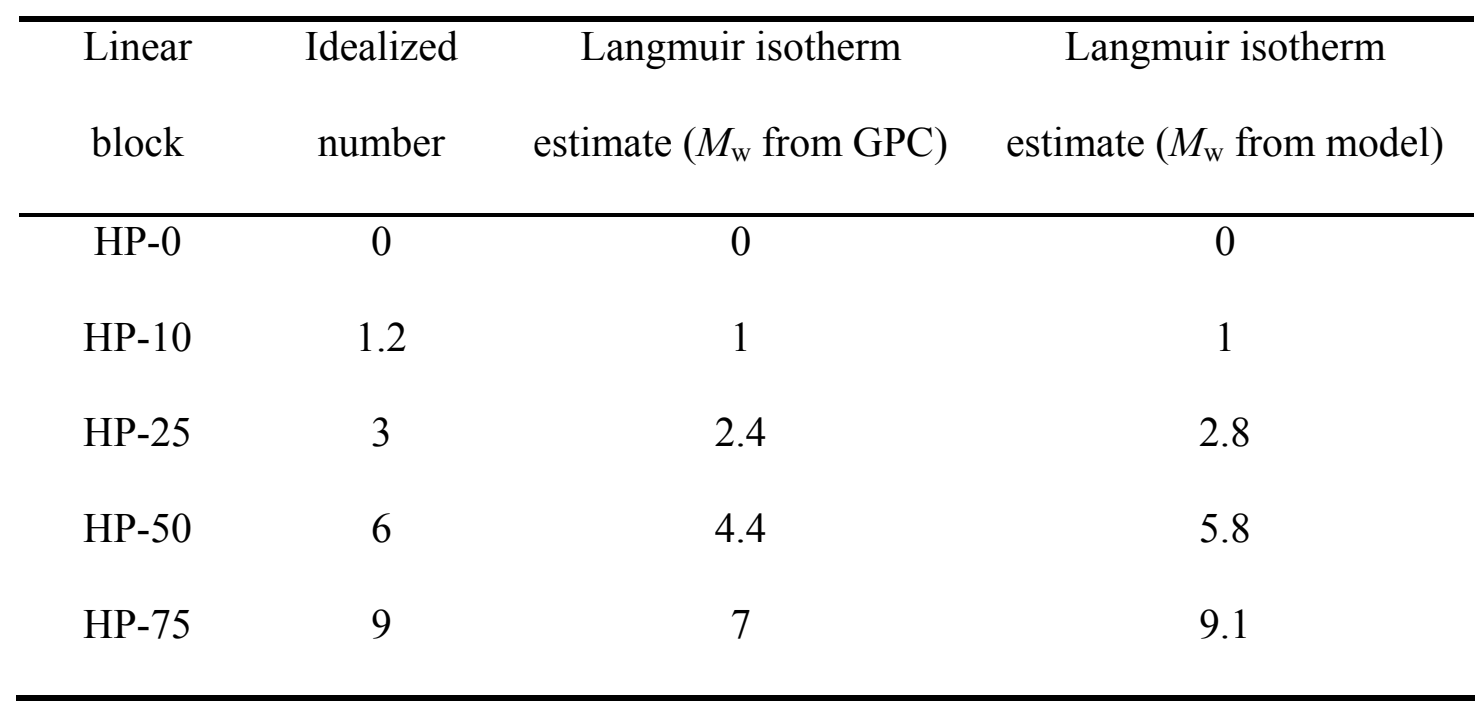

Using the surface area of an alkyl unit in the condensed state of a monolayer, it was possible to estimate the number of alkyl chains per hyperbranched core required for the formation of stable monolayer films (Table 2.2). Thus to achieve a proper hydrophilic/hydrophobic balance for the formation of stable monolayer films and prevent the molecules from dissolving in the water subphase, the weight fraction of the hydrophobic 
phase needed to be at least $30 \%$. The alkyl chains were proposed to adopt an upright orientation on an oblate, flattened core, in analogy to amphiphilic dendrimers. However due to the imperfect structure of the hyperbranched core, the films displayed poor intralayer ordering in x-ray grazing angle incident diffraction measurements.

\subsubsection{Langmuir-Blodgett Film Topology of Amphiphilic Dendritic Polymers by}

Atomic Force Microscopy. Atomic Force Microscopy (AFM) is a powerful imaging technique that has served as a complementary tool to study thin films, and in particular LB films obtained at the air-water interface. AFM measurements also yield information on the structural organization of films produced by adsorption from solution or spin casting. For example the topology of thin films derived from a $G 5$ carbosilane dendrimer coupled with cyanobiphenyl mesogenic units was investigated by Ponomarenko et al. ${ }^{71}$ Films of different thicknesses were obtained by spin casting of dilute polymer solutions on silicon or cleaved mica substrates. Different molecular arrangements of the spherical molecules were observed at the submicrometer level, depending on the concentration of the solution used. Films produced from a $10^{-4} \% \mathrm{w} / \mathrm{w}$ solution were monolayers with single molecules arranged in a rectangular packing motif. Annealing at $130{ }^{\circ} \mathrm{C}$ and slow cooling to room temperature induced a transition to hexagonal packing. Multilayered films obtained from $10^{-3} \% \mathrm{w} / \mathrm{w}$ solutions displayed liquid crystalline behavior with simple smectic orientation. Further increases in film thickness led to aggregated domains of flat-lying smectic layers and edgeon standing smectic layers.

The AFM technique has also been used by Zhai et $\mathrm{al}^{70}$ to obtain information on the topology of Langmuir-Blodgett films of hyperbranched polyester micelles deposited on silicon substrates. Aggregated domains were clearly visible in the film, the size of the 
domains increasing with the degree of alkyl substitution of the hyperbranched cores (Figure 2.11). X-ray reflectivity data obtained in situ at the air-water interface suggested that a significant portion of monolayers deposited at high surface pressure $(35 \mathrm{mN} / \mathrm{m})$ had a bilayer morphology with a hydrophilic layer (dendritic core) in contact with the water phase, and an upper layer of hydrophobic alkyl chains oriented perpendicularly to the subphase. The low height $(0.6-0.8 \mathrm{~nm})$ observed for the aggregate domains by AFM cross-sectional analysis after transfer of the Langmuir film on a silicon substrate was also consistent with a bilayer film morphology similar to that observed in the liquid phase portion of the compression isotherm $(15 \mathrm{mN} / \mathrm{m})$, however with random orientation of the alkyl chains in this case.

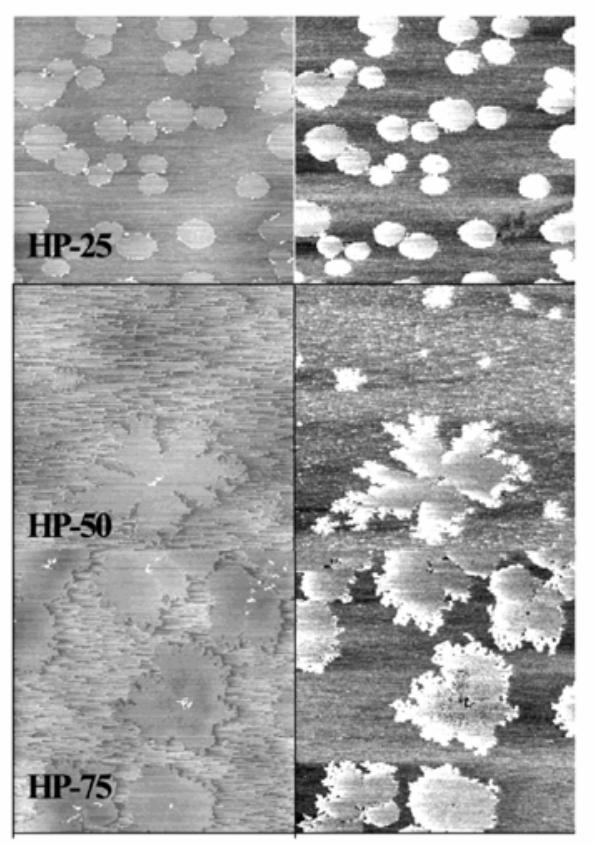

Figure 2.11. Topographical (left) and phase (right) AFM images for Langmuir-Blodgett films of HP-25, HP-50, and HP-75 obtained at a surface pressure of $35 \mathrm{mN} / \mathrm{m}$, scan size $25 \times$ $25 \mu \mathrm{m}^{2} .^{70}$ 


\subsection{Solubilization and Encapsulation}

The fact that unimolecular micelles display no CMC gives them an advantage over surfactant and block copolymer micelles in terms of applications. Their hydrophilic shell makes them soluble in aqueous and other polar media, while the core can solubilize hydrophobic small molecules without fear of changes in micelle morphology or disintegration. The same concept is applicable to inverse unimolecular micelles that are soluble in organic solvents, and capable of solubilizing polar guest molecules in their hydrophilic core. The solubilization process is normally reversible, and the guest molecules are free to diffuse out of the micelles under favorable conditions. For this reason, dendritic unimolecular micelles should be useful for drug delivery, environmental decontamination, catalyst dispersion, and the sustained release of pesticides and fragrances. In dendritic micellar systems the primary function of the shell, be it hydrophilic or hydrophobic, is to enhance the solubility of the micelle host in the dispersion medium, while the guest molecules are encapsulated in the core. Various types of host-guest interactions may be involved in encapsulating the guest molecules within the core, and the solubilization process is greatly affected by the structure of both the solute molecules and the micelles.

2.5.1 Host-guest Interactions. The interactions between the host and guest molecules can be grouped into two main categories: Non-covalent binding, in which the solute physically interacts with the micelle, and covalent binding, in which the solute forms a chemically bonded conjugate. The encapsulation of guest molecules which is based on noncovalent interactions (hydrophobic interactions, physical entrapment, hydrogen bonding, electrostatic bonding, or a combination of these methods) will be discussed in more detail below with the help of examples. 
2.5.1.1 Hydrophobic Interactions. The first method, which is most widely implemented, is based on the nature of the micellar core: Hydrophobic cores interact favorably with hydrophobic solutes. The ability of dendritic micelles to solubilize hydrophobic solutes in aqueous media via hydrophobic interactions was first demonstrated by Newkome et al. ${ }^{72}$ Their unimolecular micelles, incorporating a hydrophobic core derived from hexatricontaalchohol units and outer tetramethylammonium carboxylate functionalities, increased the aqueous solubility of hydrophobic compounds such as diphenylhexatriene and naphthalene. The solubility of naphthalene in $\mathrm{H}_{2} \mathrm{O} / \mathrm{EtOH}$ was thus enhanced $c a$. 4-fold in a $2.3 \times 10^{-4} \mathrm{M}$ micellar solution. Information about the interactions of the hydrophobic probes with the micelles, obtained from fluorescence quenching measurements, optical fluorescence microscopy, and UV spectroscopy, confirmed that the probe molecules were located in the hydrophobic cores. Transmission electron microscopy (TEM) images also showed that the micelles had a uniform size of $30 \pm 5 \AA$.

Unimolecular micelles based on poly(aryl ether) cores with carboxylic acid chain ends $^{8}$ significantly enhanced the aqueous solubility of polycyclic aromatic hydrocarbons such as 2,3,6,7-tetranitrofluorenone (258-fold), pyrene (Py, 120-fold), and anthracene (58-fold) when used at a concentration of $2.13 \times 10^{-4} \mathrm{M}$. A linear relationship between Py solubilization capacity and dendrimer concentration was observed even at concentrations as low as $5 \times 10^{-7} \mathrm{M}$, an indication of the non-existence of a CMC. A significant (4.2-fold) increase in Py solubility to $4 \times 10^{-4} \mathrm{M}$ was observed as $\mathrm{NaCl}$ was added to reach a concentration of $1.5 \mathrm{M}$. This enhancement was attributed to a decrease in the concentration of water (and increased hydrophobicity) of the micellar core as the ionic strength of the aqueous solution was increased. The same group also demonstrated that dendritic micelles 
with a hydrophobic core derived from 4,4-bis(4'-hydroxyphenyl)pentanol and a hydrophilic shell of short poly(ethylene glycol) segments ${ }^{73}$ was able to enhance the aqueous solubility of Py 356-fold at a dendrimer concentration of $1 \times 10^{-4} \mathrm{M}$. The same micelles were used to encapsulate the anti-inflammatory drug indomethacin $(11 \% \mathrm{w} / \mathrm{w})$ in the hydrophobic core and release the molecules in a sustained fashion, demonstrating its potential usefulness in controlled release delivery systems.

The application of pyrene as a polarity-sensitive probe for hydrophobic binding in PAMAM starburst dendrimers end-functionalized with amino groups was explored by Pistolis et al. ${ }^{74}$ The aqueous solubility of Py was increased from $8.0 \times 10^{-7} \mathrm{M}$ to $7.6 \times 10^{-6} \mathrm{M}$ (ca. 10 -fold) for the $G 2$ dendrimers $(\mathrm{MW}=3250$, number of internal voids $=14$ ) at a concentration of $10^{-2} \mathrm{M}$. Evidence for hydrophobic interactions between Py and the core was inferred by steady state fluorescence spectroscopy, based on the relative intensity of the first and third vibronic bands $\left(I_{1}=373 \mathrm{~nm}, I_{3}=383 \mathrm{~nm}\right)$ of Py. The ratio of emission intensities $I_{1} / I_{3}$, sensitive to the microenvironment polarity, decreases from $c a$. $1.6-1.96$ in water to $c a$. 0.6 in $n$-hexane. The $I_{1} / I_{3}$ values for pyrene in $G 0$ and $G 1$ dendrimer micelles were $c a$. $1.5-$ 1.6, close to the values observed in pure water; whereas for $G 2$ dendrimers it was lower ( $c a$. 1.0-1.4). This decrease was attributed to enhanced shielding of the Py molecules from the water molecules for the $G 2$ micelle relative to lower generation dendritic micelles, due to their more globular structure.

Hydrophobic binding by ABA linear-dendritic hybrid copolymers incorporating hydrophobic poly(benzyl ether) dendrons (B) and linear PEG chains (A) was investigated by Gitsov et al. ${ }^{75}$ using UV and steady state fluorescence measurements. A $1.1 \times 10^{-4} \mathrm{M}$ aqueous solution of $G 3$-PEG-G3 triblock copolymer $\left(M_{\mathrm{w}} \mathrm{PEG}=11000\right)$ was found to increase the 
aqueous solubility of pyrene $c a$. 68 -fold to $5.41 \times 10^{-5} \mathrm{M}$. The $I_{1} / I_{3}$ ratio decreased from 1.77 (below the $\mathrm{CMC}$ ) to $1.17-1.37$ above the $\mathrm{CMC}$, indicating that the probe molecules were located in the hydrophobic core of the micelles.

2.5.1.2 Physical Entrapment. Meijer et al. ${ }^{76-78}$ developed the famous 'dendritic box' concept, whereby hydrophobic solutes are physically entrapped within the internal cavities of the micelles. The dendritic box effect is based on the insertion of bulky solute molecules in the internal cavities of a dendritic core, before sealing it with a densely packed shell. The solute becomes physically trapped inside the core and can be released only when the 'dense shell' is disrupted. The dendritic core used to demonstrate this concept was synthesized from a flexible polypropylenimine dendrimer with 64 amino groups at the periphery. Once guest molecules were loaded within the voids, an L-phenylalanine derivative (tertbutyloxycarbonyl-protected $N$-hydroxyl succinimide ester) was used to produce a dense shell (Figure 2.14a). Using ${ }^{13} \mathrm{C}$ NMR spectroscopy, it was demonstrated that the shell was highly hydrogen-bonded and densely packed in solution. A variety of probe molecules of different sizes were entrapped by this method including Bengal Rose, rhodamide, $p$-nitrobenzoic acid, and new coccine. Shape-selective liberation of the guest molecules was also demonstrated: ${ }^{77}$ When two molecules of different sizes were trapped in the core, it was possible to free the smaller ones by partial destruction of the shell. Thus after encapsulating four molecules of Bengal Rose and 8-10 molecules of $p$-nitrobenzoic acid per micelle, it was possible to liberate selectively all the $p$-nitrobenzoic acid molecules upon hydrolysis of the tertbutyloxycarbonyl ( $t$-BOC) groups of the shell with formic acid. The larger Bengal Rose molecules were only liberated after complete hydrolysis of the outer shell with $12 \mathrm{M} \mathrm{HCl}$ under reflux for two hours (Figure 2.12b). 


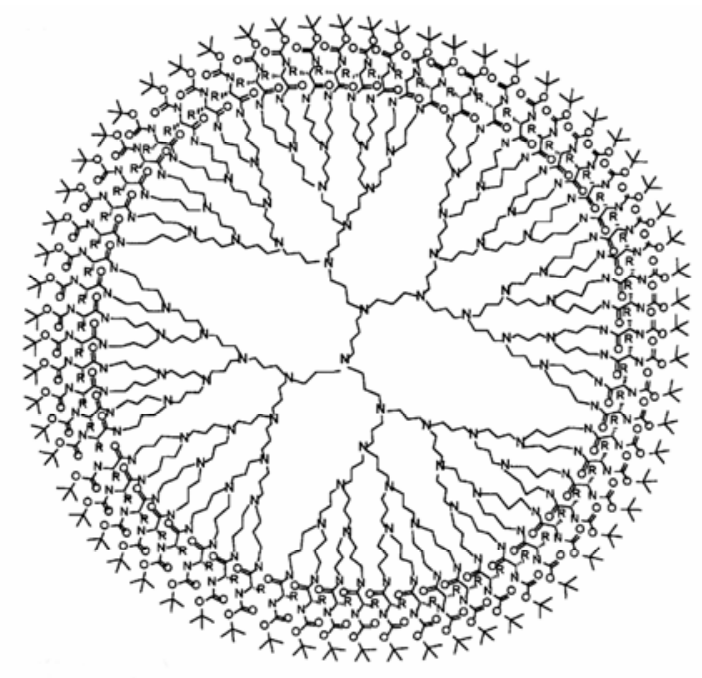

(a)

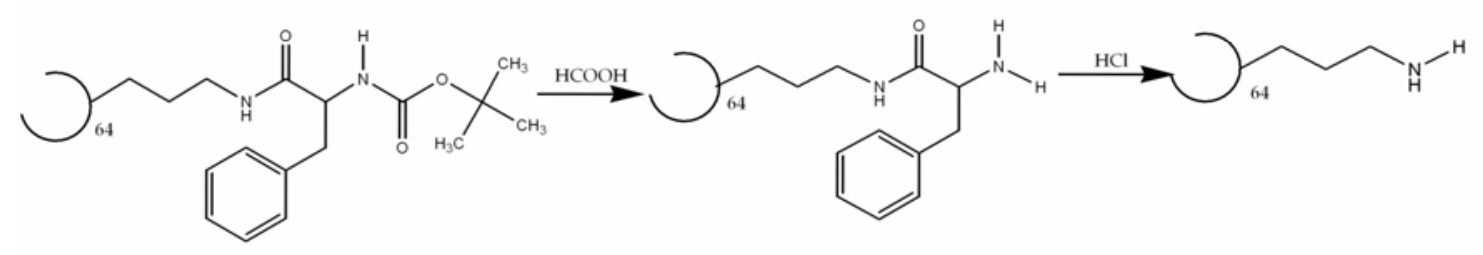

(b)

Figure 2.12. (a) Structure of dendritic box proposed by Meijer ( $R=$ phenyl), (b) cleavage reactions leading to selective liberation of dyes from the dendritic box. ${ }^{77}$

2.5.1.3 Hydrogen Bonding interactions are possible between guest molecules and the core or the surface of dendritic micelles if suitable functional groups are present on both components. Newkome et al. ${ }^{79}$ synthesized a dendritic host with multiple hydrogen-bonding sites based on 2,6-diamidopyridine units in the core, able to bind guest molecules such as glutarimide and barbituric acid. Hydrogen bonding interactions were also shown to dominate for dendritic cores containing naphthyridine units and ionic benzamidinium guest 
molecules. ${ }^{80}$ Based on ${ }^{1} \mathrm{H}$ NMR spectroscopy analysis, the complexes formed were highly stable $\left(K_{\mathrm{a}}=800-1400 \mathrm{M}^{-1}\right)$, the binding constants $K_{\mathrm{a}}$ being independent of the size and chemical nature of the dendrimer. In a subsequent investigation, Santo and Fox ${ }^{81}$ studied the interactions between PAMAM starburst dendrimers and various biological guest molecules including pyridine and trimethadione. The binding constants determined by ${ }^{1} \mathrm{H}$ NMR analysis demonstrated that pyridine could complex with both the internal amido groups and the external amino groups present, while no interactions where observed for trimethadione.

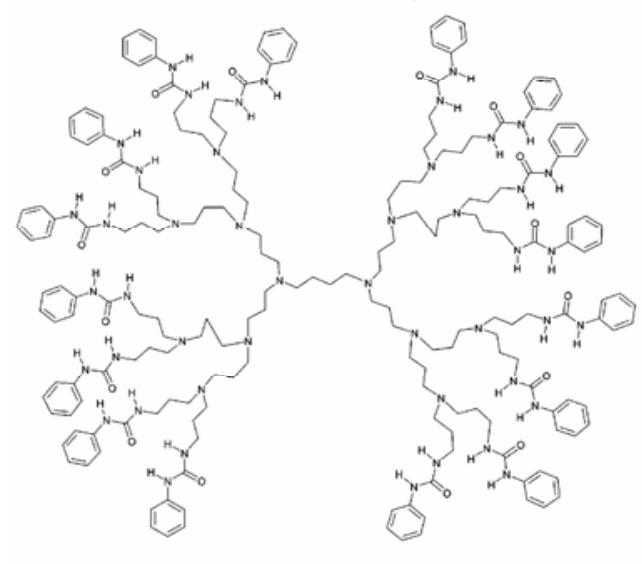

(II)

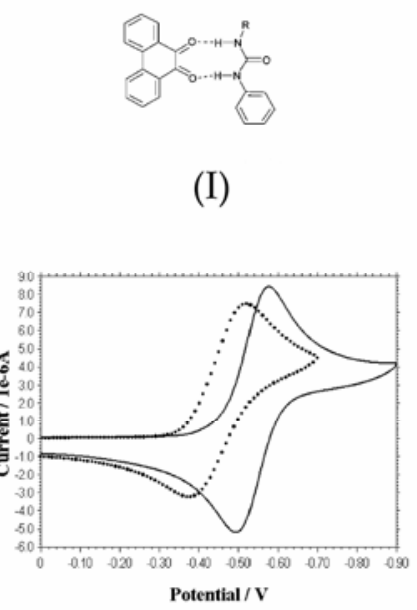

(III)

Figure 2.13. (I) Hydrogen bonding interactions between phenanthrenequinone and terminal phenyl-urea group of dendrimer, (II) phenyl-urea terminated dendrimer, (III) cyclic voltammetry curve for $\sim 9 \times 10^{-4} \mathrm{M}$ phenanthrenequinone in DMF recorded in the absence ($-)$ and presence $(\cdots \cdot)$ of an excess $\left(\sim 6 \times 10^{-3} \mathrm{M}\right)$ of urea-functionalized dendrimer. ${ }^{82}$

${ }^{1} \mathrm{H}$ NMR spectroscopy was also used to show that surface interactions between phenanthrenequinone (I, Figure 2.13) and a phenyl-urea terminated dendrimer (II, Figure 
$2.13)^{82}$ involved hydrogen bonding. The electrochemically controlled complexation of this host-guest system was also investigated using cyclic voltammetry. It was noticed that the addition of an excess of dendrimer $\mathbf{I I}$ to a solution of $\mathbf{I}$ in DMF containing $0.1 \mathrm{M} \mathrm{Bu}_{4} \mathrm{NPF}_{6}$ resulted in a $+90 \mathrm{mV}$ shift in the formal potential (Figure 2.13, III) for the reduction of compound I The potential shift was attributed to increased stability of the radical anionic state of $\mathbf{I}$, due to the binding affinity between the guest molecules and the active groups at the periphery of the dendrimer. The shift in formal reduction potential was accompanied by a decrease in peak current, suggesting that binding to the relatively large dendrimer molecules slowed down the diffusion of guest molecules to the working electrode. This observation was used to further corroborate the occurrence of $\mathrm{H}$-bonding interactions.

Hyperbranched inverse micelles with either polyglycerol or polyethylenimine cores have been used to solubilize polar and anionic guest molecules. ${ }^{83}$ These micelles, which the authors claimed to possess a core-shell morphology in contrast to other hyperbranched micelles, were able to extract polar guest molecules (congo red, methyl red) from aqueous solutions to chloroform. Solubilization of the guest molecules was explained to result from favorable (most likely hydrogen bonding) interactions between the polar solutes and the polar groups present in the hyperbranched cores. For example, a ${ }^{1} \mathrm{H}$ NMR spectrum for methyl red in the polyglycerol micelle showed a significant $(0.2 \mathrm{ppm})$ high field shift for the methyl group signal of methyl red as a result of shielding from the magnetic field by the core-shell structure.

2.5.1.4 Electrostatic Interactions. Baars et al. ${ }^{84}$ studied host-guest interactions in polypropylenimine dendrimers modified with 3,4,5-tris(tetraethyleneoxy)benzoyl units in buffered solutions at $\mathrm{pH} 7$ using two water-soluble anionic dyes, namely 4,5,6,7- 
tetrachlorofluorescein and Rose Bengal. When solutions of the dendritic micelles were titrated against solutions of the two xanthene dyes, a bathochromic shift in the wavelength of the absorption maximum $\left(\lambda_{\max }\right)$ due to interactions between the host molecule and the anionic dyes was observed. For 4,5,6,7-tetrachlorofluorescein, the absorption maximum of the free dye also decreased and a single isosbestic point was observed over the whole titration range, indicating a simple equilibrium between complexed and free dye molecules. The association was attributed to acid-base interactions between the acidic dye molecules and the tertiary amine groups of the dendritic host. This argument was further supported by the observation that the association of the host with 4,5,6,7-tetrachlorofluorescein was more $\mathrm{pH}$-dependent than for the more hydrophobic Rose Bengal dye, for which electrostatic interactions are less dominant.

Amphiphilic PAMAM dendrimer derivatives are also able to bind small hydrophobic acidic molecules, more likely as a result of electrostatic than hydrogen bonding interactions. ${ }^{85}{ }^{1} \mathrm{H}$ NMR spectroscopic analysis was used to monitor the stability of dendrimer complexes with benzoic acid or salicylic acid (as model compounds for acidic drugs) as a function of $\mathrm{pH}$, and led to the conclusion that these acidic probes were able to form stable ion pairs with the internal tertiary amines in the core.

The use of inverse dendritic micelles to solubilize polar guest molecules through electostatic interactions has been likewise documented. ${ }^{86,87}$ Baars et al. $^{86}$ used micelles incorporating a hydrophilic polypropylenimine dendrimer core and hydrophobic linear alkyl chains to extract anionic dyes such as fluorescein, 4,5,6,7-tetrachlorofluorescein, and erythrosine from water into organic solvents (dichloromethane and toluene). The extraction 
efficiency was found to be strongly $\mathrm{pH}$-dependent, decreasing at $\mathrm{pH}>7$ due to less efficient acid-base interactions between the dye and the dendrimer core.

2.5.2 Influence of Micelle and Probe Structure on Encapsulation. The solubilization or encapsulation of probes within dendritic micelles and their subsequent release is a phenomenon that depends on both the architecture of the host and the size and shape of the guest molecules. For example if the guest molecules are encapsulated within the voids of the core, the encapsulation of a larger number of guest molecules should require building a molecule with more voids, i.e. a higher generation dendrimer. Dendritic micelles should have a greater affinity for guest molecules of comparable hydrophobicity if mainly hydrophobic binding is involved, and the solubilization capacity of the micelles should increase with its size. The fact that higher generation dendrimers are able to solubilize a larger number of guest molecules was clearly demonstrated: ${ }^{73}$ The amount of pyrene solubilized in the core of dendrimers derived from 4,4-bis(4'-hydroxyphenyl)pentanol was found to increase with the generation number of the dendrimers to reach $2.85 \mathrm{Py} /$ micelle for the G3 molecules. A similar observation was reported earlier by Pistolis et al. for PAMAM dendrimers: ${ }^{74}$ The amounts of pyrene solubilized in $10^{-2} \mathrm{M}$ aqueous solutions of the dendrimers varied with the generation number from $G 0$ (MW $=516$ with 2 cavities, $1.1 \times 10^{-}$ ${ }^{6} \mathrm{M}$ Py solubilized) to $G 1\left(\mathrm{MW}=1429\right.$ with 6 cavities, $2.64 \times 10^{-6} \mathrm{M}$ Py), and $G 2(\mathrm{MW}=$ 3250 with 14 cavities, $7.55 \times 10^{-6} \mathrm{M}$ Py). Thus the amount solubilized increases linearly with both the molecular weight and the number of cavities as the dendrimer grows in size.

The size of dendritic micelles not only affects the solubilization capacity for guest molecules, but also their release rate to the surroundings. Ooya et al. ${ }^{88}$ investigated the influence of the generation number of polyglycerol dendrimers on the solubilization and 
release of the drug paclitaxel. The solubility of paclitaxel in $G 3, G 4$, and $G 5$ micelles at a concentration of $10 \% \mathrm{w} / \mathrm{w}$ was respectively $270-, 370$ - and 430 -fold higher than in water $(0.3 \mu \mathrm{g} / \mathrm{mL})$. While the time required for complete release of the drug was independent of the generation number, the initial rate of release was strongly dependent on dendrimer generation, being highest for generation $G 5$ and lowest for $G 3$ molecules. This difference was attributed to the solubilization power of the dendrimer micelles, the $G 5$ micelles with the highest solubilization power having also the highest release rate.

The influence of both solute and micelle structure on the solubilization process was demonstrated by Santo and Fox ${ }^{81}$ in their study of hydrogen bonding interactions between PAMAM dendrimers and various biological molecules using four different dendrimers 1-4 (Figure 2.14). Dendrimers 1-4 all contained internal amido groups, but only $\mathbf{1}$ and $\mathbf{2}$ had free peripheral amino groups. The biological guest molecules $\mathbf{5}$ investigated included pyridine, quinoline, quinazoline, and trimethadione. ${ }^{1} \mathrm{H}$ NMR chemical shifts were used to determine the location and strength of hydrogen bonding sites between the host and guest molecules. Because of its small size, pyridine was able to form H-bonds with both internal amido groups and external amino groups of dendrimer $\mathbf{1}$. Both the amine and amide groups of dendrimer 2 displayed shifts in proton resonances, however no interactions were observed for internal amide protons. The migration of pyridine molecules to the internal amide groups was apparently more difficult due to steric limitations. Dendrimer 3, on the other hand, interacted strongly with pyridine only through the amide protons. 

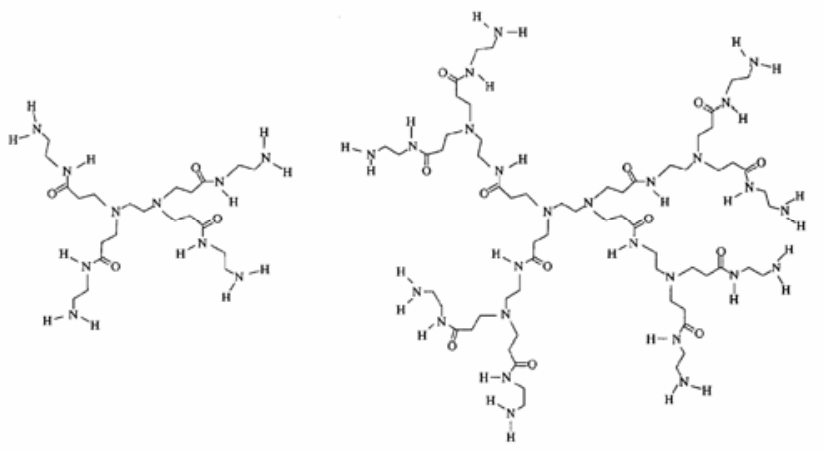

(1)

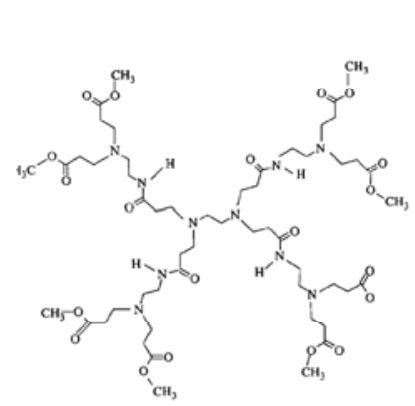

(3)

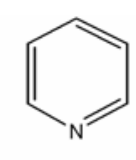

Pyridine<smiles>c1ccc2ncccc2c1</smiles>

Quinoline
(2)

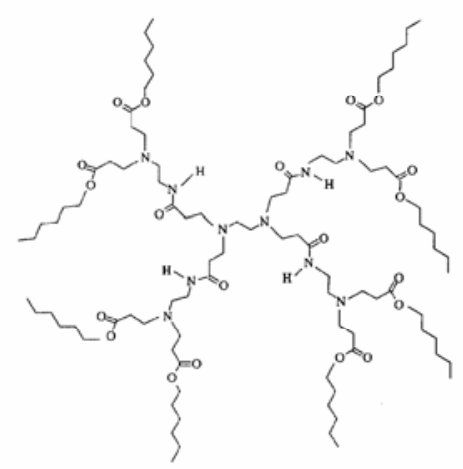

(4)<smiles>c1ccc2ncncc2c1</smiles>

Quinazoline<smiles>CN1CCCC1c1cccnc1</smiles>

Trimethadione

(5)

Figure 2.14. Structure of modified PAMAM dendrimers (1-4) used as hosts, (5) biological guest molecules. ${ }^{81}$ 
The association constants $K_{\mathrm{a}}\left(1.31 \mathrm{M}^{-1}\right)$ for pyridine and $\mathbf{1}$ were greater than for compounds 3 and $4\left(1.11 \mathrm{M}^{-1}\right.$ and $\left.1.06 \mathrm{M}^{-1}\right)$, presumably because migration of the pyridine molecules to the internal amide groups was hindered by the bulky peripheral ester groups of these micelles. Quinazoline and quinoline were able to form H-bonds with 3, however these interactions were very weak as indicated by their respective $K_{\mathrm{a}}$ values of $0.83 \mathrm{~L} / \mathrm{mol}$ and 0.69 L/mol. No interactions between these guest molecules and $\mathbf{4}$ were detected, again presumably because of the long outer alkyl chains hindering the percolation of these large molecules to the interior. With trimethadione, no significant hydrogen bonding was observed for any of the dendrimers.

2.5.3 Release of Guest Molecules. Unimolecular micelles have been used as devices for the release of hydrophobic compounds. ${ }^{73,89}$ Studies have shown that these highly branched structures are able to release hydrophobic drugs in a sustained manner, as release has been found at times to continue for several hours. ${ }^{73,89}$ In the previous section it was mentioned that the release of hydrophobic molecules from dendritic micelles is dependent on both the architecture of the host and the size and shape of the guest molecules. The release of hydrophobic compounds from amphiphilic dendritic polymers can also be influenced by the type of host-guest interactions involved. While weaker host-guest interactions like hydrophobic attractions allow the almost complete release of the encapsulated molecules from the micelle, partial release of the compounds has been found to occur when stronger interactions (hydrogen and ionic bonding) are involved. Release studies of lidocaine from a dendritic micelle with an interior core consisting of lauroyl ester of mucic acid and an exterior shell of methoxy-terminated poly(ethylene glycol) chains have been performed in an aqueous medium..$^{90}$ At $\mathrm{pH} 7$ lidocaine is hydrophobic and the interactions between the core 
of the micelle and the drug are hydrophobic. The release profile shows sustained character and it is observed that almost all the drug molecules encapsulated are released after about 30 hours (Figure 2.15).

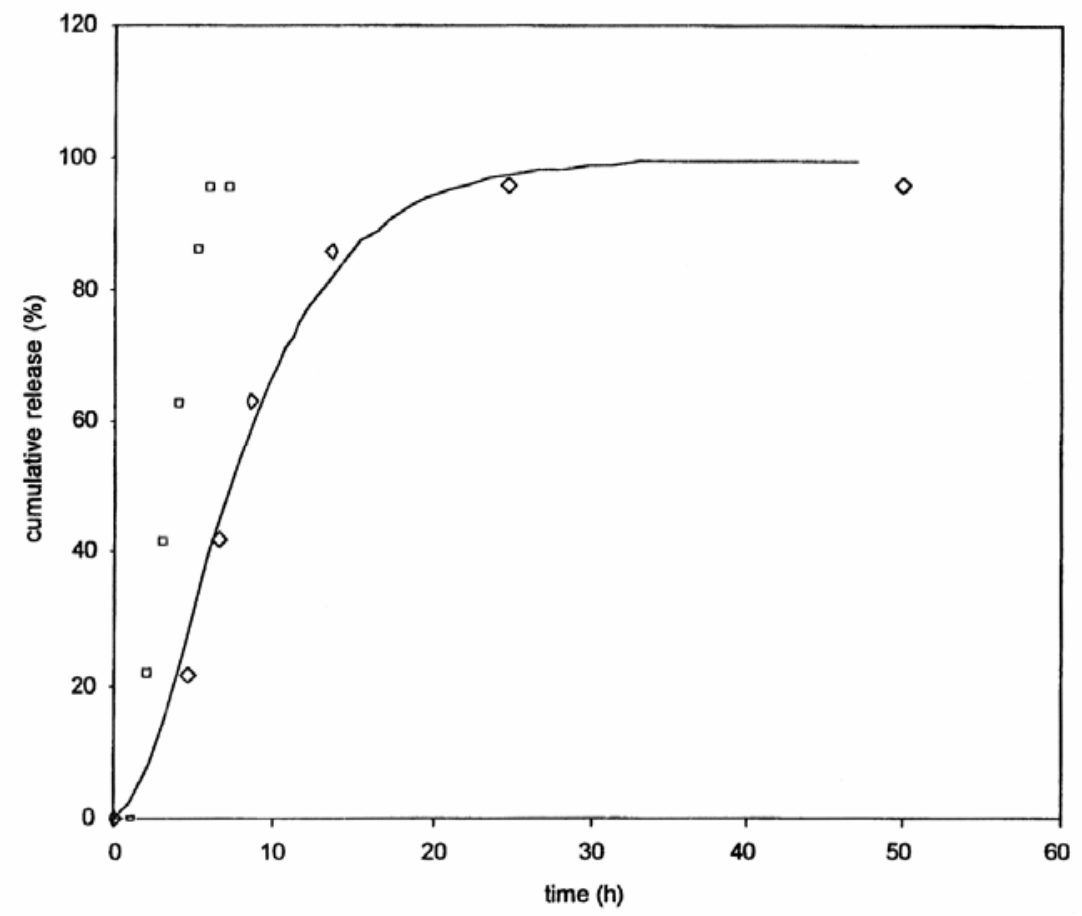

Figure 2.15. Cumulative release of free lidocaine $(\square)$ and polymer-encapsulated lidocaine $(\diamond)$ showing complete release of encapsulated drug. ${ }^{90}$

Encapsulation studies of ibuprofen in PAMAM dendrimers end-functionalized with $-\mathrm{NH}_{2}$ groups displayed two types of host-guest interactions: hydrophobic interactions between the drug molecules and the hydrophobic core, and ionic interactions between the carboxylic group of the drug and the amino end groups of the PAMAM dendrimer. ${ }^{91}$ It was observed in the release studies that $100 \%$ of the drug was released within 6 hours when in 
vitro release studies were performed with pure ibuprofen in an RPMI 1640 culture medium (a bicarbonate buffering system containing amino acids and vitamins) (Figure 2.16). The release of the drug from the dendrimer was appreciably slower, with $70 \%$ and $80 \%$ of the drug being released from $G 3$ and $G 4$ dendrimers, respectively, after 8-9 hours. It was demonstrated that due to the small size of the $G 3$ dendrimer, ibuprofen molecules were not solubilized in the hydrophobic interior of the micelle but rather formed complexes via ionic interactions with the peripheral amino groups of the dendrimer. In the $G 4$ micelle, however, a small fraction of ibuprofen $(\sim 18 \%)$ was also encapsulated within the hydrophobic voids of the dendritic core, leading to the higher release of the drug from the $G 4$ dendritic micelles.

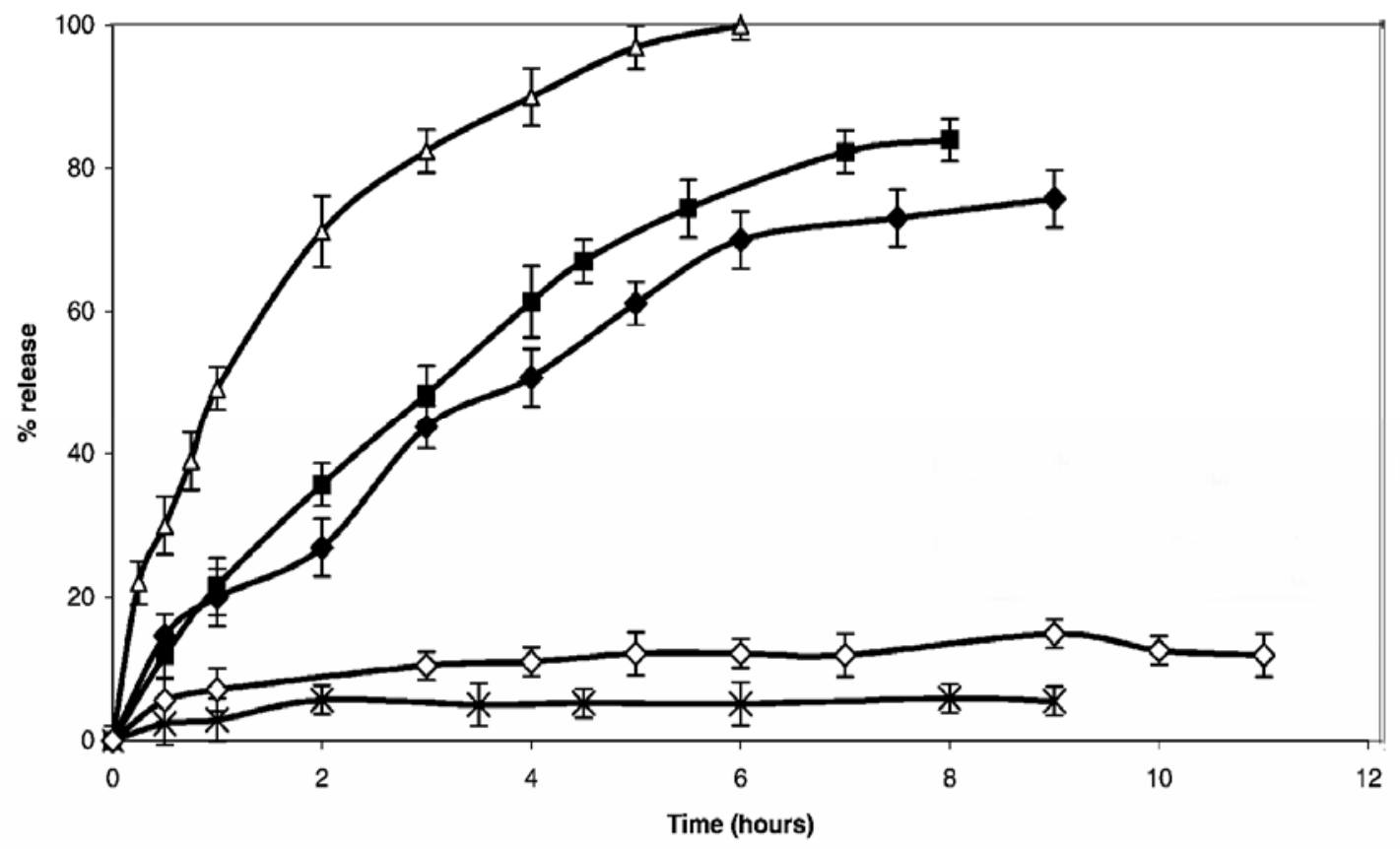

Figure 2.16. Cumulative profiles for the release of ibuprofen. Release of pure drug $(\Delta)$, drug in $G 4$ dendrimer ( $\bullet$ ), drug in $G 3$ dendrimer ( $\bullet$ ) all in culture medium; drug in $G 4$ dendrimer in deionized water $(\diamond)$; drug in $G 4$ dendrimer in methanol $(\times) .{ }^{91}$ 
In methanol and deionized water, less than $15 \%$ of the drug was released from the $G 4$ dendritic micelle even after 9 hours. This suggests that the stronger the drug-dendrimer complex, the lower the fraction of drug released.

\subsection{Conclusions}

Amphiphilic dendritic polymers possess significant advantages over small molecule surfactant and block copolymer micelles. Dendritic micelles can remain non-associated (unimolecular) under most solvency conditions and do not display a CMC, as their structure is stabilized by covalent bonds. Extensive control is achieved over the solubilization and selfassembly behaviors of dendritic amphiphiles by varying structural parameters such as the size (generation number), internal and superficial chemical functionality, and architecture of the molecules. In this chapter, we attempted to give a concise account on the interfacial properties of these macromolecules, with emphasis on solubilization, release and selfassembly at the air-water interface. To provide a better understanding, an overview of the synthesis, internal structure, and interfacial properties of amphiphilic dendritic polymers was also presented. Each dendritic polymer family has distinct properties, dendrimers and dendrigraft polymers displaying the most similarities, albeit on a very different size scale. Considering their unique features, it is not surprising to see the large number of applications emerging for amphiphilic dendritic polymers in different areas.

\subsection{References}

1. Dykes, G. M. J. Chem. Technol. Biotechnol. 2001, 76, 903. 
2. Ghosh, S. K.; Kawaguchi, S.; Jinbo, Y.; Izumi, Y.; Yamaguchi, K.; Taniguchi, T.; Nagai, K.; Koyama, K. Macromolecules 2003, 36, 9162.

3. Moorefield, C. N; Newkome, G. R. C. R. Chimie 2003, 6, 715.

4. Teerststra, S. J.; Gauthier, M. Prog. Polym. Sci. 2004, 29, 277.

5. Tomalia, D. A.; Fréchet, J. M. J. In Dendrimers and other Dendritic Polymers; Fréchet, J. M. J.; Tomalia, D. A. Eds; Wiley: West Sussex, 2001, Chapter 1.

6. de Gennes, P. G.; Hervet, H. J. Physique-Lett. 1983, 44, 351.

7. Tomalia, D. A.; Durst, H. D. Top. Curr. Chem. 1993, 165, 193.

8. Hawker, C. J.; Wooley, K. L.; Fréchet, J. M. J. J Chem. Soc. Perkin. Trans. Part 1, 1993, 1287.

9. Lescanec, R. L.; Muthukumar, M. Macromolecules 1990, 23, 2280.

10. Mansfield, M. L.; Klushin, L. I. Macromolecules 1993, 26, 4262.

11. Murat, M.; Grest, G. S. Macromolecules 1996, 29, 1278.

12. Pötschke, D.; Ballauff, M.; Lindner, P.; Fischer, M.; Vögtle, F. Macromolecules 1999, 32,4079 .

13. Giupponi, G.; Buzza, D. M. A. Macromolecules 2002, 35, 9799.

14. Tomalia, D. A.; Hall, M.; Hedstrand, D. M. J. Am. Chem. Soc. 1987, 109, 1601.

15. Tomalia, D. A.; Naylor, A. M.; Goddard III, W. A. Angew. Chem. Int. Ed. Engl. 1990, $29,138$.

16. Fréchet, J. M. J.; Hawker, C. J.; Gitsov, I.; Leon, J. W. J. M. S. - Pure Appl. Chem. 1996, A33, 1399.

17. Mourey, T. H.; Turner, S. R.; Rubinstein, M.; Fréchet, J. M. J.; Hawker, C. J.; Wooley, K. L. Macromolecules 1992, 25, 2401. 
18. Naylor, A. M.; Goddard III, W. A.; Kiefer, G. E.; Tomalia, D. A. J. Am. Chem. Soc. 1989, 111, 2339.

19. (a) Malenfant, P. R. L.; Fréchet, J. M. J. In Dendrimers and other Dendritic Polymers; Fréchet, J. M. J.; Tomalia, D. A. Eds; Wiley: West Sussex, 2001, Chapter 7 (b) Gitsov, I. In Advances in Dendritic Molecules, Newkome, G. R. Ed; Elsevier, Amsterdam, 2002, vol. 5, p. 45.

20. Gauthier, M.; Möller, M.; Burchard, W. Macromol. Symp. 1994, 77, 43.

21. Frank, R. S.; Merkle, G.; Gauthier, M. Macromolecules 1997, 30, 5397.

22. Choi, S.; Briber, R. M.; Bauer, B. J.; Topp, A.; Gauthier, M.; Tichagwa. L. Macromolecules 1999, 32, 7879.

23. Gauthier, M.; Li, W.; Tichagwa, L. Polymer 1997, 38, 6363.

24. Flory, P. J. J. Am. Chem. Soc. 1952, 74, 2718.

25. Thompson, D. S.; Markoski, L. J.; Moore, J. S.; Sendijarevic, I.; Lee, A.; McHugh, A. J. Macromolecules 2000, 33, 6412.

26. Parker, D.; Feast, W. J. Macromolecules 2001, 34, 2048.

27. Mori, H.; Müller, A. H. E. Top. Curr. Chem. 2003, 228, 1.

28. Voit, B. I. C. R. Chimie 2003, 6, 821.

29. Wooley, K. L.; Fréchet, J. M. J.; Hawker, C. J. Polymer 1994, 35, 4489.

30. Xu, K.; Peng, H.; Sun, Q.; Dong, Y.; Salhi, F.; Luo, J.; Chen, J.; Huang, Y.; Zhang, D.; Xu, Z.; Tang, B. Z. Macromolecules 2002, 35, 5821.

31. Ishizu, K.; Mori, A. Macromol. Rapid Commun. 2000, 21, 665.

32. Voit, B. J. Polym. Sci. Part A: Polym. Chem. 2000, 38, 2505.

33. Jikei, M.; Kakimoto, M. Prog. Polym. Sci. 2001, 26, 1233. 
34. Prosa, T. J.; Bauer, B. J.; Amis, E. J.; Tomalia, D. A.; Scherrenberg, R. J. Polym. Sci. Part B: Polym. Phys. 1997, 35, 2913.

35. Ishizu, K.; Mori, A. Polym. Int. 2001, 51, 50.

36. Antonietti, M.; Bremser, W.; Schmidt, M. Macromolecules 1990, 23, 3796.

37. Bantle, S.; Schmidt, M.; Burchard, W. Macromolecules 1982, 15, 1604.

38. Mössmer, S.; Spatz, J. P.; Möller, M.; Aberle, T.; Schmidt, J.; Burchard, W. Macromolecules 2000, 33, 4791.

39. Newkome, G. R.; Moorefield, C. N.; Baker, G. R.; Johnson, A. L.; Behera, R. K.; Angew. Chem. Int. Ed. Engl. 1991, 30, 1176.

40. Twyman, L. J.; Beezer, A. E.; Esfand, R.; Hardy, M. J.; Mitchell, J. C. Tetrahedron Lett. 1999, 40, 1743.

41. Ihre, H.; Hult, A.; Fréchet, J. M. J.; Gitsov, I. Macromolecules 1998, 31, 4061.

42. Kim, C.; Son, S.; Kim, B. J. Organomet. Chem. 1999, 588, 1.

43. Zhang, X.; Haxton, K. J.; Ropartz, L.; Cole-Hamilton, D. J.; Morris, R. E. J. Chem. Soc. Dalton Trans. 2001, 3261.

44. Haag, R.; Stumbé, J.-F.; Sunder, A.; Frey, H.; Hebel, A. Macromolecules 2000, 33, 8158.

45. Liu, H.; Jiang, A.; Guo, J.; Uhrich, K. E. J. Polym. Sci. Part A: Polym. Chem. 1999, 37, 703.

46. Hedrick, J. L.; Trollsås, M.; Hawker, C. J.; Atthoff, B.; Claesson, H.; Heise, A.; Miller, R. D. Macromolecules 1998, 31, 8691.

47. Zhao, Y.-L.; Cai, Q.; Jiang, J.; Shuai, X.-T.; Bei, J.-Z.; Chen, C.-F.; Xi, F. Polymer 2002, 43, 5819 . 
48. Kee, R. A.; Gauthier, M. Macromolecules 2002, 35, 6526.

49. Gauthier, M.; Li, J.; Dockendorff, J. Macromolecules 2003, 36, 2642.

50. Gauthier, M.; Tichagwa, L.; Downey, J. S.; Gao, S. Macromolecules 1996, 29, 519.

51. Taton, D.; Cloutet, E.; Gnanou, Y. Macromol. Chem. Phys. 1998, 199, 2501.

52. Angot, S.; Taton, D.; Gnanou, Y. Macromolecules 2000, 33, 5418.

53. Gong, C.; Fréchet, J. M. J. J. Polym. Sci. Part A: Polym. Chem. 2000, 38, 2970.

54. Weberskirch, R.; Hettich, R.; Nuyken, O.; Schmaljohann, D.; Voit, B. Macromol. Chem. Phys. 1999, 200, 863.

55. (a) Gitsov, I.; Wooley, K. L.; Fréchet, J. M. J. Angew. Chem. Int. Ed. Engl. 1992, 31, 1200. (b) Gitsov, I.; Wooley, K. L.; Hawker, C. J.; Ivanova, P. T.; Fréchet, J. M. J. Macromolecules 1993, 26, 5621.

56. Gitsov, I.; Fréchet, J. M. J. Macromolecules 1993, 26, 6536.

57. van Hest, J. C. M.; Delnoye, D. A. P.; Baars, M. W. P. L.; Elissen-Román, C.; van Genderen, M. H. P.; Meijer, E. W. Chem. Eur. J. 1996, 2, 1616.

58. Chang, Y.; Kwon, Y. C.; Lee, S. C.; Kim, C. Macromolecules 2000, 33, 4496.

59. Chang, Y.; Kim, C. J. Polym. Sci. Part A: Polym. Chem. 2001, 39, 918.

60. Hann, R. A. In Langmuir-Blodgett Films; Roberts, G. Ed; Plenum: New York, 1990, Chapter 2.

61. Petty, M. C.; Barlow, W. A. In Langmuir-Blodgett Films; Roberts; G. Ed; Plenum: New York, 1990, Chapter 3.

62. Schenning, A. P. H. J.; Elissen-Román, C.; Weener, J.-W.; Baars, M. W. P. L.; van der Gaast, S. J.; Meijer, E. W. J. Am. Chem. Soc. 1998, $120,8199$. 
63. Cardullo, F.; Diederich, F.; Echegoyen, L.; Habicher, T.; Jayaraman, N.; Leblanc, R. M.; Stoddart, J. F.; Wang, S. Langmuir 1998, 14, 1955.

64. Felder, D.; Gallani, J.-L.; Guillon, D.; Heinrich, B.; Nicoud, J.-F.; Nierengarten, J.-F. Angew. Chem. Int. Ed. 2000, 39, 201.

65. Nierengarten, J.-F.; Eckert, J.-F.; Rio, Y.; del Pilar Carreon, M.; Gallani, J.-L.; Guillon, D. J. Am. Chem. Soc. 2001, 123, 9743.

66. Sui, G.; Mabrouki, M.; Ma, Y.; Micic, M.; Leblanc, R. M. J. Colloid Interface Sci. 2002, 250, 364 .

67. Sui, G.; Micic, M.; Huo, Q.; Leblanc, R. M. Langmuir 2000, 16, 7847.

68. Sui, G.; Micic, M.; Huo, Q.; Leblanc, R. M. Colloids Surfaces A: Physicochem. Eng. Aspects 2000, 171, 185.

69. Tanaka, K.; Dai, S.; Kajiyama, T.; Aoi, K.; Okada, M. Langmuir 2003, 19, 1196.

70. Zhai, X.; Peleshanko, S.; Klimenko, N. S.; Genson, K. L.; Vaknin, D.; Vortman, M. Y.; Shevchenko, V. V.; Tsukruk, V. V. Macromolecules 2003, 36, 3101.

71. Ponomarenko, S. A.; Boiko, N. I.; Shibaev, V. P.; Magonov, S. N. Langmuir 2000 16, 5487.

72. Newkome, G. R.; Moorefield, C. N.; Baker, G. R.; Saunders, M. J.; Grossman, S. H. Angew. Chem. Int. Ed. Engl. 1991, 30, 1178.

73. Liu, M.; Kono, K.; Fréchet, J. M. J. J. Controlled Release 2000, 65, 121.

74. Pistolis, G.; Malliaris, A.; Paleos, C. M.; Tsiourvas, D. Langmuir 1997, 13, 5870.

75. Gitsov, I.; Lambrych, K. R.; Remnant, V. A.; Pracitto, R. J. Polym. Sci. Part A: Polym. Chem. 2000, 38, 2711. 
76. Jansen, J. F. G. A.; de Brabander-van den Berg, E. M. M.; Meijer, E. W. Science 1994, $266,1226$.

77. Jansen, J. F. G. A.; Meijer, E. W.; de Brabander-van den Berg, E. M. M. J. Am. Chem. Soc. 1995, 117, 4417.

78. Jansen, J. F. G. A.; Meijer, E. W.; de Brabander-van den Berg, E. M. M. Macromol. Symp. 1996, 102, 27.

79. Newkome, G. R.; Woosley, B. D.; He, E.; Moorefield, C. N.; Güther, R.; Baker, G. R.; Escamilla, G. H.; Merrill, J.; Luftmann, H. Chem. Commun. 1996, 2737.

80. Zimmerman, S. C.; Wang, Y.; Bharathi, P.; Moore, J. S. J. Am. Chem. Soc. 1998, 120, 2172.

81. Santo, M.; Fox, M. A. J. Phys. Org. Chem. 1999, 12, 293.

82. Cooke, G.; Sindelar, V.; Rotello, V. M. Chem. Commun. 2003, 752.

83. Krämer, M.; Stumbé, J. -F.; Türk, H.; Krause, S.; Komp, A.; Delineau, L.; Prokhorova, S.; Kautz, H.; Haag, R. Angew. Chem. Int. Ed. 2002, 41, 4252.

84. Baars, M. W. P. L.; Kleppinger, R.; Koch, M. H. J.; Yeu, S.-L.; Meijer, E. W. Angew. Chem. Int. Ed. 2000, 39, 1285.

85. Beezer, A. E.; King, A. S. H.; Martin, I. K.; Mitchel, J. C.; Twyman, L. J.; Wain, C. F. Tetrahedron 2003, 59, 3873.

86. Baars, M. W. P. L.; Froehling, P. E.; Meijer, E. W. Chem. Commun. 19971959.

87. Schenning, A. P. H. J.; Peeters, E.; Meijer, E. W. J. Am. Chem. Soc. 2000, 122, 4489.

88. Ooya, T.; Lee, J.; Park, K. J. Controlled Release 2003, 93, 121.

89. Jones, M.-C.; Ranger, M; Leroux, J.-C. Bioconjugate Chem. 2003, 14, 774.

90. Liu, H.; Farrell, S.; Uhrich, K. J. Controlled Release 2000, 68, 167. 
91. Kolhe, P.; Misra, E.; Kannan, R. M.; Kannan, S.; Lieh-Lai, M. Int. J. Pharm. 2003, $259,143$. 


\section{Chapter 3}

Self-assembly of Arborescent Polystyrene-graft-

Poly(ethylene oxide) Copolymers at the Air-water

Interface 


\subsection{Abstract}

Atomic force microscopy (AFM) imaging was used to investigate the influence of molecular structure and composition on the formation of Langmuir monolayers by arborescent polystyrene-graft-poly(ethylene oxide) (PS-g-PEO) copolymers at the air-water interface. These amphiphilic dendritic micelles are characterized by a highly branched hydrophobic PS core surrounded by a thin shell of flexible PEO chains. Even without compression, these molecules associated spontaneously at the air-water interface and formed stable monolayers which were easily transferred onto solid substrates as Langmuir-Blodgett films. Copolymers incorporating long PS segments in the core (side chain $M_{\mathrm{w}} \approx 30000$ ), with a low core branching functionality $\left(f_{\mathrm{w}}=62\right)$ and a PEO content $\geq 22 \%$ by weight spontaneously formed ribbon-like superstructures, while more rigid molecules with a high core branching functionality $\left(f_{\mathrm{w}}=270\right)$ only displayed a low level of association. Copolymers based upon either low or high branching functionality cores with a PEO content $\leq 15 \%$ by weight formed island-like clusters with a broad size distribution due to coalescence and dewetting. For copolymers incorporating stiffer cores with very short PS chains $\left(M_{\mathrm{w}} \approx 5000\right)$, no association was observed for a branching functionality $f_{\mathrm{w}}=170$ while molecules with a much higher branching functionality $\left(f_{\mathrm{w}}=3400\right)$ formed island-like clusters on the water surface. The different behaviors of the copolymers were rationalized in terms of the influence of composition and of the structure of the arborescent PS cores on the assembly process. 


\subsection{Introduction}

Much attention has been devoted to the self-assembly of dendritic molecules to superstructures of controllable topologies. ${ }^{1}$ These supramolecular assemblies can be formed via covalent bonding between a large dendrimer core and smaller dendrimers forming a corona as in the case of tecto(dendrimers), ${ }^{2}$ but more commonly through non-covalent interactions such as hydrogen bonding, ${ }^{3}$ by coordination to a central metal cation, ${ }^{4}$ or under the influence of van der Waals forces. ${ }^{5}$

Amphiphilic dendritic systems such as surface-modified dendrimers with a hydrophobic core of large dimensions as compared to the hydrophilic corona have the ability to form supramolecular assemblies at interfacial boundaries. The incorporation of such assemblies into ordered ultrathin films is becoming an important issue, as dendritic thin films offer interesting application possibilities. ${ }^{6}$ Different methods have been used to produce thin films, ${ }^{7}$ but the Langmuir balance technique is still widely applied because it also enables the study of orientation and conformational changes of the amphiphiles at the air-water interface. Furthermore, this technique often provides control over orientation on the molecular scale, and over the topology of the assemblies.

The behavior of linear PS- $b$-PEO copolymer systems at the air-water interface has been studied extensively by the Langmuir balance technique. ${ }^{8}$ The same method has also been used to study the conformation of PS-PEO star-block and dendrimer-like copolymers of low branching functionalities $\left(f_{\mathrm{w}}=3-6\right)$ at the air-water interface, ${ }^{9,10}$ but not for branched amphiphilic copolymers with very high branching functionalities such as the arborescent PS$g$-PEO molecules. The present report describes the self-assembly behavior of PS-g-PEO arborescent copolymers at the air-water interface using the Langmuir balance technique 
without compression, by relating the topology of the Langmuir-Blodgett films observed by AFM imaging to copolymer structure. The influence of branching functionality as well as the core and corona chain lengths on the association of these dendritic micelles is discussed.

\subsection{Experimental Section}

3.3.1 Materials. Detailed procedures for the synthesis and the characterization of the core-shell arborescent copolymers used in this study have been described previously. ${ }^{11}$ The materials were characterized in terms of side chain molecular weight for the PS cores using size exclusion chromatography, while the absolute weight-average molecular weight $\left(M_{\mathrm{w}}\right)$ of the cores was determined from static light scattering measurements. The composition of the

copolymers was determined by FTIR and ${ }^{1} \mathrm{H}$ NMR spectroscopy analysis,${ }^{11 \mathrm{~b}}$ and the average molecular weight of the individual PEO chains $M_{\mathrm{w}(\mathrm{PEO})}$ was calculated by dividing the increase in molecular weight upon adding the shell by the branching functionality $\left(f_{\mathrm{w}}\right)$ of the core.

Dynamic light scattering measurements were used to estimate the hydrodynamic diameter of the arborescent polystyrene cores and the core-shell copolymers in $\mathrm{CHCl}_{3}$. The samples were dissolved at a concentration of $0.5 \mathrm{mg} / \mathrm{mL}$, sonicated for $30 \mathrm{~min}$ in a water bath, and filtered through $0.45 \mu \mathrm{m}$ pore size poly(tetrafluoroethylene) (PTFE) membrane filters. The measurements were done at $25{ }^{\circ} \mathrm{C}$ and at a scattering angle of $90^{\circ}$ on a Brookhaven BI-200 SM light scattering goniometer equipped with a BI-2030AT 201-channel correlator and a Lexel 2-W argon ion laser $(514.5 \mathrm{~nm})$. The hydrodynamic diameter was calculated from the z-average diffusion coefficient $\left(D_{z}\right)$ derived from second-order analysis of the normalized dynamic light scattering correlation function $\mathrm{g}(\tau)$. 
3.3.2 Langmuir Trough Experiments. Langmuir monolayers were obtained with a custom-built Langmuir balance consisting of a shallow rectangular PTFE trough $(43 \times 15$ $\mathrm{cm}^{2}$ ). The trough was enclosed in a glass case with a removable lid, to prevent airborne contamination, and supported on a Micro-g air table to reduce vibrations. After sonication for $30 \mathrm{~min}, 100 \mu \mathrm{L}$ of copolymer solution in chloroform (Merck, HPLC grade) at a concentration of $0.5 \mathrm{mg} / \mathrm{mL}$ were spread with a microsyringe on the surface of the Milli-Q water $(18 \mathrm{M} \Omega . \mathrm{cm})$ subphase in the trough. The temperature of the subphase was maintained at $22.0 \pm 0.5^{\circ} \mathrm{C}$. After the transfer of each copolymer film, the subphase was removed and the trough was cleaned with a NOCHROMIX ${ }^{\circledR}$ solution in concentrated sulfuric acid.

3.3.3 Atomic Force Microscopy (AFM). Mica plates and silicon wafers were used as transfer substrates for the Langmuir-Blodgett films. The mica plates were dipped in chloroform for $15 \mathrm{~min}$ and dried before cleavage. The silicon wafers were cleaned with NOCHROMIX $^{\circledR}$ in concentrated sulfuric acid, and rinsed with Milli-Q water before use. A Lauda FL-1 Filmlift was used to immerse the substrate into the subphase before spreading the polymer solution. After 15 min (to allow for solvent evaporation), the substrate was pulled through the interface at a rate of $3 \mathrm{~mm} / \mathrm{min}$ to transfer the monolayer onto the surface. The Langmuir-Blodgett films were dried at room temperature for $2-3$ days and characterized with a Nanoscope III Scanning Probe Microscope (Digital Instrument Inc., Santa Barbara, CA), operated in the tapping mode with $125 \mu \mathrm{m}$ length NanoProbe ${ }^{\mathrm{TM}}$-TESP silicon cantilever tips having a resonant frequency between $260-410 \mathrm{kHz}$. 


\subsection{Results and Discussion}

Research on Langmuir monolayers of dendritic amphiphiles has focused mainly on compounds in the dendrimer family, because of their globular and well-defined architecture. Since the transfer of monolayers obtained from these compounds onto solid substrates can be a challenge due to their low level of surface binding and lack of entanglements, LangmuirBlodgett films have been obtained mainly from dendrimers modified on their surface with either long alkyl or polymer chains. ${ }^{5,12,13}$ For example, amphiphiles based on polypropylenimine dendrimers of different generations modified on their surface with hydrophobic chains formed stable Langmuir monolayers at the air-water interface. ${ }^{5}$ These films could be transferred to form Langmuir-Blodgett films on hydrophilic glass substrates, but the transfer efficiency of the monolayers was low. In another study, the transfer onto silicon wafers of monolayers prepared from generation three (G3) polyamidoamine (PAMAM) hemispheric dendrons modified on their surface with dodecyl chains was investigated. ${ }^{14}$ Hydrophilically modified silicon wafers remained mostly bare, as confirmed by AFM measurements, presumably due to slippage of the PAMAM molecules from the substrate during lifting. This problem was avoided by using hydrophobically modified silicon surfaces and by employing a horizontal lifting method, whereby the substrate was just brought in contact with the amphiphilic molecules at the surface. It seems that the dodecyl chains not only increased the hydrophobic/hydrophilic balance but also the degree of adhesion by augmenting the number of surface-binding groups.

Amphiphilic arborescent copolymers are dendritic molecules synthesized from polymeric building blocks according to a generation-based scheme that relies on cycles of substrate functionalization and grafting. ${ }^{15}$ Arborescent PS- $g$-PEO copolymers are an example 
of such dendritic amphiphiles synthesized from successive anionic grafting reactions. ${ }^{11}$ These micelles incorporate a large core surrounded by a thin corona layer, and are therefore expected to display self-assembly. It will be shown that the flexible polymeric chains in the corona of these copolymers provide sufficient entanglements and binding interactions for adhesion onto solid substrates. For dendritic micelles derived from hyperbranched and dendrimer cores, interaction control can be achieved by varying the generation number of the core, the length of the corona chains, and the degree of substitution of the chain ends. The interactions of arborescent amphiphiles can be modified likewise through branching functionality (generation) and composition variations, but also by adjusting the length of the chains in the core, to control the structural rigidity of the molecules.

3.4.1 Arborescent PS-g-PEO Copolymers. Most of the amphiphilic core-shell arborescent copolymers used in this study were synthesized from twice-grafted (G1) polystyrene cores (Scheme 3.1). Comb-branched or G0 PS homopolymers were first synthesized by grafting PS side chains with a weight-average molecular weight $\left(M_{\mathrm{w}}\right)$ of either 5000 or 30000 onto chloromethylated linear PS chains $\left(M_{\mathrm{w}} \approx 5000\right) .{ }^{1 \mathrm{~b}}$ The $G 1$ cores were synthesized by chloromethylation and grafting of the $G 0$ substrates with PS side chains having a molecular weight similar to the $G 0$ substrates $\left(M_{\mathrm{w}} \approx 5000\right.$ or 30000$)$ and carrying a terminal acetal functionality. Sample G1-14PS-LB-19 was obtained by grafting acetalterminated PS side chains with $M_{\mathrm{w}} \approx 14000$ onto the $G 0$ substrate with $M_{\mathrm{w}} \approx 5000$ side chains. Sample G3-5PS-HB-43 was derived from a $G 3$ core obtained from four grafting reactions with $M_{\mathrm{w}} \approx 5000$ side chains. The polystyrene cores are broadly defined as having either a low branching functionality (LB, $f_{\mathrm{w}}=44$ or 62$)$ or a high branching functionality 

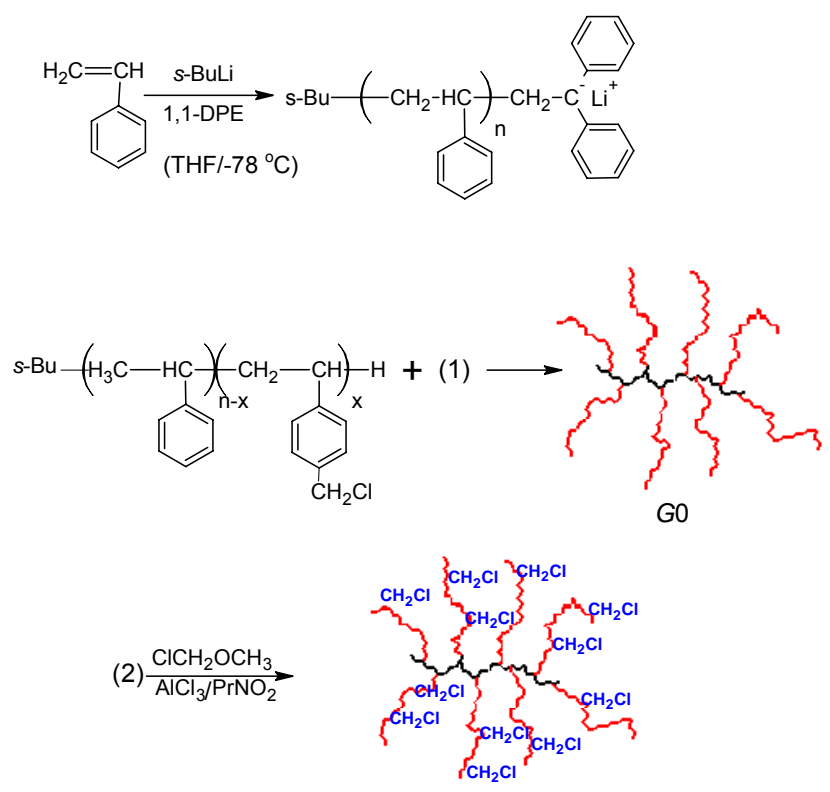

(3)
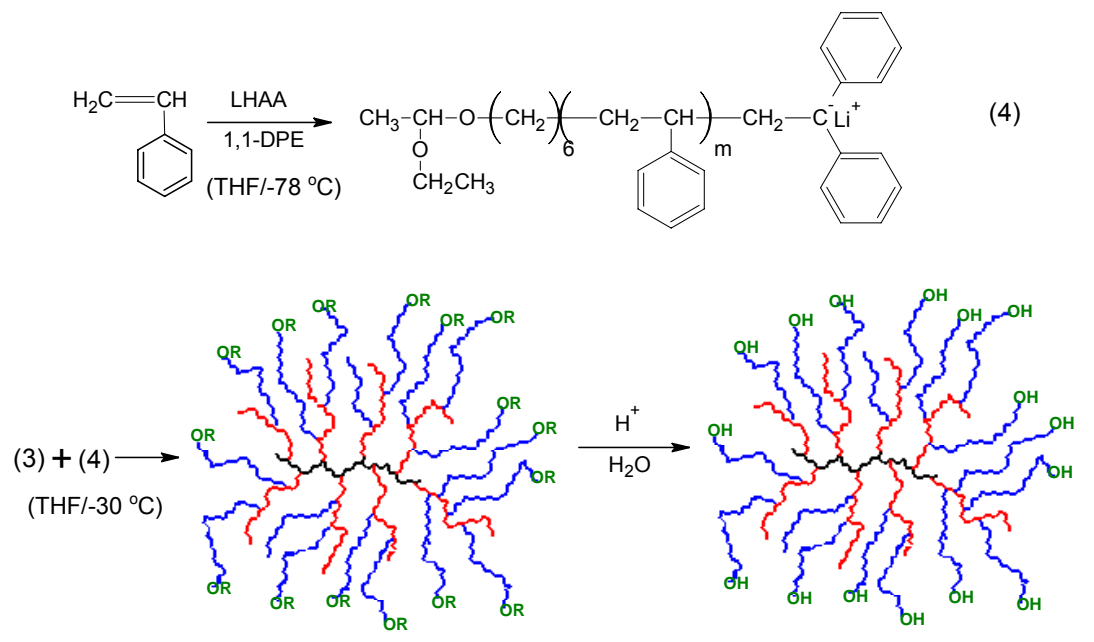

G1

(5)

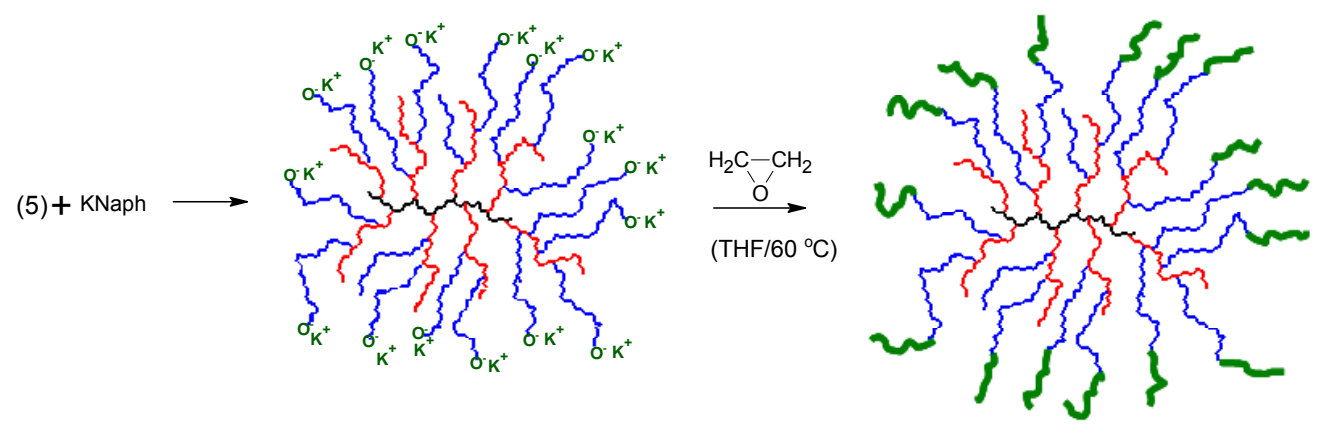

Scheme 3.1. Reaction scheme for the synthesis of arborescent PS- $g$-PEO copolymers. 
(HB, $f_{\mathrm{w}}=170,270$, or 3400 ). The branching functionality is defined as the number of side chains added in the last grafting cycle and is calculated from the equation

$$
f_{w}=\frac{M_{w}(G)-M_{w}(G-1)}{M_{w}^{b r}}
$$

where $M_{w}(G)$ and $M_{w}(G-1)$ are the absolute weight-average molecular weights for polymers of generation $G$ and of the previous generation, respectively, and $M_{w}^{b r}$ is the molecular weight of the acetal-terminated side chains grafted in the last step of the core synthesis. The high branching functionality (HB) molecules should be more rigid and less deformable than the molecules with a low branching (LB) functionality.

The PEO copolymers were obtained by acid-catalyzed hydrolysis of the terminal acetal functionalities, titration with a base (potassium naphthalide), and addition of ethylene oxide to the polyfunctional anionic initiator thus obtained (Scheme 3.1). ${ }^{11}$ A $100 \%$ activation and initiation efficiency was assumed. Before adding the base, a metal-halogen exchange reaction (not shown in Scheme 3.1) was used to deactivate residual chloromethyl groups on the substrates and avoid cross-linking in the subsequent reaction steps. With the exception of the copolymer based on the core with $M_{\mathrm{w}} \approx 14000$ side chains, which had a polydispersity index $M_{\mathrm{w}} / M_{\mathrm{n}}=1.11$, all the other PS cores and copolymers had $M_{\mathrm{w}} / M_{\mathrm{n}} \leq 1.03$.

The characteristics of the PS cores and the corresponding copolymers are summarized in Table 3.1. The nomenclature used to identify the copolymers is based on composition and structure. For example, G1-30PS-HB-43 refers to an arborescent copolymer synthesized from a $G 1$ high branching functionality PS core with $M_{\mathrm{w}} \approx 30000$ side chains, and containing $43 \%$ PEO by weight. The fully extended length $\left(R_{\mathrm{PEO}}\right)$ for linear PEO chains with molecular weights identical to the PEO segments in the copolymers $\left(M_{\mathrm{PEO}}\right)$ were calculated as described 
Table 3.1. Characteristics of Arborescent PS-g-PEO Copolymers Used.

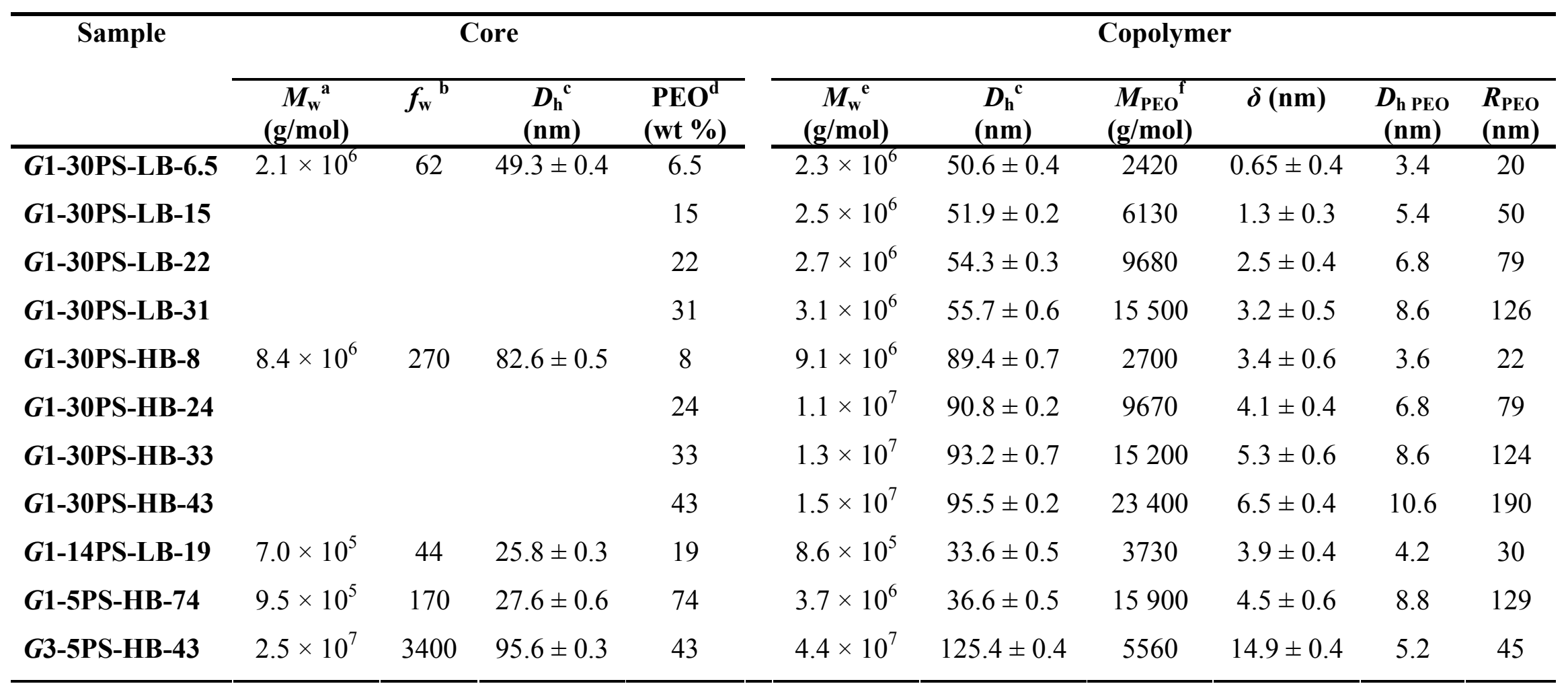

Absolute weight-average molecular weight from static light scattering measurements.

Branching functionality.

Hydrodynamic diameter from dynamic light scattering measurements. Uncertainties calculated as the standard deviation on 10 measurements.

PEO content based on FTIR analysis.

Calculated from core $M_{\mathrm{w}}$ and composition.

$\left[M_{\mathrm{w}}(\right.$ copolymer $)-M_{\mathrm{w}}($ core $\left.)\right] / f_{\mathrm{w}}(100 \%$ activation and initiation efficiency assumed $)$ 
previously. ${ }^{11 \mathrm{a}}$ The coiled hydrodynamic diameter in chloroform $\left(D_{\mathrm{h}} \mathrm{PEO}\right)$ was calculated from the equation

$$
D_{h}=2\left(3 M[\eta] / 5 \pi N_{A}\right)^{1 / 3}
$$

where $N_{\mathrm{A}}$ is Avogadro's number, $M$ is the molecular weight, and $[\eta]$ is the intrinsic viscosity of PEO in chloroform.

The low values obtained for the hydrodynamic thickness of the poly(ethylene oxide) shell, calculated as $\delta=1 / 2\left(D_{\mathrm{h}}\right.$ copolymer $-D_{\mathrm{h}}$ core $)$, clearly show that the PS- $g$-PEO arborescent micelles have a crew-cut structure, ${ }^{16}$ characterized by a large hydrophobic core surrounded by a thin hydrophilic corona. However unlike crew-cut micelles obtained from block copolymers with short hydrophilic segments, the PS-g-PEO arborescent crew-cut micelles are covalently bonded and hence have no critical micelle concentration (CMC). Comparison of the hydrodynamic PEO shell thickness $\delta$ among the samples, with the fully extended chain lengths, and with the coiled chain dimensions (Table 3.1) provides information about the conformation of the chains in the PEO shell. Thus while the molecular weight $\left(M_{\mathrm{PEO}}\right)$ of the PEO chains in some G1-30PS-LB and G1-30PS-HB samples is very similar, the shell thickness for the HB samples is significantly larger than for the LB samples. This is seen in comparing samples of similar compositions such as G1-30PS-LB-22 with G130PS-HB-24, and G1-30PS-LB-31 with G1-30PS-HB-33. It seems likely that because of increased steric interactions in the highly branched molecules, the PEO chains are slightly more extended in the HB samples than in the LB samples. The same reasoning also applies when comparing samples G1-5PS-HB-74 and G3-5PS-HB-43: The former has a thinner PEO shell in spite of its longer PEO chains. These results are in agreement with the work of de Gennes ${ }^{17}$ on the conformation of polymers grafted at interfaces. At low grafting densities the 
chains should adopt a coiled conformation in a good solvent, but start overlapping and be forced to stretch when the grafting density is increased. Comparison of the PEO shell thicknesses $(\delta)$ with the corresponding hydrodynamic values ( $\left.D_{\mathrm{h} \text { PEO }}\right)$ in the randomly coiled conformation and the fully extended chain lengths $R_{\mathrm{PEO}}$ (Table 3.1 ) shows that while the PEO chains may be slightly more extended in the HB sample series, they still essentially adopt a coiled conformation rather than an extended conformation: The $\delta$ values are much smaller than the fully extended chain lengths in all cases. The degree of extension of the PEO segments is low as compared to their fully extended chain length.

3.4.2 Topology of Langmuir-Blodgett Films. AFM pictures obtained for LangmuirBlodgett monolayers were used to study the influence of copolymer structure on the topology of the films. To minimize any contribution of compression to molecular assembly, the films were deposited without compression after spreading $100 \mu \mathrm{L}$ of 0.5
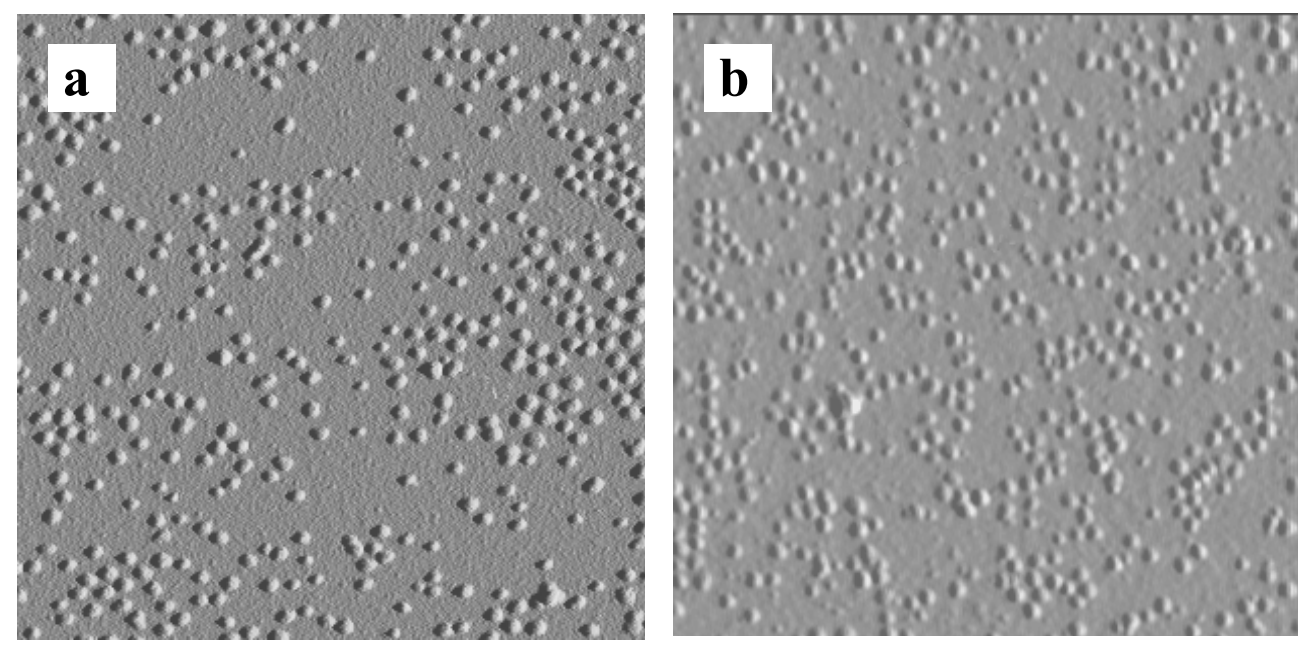

Figure 3.1. AFM amplitude images for copolymer G1-5PS-HB-74 deposited on (a) mica and (b) silicon. The images have a size of $1.5 \times 1.5 \mu \mathrm{m}^{2}$. 
$\mathrm{mg} / \mathrm{mL}$ copolymer solution onto the subphase. Apart from increasing the number of molecules present on the subphase, variations in the amount of solution spread within the $100-400 \mu \mathrm{L}$ range had no influence on film topology. Films transferred on cleaved mica and silicon wafer substrates were very similar (Figure 3.1), implying that substrate composition had no significant influence on film topology. For consistency, all the results presented subsequently were nonetheless obtained for films transferred on mica.

The discussion on the influence of copolymer structure and composition on film topology has been organized according to increasing core stiffness starting with the G130PS-LB sample series, followed by the G1-30PS-HB series, and ending with copolymers incorporating shorter PS side chains $\left(M_{\mathrm{w}} \approx 14000\right.$ and 5000).

3.4.2.1 G1-30PS-LB Samples. Film topology was greatly influenced by composition for copolymers incorporating a relatively low number of long, flexible PS segments (side chain $M_{\mathrm{w}} \approx 30000$ ) in the core. Height and amplitude AFM images for G1-30PS-LB samples $\left(f_{\mathrm{w}}=62\right)$ with different PEO contents are compared in Figure 3.2. The molecules of sample G1-30PS-LB-15 formed large spherical island-like aggregates with a broad size distribution and diameters reaching $200 \mathrm{~nm}$. Analysis of the film topology was carried out using the software (Nanoscope III 4.43r8) supplied with the AFM instrument. The average diameter of the few isolated molecules present was determined to be $55 \pm 6 \mathrm{~nm}$ by sectional analysis. Bearing analysis was used to analyze the distribution of molecular heights (film thickness) over the whole sample, yielding $16 \pm 3 \mathrm{~nm}$ for both isolated and aggregated molecules. Predominant clustering was also observed for sample G1-30PS-LB-6.5, the remaining isolated molecules having an average diameter of $46 \pm 5 \mathrm{~nm}$ and a comparable molecular height of $15 \pm 3 \mathrm{~nm}$. 

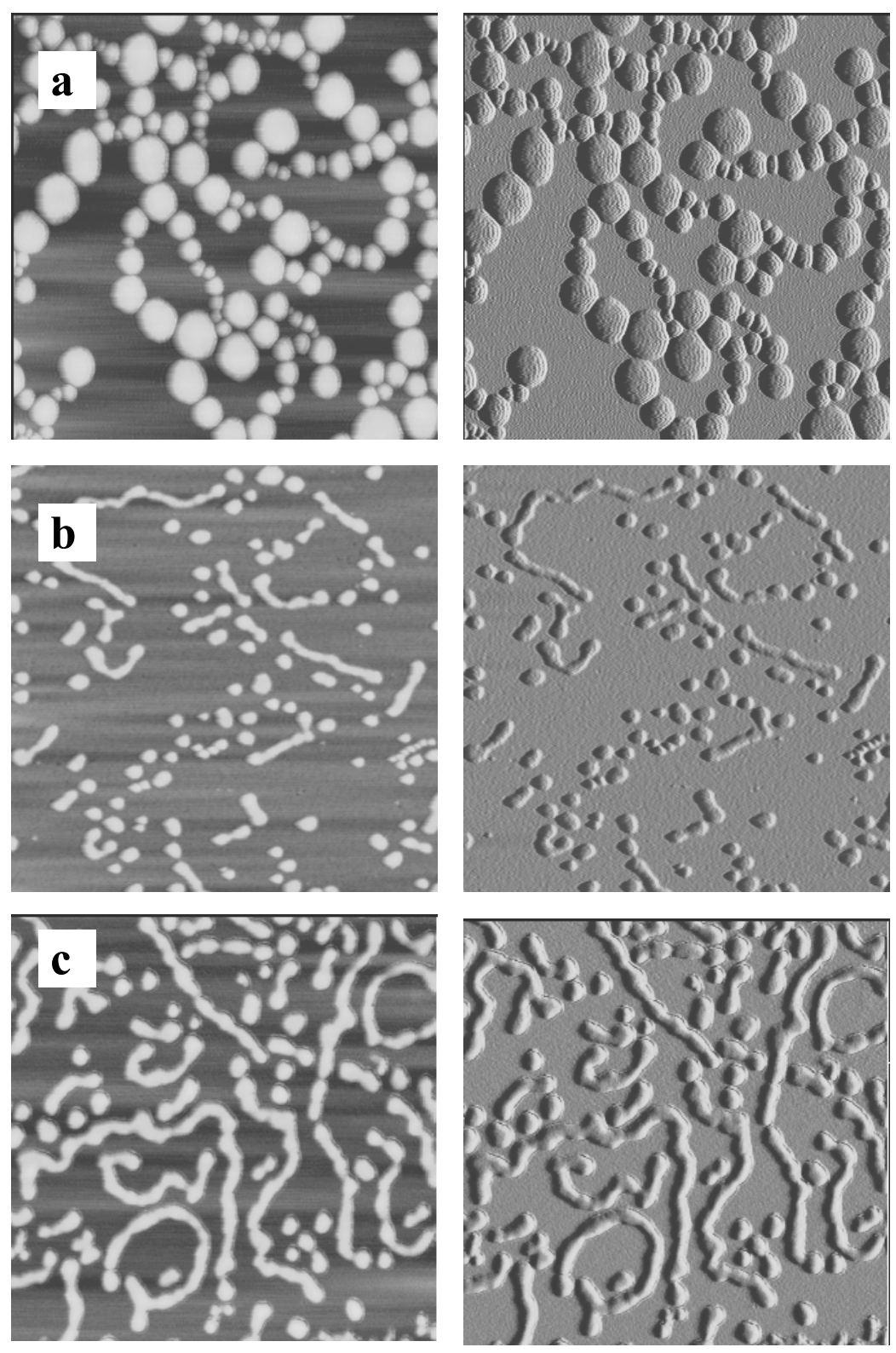

Figure 3.2. Height (left) and amplitude (right) images for Langmuir-Blodgett films for copolymers (a) G1-30PS-LB-15, (b) G1-30PS-LB-22, and (c) G1-30PS-LB-31. The images have a size of $1.5 \times 1.5 \mu \mathrm{m}^{2}$.

For a PEO content of $22 \%$, film topology changed from spherical aggregates to ribbon-like superstructures and isolated molecules. The AFM pictures for sample G1-30PS- 
LB-22 (Figure 3.2b) show that the Langmuir-Blodgett film contains both isolated molecules and ribbon-like structures extending over several molecules. Some ribbons have a length of over $300 \mathrm{~nm}$, while the width $(56 \pm 2 \mathrm{~nm})$ and thickness $(16 \pm 4 \mathrm{~nm})$ of the ribbons correspond to the dimensions of isolated molecules. The Langmuir-Blodgett film for sample G1-30PS-LB-31 (with 31\% PEO content by weight, Figure 3.2c) displays similar features, the ribbon structures being dominant and longer than in the last example.

The low thickness of the films as compared to the dimensions of isolated molecules shows that the copolymers adopt a flattened configuration at the air-water interface. The PEO content of the copolymers is obviously a major parameter influencing the topology of the films.

3.4.2.2 G1-30PS-HB Samples. The topology of Langmuir-Blodgett films prepared from the highly branched samples with long PS segments $\left(G 1-30 \mathrm{PS}-\mathrm{HB}, f_{\mathrm{w}}=270\right)$ is somewhat distinct from their low branching functionality $\left(G 1-30 \mathrm{PS}-\mathrm{LB}, f_{\mathrm{w}}=62\right)$ counterparts. Thus sample G1-30PS-HB-8, with a PEO content of $8 \%$ by weight, formed island-like clusters with a thickness of about $18 \pm 2 \mathrm{~nm}$ and a broad size distribution with diameters reaching over $500 \mathrm{~nm}$ (Figure 3.3a), in analogy to samples G1-30PS-LB-6.5 and G1-30PS-LB-15. Langmuir-Blodgett films obtained from G1-30PS-HB samples with higher PEO contents (24 and 43\%) are also provided in Figures 3.3b and 3.4c for comparison. In contrast to the copolymer with a low PEO content, these molecules either remained isolated or formed low-order aggregates and very few ribbon structures. The thickness of the individual molecules and the aggregates is about $20 \pm 3 \mathrm{~nm}$. The diameters of $100 \pm 5 \mathrm{~nm}$ and $120 \pm 5 \mathrm{~nm}$ determined for isolated molecules of G1-30PS-HB-24 and G1-30PS-HB-43, respectively, are comparable to the width of the aggregates. 
The fact that these copolymers are based on identical cores but have different diameters on the water surface suggests that the PEO chains are at least partly adsorbed
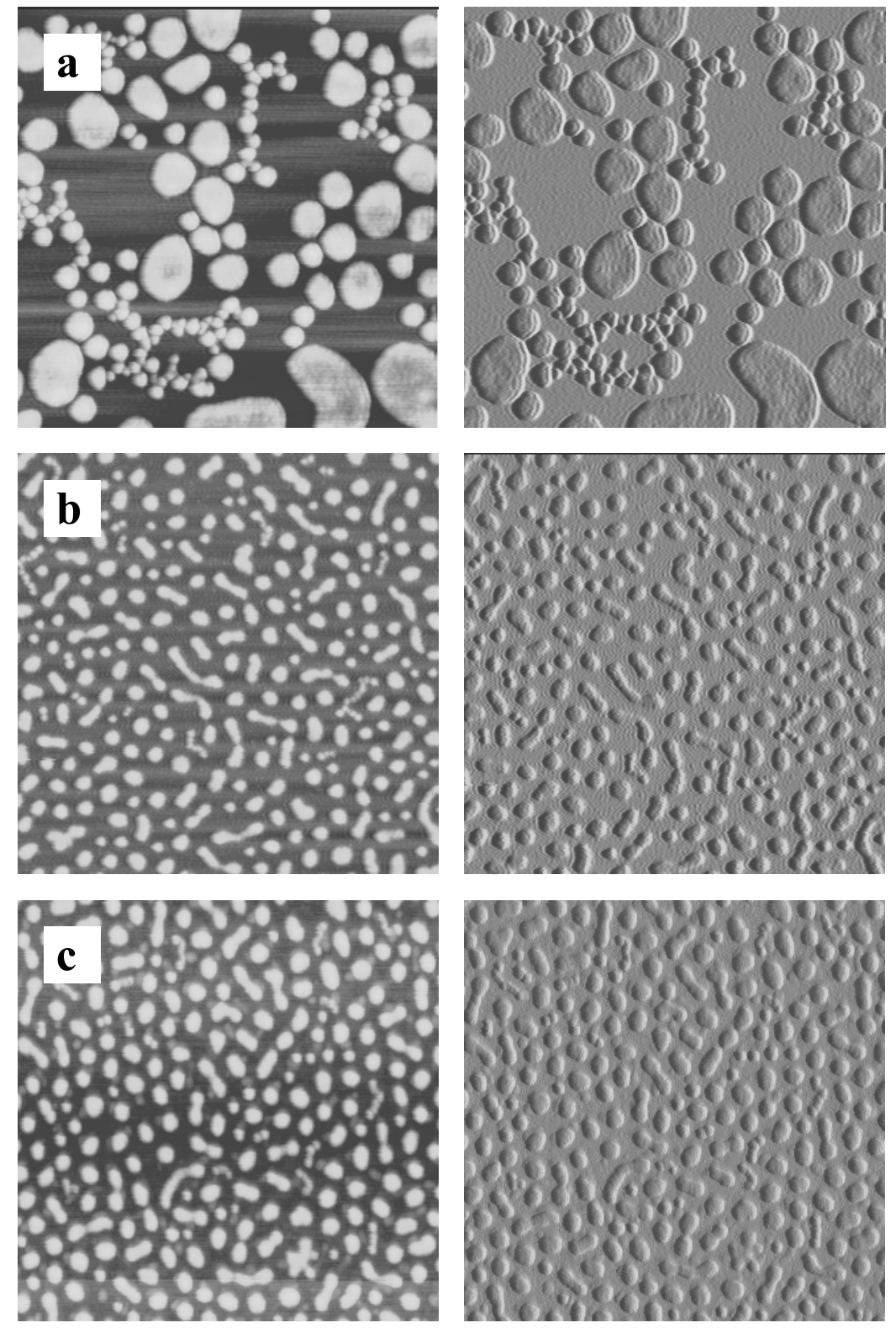

Figure 3.3. Height (left) and amplitude (right) AFM images of films for copolymers (a) G130PS-HB-8 (b) G1-30PS-HB-24, and (c) G1-30PS-HB-43. The images have a size of $1.5 \times$ $1.5 \mu \mathrm{m}^{2}$. 
on the water surface as suggested for PS-PEO block copolymers, ${ }^{8}$ star-block copolymers, ${ }^{9}$ and heteroarm (miktoarm) star ${ }^{10}$ copolymers. Langmuir-Blodgett films for sample G1-30PSHB-33 (not shown) had comparable features with isolated molecules and short ribbon-like structures, the isolated molecules having a diameter of $116 \pm 4 \mathrm{~nm}$ on the water surface, intermediate between G1-30PS-HB-24 and G1-30PS-HB-43.

The results presented so far show that while the self-assembly behavior of arborescent PS- $g$-PEO copolymers with long PS segments (side chain $M_{\mathrm{w}} \approx 30000$ ) in the core is dominated by their PEO content, increased core rigidity decreases the association level attained.

3.4.2.3 Other Samples. Sample G1-14PS-LB-19 has a core with $M_{\mathrm{w}}=14200$ polystyrene chains (approximately half in size of the chains in the G1-30PS-LB copolymers), and a branching functionality $f_{\mathrm{w}}=44$, somewhat lower than for the G1-30PS-LB samples. The main distinguishing characteristic of this sample is therefore its smaller core size $\left(D_{\mathrm{h}}=\right.$ $26 \mathrm{~nm}$, as compared to $49 \mathrm{~nm}$ for the G1-30PS-LB cores), since the PS side chains are still relatively long and flexible. Copolymer G1-14PS-LB-19 is a low branching functionality sample with a PEO content of $19 \%$ (i.e. slightly above the PEO content of sample G1-30PSLB-15, which displayed clustering). The Langmuir-Blodgett films obtained for this sample (Figure 3.4) contained isolated molecules, low-order aggregates, as well as many ribbon-like superstructures similar to those obtained for samples G1-30PS-LB-22 and G1-30PS-LB-31. The thickness and the width of the low-order aggregates and the ribbons were $7 \pm 2 \mathrm{~nm}$ and $75 \pm 3 \mathrm{~nm}$, respectively. The thickness is consistent with that of isolated molecules, but the width of the ribbons is roughly twice as large as for isolated molecules $(39 \pm 2 \mathrm{~nm})$. 
The fact that the film topology obtained for sample G1-14PS-LB-19 is similar to that of G1-30PS-LB-22 and G1-30PS-LB-31 in spite of the smaller size of its PS core suggests (on the basis of the limited information available) that the core size per se does not have much influence on film topology.
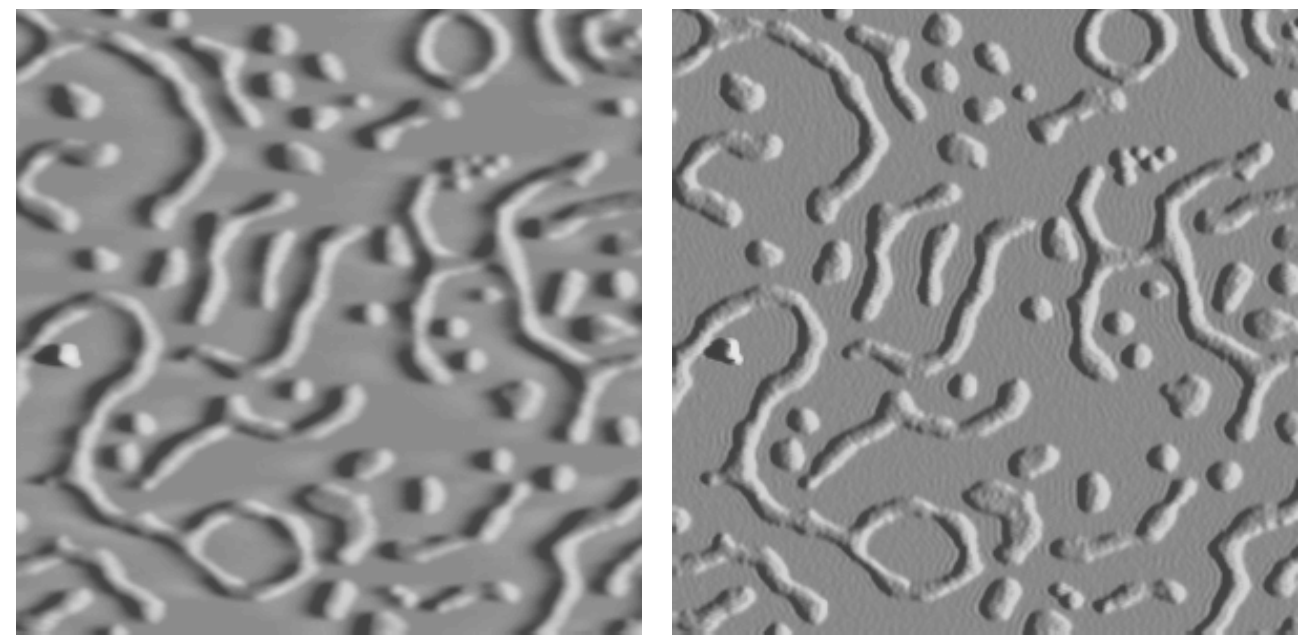

Figure 3.4. Height (left) and amplitude (right) AFM micrographs obtained for copolymer G1-15PS-HB-19. The images have a size of $1.5 \times 1.5 \mu \mathrm{m}^{2}$.

AFM height and amplitude images for a Langmuir-Blodgett film prepared from sample G1-5PS-HB-74 are provided in Figure 3.5. The molecules of this sample have a highly branched PS core $\left(f_{\mathrm{w}}=170\right)$ with very short $\left(M_{\mathrm{w}} \approx 5000\right)$ PS side chains and a high PEO content ( $74 \%$ by weight). The molecules are therefore significantly more rigid than in the previous examples but the core size $\left(D_{\mathrm{h}}=28 \mathrm{~nm}\right)$ is comparable to sample $G 1$-14PS-LB19, presumably due to stretching of the PS chains as a result of crowding. The hydrodynamic thickness of the PEO shell $(\delta)$ is also comparable to G1-14PS-LB-19 and to the samples in 
the G1-30PS-HB series with a high PEO content. The main distinguishing feature of these molecules with respect to sample G1-14PS-LB-19 is therefore their increased structural rigidity. The molecules of copolymer G1-5PS-HB-74 remained mostly isolated on the water surface, without formation of superstructures of the types observed for copolymers in the G130PS-LB and (to a lesser extent) the G1-30PS-HB series with high PEO contents. The average thickness of the molecules determined from bearing analysis of Figure 3.5 was $7 \pm 1$ $\mathrm{nm}$, while the average diameter derived from section analysis was $50 \pm 3 \mathrm{~nm}$.

Remembering that the G1-30PS-HB samples displayed a lower association level than the G1-30PS-LB samples, it appears that the enhanced rigidity of G1-5PS-HB-74 copolymer molecules may be an important factor contributing to their greater colloidal stability, although enhanced steric stabilization may also play a role since their PEO content is very high (74\%).
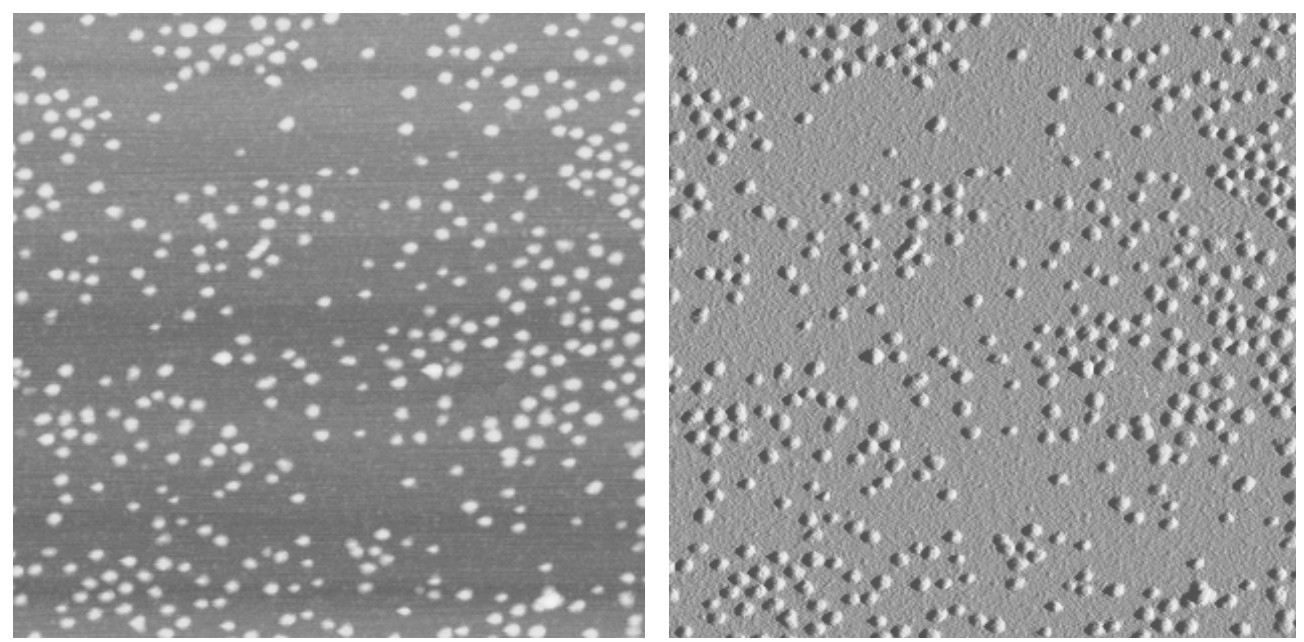

Figure 3.5. AFM height (left) and amplitude (right) images for copolymer G1-5PS-HB-74. The images have a size of $1.5 \times 1.5 \mu \mathrm{m}^{2}$. 
To investigate further the influence of molecular rigidity on the assembly process, the behavior of a copolymer with a much higher branching functionality (G3-5PS-HB-43, $f_{\mathrm{w}}=$ 3400) was also examined. This copolymer is characterized by a very rigid core structure, higher than for the G1-30PS-HB samples. The PEO content of G3-5PS-HB-43 is identical with G1-30PS-HB-43, the second largest sample studied in terms of hydrodynamic diameter of the core and the overall molecules. While the molecular weight of the PEO chains is much lower for the $G 3$ sample than for the $G 1$ sample, its hydrodynamic PEO shell thickness is significantly higher $\left(M_{\mathrm{PEO}}=5560, \delta=15 \mathrm{~nm}\right.$ and $M_{\mathrm{PEO}}=23400, \delta=6.5 \mathrm{~nm}$ for the $G 3$ and $G 1$ samples, respectively). The $G 3$ copolymer has the thickest PEO shell among all samples, albeit this is clearly the result of chain stretching rather than its modest PEO content ( $43 \%$ by weight).

The topology of the Langmuir-Blodgett film obtained for copolymer G3-5PS-HB-43 is characterized mainly by large clusters (Figure 3.6). Individual molecules with a diameter of $140 \pm 5 \mathrm{~nm}$ and a height of about $40 \pm 3 \mathrm{~nm}$ are clearly visible due to their very stiff structure, and are mainly arranged into a regular hexagonal packing array. This result is rather surprising, considering that sample G1-30PS-HB-43 (with an identical PEO content) displayed very little aggregation and that sample G1-5PS-HB-74 (with a small, rigid core) remained essentially non-associated.

Because the molecules of this sample incorporate a very dense $G 3$ PS core $\left(f_{\mathrm{w}}=\right.$ $3400)$ with short side chains $\left(M_{\mathrm{w}} \approx 5000\right)$, their behavior may approach that of rigid stabilized colloids or latex particles. ${ }^{18}$ The molecules form clusters analogous to poorly stabilized regular colloids, their dense structure hindering interpenetration. While the PEO shell of the copolymers has a high hydrodynamic thickness due to chain stretching, it is 
inefficient at hindering the aggregation of the molecules. The PEO chains on copolymer G35PS-HB-43 $\left(M_{\mathrm{PEO}}=5560\right)$ are intermediate in size between those of samples G1-30PS-LB$6.5\left(M_{\mathrm{PEO}}=2420\right)$ and G1-30PS-LB-15 $\left(M_{\mathrm{PEO}}=6130\right)$, and their stabilizing ability is likewise limited, as both copolymers also formed island-like clusters.
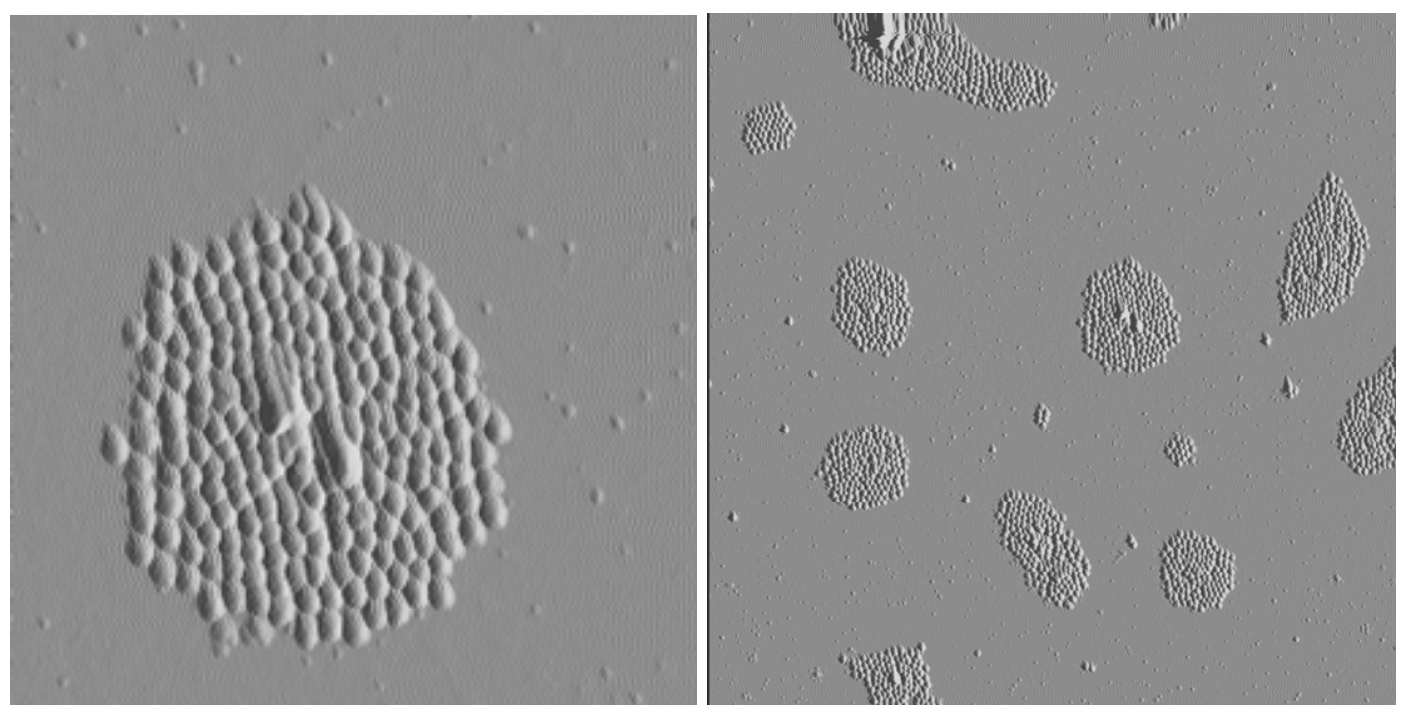

Figure 3.6. Amplitude AFM images for Langmuir-Blodgett films obtained from copolymer G3-5PS-HB-43, $2.5 \times 2.5 \mu \mathrm{m}^{2}$ (left) and $10 \times 10 \mu \mathrm{m}^{2}$ (right).

3.4.2.4 Comparison with Related Polymeric Amphiphiles. The results obtained demonstrate that arborescent PS- $g$-PEO copolymers associate spontaneously when spread at the air-water interface, and that the topology and the association level of the superstructures obtained both depend on the composition and the structure of the molecules. Comparisons with suitable polymeric amphiphiles related to the arborescent systems in terms of composition and/or structure would be useful to shed some light on the association mechanism leading to the formation of the superstructures observed. 
Conflicting explanations have been suggested for the aggregation mechanism of polymeric amphiphiles at the air-water interface, and in particular block copolymer amphiphiles. Some authors have reported the formation of supramolecular structures for PS$b$-PEO copolymers in chloroform solutions, and have therefore assumed their subsequent transfer to the air-water interface. ${ }^{19}$ In contrast, others suggested a compression-induced association mechanism on the basis of thermodynamic analysis ${ }^{20}$ and neutron reflectivity measurements. ${ }^{21}$ It is clear that monolayer compression is not an absolute requirement for superstructure formation, however. Thus linear PS- $b$-PEO copolymers ${ }^{22}$ and reverse starbranched PEO- $b$-PS copolymers (containing PS segments on the outer portion of the arms) ${ }^{23}$ both form superstructures of different topologies at the air-water interface in the absence of compression. The self-assembly of heteroarm (miktoarm) star-branched PEO- $b$ - $\mathrm{PS}_{3}{ }^{10}$ copolymers has also been reported, although the domains were irregular and not very distinct in that case.

Our own results are in agreement with the latter findings, as arborescent PS- $g$-PEO copolymer molecules spontaneously formed superstructures upon spreading at the air-water interface even in the absence of compression. Dynamic light scattering measurements for the arborescent PS-g-PEO copolymers did not reveal any aggregation in chloroform (Table 3.1), and the hydrodynamic diameters obtained are consistent with the dimensions determined from AFM measurements on isolated copolymer molecules. The absence of aggregated species in solution was further confirmed by CONTIN analysis of the correlation function $\mathrm{g}(\tau)$ obtained in the DLS experiments.

The topology of surface micelles formed by linear PS- $b$-PEO copolymers was found to be strongly composition-dependent. Thus PS- $b$-PEO copolymers with $18-24 \%$ PEO by 
weight yielded spherical aggregates of uniform dimensions at very low compression. ${ }^{22}$ When the PEO content was decreased to $7-9 \%$ by lowering the degree of polymerization of the PEO segment, a mixed topology with various types of ribbon-like structures was obtained. Mixtures of irregular spheres, spaghetti-shaped and ill-defined island-like clusters where also observed when a linear PS- $b$-PEO copolymer with 7\% PEO was compressed to a low surface pressure $(2 \mathrm{mN} / \mathrm{m}),{ }^{8 \mathrm{~h}}$ but only regular spheres where present for a copolymer with a PEO content of $92 \%$ by weight. ${ }^{8 f}$ Spherical and ribbon-like structures were likewise observed for Langmuir-Blodgett films obtained from three-arm reverse star PEO- $b$-PS copolymers with a PEO content of $20 \%$ by weight. ${ }^{23}$ The topology of films prepared from star-branched miktoarm PEO- $b$ - $\mathrm{PS}_{2}$ and $\mathrm{PEO}-b-\mathrm{PS}_{3}$ copolymers at the air-water interface was also examined and found to present similarities to their linear counterparts: ${ }^{10}$ At low surface pressures $(0.3 \mathrm{mN} / \mathrm{m})$ mixed morphologies with irregular spheres, short ribbon- and spaghetti-like structures, and island-like clusters were obtained for PEO contents of $11-13 \%$ by weight. The topology of the films changed to regular spherical and short ribbon-like aggregates when the PEO content was increased to $20-27 \%$ by weight ${ }^{10}$ by reducing the length of the PS segment in the copolymers.

The behavior of arborescent PS-g-PEO copolymers at the air-water interface clearly presents similarities to linear and star-block copolymers, as the film topology is strongly influenced by composition. Molecules with PEO contents in the $19-30 \%$ range tend to form ribbon-like structures irrespective of architecture, while either island-like clusters or complex morphologies are obtained at low PEO contents and spherical, non-associated species are dominant at high PEO contents. A major distinction for the arborescent PS-g-PEO copolymers is that topology is also influenced by the rigidity of the copolymer molecules, 
high branching functionality copolymers being generally characterized by a lower association level. The range of co-existing superstructures is also more limited for arborescent copolymers than for block and star-branched systems: Arborescent PS-g-PEO copolymers with a very compact PS core (G1-5PS-HB) remained as isolated spheres at the air-water interface, while 'crew-cut' copolymers with a PEO content of less than or equal to $15 \%$ by weight or incorporating a very rigid (G3-5PS-HB) core formed only island-like clusters. Combinations of ribbon-like superstructures and low-order aggregates were observed for copolymers with intermediate PEO contents ( $22-43 \%$ by weight), the length of the ribbons (degree of association) being influenced by the rigidity of the arborescent PS core.

3.4.2.5 Association Mechanism. It has been demonstrated that arborescent polystyrene molecules behave like flexible colloidal particles with a diffuse and dynamically rough surface. ${ }^{24}$ Arborescent PS-g-PEO unimolecular micelles are hence expected to display properties comparable to sterically stabilized colloids. The interactions between such molecules should depend not only upon interfacial forces, but also on the ability of the molecules to deform and overlap on their surfaces. ${ }^{24}$ The free molecules at the air-water interface are not static but rather randomly moving due to Brownian motions. ${ }^{25}$ Therefore to prevent coalescence of the molecules, a balance must exist for the van der Waals attractive forces between adjacent PS cores and the repulsive steric interactions between the coronas due to the elastic deformation of the PEO chains. Even in the absence of compression, deformation of the molecules can occur due to unfavorable interactions between the PS and PEO chains, and the interactions of each component with the water subphase. Interactions of this type have been associated with phase separation in block copolymers leading to spontaneous association at the air-water interface. ${ }^{22,26}$ For low PEO content samples van der 
Waals interactions between the PS cores are expected to be more significant, which explains the observed coalescence of the molecules into large island-like clusters. For higher PEO content samples, the degree of deformation of the molecules should become an important factor in the association process. While repulsive forces may suffice to prevent coalescence, elastic deformation of the PEO chains taking place as the molecules move randomly on the water subphase may lead to significant van der Waals attraction forces between cores, inducing the formation of superstructures. The G1-30PS-HB samples are expected to be more rigid $\left(f_{\mathrm{w}}=270\right)$ than the $G 1-30 \mathrm{PS}-\mathrm{LB}$ samples $\left(f_{\mathrm{w}}=62\right)$. The deformability of the PEO chains for the HB samples is presumably lower than for the LB samples, leading to the lower degree of association observed. The association level for the low branching functionality samples is conversely expected to be higher, due to the greater ease of elastic deformation of the PEO chains. An intriguing aspect of the self-assembly of samples with intermediate PEO contents is the preferred formation of ribbon-like superstructures at the expense of island-like clusters. This could be due to an increase in the lateral PEO chain density of the surface micelle as a result of association. A semi brush-like conformation for the lateral chains would disfavor association from the sides.

\subsection{Conclusions}

It has been shown that arborescent PS-g-PEO copolymers form stable monolayers and tend to associate spontaneously at the air-water interface even without compression. By varying the composition and the structure of arborescent PS- $g$-PEO copolymers, the association behavior of the amphiphiles could be tailored to favor the formation of either 
ribbon-like superstructures of uniform width, large island-like clusters with a wide size distribution, or non-associated spherical micelles of uniform size.

\subsection{Acknowledgements}

We acknowledge the financial support of the Natural Sciences and Engineering Research Council of Canada (NSERC) and the International Council for Canadian Studies (ICCS). We also extend our gratitude to Prof. S. Mikkelsen for providing access to the atomic force microscopy equipment.

\subsection{References}

1. (a) Zeng, F.; Zimmerman, S. C. Chem. Rev. 1997, 97, 1681. (b) Tsukruk, V. V. Adv. Mater. 1998, 10, 253. (c) Emrick, T.; Fréchet, J. M. J. Curr. Opin. Colloid Interface Sci. 1999, 4, 15. (d) Dykes, G. M. J. Chem. Technol. Biotechnol. 2001, 76, 903. (e) Tully, D. C.; Fréchet, J. M. J. Chem. Commun. 2001, 1229.

2. (a) Tomalia, D. A.; Brothers II, H. M.; Piehler, L. T.; Durst, H. D.; Swanson, D. R. Proc. Natl. Acad. Sci. USA 2002, 99, 5081. (b) Betley, T. A.; Hessler, J. A.; Mecke, A.; Holl, M. M. B.; Orr, B. G.; Uppuluri, S.; Tomalia, D. A.; Baker Jr., J. R. Langmuir 2002, $18,3127$.

3. Zimmerman, S. C.; Zeng, F.; Reichert, D. E. C.; Kolotuchin, S. V. Science 1996, 271, 1095.

4. (a) Huck, W. T. S.; van Veggel, F. C. J. M.; Kropman, B. L.; Blank, D. H. A.; Keim, E. G.; Smithers, M. M. A.; Reinhoudt, D. N. J. Am. Chem. Soc. 1995, 117 8293. (b) Andres, P. R.; Schubert, U. S. Adv. Mater. 2004, 16, 1043. 
5. Schenning, A. P. H. J.; Elissen-Román, C.; Weener, J.-W.; Baars, M. W. P. L.; van der Gaast, S. J.; Meijer, E. W. J. Am. Chem. Soc. 1998, 120, 8199.

6. (a) Baker, L. A.; Zamborini, F. P.; Sun, L.; Crooks, R. M. Anal. Chem. 1999, 71, 4403.

(b) Zhang, X.; Wilhelm, M.; Klein, J.; Pfaadt, M.; Meijer, E. M. Langmuir 2000, 16, 3884.

7. (a) Sheiko, S. S.; Muzafarov, A. M.; Winkler, R. G.; Getmanova, E. V.; Eckert, G.; Reineker, P. Langmuir 1997, 13, 4172. (b) Tsukruk, V. V.; Rinderspacher, F.; Bliznyuk, V. N. Langmuir 1997, 13, 2171. (c) Tokuhisa, H.; Zhao, M.; Baker, L. A.; Phan. V. T.; Dermody, D. L.; Garcia, M. E.; Peez, R. F.; Crooks, R. M.; Mayer, T. T. J. Am. Chem. Soc. 1998, 120, 4492. (d) Xu, F. T.; Street, S. C.; Barnard, J. A. Langmuir 2003, 19, 3066 .

8. (a) Bijsterbosch, H. D.; de Haan, V. O.; de Graaf, A. W.; Mellema, M.; Leermakers, F. A. M.; Cohen Stuart, M. A.; van Well, A. A. Langmuir 1995, 11, 4467. (b) Fauré, M. C.; Bassereau, P.; Carignano, M. A.; Szleifer, I.; Gallot, Y.; Andelman, D. Eur. Phys. J. B. 1998, 3, 365. (c) Rother, G.; Findenegg, G. H. Colloid Polym. Sci. 1998, 276, 496. (d) Gragson, D. E.; Jensen, J. M.; Baker, S. M. Langmuir 1999, 15, 6127. (e) Cox, J. L.; Yu, K.; Eisenberg, A.; Lennox, R. B. Phys. Chem. Chem. Phys. 1999, 1, 4417. (f) Baker, S. M.; Leach, K. A.; Devereaux, C. E.; Gragson, D. E. Macromolecules 2000, 33, 5432. (g) Akentiev, A. V.; Miller, R.; Noskov, B. A. Colloid J. 2002, 64, 653. (h) Devereaux, C. A.; Baker, S. M. Macromolecules 2002 35, 1921. (i) Rivillon, S.; Muñoz, M. G.; Monroy, F.; Ortega, F.; Rubio, R. G. Macromolecules 2003, 36, 4068.

9. Francis, R.; Taton, D.; Logan, J. L.; Masse, P.; Gnanou, Y.; Duran, R. S. Macromolecules 2003, 36, 8253 . 
10. Peleshanko, S.; Jeong, J.; Gunawidjaja, R.; Tsukruk, V. V. Macromolecules 2004, 37, 6511.

11. (a) Gauthier, M.; Tichagwa, L.; Downey, J. S.; Gao S. Macromolecules 1996, $29,519$.

(b) Cao, L. M.Sc. Thesis, University of Waterloo, Waterloo, Ontario, 1997.

12. Iyver, J.; Hammond, P. T. Langmuir 1999, 15, 1299.

13. Nierengarten, J.-F.; Eckert, J.-F.; Rio, Y.; del Pilar Carreon, M.; Gallani, J.-L.; Guillon, D. J. Am. Chem. Soc. 2001, 123, 9743.

14. Tanaka, K.; Dai, S.; Kajiyama, T; Aoi, K.; Okada, M. Langmuir 2003, 19, 1196.

15. Teertstra, S. J.; Gauthier, M. Prog. Polym. Sci. 2004, 29, 277.

16. Zhang, L.; Eisenberg, A. J. Am. Chem. Soc. 1996, 118, 3168.

17. de Gennes, P. G. Macromolecules 1980, 13, 1069.

18. (a) Aveyard, R.; Clint, J. H.; Nees, D.; Paunov, V. N. Langmuir 2000, 16, 1969. (b) Raşa, M.; Kuipers, B. W. M.; Philipse, A. P. J. Colloids Interface Sci. 2002, 250, 303.

19. Gonçalves da Silva, A. M.; Simões Gamboa, A. L.; Martinho, J. M. G. Langmuir 1998, 14,5327 .

20. Israelachvili, J. Langmuir 1994, 10, 3774.

21. An, S. W.; Su, T. J.; Thomas, R. K.; Baines, F. L.; Billingham, N. C.; Armes, S. P.; Penfold, P. J. Phys. Chem. B 1998, 102, 387.

22. Cox, J. K.; Yu, K.; Constantine, B.; Eisenberg, A.; Lennox, R. B. Langmuir 1999, 15, 7714.

23. Francis, R.; Skolnik, A. M.; Carino, S. R.; Logan, J. L.; Underhill, R. S.; Angot, S.; Taton, D.; Gnanou, Y.; Duran, R. S. Macromolecules 2002, 35, 6485.

24. Sheiko, S. S.; Gauthier, M.; Möller, M. Macromolecules 1997, 30, 2343. 
25. Hu, Y.; Lee, K. Y. C.; Israelachvili, J. Langmuir 2003, 19, 100.

26. Kumaki, J.; Hashimoto, T. J. Am. Chem. Soc. 1998, 120, 423. 


\section{Chapter 4}

\section{Induced Association of Arborescent Polystyrene-graft-}

Poly(ethylene oxide) Copolymers at the Air-water

Interface 


\subsection{Abstract}

The influence of compression, subphase temperature, and solution aging on the association behavior of arborescent polystyrene-graft-poly(ethylene oxide) (PS-g-PEO) copolymers at the air-water interface was investigated using the Langmuir balance and atomic force microscopy (AFM) techniques. These dendritic molecules are able to form stable condensed monolayers with surface compressional moduli greater than $250 \mathrm{mN} / \mathrm{m}$. The variations in film thickness observed as a function of surface pressure suggest that at low surface pressures (gas-like phase), the PEO chains remain adsorbed at the air-water interface. At higher surface pressures (condensed phase of the isotherm), the PEO chains gradually desorb into the subphase and adopt a more brush-like conformation. Large island-like clusters with a broad size distribution were observed for samples with PEO contents up to $15 \%$ by weight. In contrast, copolymers with PEO contents of $22-43 \%$ displayed enhanced association into ribbon-like superstructures upon compression. The same effect was observed even in the absence of compression when the temperature of the subphase was increased or when the solutions were aged. The enhanced association was attributed to increased van der Waals attractive forces between the PS cores relative to the steric repulsive forces between PEO chains in the coronas, due to decreased solubility of the PEO segments at higher temperatures and to photodegradation of the PEO segments upon aging. It was shown that free polymer contaminant may also play a role in bridging adjacent molecules, but does not suffice to explain enhanced ribbon formation. 


\subsection{Introduction}

Amphiphilic copolymers are an interesting class of materials capable of forming welldefined micellar superstructures of different topologies. ${ }^{1}$ This characteristic makes amphiphilic copolymers useful in applications such as colloid stabilization, solubilization, controlled release, lubrication, and microlithography. ${ }^{2}$ Amphiphilic block copolymer and surfactant micelles, sometimes referred to as association colloids, have distinct characteristics from conventional colloidal particles. ${ }^{3}$ Unlike rigid colloidal particles held by strong binding forces such as covalent or ionic bonds, micellar systems based on amphiphilic block copolymers and surfactant molecules result from weak non-covalent forces. A change in their immediate environment may affect not only the interactions between the colloidal particles but also intraparticle forces, which may lead to modifications in their size and shape. ${ }^{3}$

Amphiphilic dendritic copolymers are distinct due to their covalently bonded structure allowing them to retain their overall shape irrespective of their immediate environment. They can be obtained by derivatizing a dendritic core with small molecules or polymer segments of a different composition. ${ }^{4}$ Dendritic polymers can be regarded as soft colloids, and differ from regular colloidal particles by their variable density distribution and dynamic surface. ${ }^{5}$ In contrast to linear copolymer micelles, amphiphilic dendritic micelles can be considered as stabilized colloids and are expected to exhibit peculiar interfacial properties due to conformational constraints brought about by their high degree of branching. ${ }^{5}$

Arborescent polystyrene-graft-poly(ethylene oxide) (PS-g-PEO) copolymers are dendritic amphiphiles incorporating a highly branched PS core surrounded by flexible PEO chains. ${ }^{6}$ These molecules can be regarded as sterically stabilized soft colloids, and were 
shown in the previous Chapter to spontaneously self-assemble at the air-water interface even without compression. The influence of copolymer structure and composition on the association behavior of these molecules in Langmuir and Langmuir-Blodgett monolayers was investigated. In the current study, the focus is set on the influence of external stimuli on monolayer formation for selected arborescent PS-g-PEO copolymers. Changes in monolayer topology resulting from compression, subphase temperature variations, and gradual degradation of the stabilizing PEO layer under UV irradiation of the copolymer solutions are reported. Samples with different branching densities were selected to study the influence of conformational constraints on the assembly process in the presence of these external stimuli.

\subsection{Experimental Section}

The arborescent PS-g-PEO copolymers used in this study were synthesized by anionic grafting of PEO chains from $G 1$ arborescent polystyrene cores terminally functionalized with hydroxyl groups. ${ }^{6}$ All the copolymers used had narrow molecular weight distributions $\left(M_{\mathrm{w}} / M_{\mathrm{n}} \leq 1.03\right)$. To prepare a Langmuir monolayer, a $0.5 \mathrm{mg} / \mathrm{mL}$ copolymer solution in chloroform (Merck, HPLC grade) was sonicated for $30 \mathrm{~min}$, and $100 \mu \mathrm{L}$ of the solution was spread on the Milli-Q water (18 M $2 . \mathrm{cm})$ subphase in a custom-built $43 \times 15$ $\mathrm{cm}^{2}$ Langmuir balance trough. Compression was started after 15 min (to allow for solvent evaporation), at a rate of $10 \mathrm{~mm} / \mathrm{min}$. Dynamic light scattering measurements were used to estimate the hydrodynamic diameter of the core-shell copolymers in chloroform before and after aging, and to confirm complete sample dissolution before the spreading experiments. To transfer the monolayers onto solid substrates for the production of Langmuir-Blodgett films, freshly cleaved mica $\left(\approx 1 \times 1 \mathrm{~cm}^{2}\right)$ was first immersed into the subphase before the solutions 
were spread. After compression to the desired surface pressure $(\pi)$, the substrate was pulled through the interface at a rate of $3 \mathrm{~mm} / \mathrm{min}$ using a Lauda FL-1 Filmlift. To study the influence of temperature on the Langmuir and Langmuir-Blodgett films, water was circulated in the base of the Langmuir balance trough using a circulating temperature controller. For the UV irradiation experiments, low pressure mercury lamps (Rayonet Photochemical Reactor Lamp, No. RPR-2537A) were used at a distance of $50 \mathrm{~cm}$ from the sample solutions. The topology of the Langmuir-Blodgett films obtained was investigated with atomic force microscopy on a Nanoscope III Scanning Probe Microscope (Digital Instrument Inc., Santa Barbara, CA) operating in the tapping mode using NanoProbe ${ }^{\mathrm{TM}}$-TESP silicon cantilever tips having a resonant frequency between $260-410 \mathrm{kHz}$. Differential scanning calorimetry (DSC) measurements were done on a Q 100 instrument from TA Instruments. The samples $(5-10 \mathrm{mg})$ were sealed in aluminum pans, and the cooling and heating rates were set at 10 ${ }^{\circ} \mathrm{C} / \mathrm{min}$ for the measurements.

\subsection{Results and Discussion}

The characteristics of the copolymers investigated are summarized in Table 4.1. The nomenclature used for the samples is based on both composition and structure. Most copolymers incorporated $G 1$ cores of either low $\left(\mathrm{LB}, f_{\mathrm{w}}=62\right)$ or high $\left(\mathrm{HB}, f_{\mathrm{w}}=270\right)$ branching functionalities obtained by grafting linear PS side chains $\left(M_{\mathrm{w}} \approx 30000\right)$ onto randomly chloromethylated $G 0$ (side chain $M_{\mathrm{w}} \approx 30000$ ) backbone. The PEO content of the copolymers ranged from 6.5 to $43 \%$ by weight. Sample G3-5PS-HB-43 was constructed from much shorter $\left(M_{\mathrm{w}} \approx 5000\right)$ side chains, had a very high branching functionality $\left(f_{\mathrm{w}}=3400\right)$, and a PEO content of $43 \%$ by weight. The branching functionality $f_{\mathrm{w}}$, corresponding to the 
number of side chains added in the last generation (and the number of PEO chains present), was calculated as discussed in the previous Chapter.

4.4.1 Differential Scanning Calorimetry. DSC measurements were used to estimate the degree of crystallinity and the melting temperature of the PEO segments, and the glass transition temperature $\left(T_{\mathrm{g}}\right)$ of the PS cores and the copolymers. The emphasis was set on samples with longer PS segments (core side chain $M_{\mathrm{w}} \approx 30000$ ) to investigate the influence of branching functionality on $T_{\mathrm{g}}$ and crystallinity. The degree of crystallinity $(\alpha)$ was calculated $^{7}$ as the ratio of the measured heat of fusion per gram of PEO in the sample to the standard heat of fusion for $100 \%$ crystalline PEO, $\Delta \mathrm{H}_{\mathrm{f}}=205 \mathrm{~J} / \mathrm{g}{ }^{8}$

The degree of crystallinity of the PEO chains in the G1-30PS samples is higher for HB than for LB copolymers with comparable PEO contents (Table 4.2), and correlates to some extent with the PEO shell thickness, $\delta$ (Table 4.1). Samples with larger $\delta$ values tend to have higher degrees of crystallinity, although the melting temperature remained relatively constant $\left(T_{\mathrm{m}}=56 \pm 3{ }^{\circ} \mathrm{C}\right)$ for all samples with a detectable crystallinity. The DSC measurements were also carried out with Milli-Q water added in a 1:1 weight ratio to the samples, to simulate the conditions experienced by the copolymers on the water subphase, since extensive chain crystallization could be a significant driving force in the assembly process. This possibility should not be a concern here, as the degree of crystallinity was greatly reduced under these conditions (Table 4.2). The G1-30PS-LB, G1-30PS-HB, and the G3-5PS-HB PS cores displayed sharp glass transitions at $104.7 \pm 0.4{ }^{\circ} \mathrm{C}$ (Figure 4.1 inset). The transitions observed for the G1-30PS-LB and G1-30PS-HB copolymers where much broader however, suggesting partial mixing of the branched polystyrene core with the flexible PEO chains. ${ }^{9}$ The $T_{\mathrm{g}}$ values for the $G 1-30 \mathrm{PS}-\mathrm{LB}$ and $G 1-30 \mathrm{PS}-\mathrm{HB}$ copolymers (80 
Table 4.1. Characteristics of Arborescent Copolymers Used.

\begin{tabular}{|c|c|c|c|c|c|c|c|c|}
\hline \multicolumn{5}{|c|}{ Core } & \multicolumn{4}{|c|}{ Copolymer } \\
\hline Sample & $\begin{array}{c}M_{\mathrm{w}}^{\mathrm{a}} \\
(\mathrm{g} / \mathrm{mol})\end{array}$ & $f_{\mathrm{w}}$ & $\begin{array}{c}D_{\mathrm{h}}^{\mathrm{b}} \\
(\mathrm{nm})\end{array}$ & $\begin{array}{c}\text { PEO }^{c} \\
(\text { wt } \%)\end{array}$ & $\begin{array}{c}M_{\mathrm{w}}^{\mathrm{d}} \\
(\mathrm{g} / \mathrm{mol})\end{array}$ & $\begin{array}{c}D_{\mathrm{h}}^{\mathrm{b}} \\
(\mathrm{nm})\end{array}$ & $\begin{array}{c}M_{\text {PEO }}{ }^{\mathrm{e}} \\
(\mathrm{g} / \mathrm{mol})\end{array}$ & $\delta^{\mathrm{f}}(\mathrm{nm})$ \\
\hline G1-30PS-LB-6.5 & $2.1 \times 10^{6}$ & 62 & $49.3 \pm 0.4$ & 6.5 & $2.3 \times 10^{6}$ & $50.6 \pm 0.4$ & 2420 & $0.65 \pm 0.04$ \\
\hline G1-30PS-LB-15 & & & & 15 & $2.5 \times 10^{6}$ & $51.9 \pm 0.2$ & 6130 & $1.3 \pm 0.3$ \\
\hline G1-30PS-LB-22 & & & & 22 & $2.7 \times 10^{6}$ & $54.3 \pm 0.3$ & 9680 & $2.5 \pm 0.4$ \\
\hline G1-30PS-LB-31 & & & & 31 & $3.1 \times 10^{6}$ & $55.7 \pm 0.6$ & 15500 & $3.2 \pm 0.5$ \\
\hline G1-30PS-HB-8 & $8.4 \times 10^{6}$ & 270 & $82.6 \pm 0.5$ & 8 & $9.1 \times 10^{6}$ & $89.4 \pm 0.7$ & 2700 & $3.4 \pm 0.6$ \\
\hline G1-30PS-HB-24 & & & & 24 & $1.1 \times 10^{7}$ & $90.8 \pm 0.2$ & 9670 & $4.1 \pm 0.4$ \\
\hline G1-30PS-HB-33 & & & & 33 & $1.3 \times 10^{7}$ & $93.2 \pm 0.7$ & 15200 & $5.3 \pm 0.6$ \\
\hline G1-30PS-HB-43 & & & & 43 & $1.5 \times 10^{7}$ & $95.5 \pm 0.2$ & 23400 & $6.5 \pm 0.4$ \\
\hline G3-5PS-HB-43 & $2.5 \times 10^{7}$ & 3400 & $95.6 \pm 0.3$ & 43 & $4.4 \times 10^{7}$ & $125.4 \pm 0.4$ & 5560 & $14.9 \pm 0.4$ \\
\hline
\end{tabular}

Absolute weight-average molecular weight from static light scattering measurements

Hydrodynamic diameter from DLS measurements. Uncertainties calculated as the standard deviation on 10 measurements Composition based on FT-IR analysis

Calculated from core $M_{\mathrm{w}}$ and composition

Molecular weight of PEO chains, $\left[M_{\mathrm{w}}(\right.$ copolymer $)-M_{\mathrm{w}}($ core $\left.)\right] / f_{\mathrm{w}}$

Hydrodynamic thickness of PEO shell, $\delta=1 / 2\left(D_{\mathrm{h}}\right.$ copolymer $-D_{\mathrm{h}}$ core $)$ 
Table 4.2. Crystallinity of PEO and Phase Transition Temperatures for PS- $g$-PEO Copolymers.

\begin{tabular}{cccc}
\hline Sample & $\boldsymbol{\alpha}(\%)$ & $\begin{array}{c}\boldsymbol{\alpha}(\%) \\
(\text { polymer/water })\end{array}$ & $\boldsymbol{T}_{\mathrm{g}}(\mathrm{PS})$ \\
\hline $\boldsymbol{G 1 - 3 0 P S - L B - 1 5}$ & 0 & 0 & 93 \\
G1-30PS-LB-22 & 4.5 & 0 & 90 \\
G1-30PS-LB-31 & 1.7 & 0 & 91 \\
G1-30PS-HB-8 & 0.6 & 0 & 80 \\
G1-30PS-HB-24 & 12 & 1.0 & 90 \\
G1-30PS-HB-33 & 11 & 0.7 & 92 \\
G1-30PS-HB-43 & 13 & 1.5 & 90 \\
G3-5PS-HB-43 & 57 & 6.2 & 99 \\
\hline
\end{tabular}

$-93{ }^{\circ} \mathrm{C}$ ) were significantly lower than $105{ }^{\circ} \mathrm{C}$, but no clear trend was observed in terms of the influence of copolymer structure and composition on $T_{\mathrm{g}}$.

The existence of distinct thermal transitions for the PS core and the PEO chains is consistent with a phase-separated morphology, in analogy to other polymer systems with a core-shell morphology such as polystyrene-poly(butyl acrylate) core-shell latex particles, ${ }^{10}$ alkyl-terminated hyperbranched polyesters, ${ }^{11}$ and to phase-separated block copolymers in general. The higher $T_{\mathrm{g}}\left(99^{\circ} \mathrm{C}\right)$ and degree of crystallinity $(57 \%)$ of the PEO chains observed for sample G3-5PS-HB-43, $\left(f_{\mathrm{w}}=3400\right)$ suggests better phase separation in that sample. 


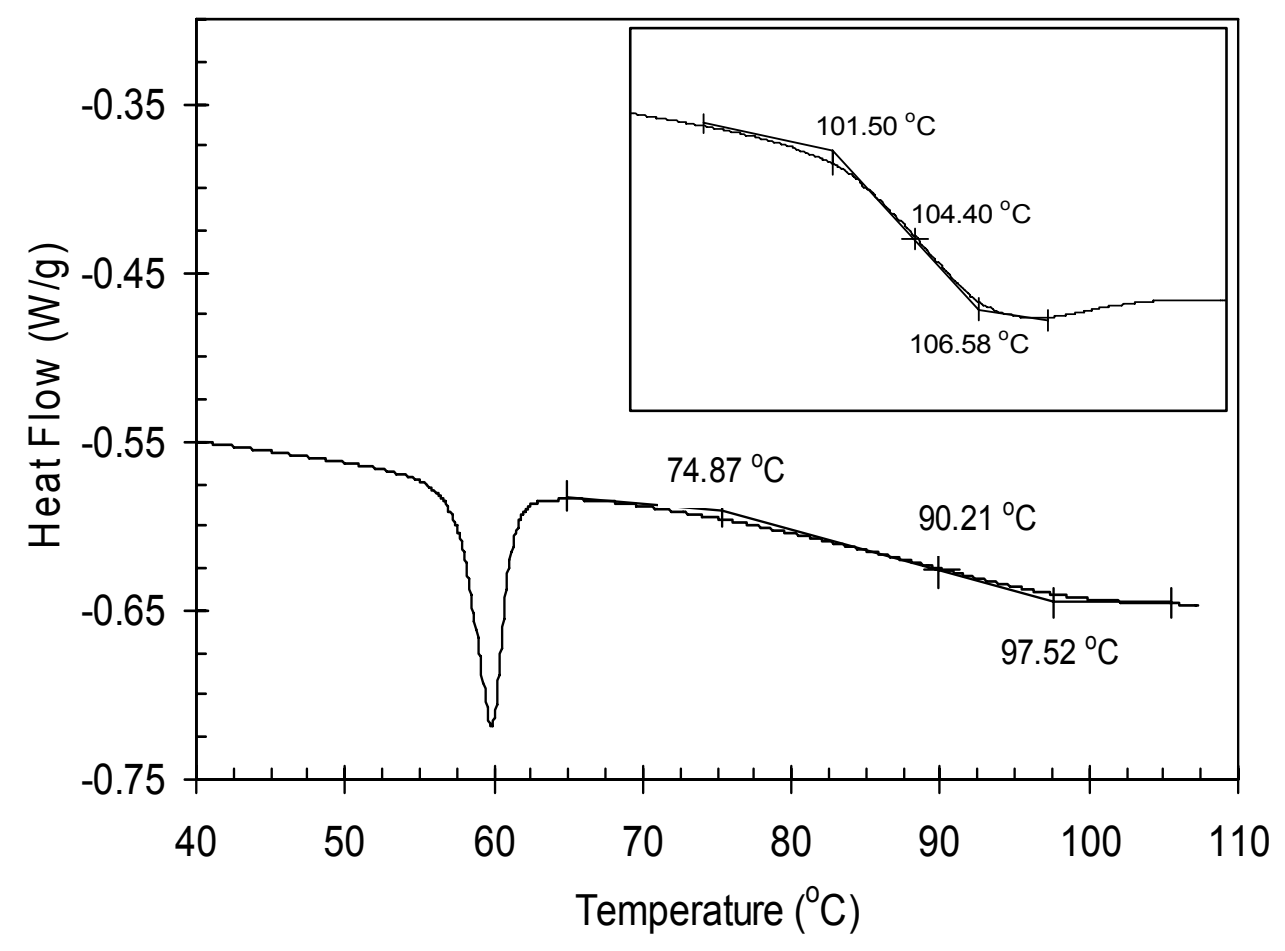

Figure 4.1. DSC thermograms comparing the broad glass transition observed for copolymer G1-30PS-LB-22 with the parent polystyrene core (G1-30PS-LB).

4.4.2 Langmuir Monolayers. The surface pressure-area ( $\pi-A)$ compression isotherms obtained for the copolymers were found to be independent of compression rate within $10-$ $30 \mathrm{~mm} / \mathrm{min}$ range. All copolymers displayed the characteristic gaseous-expanded, liquidcondensed, and solid-condensed phases typical of stable monolayers, ${ }^{12}$ except the G1-5PSHB-43 copolymer which showed characteristics of unstable monolayers. While the transitions observed do not correspond to real 'phase' transitions, they should reflect changes 
in the structure of the monolayer. The isotherms for both low and high branching functionality $G 1$ samples were fully reversible upon compression and decompression up to the end of the gaseous-expanded phase of the monolayers, as no significant hysteresis was observed. However, irreversible isotherms were obtained when the monolayers were compressed to the solid-condensed phase and decompressed. The isotherm for sample G130PS-LB-31, provided in Figure 4.2d, is typical and has a gaseous-expanded phase extending from surface pressures $\pi=0-6 \mathrm{mN} / \mathrm{m}$. The molecules are far apart in this region, the

interactions between the molecules are weak and the compressibility $C_{s}=-\frac{1}{A}\left(\frac{\partial A}{\partial \pi}\right)_{T}$ is high. The gaseous-expanded phase is characterized by a gradual increase in surface pressure. A transition to a liquid-condensed phase follows from $\pi=6-35 \mathrm{mN} / \mathrm{m}$, and is characterized by a steeper slope and a relatively constant compressibility. A transition to a solid-condensed phase is observed above $\pi=35 \mathrm{mN} / \mathrm{m}$. The compressibility in this region is much lower, since the molecules are usually close-packed into a two-dimensional array. The film finally collapses at a surface pressure of $c a .46 \mathrm{mN} / \mathrm{m}$, corresponding to overlapping of the monolayers to form disordered films.

4.4.3 Influence of Copolymer Structure on the Compression Isotherms. While the isotherms of most copolymers displayed features characteristic of stable monolayer formation in the solid-condensed state, the composition of the copolymers had a marked influence on the transition points of the isotherms, the curves being shifted to larger surface areas per molecule with increasing PEO contents. Copolymers with high PEO contents displayed more distinct transitions, making the gaseous-expanded, the liquid-condensed, and the solid-condensed phases more clearly identifiable (Figures 4.2 and 4.3). This is apparently the result of a decreasing slope in the condensed-liquid state for increasing PEO contents. 
This effect is clearly seen in comparing the isotherms obtained for samples G1-30PS-LB-6.5, G1-30PS-LB-22, and G1-30PS-LB-31 (Figure 4.2): The liquid-condensed phase and the transition from the liquid-condensed to the solid-condensed phase become better defined at higher PEO contents. The lower PEO content samples are characterized not only by very short PEO chains, but also by the absence of crystallinity. Similar trends have been reported for PS- $b$-PEO copolymers, ${ }^{13,14-16}$ as the compression isotherms became more structured when the size of the poly(ethylene oxide) segment was increased. In some cases a plateau region, normally associated with conformation changes for the PEO chains, became more prominent.

The influence of the branched PS component of arborescent PS-g-PEO copolymers on the assembly process is not very obvious based only on the compression isotherms. Branching functionality typically had a minimal effect on the isotherms, the low and high branching functionality samples displaying similar features.

The isotherm profile obtained for sample G3-5PS-HB-43 was very different from the other copolymers. Even after spreading $400 \mu \mathrm{L}$ of solution onto the subphase the surface pressure remained very low over a wide compression range before rising suddenly, but it never increased above $6 \mathrm{mN} / \mathrm{m}$ and no erratic pressure variations characteristic of film collapse were observed under these conditions (Figure 4.4). This might be as a result of weak intermolecular interactions, and weak interactions between the molecules and the subphase ${ }^{17}$ resulting in unstable monolayers. Because of this, no further investigations were done on this sample. 


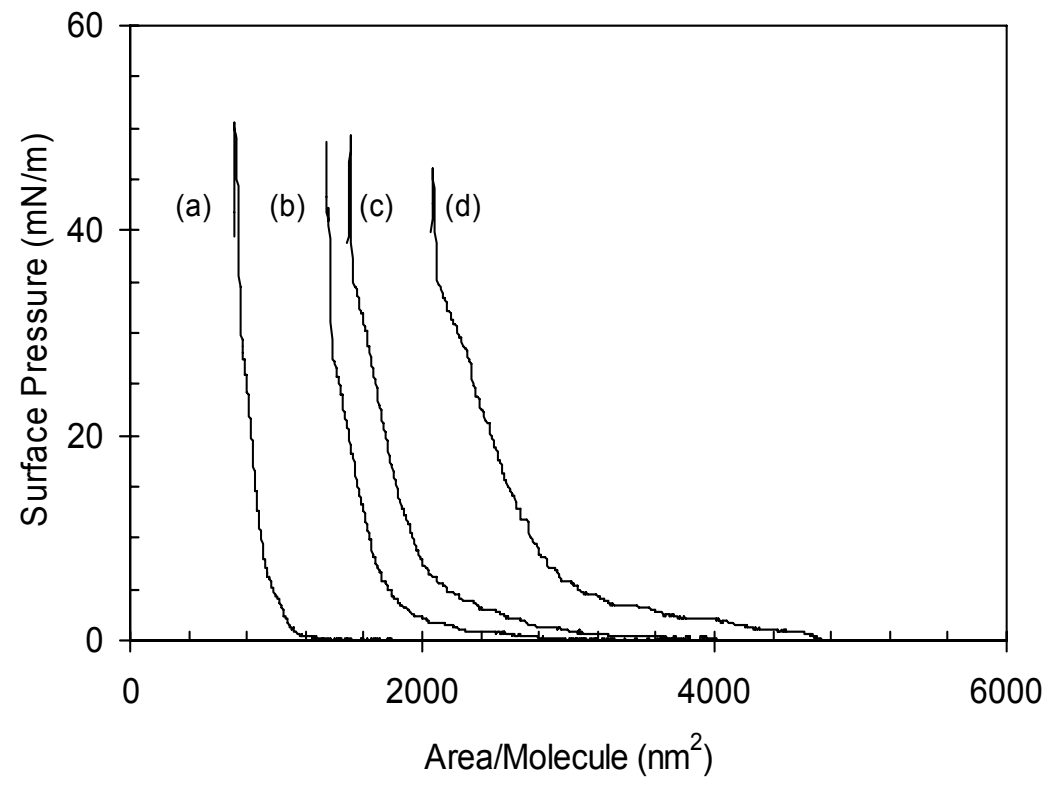

Figure 4.2. Langmuir compression isotherms at $22{ }^{\circ} \mathrm{C}$ for copolymers (a) G1-30PS-LB-6.5, (b) G1-30PS-LB-15, (c) G1-30PS-LB-22, and (d) G1-30PS-LB-31.

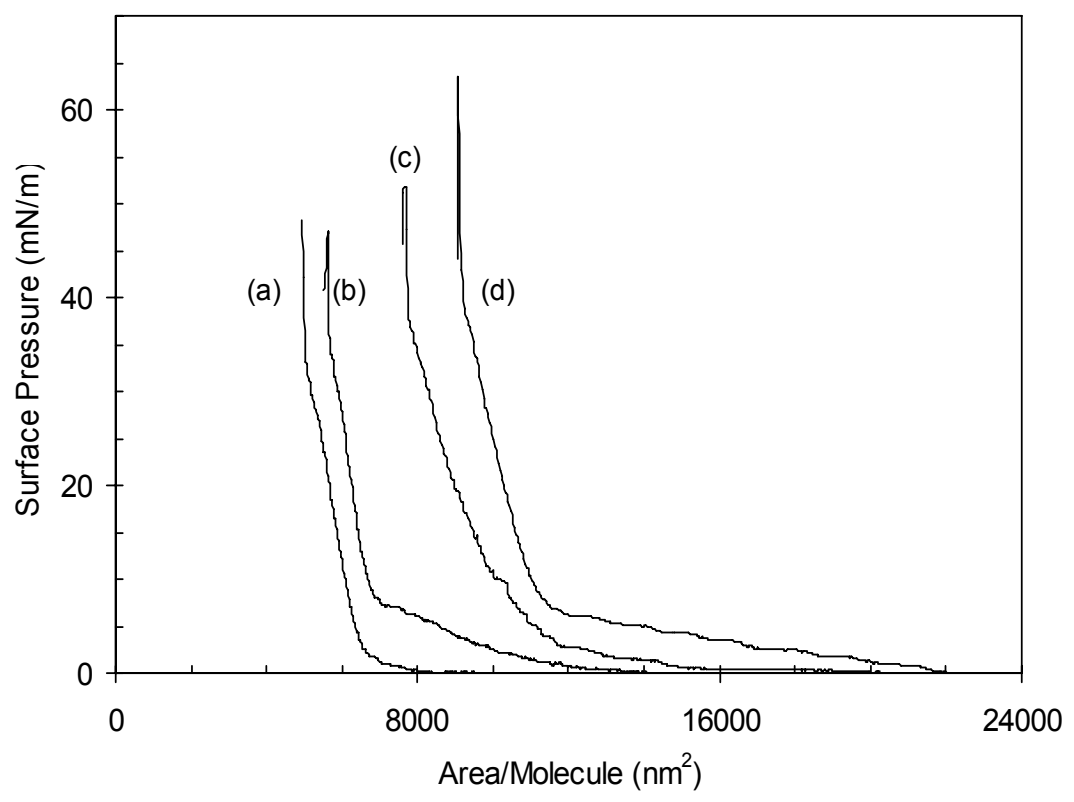

Figure 4.3. Langmuir compression isotherms at $22{ }^{\circ} \mathrm{C}$ for copolymers (a) G1-30PS-HB-8, (b) G1-30PS-HB-24, (c) G1-30PS-HB-33, and (d) G1-30PS-HB-43. 


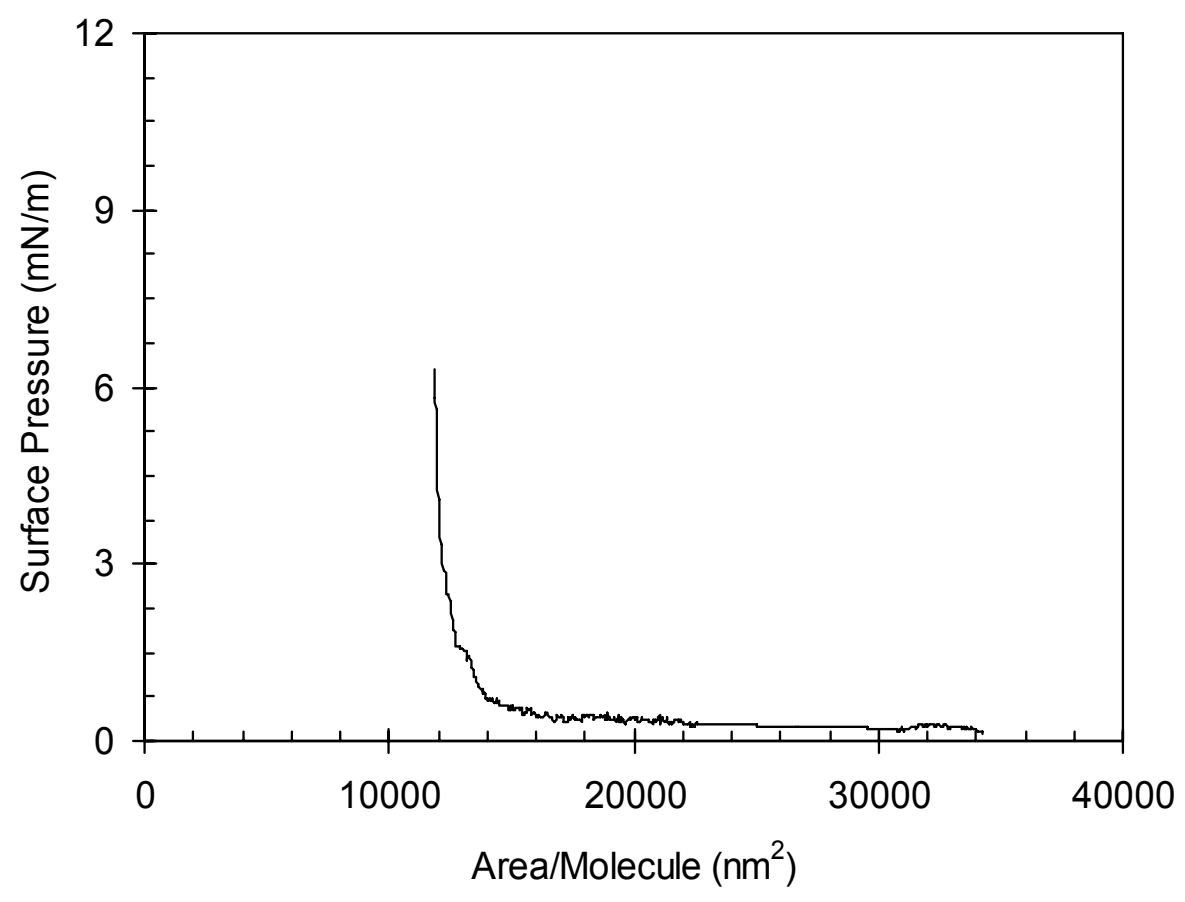

Figure 4.4. Langmuir compression isotherm at $22{ }^{\circ} \mathrm{C}$ for copolymer G3-5PS-HB- 43 with $400 \mu \mathrm{L}$ of $0.5 \mathrm{mg} / \mathrm{mL}$ solution spread on the subphase.

4.4.4 Surface Compressional Modulus. The 2-dimensional (surface) compressional modulus, corresponding to the reciprocal of surface compressibility $\left(C_{\mathrm{s}}^{-1}\right)$, is a measure of the elastic behavior of monolayer films at the air-water interface. ${ }^{18-21}$ Films with high $C_{\mathrm{s}}{ }^{-1}$ values have a lower elasticity. This parameter has also been employed to categorize the state of Langmuir monolayers, ${ }^{22,23}$ modulus $\left(C_{\mathrm{s}}{ }^{-1}\right)$ values between 12.5 and $50 \mathrm{mN} / \mathrm{m}$ being typical of the liquid-expanded state. The liquid-condensed state is characterized by $C_{\mathrm{s}}^{-1}$ values within the limits of $50-250 \mathrm{mN} / \mathrm{m}$, while $C_{\mathrm{s}}^{-1}$ values above $250 \mathrm{mN} / \mathrm{m}$ are usually obtained for monolayers in the solid-condensed state.

To study the elastic behavior of arborescent PS- $g$-PEO monolayers, $C_{\mathrm{s}}^{-1}$ was determined for the various states of the monolayers near the maximum surface pressure for 
each region. From Table 4.3 it is clear that the monolayers are all in a solid-condensed state in the terminal (high $\pi$ ) position of the isotherm, as the compression moduli measured at film collapse all exceed $250 \mathrm{mN} / \mathrm{m}$. Depending on the area per molecule, a gaseous phase with $C_{\mathrm{s}}^{-1}$ reaching $7 \mathrm{mN} / \mathrm{m}$, and a liquid-condensed state with $C_{\mathrm{s}}^{-1}$ between $80-170 \mathrm{mN} / \mathrm{m}$ were also observed. The $C_{\mathrm{s}}^{-1}$ values measured at film collapse are significantly higher for the highly branched samples than for the low branching functionality copolymers. For both the LB and the HB samples, the values tend to increase with molecular weight. This is not surprising, since the compressional modulus of materials is known to increase with both molecular weight and branching density. ${ }^{20}$ The unusually high compression modulus observed for G1-30PS-LB-22 copolymer (Table 4.3) could be linked to its high initial degree of crystallinity, since the modulus of materials typically increases with crystallinity. ${ }^{24}$ The same reasoning applies to samples G1-30PS-HB-24 and G1-30PS-HB-33.

Table 4.3. Surface Compression Moduli for the Gaseous, Liquid, and Solid States of Arborescent PS- $g$-PEO Copolymer Monolayers.

\begin{tabular}{lccc}
\hline Sample & \multicolumn{3}{c}{ Compressional Modulus $\boldsymbol{C}_{\mathbf{s}}^{-1}(\mathbf{m N} / \mathbf{m})$} \\
\cline { 2 - 4 } & Gaseous & Liquid & Solid \\
\hline G1-30PS-LB-6.5 & 2 & 115 & 384 \\
G1-30PS-LB-15 & 2 & 117 & 401 \\
G1-30PS-LB-22 & 3 & 106 & 1729 \\
G1-30PS-LB-31 & 3 & 89 & 763 \\
G1-30PS-HB-8 & 4 & 81 & 1080 \\
G1-30PS-HB-24 & 7 & 138 & 3025 \\
G1-30PS-HB-33 & 4 & 107 & 2621 \\
G1-30PS-HB-43 & 7 & 170 & 4218 \\
\hline
\end{tabular}




\subsubsection{Conformation of Arborescent PS-g-PEO Copolymers at the Air-water}

Interface. The molecular areas determined from the $\pi$ - $A$ isotherms can be used to deduce the molecular orientation and configuration of the copolymer molecules in the Langmuir monolayers. ${ }^{25-28}$ The limiting area per molecule $A_{0}$, usually calculated by extrapolation of the steep portion of the $\pi$ - $A$ curves to zero surface pressure (Figure 4.5), is used to estimate the molecular area in the solid-condensed state of the monolayer. This concept can be extended to the liquid-condensed phase to estimate the molecular area $A_{\mathrm{L}}$ in that region.

The limiting diameter $D_{0}$, and the molecular diameter $D_{\mathrm{L}}$ obtained from the liquid portion of the $\pi$-A plot (Table 4.4) were both calculated from $A_{0}$ and $A_{\mathrm{L}}$, respectively.

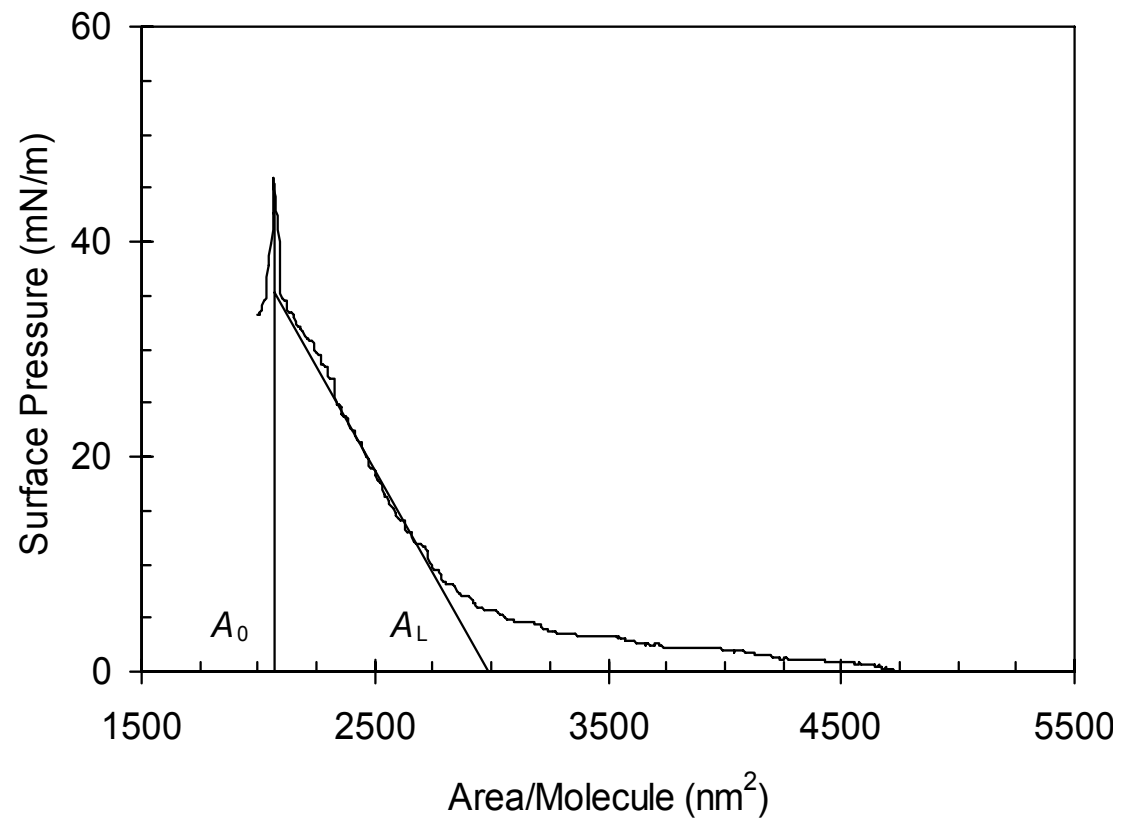

Figure 4.5. Calculation of limiting area per molecule $\left(A_{0}\right)$ and area per molecule in the liquid phase $\left(A_{\mathrm{L}}\right)$ using the compression isotherm of copolymer $G 1-30 \mathrm{PS}-\mathrm{LB}-31$ as an example. 
Apart from the presence of a solid-condensed region, the compression isotherms obtained for arborescent PS- $g$-PEO copolymers are quite similar to those of PS-PEO block and star-block copolymers with a low PEO content. ${ }^{15,29,30}$ As discussed in Chapter 3, the PEO chains of arborescent PS-g-PEO copolymers are expected to remain at the air-water interface at low surface pressures (gaseous-expanded phase) in analogy to amphiphilic linear and starblock PS-PEO copolymers. It has indeed been shown that the flexible PEO chains are partly extended and adsorbed on the water surface to form a corona surrounding the collapsed PS

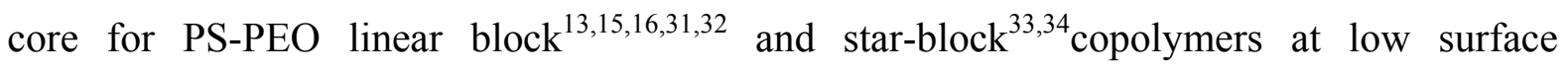
pressures, due to the strong interactions between water molecules and the PEO segments. As the surface area is decreased, the molecules are brought in closer contact and the chains should change their conformation gradually to minimize steric interactions between the PEO chains of adjacent molecules. For that reason, the surface pressure increases progressively in the gas-like region of the isotherm. The subsequent behavior of the PEO segments at the airwater interface upon further compression (liquid-condensed phase) is still contentious, especially for PS-PEO block copolymers. For example Bijsterbosch et al. ${ }^{32}$ and Gonçalves da Silva et al. ${ }^{13 a}$ suggested that the PEO chains are forced to desorb into the water subphase in this region, the chains adopting a more extended or brush-like conformation in agreement with the de Gennes scaling theory of grafted polymers at solid-liquid interfaces. ${ }^{35}$ This behavior of PEO chains at high compression has been accepted by many other groups, ${ }^{14,31,36}$ and associated with the sharp rise in surface pressure observed in the liquid-condensed phase of the isotherm. The monolayer thickness increases with the surface pressure, and $A_{0}$ is believed to represent the area occupied by the PS segments at the interface. ${ }^{34}$ In contrast, Cox 
et al. ${ }^{15,30}$ suggested that even at high compressions the PEO chains should remain adsorbed at the air-water interface.

It appears therefore likely that as compression increases from the gas-like to the liquid-condensed phase, the PEO segments of arborescent PS-g-PEO may adopt a more coiled conformation, be pushed aside of the PS cores at the air-water interface, and finally be forced to desorb at least partly into the water subphase. Considering the high branching functionality of arborescent copolymers however, it appears unlikely that complete desorption of the PEO segments into the water subphase would be possible even at high pressures.

Table 4.4. Molecular Diameters and Collapse Pressures of Langmuir Monolayers.

\begin{tabular}{lcccc}
\hline \multicolumn{1}{c}{ Sample } & $\boldsymbol{D}_{\mathbf{0}}(\mathbf{n m})^{\mathbf{a}}$ & $\boldsymbol{D}_{\mathbf{L}}(\mathbf{n m})^{\mathbf{a}}$ & $\boldsymbol{\pi}_{\mathbf{c}}(\mathbf{m N} / \mathbf{m})$ & $\boldsymbol{D}_{\text {hard sphere }}(\mathbf{n m})$ \\
\hline $\boldsymbol{G 1 - 3 0 P S - L B - 6 . 5}$ & 31 & 35 & 50 & 19 \\
$\boldsymbol{G 1 - 3 0 P S - L B - 1 5}$ & 42 & 47 & 49 & \\
$\boldsymbol{G 1 - 3 0 P S - L B - 2 2}$ & 45 & 52 & 49 & \\
$\boldsymbol{G 1 - 3 0 P S - L B - 3 1}$ & 53 & 61 & 46 & \\
$\boldsymbol{G 1 - 3 0 P S - H B - 8}$ & 81 & 91 & 48 & 30 \\
$\boldsymbol{G 1 - 3 0 P S - H B - 2 4}$ & 86 & 96 & 47 & \\
$\boldsymbol{G 1 - 3 0 P S - H B - 3 3}$ & 101 & 114 & 51 & \\
$\boldsymbol{G 1 - 3 0 P S - H B - 4 3}$ & 108 & 123 & 63 & \\
\hline
\end{tabular}

${ }^{\mathrm{a}} D_{0}$ and $D_{\mathrm{L}}$ values calculated from $A_{0}$ and $A_{\mathrm{L}}$, respectively, as shown on Figure 4.5 .

The $D_{0}$ and $D_{\mathrm{L}}$ values listed in Table 4.4 show clearly that the limiting areas increase with the PEO content for both the low and high branching functionality samples incorporating identical PS cores. The $D_{0}$ values for the LB samples are lower than the hydrodynamic diameters $\left(D_{\mathrm{h}}\right)$ reported for the cores in Table 4.1, obtained from dynamic 
light scattering measurements in solution. This is presumably due to collapse of the polystyrene cores at the air-water interface. In spite of their molecular rigidity, considerable expansion has been observed for G1-30PS arborescent polymers in toluene. ${ }^{9}$ It was likewise suggested that the PS block of PS- $b$-PEO copolymers would collapse into spherical domains at the air-water interface. ${ }^{34}$ Additionally, the low branching functionality samples have lower $C_{\mathrm{s}}^{-1}$ values reflecting a greater compressibility, possibly due to mixing of the PS and the PEO phases, and a much more diffuse PS-PEO interface, and hence should be more deformable upon compression. Another important parameter influencing the dimensions of the molecules at the air-water interface is molecular flattening (as observed by AFM imaging in Chapter 3), which should be more important for the more flexible LB samples. The hypothetical hard core diameters reported in Table 4.4 were calculated for collapsed polystyrene molecules with bulk density $\rho=1.05 \mathrm{~g} / \mathrm{cm}^{3}$ and the molecular weights listed in Table 4.1. The experimental limiting molecular diameters $D_{0}$ are intermediate between $D_{\mathrm{h}}$ and the theoretical hard core diameters, and therefore consistent with a collapsed, flattened structure on the water surface as observed in Chapter 3.

Since the diameters $D_{0}$ and $D_{\mathrm{L}}$ increase for both the LB and the HB samples with increasing PEO content in the copolymers, it could be tempting to conclude that the PEO chains remain in a coiled conformation at the air-water interface even at high compressions. In this conformation, the average height of the molecules (overall thickness of the film) should remain constant throughout the isotherm. The thickness of Langmuir-Blodgett monolayers was determined from bearing analysis ${ }^{37}$ of AFM pictures for sample G1-30PSHB-43 after transfer to mica substrates before monolayer compression, and at surface pressures corresponding to the gas-like and, liquid-condensed states. Bearing analysis is 
useful because it provides information on the statistical distribution of surface heights over a sample, unlike cross-section analysis which provides information for a surface profile along a straight line and may vary with sample orientation. It is generally accepted that the morphology of amphiphilic superstructures at the air-water interface remains unaffected when the films are transferred onto solid substrates. ${ }^{38}$ The overall thickness of the LangmuirBlodgett films should therefore reflect that of the Langmuir monolayers. The histograms derived from Bearing analysis (Figure 4.6) clearly show that the thickness of the monolayer increased at higher surface pressures. At $\pi=4 \mathrm{mN} / \mathrm{m}$ (gaseous-expanded phase) a large fraction of the PEO chains was still likely adsorbed on the water surface, since the film thickness was almost the same as before compression. However at $\pi=20 \mathrm{mN} / \mathrm{m}$ the thickness increased sharply, presumably due to desorption of the PEO chains into water. This result is in agreement with the work of Peleshanko et al., ${ }^{34}$ who reported that the overall thickness of monolayer films of star-branched PS-PEO copolymers increased from 0.3 to 5 $\mathrm{nm}$ as the surface pressure was increased from 0 to $25 \mathrm{mN} / \mathrm{m}$.

On the basis of the information collected for the Langmuir-Blodgett films, it appears that at low surface pressures $(\pi<7 \mathrm{mN} / \mathrm{m})$ the PEO chains of arborescent PS- $g$-PEO copolymers remain mainly adsorbed at the air-water interface, in analogy to block linear and star-branched copolymers. At higher surface pressures (condensed state), however, the PEO chains seem to partly desorb into the subphase by adopting a more extended chain conformation. Nonetheless, the fact that the limiting area per molecule $D_{0}$ does increase with the PEO content of the molecules for both the G1-30LB and G1-30HB sample series suggests that even at high compressions, a portion of the PEO chains remains adsorbed at the air-water interface. A similar suggestion was made for dendritic hybrid copolymers 
consisting of a flexible PEO chain attached at the focal point of $G 2$ and $G 3$ stearateterminated PAMAM dendrons. ${ }^{39}$ In that case, the experimental area per molecule in the condensed monolayers was larger than the theoretical dimensions calculated with the assumption that the stearate end groups were extended in the air perpendicularly to the

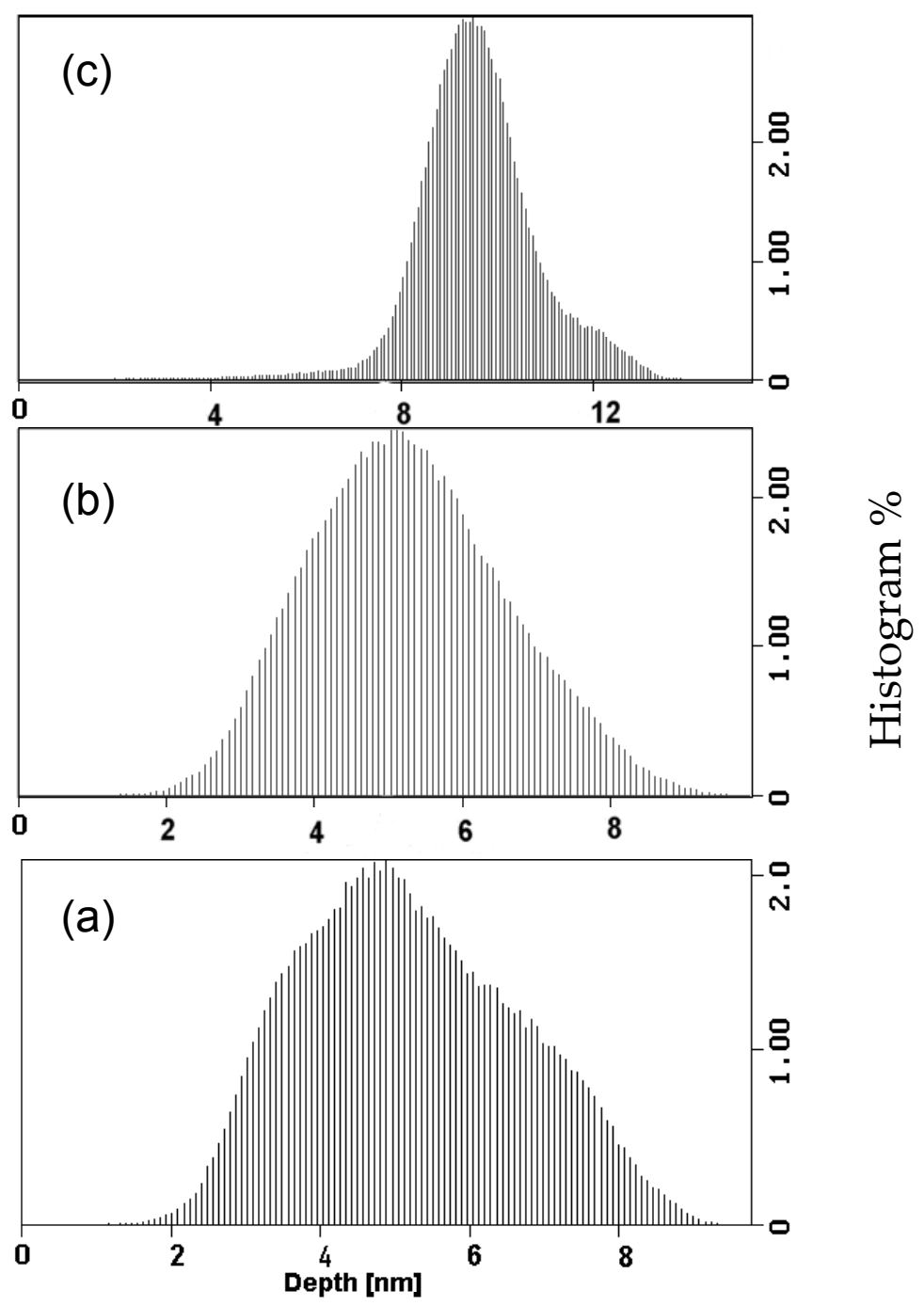

Figure 4.6. Bearing analysis on a $1.5 \times 1.5 \mu \mathrm{m}^{2}$ box area showing the monolayer thickness for G1-30HB-43 at $22^{\circ} \mathrm{C}$, (a) before compression $(\pi=0 \mathrm{mN} / \mathrm{m}$ ), (b) $\pi=4 \mathrm{mN} / \mathrm{m}$ (gaseousexpanded state), and (c) $\pi=20 \mathrm{mN} / \mathrm{m}$ (liquid-condensed state). 
interface. This led to the conclusion that some of the PEO chains were still adsorbed at the air-water interface, even though a large fraction was desorbed into water.

The influence of monolayer compression, subphase temperature, and UV irradiation of the copolymer solutions on the self-assembly behavior of arborescent copolymer micelles was examined. The study was limited to the samples with long PS side chains $\left(M_{\mathrm{w}} \approx 30000\right)$, because of their interesting association behavior noticed in Chapter 3. The profiles of the compression isotherms and the topology of the monolayers deposited on mica were both influenced by subphase temperature and UV irradiation. The behavior of G1-30PS-LB and G1-30PS-HB copolymer samples with a PEO content $\geq 22 \%$ by weight was very different from that of the low PEO content samples, however. For that reason, the influence of each parameter on the self-assembly of copolymers will be discussed separately for the high PEO content copolymers.

\subsubsection{Influence of Compression on the Topology of Langmuir-Blodgett Films of}

High PEO Content Samples. The AFM micrographs shown in Figure 4.7 were obtained from Langmuir monolayers transferred on mica at surface pressures of $0-8 \mathrm{mN} / \mathrm{m}$ for a low branching functionality sample G1-30PS-LB. At these surface pressures the monolayers are all in their gas-like state, and the PEO chains are assumed to be adsorbed on the water surrounding the PS core. ${ }^{33,34}$ Even before compression, some of the molecules associate to form short ribbon structures, in addition to the low-level aggregates and isolated molecules also present. As the surface pressure is increased, the number of isolated molecules decreases while the average length of the ribbons and the packing density both increase. A similar behavior was observed for all LB samples with PEO contents $\geq 22 \%$ by weight. Enhanced ribbon formation with increasing compression was also noticed for the highly branched 
samples (Figure 4.8), however even at surface pressures of 4 and $9 \mathrm{mN} / \mathrm{m}$, higher than for the LB samples, the ribbon-like structures were significantly shorter.
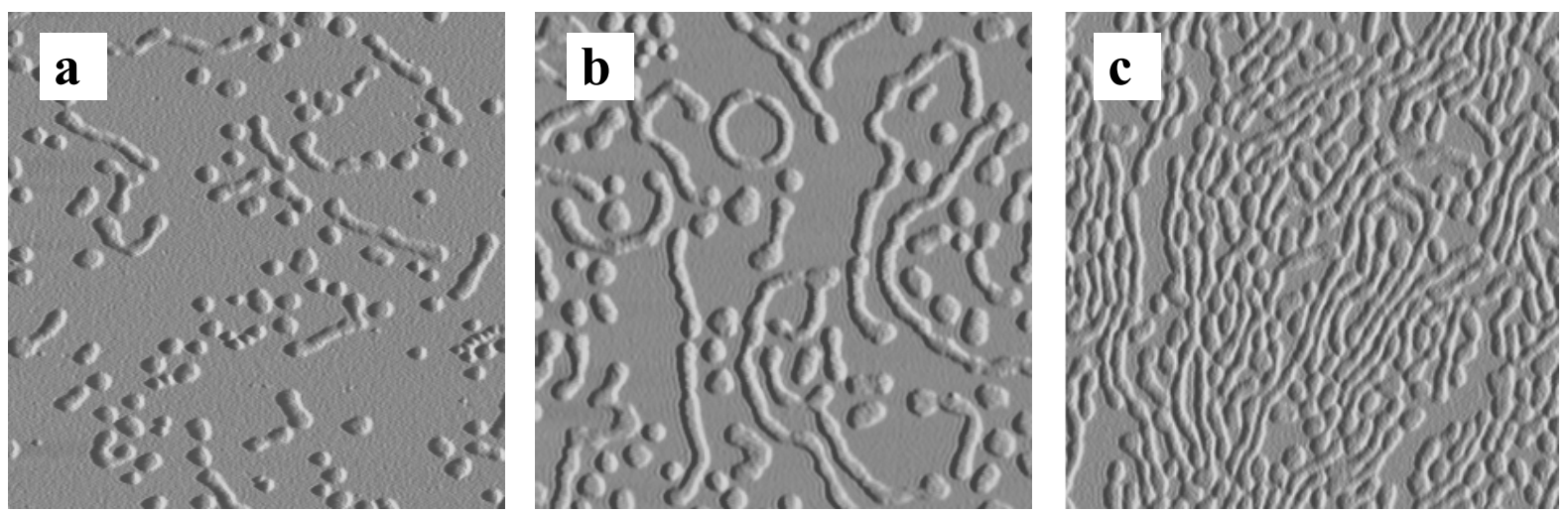

Figure 4.7. Influence of pressure on the self-assembly of high PEO content, low branching functionality copolymers: $1.5 \times 1.5 \mu \mathrm{m}^{2}$ AFM amplitude images of Langmuir-Blodgett monolayers for G1-30PS-LB-22 copolymers at (a) $0 \mathrm{mN} / \mathrm{m}$, (b) $3 \mathrm{mN} / \mathrm{m}$, and (c) $8 \mathrm{mN} / \mathrm{m}$.
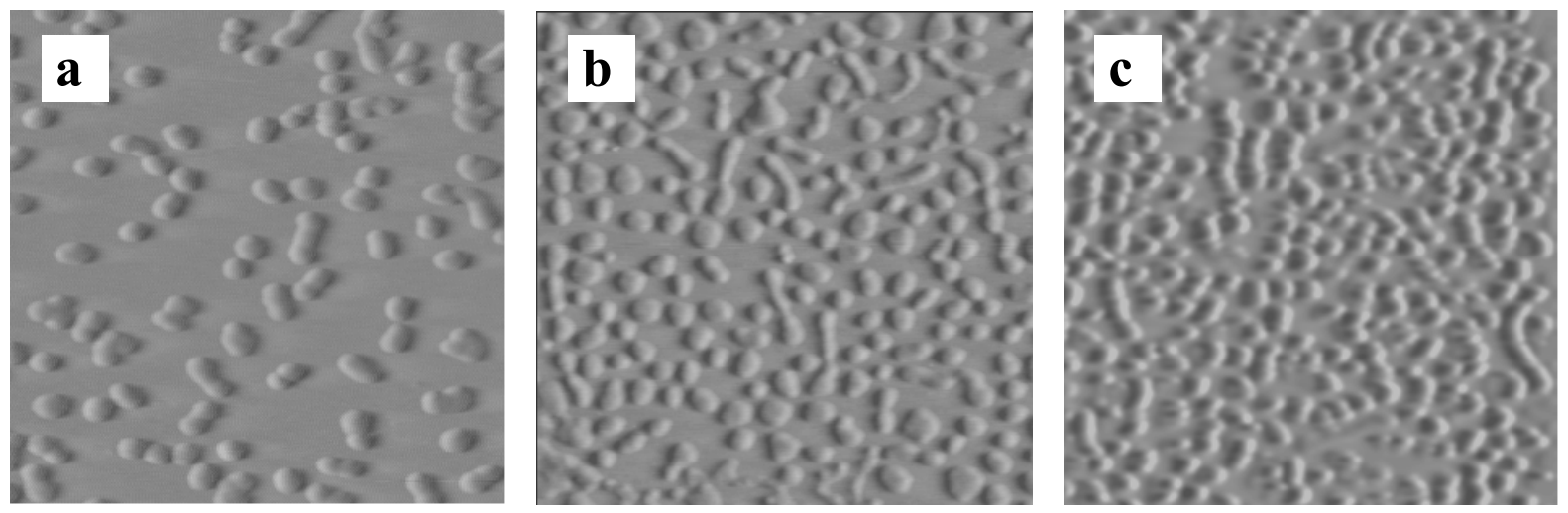

Figure 4.8. Influence of pressure on the self-assembly of high PEO content, high branching functionality copolymers: $1.5 \times 1.5 \mu \mathrm{m}^{2}$ AFM amplitude images of Langmuir-Blodgett monolayers for G1-30PS-HB-24 copolymers at (a) $0 \mathrm{mN} / \mathrm{m}$, (b) $4 \mathrm{mN} / \mathrm{m}$, and (c) $9 \mathrm{mN} / \mathrm{m}$. 
The height and width of the ribbons roughly correspond to the diameter and thickness of the individual molecules for both the LB and HB samples, indicating that ribbon formation is taking place mainly through side-by-side assembly of the molecules.

Enhanced ribbon-like structure formation with increasing surface pressure in the gaslike phase was also reported by Francis et al. for three-arm star copolymers with inner PEO segments and outer PS segments. ${ }^{40}$ Molecules of this low branching functionality copolymer spontaneously formed spherical domains at the air water-interface, but these changed into rod-like structures upon increasing the surface pressure from 0.2 to $5 \mathrm{mN} / \mathrm{m}$.

The reversibility of compression-induced ribbon formation in the gas-like phase was investigated for both the low and high branching functionality samples. Films transferred to mica were compared before compression and following a cycle of compression to $\pi=8$ and $\pi$ $=9 \mathrm{mN} / \mathrm{m}$ for the LB and HB respectively, and decompression to $\pi=0 \mathrm{mN} / \mathrm{m}$. AFM images obtained for both the LB and the HB samples looked essentially identical, without significant
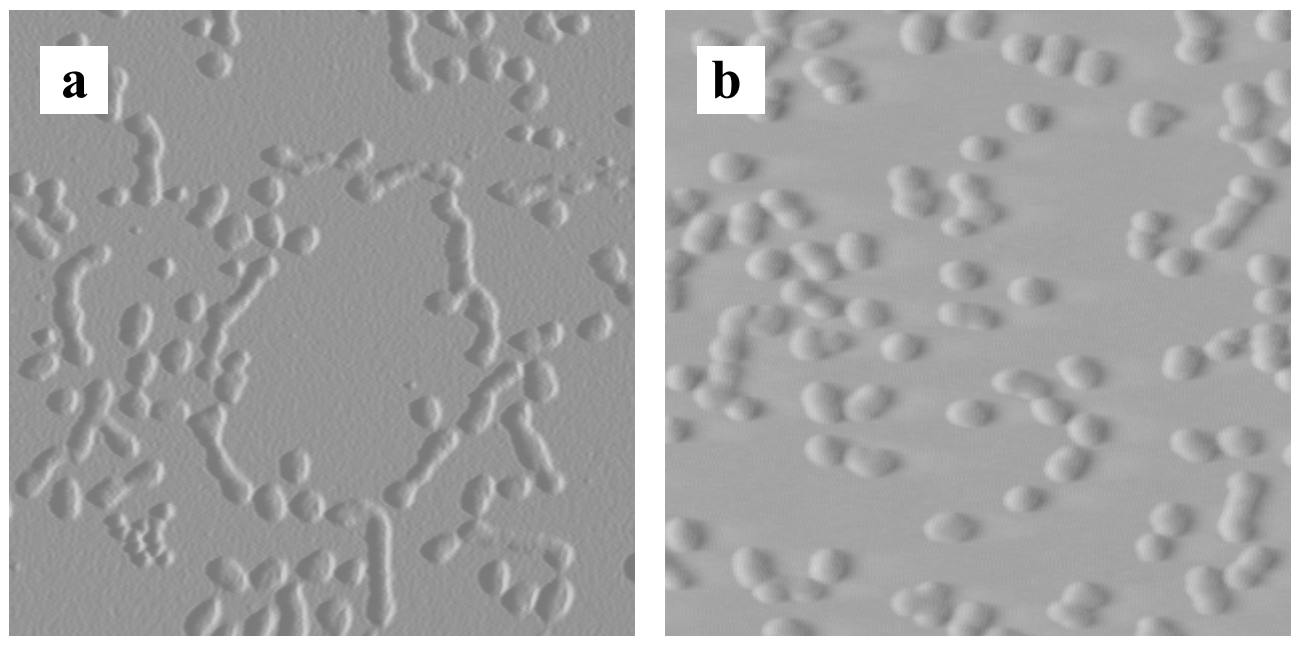

Figure 4.9. AFM pictures for (a) G1-30PS-LB-22 and (b) G1-30PS-HB-24 after decompression to $0 \mathrm{mN} / \mathrm{m}$. 
increase in the extent of association, as can be seen by comparing Figure 4.9 with Figures 4.7a and 4.8a. This clearly demonstrates that the enhanced formation of the ribbon-like features at high pressures is reversible.

A model is suggested in Figure 4.10 to account for enhanced ribbon formation at high surface pressures in the gas-like region of the Langmuir compression isotherm. The micelles on the water surface consist of a polystyrene-rich rigid core surrounded by the flexible adsorbed PEO chains. During compression, increased van der Waals attractive forces between the PS cores are counterbalanced by the elastic deformation forces of the flexible PEO chains. Ribbon growth under the influence of pressure implies that the van der Waals forces become slightly larger than the repulsive forces associated with the elastic deformation of the PEO chains under these conditions. Sideways displacement of the PEO chains leading to an increased lateral PEO chain density would ensure the exclusive formation of ribbons with a width and thickness comparable to the size of the individual molecules.

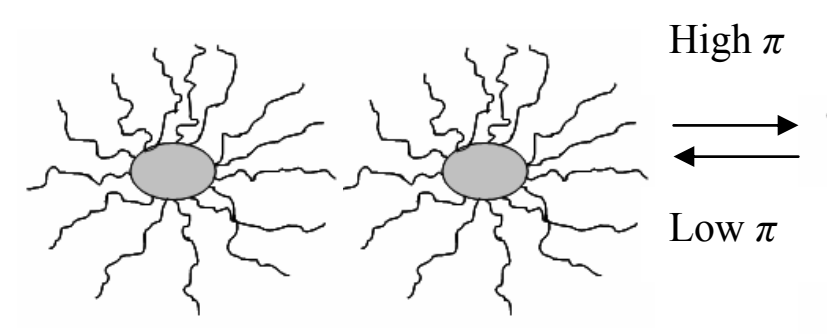

Low surface pressure

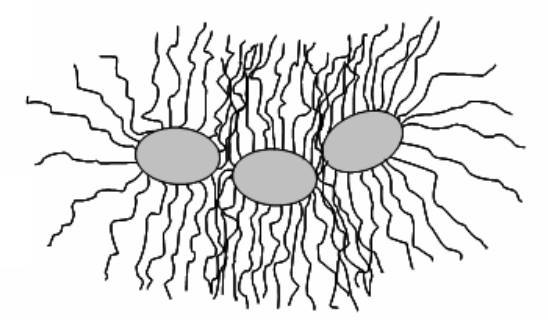

High surface pressure

Figure 4.10. Top view of the reversible association model proposed for the reversible formation of ribbon-like superstructures by HB, high PEO content samples. 


\subsubsection{Temperature Dependence of Langmuir-Blodgett Film Topology for}

Samples with High PEO Contents. The influence of temperature on film topology is illustrated in Figure 4.11 using sample G1-30PS-HB-43 as an example, after compression to a surface pressure of $4 \mathrm{mN} / \mathrm{m}$ to ensure a uniform distribution of molecules on the water. Irregularly packed molecules with minimal aggregation are observed at $12{ }^{\circ} \mathrm{C}$, while a significant number of short ribbon-like features can be identified at $37{ }^{\circ} \mathrm{C}$. All high PEO content copolymer samples displayed similar enhanced ribbon formation at higher temperatures.
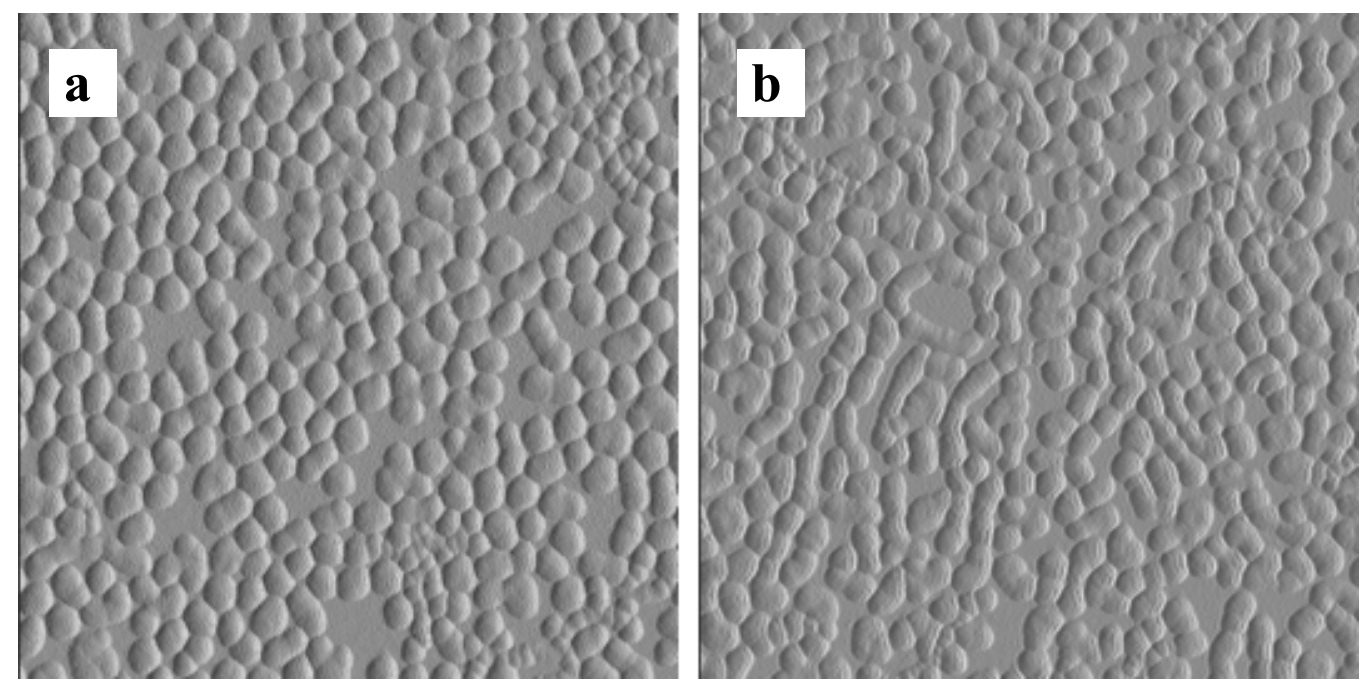

Figure 4.11. Film topology for copolymer $G 1-30 \mathrm{PS}-\mathrm{HB}-43$ at (a) $12{ }^{\circ} \mathrm{C}$ and (b) $37{ }^{\circ} \mathrm{C}$. The size of the images is $1.5 \times 1.5 \mu \mathrm{m}^{2}$.

The topology changes observed for the films of arborescent PS- $g$-PEO copolymers with a high PEO content as a function of temperature is presumably due to rearrangement of the PEO chains at the air-water interface leading to variations in their steric stabilizing 
ability. Compression isotherms for G1-30PS-HB-43 at subphase temperatures ranging from $10.5-37{ }^{\circ} \mathrm{C}$ are compared in Figure 4.12. At higher temperatures the collapse surface pressure was not reached under the experimental conditions used, and the liquid region of the isotherm shifted towards smaller molecular areas. The diameter $D_{\mathrm{L}}$ obtained by extrapolation from the liquid-condensed phase of the isotherm for G1-30PS-HB-43 decreased slightly from $D_{\mathrm{L}}=123 \mathrm{~nm}$ to $116 \mathrm{~nm}$ as the temperature was increased from 17 to $22{ }^{\circ} \mathrm{C}$. The diameters remained relatively constant $\left(D_{\mathrm{L}}=123 \pm 3 \mathrm{~nm}\right.$ and $\left.116 \pm 1 \mathrm{~nm}\right)$ within the temperature ranges of $10.5-17{ }^{\circ} \mathrm{C}$ and $22-37^{\circ} \mathrm{C}$, respectively. Similarly for copolymer G1-30PS-HB24, $D_{\mathrm{L}}$ remained approximately constant at $96 \pm 1 \mathrm{~nm}$ as the temperature was increased from 10 to $27^{\circ} \mathrm{C}$, but decreased to $92 \pm 1 \mathrm{~nm}$ within the temperature range $32-37^{\circ} \mathrm{C}$. For another copolymer sample (G1-30PS-LB-22) with a low branching functionality polystyrene core and $22 \%$ PEO content by weight, a critical temperature within the range $37-43{ }^{\circ} \mathrm{C}$ was observed, with $D_{\mathrm{L}}$ decreasing from $52 \pm 1 \mathrm{~nm}$ to $48 \pm 1 \mathrm{~nm}$.

A decrease in molecular area with increasing subphase temperature has been reported for different amphiphilic systems. ${ }^{13 a, 28,41}$ This phenomenon has been attributed to improved molecular packing due to increased molecular mobility at higher temperatures in the case of small molecule surfactants, ${ }^{28,41}$ but for PS- $b$-PEO block copolymers these changes were rather linked to conformation changes of the flexible PEO chains. ${ }^{13 a}$ In aqueous solutions the PEO chains are hydrated by water molecules, due to hydrogen-bonding interactions between the water molecules and the ether linkages of PEO. These interactions are disrupted at elevated temperatures, leading to decreased excluded volume repulsions between the PEO segments and a more compact conformation. ${ }^{42}$ The behavior of the PEO chains at the airwater interface is presumably similar to that in aqueous solution, the PEO segments adopting 
a more compact conformation leading to a decrease in $D_{\mathrm{L}}$ at elevated temperatures. In contrast to the PS- $b$-PEO block copolymers however, for which the decrease in molecular area with increasing subphase temperature was gradual, the arborescent PS-g-PEO copolymers apparently display a critical temperature above which the molecular area suddenly decreases. The critical temperature depends on the structure of the arborescent copolymers, as it varies with the PEO content and the branching functionality of the molecules. The structure-dependent critical behavior of arborescent polymers is analogous to that reported for colloidal PS particles stabilized with a steric layer of short $(M \approx 2000)$ PEO chains ${ }^{43}$ These aqueous dispersions with a PEO content of $20 \%$ by weight had an essentially constant average hydrodynamic diameter $(\approx 250 \mathrm{~nm})$ from 25 to $40^{\circ} \mathrm{C}$, since the PEO chains

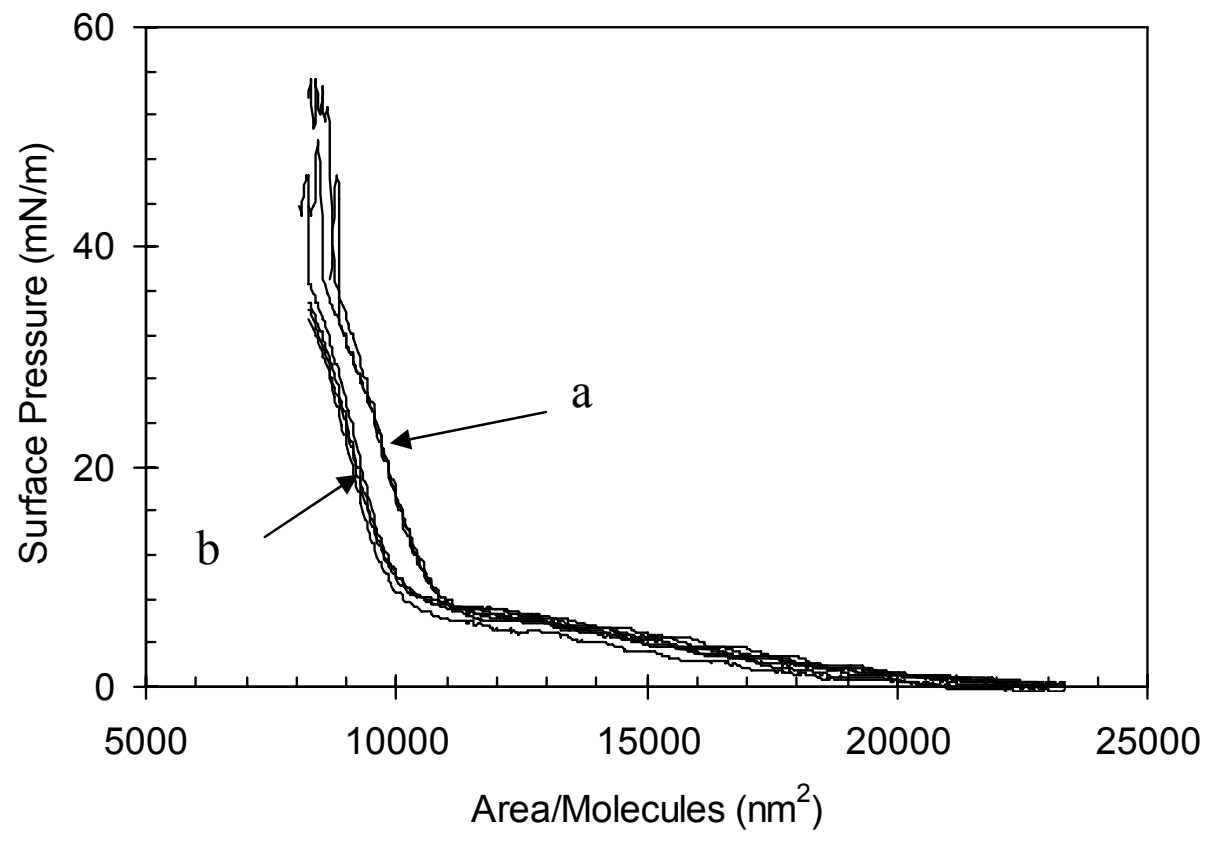

Figure 4.12. Effect of temperature on compression isotherms for copolymer G1-30PS-HB43: (a) $10.5,12$, and $17^{\circ} \mathrm{C}$; (b) $22,27,32$, and $37^{\circ} \mathrm{C}$. 
were able to stabilize the particles. This was followed by an abrupt increase to ca. $360 \mathrm{~nm}$ above $50{ }^{\circ} \mathrm{C}$ due to flocculation, once the critical temperature was reached at which the PEO segments presumably adopted a more compact conformation. The analogous behavior of arborescent PS-g-PEO copolymers buttresses the fact that these molecules behave like sterically stabilized colloids.

\subsubsection{Topology of Langmuir-Blodgett Films Obtained Upon UV Irradiation of}

the Sample Solutions. The influence of solution aging on Langmuir-Blodgett film topology for samples with high PEO contents ( $\geq 22 \%$ ) was dramatic, and is depicted in Figure 4.13 for sample G1-30PS-HB-43. Aging was initially accomplished by leaving the sample solution on the bench under ambient laboratory conditions. At predetermined time intervals, aliquots of the solution were spread on the water subphase and the monolayer was compressed to $\pi=4$ $\mathrm{mN} / \mathrm{m}$ before deposition. The subphase temperature was maintained at $22{ }^{\circ} \mathrm{C}$ in all cases.

It is clear that aging of the copolymer solution greatly enhances the formation of ribbon-like structures. The films produced after 100 days aging include exclusively ribbonlike features, some as long as $5 \mu \mathrm{m}$. The width and height of the ribbons correspond to the dimensions of single molecules as before. These structures were also observed for LangmuirBlodgett films transferred at zero surface pressure. After 130 days aging, a network-like structure (Figure 4.13f) attributed to dewetting of the copolymers from the water surface was obtained. The hydrodynamic diameter of the molecules determined from DLS measurements in chloroform was $92.7 \pm 0.5 \mathrm{~nm}$ in the sample aged for 44 days. This value was essentially identical to that obtained for a freshly prepared sample $(95.5 \pm 0.2 \mathrm{~nm})$ and confirms the lack of aggregation in solution. This implies that the self-assembly of arborescent PS- $g$-PEO copolymers into ribbon superstructures takes place only when the molecules are spread on 
the subphase. Aging of the solutions in the dark had very little or no effect on degree of association of the copolymer micelles, as the film topology obtained after 44 days aging in the dark was comparable to that observed for solutions aged 7 days under ambient laboratory lighting conditions (Figure 4.13a). This confirms that light plays a role in the formation of the long ribbon-like structures.

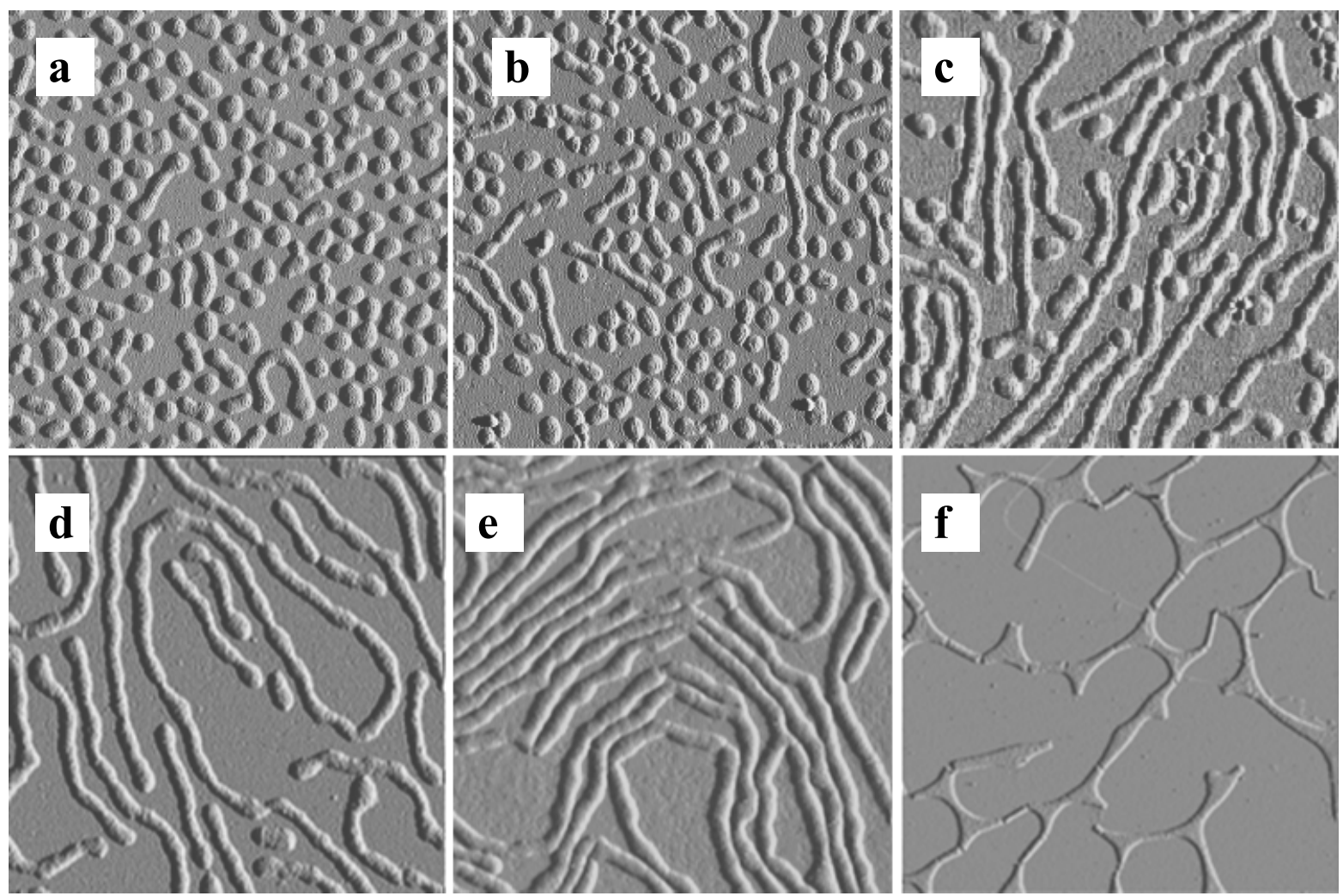

Figure 4.13. Changes in film topology for copolymer G1-30PS-HB-43 upon aging of solution under ambient conditions $\left(1.5 \times 1.5 \mu \mathrm{m}^{2}\right.$ images): (a) 7 days, (b) 20 days, (c) 34 days, (d) 44 days, (e) 100 days, and (f) $10 \times 10 \mu \mathrm{m}^{2}$ image after 130 days.

While pure poly(ethylene oxide) does not absorb near UV radiation, it becomes susceptible to photodegradation in the presence of substances able to absorb UV light. ${ }^{44}$ In 
the presence of the PS cores, the photodegradation of the PEO chains thus becomes more likely since the aromatic polymer absorbs in the near UV region. Decreased steric repulsions due to random cleavage of the PEO chains should promote association, since the van der Waals attractive forces between adjacent cores would become more significant. To verify this hypothesis, sample solutions were subjected to UV irradiation under controlled conditions before spreading on the water subphase. A series of AFM images illustrating the dependence of film topology on the UV irradiation dose in solution is provided in Figure 4.14. For this experiment six UV lamps were used at a distance of $50 \mathrm{~cm}$ from the sample solutions.

It is clear that the films are enriched with ribbon-like domains even after only $5 \mathrm{~min}$ irradiation, and that the length of the ribbons increases at longer irradiation times. The topology of the films changes from ribbon-like features to island-like clusters with a broad size distribution after 30 min of irradiation without exposure to air other than that present in the head space of the vial. Intermittent exposure of the solution to air during irradiation reduced the time required for the onset of cluster formation to $20 \mathrm{~min}$, while the results obtained with and without stirring of the solution were similar. The influence of air on the onset of cluster-like aggregate formation is again consistent with photochemical degradation of the PEO chains, since oxygen is known to participate in the degradation reaction. ${ }^{44 c}$ While film topology is not as controlled as on Figure 4.13, the influence of UV irradiation on the association process is obvious from Figure 4.14. Reducing the UV light intensity and increasing the irradiation time greatly improved the definition of the ribbons. The AFM pictures on Figure 4.15 were obtained from sample solutions irradiated with a single UV lamp at a distance of $50 \mathrm{~cm}$ from the sample. The film topology obtained is surprisingly 
similar to that on Figure 4.13. The ribbon-like features are well-defined, their length increasing with irradiation time. The behavior of other high PEO content LB and HB copolymers upon UV irradiation was similar to sample G1-30PS-HB-43. Thus by varying either the intensity of UV radiation or the irradiation time in solution, Langmuir-Blodgett films with different topologies were produced from the same arborescent PS-g-PEO copolymer sample. The enhanced association effect observed is attributed to increased
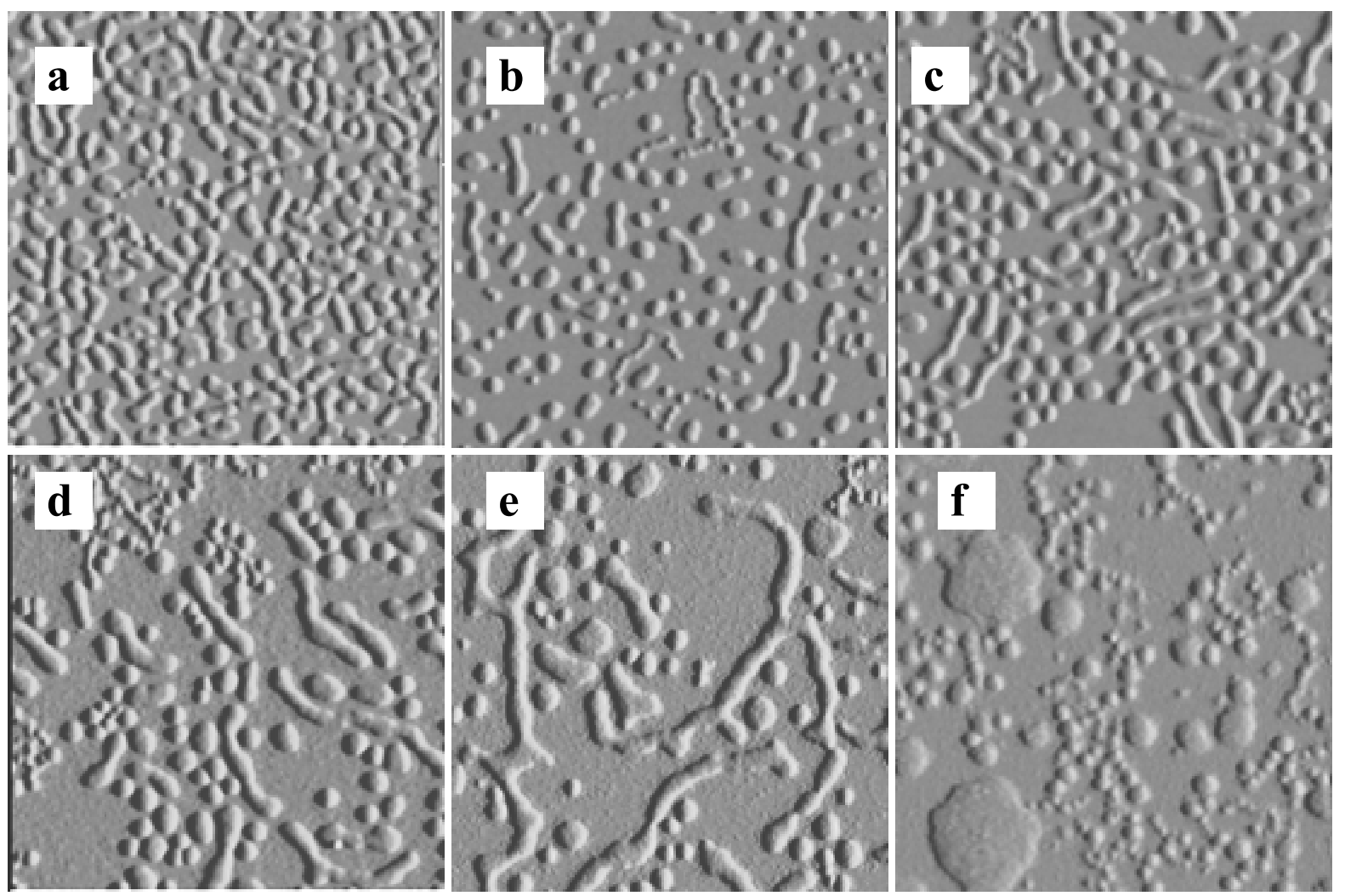

Figure 4.14. Influence of UV irradiation on Langmuir-Blodgett film topology $\left(1.5 \times 1.5 \mu \mathrm{m}^{2}\right.$ AFM pictures) for copolymer $G 1$-30PS-HB- 43 at $\pi=4 \mathrm{mN} / \mathrm{m}$ after solution irradiation at 50 cm with 6 UV lamps for (a) $0 \mathrm{~min}$, (b) $2.5 \mathrm{~min}$, (c) $5 \mathrm{~min}$, (d) $10 \mathrm{~min}$, (e) $15 \mathrm{~min}$, and (f) 30 $\min$. 
van der Waals attractions as the stabilizing PEO layer was gradually destroyed. The fate of the cleaved PEO segments is however uncertain. The cleaved PEO chain segments could either remain adsorbed on the subphase or desorb into it. While PEO is water-soluble, high molecular weight linear PEO $\left(M_{\mathrm{w}}=1.8 \times 10^{4}-5.9 \times 10^{5}\right)$ has been shown to remain adsorbed at the air-water interface at low surface pressures and to form stable monolayers up to $\pi \approx 10 \mathrm{mN} / \mathrm{m}^{45}$ An irradiated G1-30PS-HB-43 copolymer solution can be considered equivalent to a blend of sterically stabilized PS particles and free PEO linear segments.

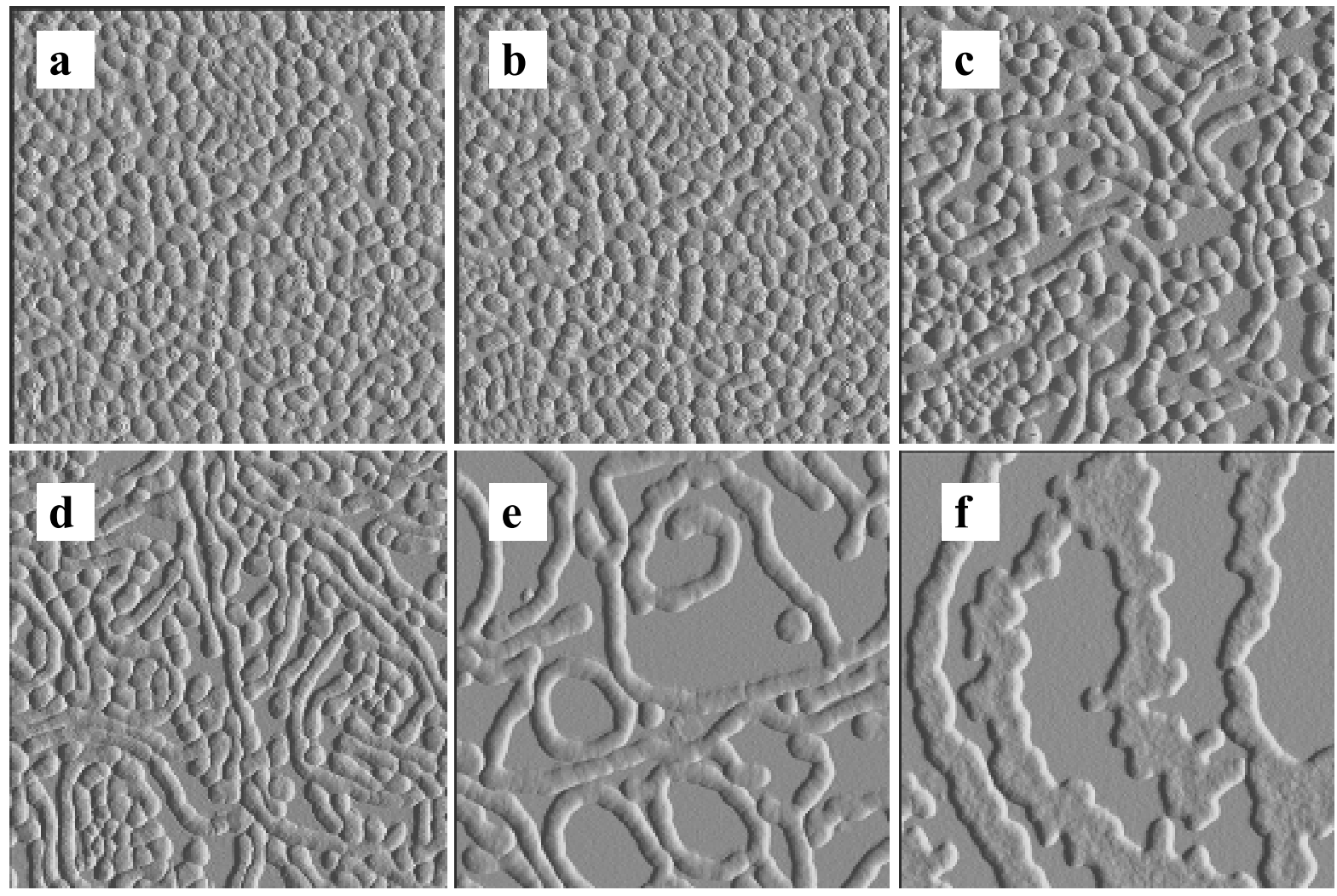

Figure 4.15. $1.5 \times 1.5 \mu \mathrm{m}^{2}$ AFM pictures for Langmuir-Blodgett films obtained for copolymer 61 -30PS-HB- 43 at $\pi=4 \mathrm{mN} / \mathrm{m}$ after solution irradiation with a single UV lamp for (a) $1 \mathrm{~h}$, (b) $2 \mathrm{~h}$, (c) $4 \mathrm{~h}$, (d) $6 \mathrm{~h}$, (e) $9 \mathrm{~h}$, and (f) $13 \mathrm{~h}$. 
The transitions in the compression isotherms were shifted to lower molecular areas as the irradiation time was increased. For G1-30PS-HB-43 for example, the molecular diameter $D_{\mathrm{L}}$ estimated from the liquid-like phase decreased from $123 \pm 4 \mathrm{~nm}$ to $114 \pm 2 \mathrm{~nm}$ after $9 \mathrm{~h}$ of irradiation with a single UV lamp at a distance of $50 \mathrm{~cm}$. This result is not only consistent with shortening of the grafted PEO chains, but also suggests that some or all the cleaved PEO segments may have desorbed into the aqueous subphase.

The addition of homopolymers has been shown to influence the self-assembly of block copolymers in solution. ${ }^{46}$ The homopolymer contaminant is usually solubilized within the micelle, leading to an increase in size or a change in topology of the assemblies obtained. For example Whitmore and Smith, ${ }^{46 a}$ in a combined theoretical and experimental study involving ternary mixtures of PS- $b$-PEO, and PEO demonstrated that the reverse micelles formed by the block copolymers in a toluene/tetrahydrofuran/methanol mixed solvent system increased in size as PEO homopolymer was solubilized in the PEO core of the micelles. Zhang and Eisenberg ${ }^{46 \mathrm{~b}}$ also reported the swelling of regular crew-cut polystyrene- $b$ poly(acrylic acid) copolymer micelles in aqueous solutions upon addition of polystyrene, as the homopolymer was solubilized within the PS cores of the micelles. However, upon addition of 5\% PS by weight to nonspherical micelles (vesicles and cylinders), the topologies changed to spheres as a result of solubilization.

In sterically stabilized colloidal systems, free polymer chains can either increase or decrease the stability of the dispersions. ${ }^{47}$ In a good solvent for the free polymer, the chains can prevent the approach of the particles and minimize van der Waals attractive forces, thus increasing colloidal stability. This phenomenon, known as 'depletion stabilization', has an effect opposite to 'depletion flocculation' whereby the particles become destabilized as the 
polymer chains are excluded from the interparticle region. ${ }^{47}$ The free polymer chains can also serve as bridging flocculants, enhancing aggregation by bridging two or more particles. ${ }^{3,47}$ To probe the potential influence of the UV-cleaved PEO chains on the association process, a series of solutions was prepared by blending arborescent PS- $g$-PEO copolymers with a linear PEO homopolymer $\left(M_{\mathrm{w}} \approx 5000\right)$. A series of AFM pictures is shown in Figure 4.16 for Langmuir-Blodgett films produced from blends of sample G1-30PS-HB-33 with increasing PEO weight fractions. Sample G1-30PS-HB-33 was selected for these experiments because of its molecular diameter $D_{\mathrm{L}}(114 \mathrm{~nm})$, identical with that determined for G1-30PS-HB-43 after irradiation with UV light for 9 hours. Before the addition of linear PEO, the films of G1-30PS-HB-33 contained mostly distinct molecules. Association was minimal as the steric PEO layer was able to provide adequate stabilization. Upon addition of a small quantity (5\% $\mathrm{w} / \mathrm{w}$ ) of linear PEO the topology changed to large irregular aggregates, and transformed into a network topology at a PEO weight fraction of $7 \%$. An increase in the lateral dimensions of the aggregates was observed for blends containing $14 \%$ and $29 \%$ PEO w/w, but the network topology was maintained. The formation of a two-dimensional network-like structure in the presence of linear PEO demonstrates that while linear PEO can reduce the colloidal stability of arborescent PS-g-PEO copolymers at the air-water interface, it is in itself unable to drive the formation of the ribbon-like superstructures. 

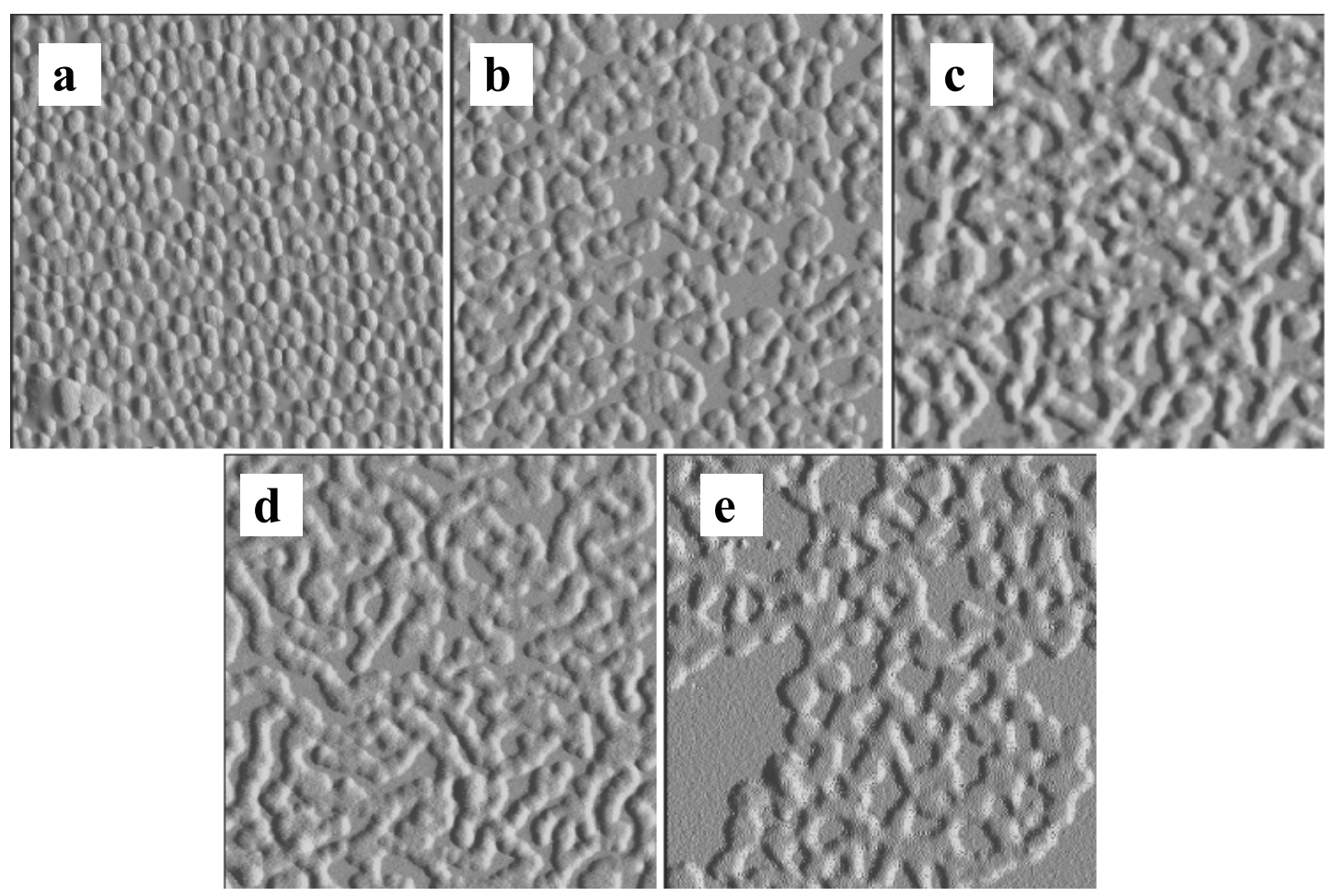

Figure 4.16. Influence of linear PEO contaminant on Langmuir-Blodgett film topology for copolymer G1-30PS-HB-43/PEO at $\pi=4 \mathrm{mN} / \mathrm{m}$ : (a) $0 \% \mathrm{w} / \mathrm{w}$ PEO, (b) $5 \% \mathrm{w} / \mathrm{w}$ PEO, (c) 7\% w/w PEO, (d) 14\% w/w PEO, and (e) $29 \%$ w/w PEO.

4.4.9 Association Behavior of Copolymers with Low PEO Contents. Samples with low PEO contents ( $\leq 15 \%$ by weight) formed large island-like clusters with a broad size distribution even before compression. As the surface pressure was increased, the clusters coalesced into larger entities as shown in Figure 4.17. This behavior is very different from that of the heteroarm (miktoarm $\mathrm{PEO}-b-\mathrm{PS}_{3}$ ) star-block copolymers (very low branching functionality copolymers) studied by Peleshanko et al. $^{34}$ at the air-water interface. At a surface pressure of $0.7 \mathrm{mN} / \mathrm{m}$, this copolymer, with a PEO content of only $11 \%$ by weight 
and long PS chains relative to the PEO segment, formed predominantly circular domains with a broad size distribution similarly to G1-30PS-LB and G1-30PS-HB samples with a PEO weight content up to $15 \%$, at zero surface pressure. However in contrast to arborescent copolymers, compression of the star-block copolymer monolayer only resulted in increased packing density without any significant changes in domain size.

An increase in domain size without compression of the arborescent samples was also observed when the copolymer solution was aged on the working bench for about 14 days, or upon direct irradiation for about 10 min with $6 \mathrm{UV}$ lamps located $50 \mathrm{~cm}$ away from the sample solution. Increasing the temperature of the subphase from $22{ }^{\circ} \mathrm{C}$ to $43{ }^{\circ} \mathrm{C}$ without compression had a similar effect, albeit to a lesser extent. When the sample solutions were aged on the bench for 35 days before spreading, the topology of the resulting LangmuirBlodgett films changed from large island-like clusters to a mesh-like structure as shown in Figure 4.18. This topology is similar to that obtained for thin arborescent polystyrene films by spin-casting on cleaved mica, and attributed to dewetting of the molecules from the polar mica substrate during solvent evaporation. ${ }^{48}$ Even though the topology of the PS-g-PEO copolymer films with low PEO contents is different from those observed for the high PEO content samples, the driving force leading to the formation of these assemblies is presumably the same. Because of the low molecular weight of the PEO chains in these samples, increased van der Waals forces as a result of compression, increased subphase temperature, or aging of the solution enhances dewetting of the molecules from the polar water surface.

Surprisingly, while variations in subphase temperature, aging of the copolymer solutions, or UV irradiation had a major influence on the film topologies observed, they had little or no effect on the shape of the compression isotherms for low PEO content samples. 

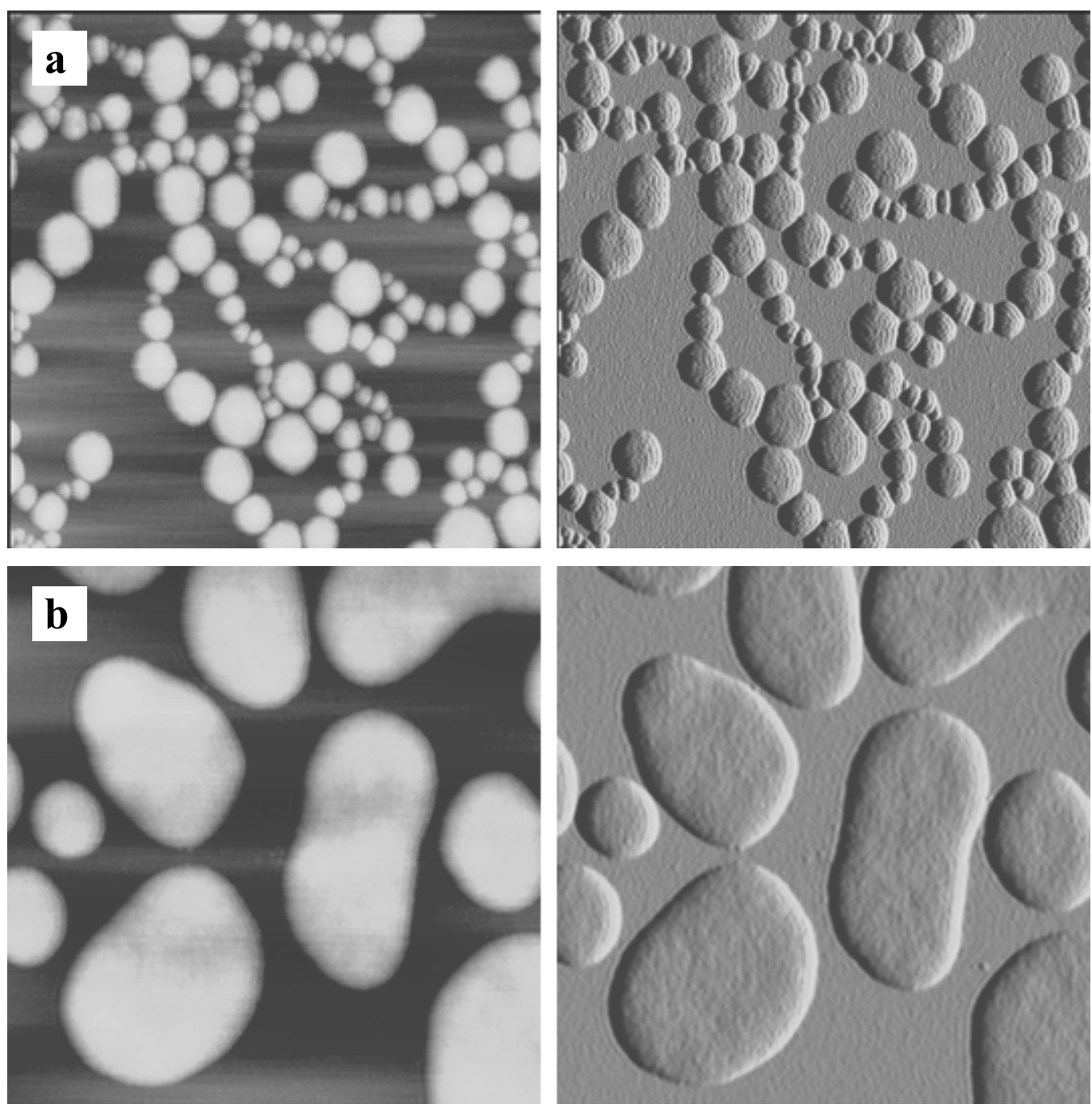

Figure 4.17. Influence of pressure on the self-assembly of low PEO content copolymers: 1.5 $\times 1.5 \mu \mathrm{m}^{2}$ height (left) and amplitude (right) images for G1-30PS-LB-15 films; (a) $\pi=0$ $\mathrm{mN} / \mathrm{m}$ and (b) $\pi=2 \mathrm{mN} / \mathrm{m}$. 

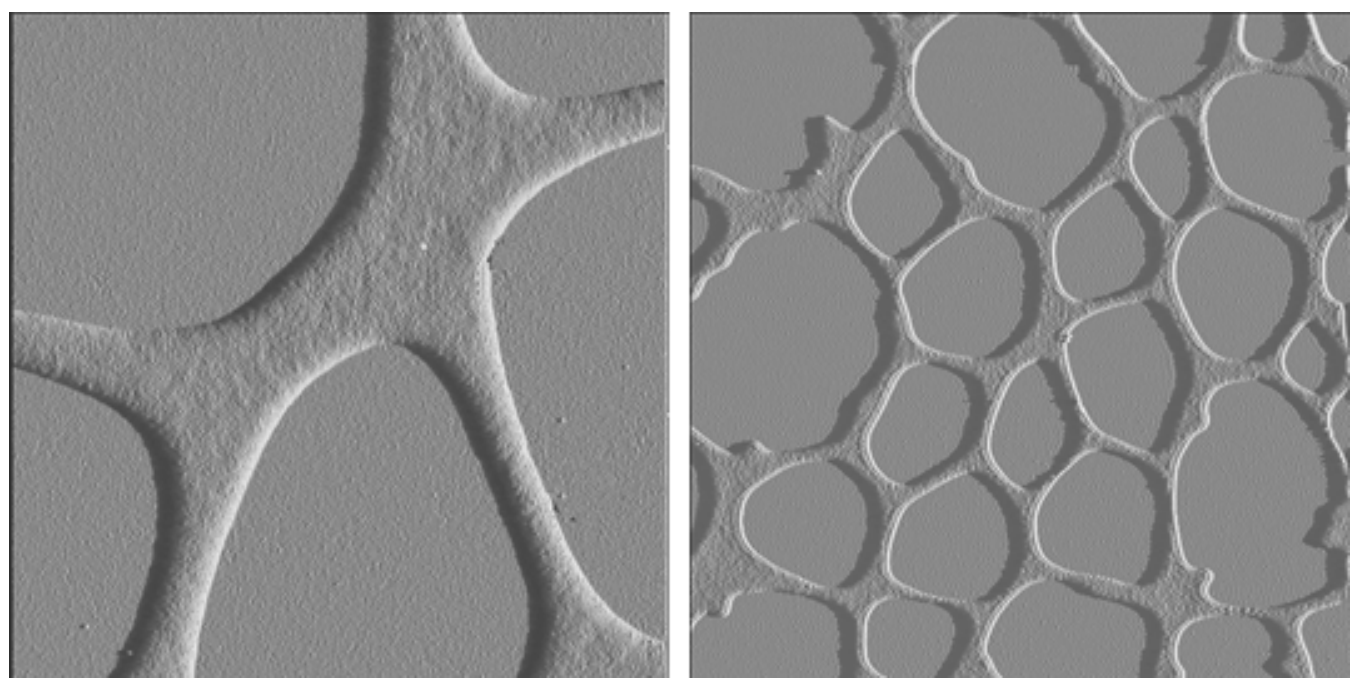

Figure 4.18. Influence of solution aging on the self-assembly of low PEO content copolymers: $2.5 \times 2.5 \mu \mathrm{m}^{2}$ (left) and $10 \times 10 \mu \mathrm{m}^{2}$ (right) amplitude micrographs of LangmuirBlodgett films for G1-30PS-HB-15 after aging of the solution for 35 days.

\subsection{Conclusions}

Arborescent polystyrene-graft-poly(ethylene oxide) copolymers self-assemble into superstructures at the air-water interface. On the basis of the variations in film thickness observed with copolymer composition, it was suggested that at low compressions the PEO chains remain mostly adsorbed at the air-water interface, but they may become partly submerged into the subphase and adopt a more brush-like conformation at very high compressions (condensed state of the monolayer), in analogy to PS- $b$-PEO block copolymers. The formation of superstructures by the dendritic micelles was enhanced by compression and, to a lesser extent, at higher temperatures. Enhanced association was most obvious when the copolymer solutions were aged by subjecting them to UV radiation. 
Depending on the composition and the structure of the copolymers, and the irradiation dose used, long ribbon-like structures or large island-like clusters were obtained at the air-water interface. The enhanced association is attributed to increased van der Waals attractive forces between the PS cores under the influence of compression, temperature, or UV irradiation. For compression and temperature-induced association, the decreased steric forces were linked to conformational changes of the PEO chains. For UV-induced assembly, however, the decreased steric repulsions were attributed to PEO chain degradation. It was ascertained from dynamic light scattering measurements that the superstructures were not formed in solution, but rather upon spreading onto the water subphase.

\subsection{Acknowledgements}

We thank the Natural Sciences and Engineering Research Council of Canada (NSERC) and the International Council for Canadian Studies (ICCS) for funding. We are also grateful to Prof. S. Mikkelsen for providing access to the atomic force microscopy equipment.

\subsection{References}

1. (a) Jones, R. A. L.; Richards, R. W. Polymers at Surfaces and Interface; Cambridge University: Cambridge, 1999, Chapters 6 and 8. (b) Koberstein, J. T. In Polymer Surfaces, Interfaces and Thin Films; Karim, A.; Kumar, S. Eds.; World Scientific: Singapore, 2000, p 115. (c) Lee. M.; Cho, B.-K.; Zin, W.-C. Chem. Rev. 2001, 101, 3869. (d) Tschierske, C. Annu. Rep. Prog. Chem. C. 2001, 97, 191. (e) Imae, T. In 
Structure-Performance Relationships in Surfactants; Esumi, K.; Ueno, M. Eds.; Marcel Dekker: New York, 2003.

2. (a) Koutsos, V.; van der Vegte, E.; Stamouli, A.; Hadziioannou, G. Macromolecules 1997, 30, 4719. (b) Seiler, M. Chem. Eng. Technol. 2002, 25, 237.

3. Israelachvili, J. Intermolecular and Surface Forces; Academic: London, 1992, Chapter 14.

4. (a) Newkome, G. R.; Moorefield, C. N.; Baker, G. R.; Johnson, A. L.; Behera, R. K.; Angew. Chem. Int. Ed. Engl. 1991, 30, 1176. (b) Teerststra, S. J.; Gauthier, M. Prog. Polym. Sci. 2004, 29, 277.

5. Sheiko, S. S.; Möller, M. Top. Curr. Chem. 2001, 212, 137.

6. (a) Gauthier, M.; Tichagwa, L.; Downey, J. S.; Gao S. Macromolecules 1996, 29, 519. (b) Cao, L. M. Sc. Thesis, University of Waterloo, Waterloo, Ontario, 1997.

7. Khasanova, A. K.; Wolf, B. A. Macromolecules 2003, 36, 6645.

8. Martuscelli, E.; Silvestre, C.; Addonizio, M. L.; Amelino, L. Makromol. Chem. 1986, $187,1557$.

9. Gauthier, M.; Li, W.; Tichagwa, L. Polymer 1997, 38, 6363.

10. Rabelero, M.; Cuenca-López, S.; Puca, M.; Mendizábal, E.; Esquena, J.; Solans, C.; López, R. G.; Puig, J. E. Polymer, 2005, 46, 6182.

11. Zhai, X.; Peleshanko, S.; Klimenko, N. S.; Genson, K. L.; Vaknin, D.; Vortman, M. Y.; Shevchenko, V. V.; Tsukruk, V. V. Macromolecules 2003, 36, 3101.

12. Hann, R. A. In Langmuir-Blodgett Films; Gareth, R. Ed.; Plenum: New York, 1990, Chapter 2. 
13. (a) Gonçalves da Silva, A. M; Filipe, J. M. d'Oliveira, A. M. R.; Martinho, J. M. G. Langmuir 1996, 12, 6547. (b) Gonçalves da Silva, A. M.; Simões Gamboa, A. L.; Martinho, J. M. G. Langmuir 1998, 14, 5327.

14. Fauré, M. C.; Bassereau, P.; Desbat, B. Eur. Phys. J. E. 2000, 2, 145.

15. Cox, J. K.; Yu, K.; Constantine, B.; Eisenberg, A.; Lennox, R. B. Langmuir 1999, 15, 7714.

16. Bassereau, P.; Fauré, M. C.; Lee, T. L.; Menelle, A.; Lheveder, C. Macromolecules 1999, 32, 8538 .

17. Kang, J. S.; Park, E. J.; Kim, J. H.; Han, M. J. Mat. Sci. Eng. C 2004, 24, 281.

18. Gaines, G. L. Insoluble Monolayers at Liquid-Gas Interfaces; Interscience: New York, 1966, p 24.

19. Behroozi, F. Langmuir 1996, 12, 2289.

20. Carino, S. R.; Duran, R. S.; Baney, R. H.; Gower, L. A.; He, L.; Sheth, P. K. J. Am. Chem. Soc. 2001, 123, 2103

21. Gómez-Serranillos, R.; Miñones Jr., J.; Dynarowicz-Łątka, P.; Iribarnegaray, E.; Casas, M. Phys. Chem. Chem. Phys. 2004, 6, 1580.

22. Davies, J. T.; Rideal, E. K. Interfacial Phenomena; Academic: New York, 1963, p 265.

23. Dynarowicz-Łątka, P.; Broniatowski, M. Polish. J. Chem. 2004, 78, 973.

24. Xu, X.; Xu. J.; Feng, L.; Chen, W. J. Appl. Polym. Sci. 2000, 77, 1709.

25. Schenning, A. P. H. J.; Elissen-Román, C.; Weener, J.-W.; Baars, M. W. P. L.; van der Gaast, S. J.; Meijer, E. W. J. Am. Chem. Soc. 1998, 120, 8199.

26. Hasegawa, T.; Hishijo, J.; Watanabe, M. Langmuir 2000, 16, 7325.

27. Sui, G.; Micic, M.; Huo, Q.; LeBlanc, R. G. Langmuir 2000, 16, 7847. 
28. Antunes, P. A.; Constantino, C. J. L.; Aroca, R. F. Langmuir 2001, 17, 2958.

29. Devereaux, C. A.; Baker, S. M. Macromolecules 2002, 35, 1921.

30. Cox, J. L.; Yu, K.; Eisenberg, A.; Lennox, R. B. Phys. Chem. Chem. Phys. 1999, 1, 4417.

31. (a) Fauré, M. C.; Bassereau, P.; Carignano, M. A.; Szleifer, I.; Gallot, Y.; Andelman, D. Eur. Phys. J. B. 1998, 3, 365. (b) Rother, G.; Findenegg, G. H. Colloid Polym. Sci. 1998, 276, 496. (c) Gragson, D. E.; Jensen, J. M.; Baker, S. M. Langmuir 1999, 15, 6127. (d) Baker, S. M.; Leach, K. A.; Devereaux, C. E.; Gragson, D. E. Macromolecules 2000, 33, 5432. (e) Akentiev, A. V.; Miller, R.; Noskov, B. A. Colloid J. 2002, 64, 653. (f) Rivillon, S.; Muñoz, M. G.; Monroy, F.; Ortega, F.; Rubio, R. G. Macromolecules 2003, 36, 4068.

32. Bijsterbosch, H. D.; de Haan, V. O.; de Graaf, A. W.; Mellema, M.; Leermakers, F. A. M.; Cohen Stuart, M. A.; van Well, A. A. Langmuir 1995, 11, 4467.

33. Francis, R.; Taton, D.; Logan, J. L.; Masse, P.; Gnanou, Y.; Duran, R. S. Macromolecules 2003, 36, 8253.

34. Peleshanko, J.; Jeong, R.; Gunawidjaja, R.; Tsukruk, V. V. Macromolecules 2004, 37, 6511.

35. de Gennes, P. G. Macromolecules 1980, 13, 1069.

36. Fauré, M. C.; Bassereau, P.; Lee, T. L.; Menelle, A.; Lheveder, C. Macromolecules 1999, 32, 8538 .

37. Digital Instrument Inc NanoScope ${ }^{\circledR}$ Command Reference Manual; Santa Barbara, 1998, p $12-46$. 
38. Petty, M. C.; Barlow, W. A. In Langmuir-Blodgett Films; Gareth, R. Ed.; Plenum: New York, 1990, Chapter 3.

39. Iyver, J.; Hammond, P, T. Langmuir 1999, 15, 1299.

40. Francis, R.; Skolnik, A. M.; Carino, S. T.; Logan, J. L.; Underhill, R. S.; Angot, S.; Taton, D.; Gnanou, Y.; Duran, R. S. Macromolecules 2002, 35, 6483.

41. Kato, T.; Tatehana, A.; Suzuki, N. Bull. Chem. Soc. Jpn. 1993, 66, 1373.

42. (a) Israelachvili, J. Proc. Natl. Acad. Sci. USA 1997, 94, 8378. (b) Branca, C.; Faraone, A.; Maisano, G.; Magazù, S.; Migliardo, P.; Triolo, A.; Triolo, R.; Villari, V. J. Phys.: Condens. Matter 1999, 11, 6079.

43. Shay, J. S.; Raghavan, S. R.; Khan, S. A. J. Rheol. 2001, 45, 913.

44. (a) Rabek, J. F. Mechanism of Photophysical Processes and Photochemical Reactions in Polymers; Wiley: London, 1987, Chapter 14. (b) Randy, B.; Rabek, J. F. J. Appl. Polym. Sci., Appl. Polym. Symp. 1979, 35, 243. (c) Kaczmarek, H. J. Photochem. Photobiol. A: Chem. 1996, 95, 61.

45. Kuzmenka, D. J.; Granick, S. Macromolecules, 1988, 21, 779.

46. (a) Whitmore, M. D.; Smith, T. W. Macromolecules 1994, 27, 4673. (b) Zhang, L.; Eisenberg, A. J. Am. Chem. Soc. 1996, 118, 3168.

47. Hirtzel, C. S.; Rajagopalan R. Colloidal Phenomena, Noyes: Park Ridge, 1985, Chapter 4.

48. Sheiko, S. S.; Gauthier, M.; Möller, M. Macromolecules 1997, 30, 2343. 


\section{Chapter 5}

\section{Arborescent Polystyrene-graft-Poly(2-vinylpyridine)}

Copolymers as Unimolecular Micelles: Solubilization

Studies 


\subsection{Abstract}

The solubilization of various polycyclic aromatic hydrocarbon (PAH) probes by arborescent polystyrene-graft-poly(2-vinylpyridine) unimolecular micelles of different structures in aqueous solutions was investigated by UV and fluorescence spectroscopy. The micelle-water partition coefficient $K_{\mathrm{w}}$ and the solubilization capacity $\kappa^{\mathrm{G}}$ both increased with the polystyrene content of the copolymers for all probes, although the increase was less significant for less hydrophobic probes. The $K_{\mathrm{w}}$ values obtained correlated well with the octanol-water partition coefficients and boiling points of the more hydrophobic probe molecules, but no clear trend was observed for $\kappa^{\mathrm{G}}$. The results obtained suggest that highly hydrophobic probes are solubilized mainly in the non-polar polystyrene core. A significant portion of the least hydrophobic probes resides in the ionic shell, while probes of intermediate polarity appear to remain in the palisade (interfacial) region. The branching functionality of the micelles played an important role in the solubilization process: For copolymers of similar composition, the solubilization capacity and rate were lower for higher generations, presumably due to the more rigid structure of the molecules. The results obtained show that arborescent polystyrene-graft-poly(2-vinylpyridine) copolymers can be designed to solubilize compounds of different polarities at controllable rates. 


\subsection{Introduction}

Polymers containing covalently bound hydrophilic and hydrophobic chain segments can self-associate into micelles when dissolved in solvents selective for one of the components. This phenomenon has sparked interest in the application of amphiphilic copolymers in different areas including the fabrication of nanoscale assemblies, the aqueous solubilization of hydrophobic compounds, drug delivery, and environmental remediation. ${ }^{1}$ Most solubilization studies have used block copolymers, and it has been established that the solubilization behavior is affected by the composition, molecular weight, and concentration of the copolymer, as well as solvent composition. ${ }^{2}$ Because solubilization by block copolymers is based on their ability to form micelles via dynamic equilibrium with unassociated species at concentrations above the critical micelle concentration (CMC), solvency conditions have a strong influence on micelle structure and stability. This limits their usefulness as drug delivery and environmental decontamination agents.

Certain amphiphilic copolymer architectures incorporating a branched component, such as the dendritic-linear block copolymer hybrids, have been shown to form multimolecular micelles analogous to block copolymer micelles by self-assembly. ${ }^{3}$ In most cases, however, dendritic polymers behave like unimolecular (non-associated) micelles maintaining their overall shape irrespective of their environment and display no CMC. Because of these remarkable characteristics, the past decade has witnessed a surge of interest in the synthesis and characterization of dendritic polymer micelles. ${ }^{4}$ These nanoscale molecules, characterized by the presence of monomeric branches (dendrimers and hyperbranched polymers) or polymeric branches (dendrigraft polymers) emanating from a 
central core, are being considered to replace block copolymers in applications such as drug delivery systems, microencapsulation, and catalysis.

One drawback of dendrimers and hyperbranched polymers is their small size (1-10 nm), making them less efficient than block copolymer micelles in certain applications. For example, micelles and liposomes with a size in the 100-200 nm range have a long blood circulation time and are able to penetrate tumor cells by different mechanisms, and are potentially more useful than dendrimers and hyperbranched polymers to target certain tissues with drugs. ${ }^{5}$

Arborescent polymers are a family of dendrigraft polymers obtained from successive grafting reactions of polymeric building blocks in a generation-based scheme analogous to dendrimers syntheses. ${ }^{6}$ These molecules have a highly branched architecture, a narrow size distribution $\left(M_{\mathrm{w}} / M_{n}<1.1\right)$, a very high molecular weight $\left(M_{\mathrm{w}}=10^{4}-10^{8}\right)$, and a hydrodynamic diameter of 10-200 $\mathrm{nm}$ attained in only a few reaction cycles. In addition, the molecules have the advantage of having many structural parameters through which their micellar properties could be varied and tailored to specific requirements. For example in a good solvent, the degree of swelling of these arborescent micelles could be varied by varying either the branching functionality, the chain length of the core and corona, or by changing the generation number. However, the effect or degree of swelling obtained by varying each of these parameters will be different. In a previous paper $^{7}$ we reported a procedure for the synthesis of arborescent polystyrenes (PS) grafted with poly(2-vinylpyridine) (P2VP) segments, using acetyl functionalities as coupling sites on the PS substrates. Dynamic light scattering measurements revealed that while copolymers derived from linear polystyrene associated into micellar superstructure when dissolved in aqueous $\mathrm{HCl}$, copolymers 
incorporating branched (generations $G 0-G 2 \mathrm{PS}$ ) substrates remained unassociated as unimolecular micelles under the same conditions. In this study, we focus our attention on the aqueous solubilization of polycyclic aromatic hydrocarbons (PAHs) (Figure 5.1) by these novel PS-g-P2VP unimolecular micelles. The influence of copolymer and probe structure on the solubilization process is investigated using UV and steady state fluorescence spectroscopy. The probes were selected in a way not only to enable the study of the effects of probe hydrophobicity, size, symmetry and physical state on micellar loading and rate of solubilization, but most importantly, to determine their solubilization sites within the micelles. Regular micelles have three distinct regions; the hydrophobic core, the hydrophilic shell, and the interfacial region between the core and the shell. Depending on the relative hydrophobicity between the probe and these regions, the solubilized probe can preferentially be located in one of these regions, and the solubilization capacity and rate of solubilization will depend on the probe location within the micelle.

\subsection{Experimental Section}

5.3.1 Sample Preparation. Anthracene (An, 99+\%), 1-bromonaphthalene (NapBr, 99\%), 1-methylnaphthalene (NapMe, 99\%), naphthalene (Nap, 99+\%), phenanthrene (Phe, 99+\%), pyrene (Py, 99\%), and 1-pyrenemethanol (PyM, 98\%) were obtained from Aldrich. Py and PyM were recrystallized twice from ethanol; $\mathrm{NapBr}$ and NapMe were distilled under reduced pressure. An, Nap, and Phe were used as received. Milli-Q water was used to prepare all samples. The arborescent PS-graft-P2VP copolymers used were synthesized by anionic grafting of $\mathrm{P} 2 \mathrm{VP}$ chains onto acetylated polystyrene substrates of different generations. ${ }^{7}$ All samples had a narrow molecular weight distribution $\left(M_{\mathrm{w}} / M_{\mathrm{n}}<1.1\right)$ and a 
polystyrene weight fraction of at most $20 \%$. Micellar solutions $(1.5 \mathrm{mg} / \mathrm{mL})$ were prepared by dissolving $75 \mathrm{mg}$ arborescent copolymer in $50 \mathrm{~mL} 0.05 \mathrm{M} \mathrm{HCl}$. After complete dissolution, $75 \mathrm{mg}$ of a PAH was added to each solution. The samples were left to equilibrate in the dark without stirring. Micellar copolymer solutions $(1.5 \mathrm{mg} / \mathrm{mL})$ without PAH were also prepared to serve as blank solutions for the UV measurements.<smiles>c1ccc2ccccc2c1</smiles>

Naphthalene (Nap)<smiles>Cc1cccc2ccccc12</smiles><smiles>c1ccc2ccccc2c1</smiles>

Phenanthrene (Phe)<smiles>c1ccc2cc3ccccc3cc2c1</smiles>

Anthracene (An)

1-Methylnaphthalene (NapMe)<smiles>Brc1cccc2ccccc12</smiles>

1-Bromonaphthalene (NapBr)<smiles>c1cc2ccc3cccc4ccc(c1)c2c34</smiles>

Pyrene (Py)<smiles>OCc1cc2ccc3cccc4ccc(c1)c2c34</smiles>

1-Pyrenemethanol (PyM)

Figure 5.1. Structures of polyaromatic hydrocarbons used.

5.3.2 Fluorescence and UV Measurements. Aliquots $(3 \mathrm{~mL})$ of the micellar solutions were drawn at predetermined time intervals and filtered using a $0.22 \mu \mathrm{m}$ pore size mixed cellulose ester (Millipore AAWP) membrane filter. Hellma quartz cells $(1.0 \times 1.0$ $\mathrm{cm}^{2}$ ) were used for the fluorescence and UV measurements. The UV measurements were carried out on a Hewlett-Packard 8452A Diode Array Spectrophotometer after dilution of the 
samples to obtain absorbance readings below 1.5. Normalized absorbance values were calculated to report the results by multiplying the actual absorbance reading by the dilution factor necessary to bring the absorbance below 1.5. Hydrophobic probe uptake was monitored until the readings became constant. Steady-state fluorescence studies were carried out using a Photon Technology International LS-100 spectrometer with a pulsed xenon flash lamp light source. The samples were deoxygenated for $20 \mathrm{~min}$ by bubbling with nitrogen. Dilution was necessary in most cases to reduce the optical density of the solutions to 0.3 or less before the fluorescence measurements, to minimize inner filter effects. For both Py and PyM fluorescence emission spectra the excitation wavelength was set at $344 \mathrm{~nm}$, and to avoid inconsistencies due to lamp intensity variations, the fluorescence intensity of a Py standard was measured before each sample.

The solubility of PyM and $\mathrm{Py}$ in $0.05 \mathrm{M} \mathrm{HCl}$ was estimated from a calibration curve obtained in pure water from fluorescence measurements following the protocol of Kwon et al. ${ }^{8}$ A stock solution of each compound was prepared by first dissolving $2.40 \mathrm{mg}$ PAH in HPLC grade acetone, after which the acetone was evaporated. Milli-Q water $(250 \mathrm{~mL})$ was added and the solution was stirred overnight for equilibration. The solution was then filtered through a $0.22 \mu \mathrm{m}$ mixed cellulose ester filter to remove residual crystals. The concentration of the saturated aqueous solution was confirmed by UV absorption measurements. Sample solutions of different concentrations were then prepared by dilution of aliquots of the stock solution with Milli-Q water. Steady-state measurements were carried out on the solutions and the intensity of the emission peak at $373 \mathrm{~nm}\left(I_{1}\right)$ was used to establish calibration curves for both PyM and Py. 


\subsection{Results and Discussion}

It has been shown that amphiphilic dendrimers are capable of greatly enhancing the aqueous solubility of hydrophobic molecules. ${ }^{9}$ Encapsulation by these unimolecular micelles may rely on physical entrapment of the guest molecules, the formation of non-covalent interactions between the guest molecules and the carrier, or hydrophobic interactions. ${ }^{4 \mathrm{~d}}$ For dendrimers, the absorption capacity is simply a function of the number of internal cavities available for entrapment, which depends on the generation number. The situation becomes more complex when dealing with charged micellar systems. ${ }^{10}$ For example block copolymer micelles with a polyelectrolyte shell have a larger size than non-ionic copolymer micelles, despite their smaller aggregation number. The micellar properties of such systems are strongly influenced by the nature of the corona that, in turn, is sensitive to the $\mathrm{pH}$ and ionic strength of the solution. ${ }^{11}$ Arborescent PS-g-P2VP copolymers have been found to expand upon ionization with $\mathrm{HCl}$, in analogy to polyectrolyte micelles. ${ }^{12}$ In the present study we examined the solubilization of a series of PAHs by arborescent PS-g-P2VP copolymers in acidified aqueous solutions. The influence of probe size, symmetry, and substituents on the solubilization process has been examined. Two fundamental parameters used for this purpose are the solubilization capacity $\kappa^{\mathrm{G}}\left(\mathrm{mg}\right.$ probe/g micelle) and the partition coefficient $K_{\mathrm{w}}{ }^{13}$

$$
\begin{aligned}
\kappa^{G} & =\frac{S_{t o t}^{G}-S_{s}^{G}}{C_{m i c}^{G}-C M C^{G}} \\
K_{W} & =\frac{S_{m i c}}{S_{s}}
\end{aligned}
$$

where $S_{\text {tot }}^{G}$ is the total concentration of the probe in the micellar solution $(\mathrm{mg} / \mathrm{mL}), S_{s}^{G}$ is the concentration of the probe in the solvent $(\mathrm{mg} / \mathrm{mL}), S_{\text {mic }}$ is the number of moles of probe 
solubilized per gram of micelles, and $S_{s}$ is the number of moles of probe dissolved per gram of solvent. In the present case the micelle concentration $C_{m i c}^{G}(\mathrm{~g} / \mathrm{mL})$ is set to the copolymer concentration, since the critical micelle concentration $C M C^{G}(\mathrm{~g} / \mathrm{mL})$ is zero for unimolecular micelles. The architecture of some copolymers of the type used in the study is compared in Figure 5.2. These copolymers were obtained from successive anionic grafting reactions as described previously. ${ }^{7}$ The characteristics of the copolymers used are summarized in Table 5.1. The nomenclature used for arborescent copolymers identifies their composition and structure. For example, G1PS-P2VP5-13 refers to a graft copolymer synthesized from a $G 1$ polystyrene core $\left(M_{\mathrm{w}} \approx 5000\right.$ linear PS grafted twice with $M_{\mathrm{w}} \approx 5000$ PS side chains), coupled with $M_{\mathrm{w}} \approx 5000 \mathrm{P} 2 \mathrm{VP}$ side chains in a third grafting reaction, with a PS content of $13 \%$ by weight.

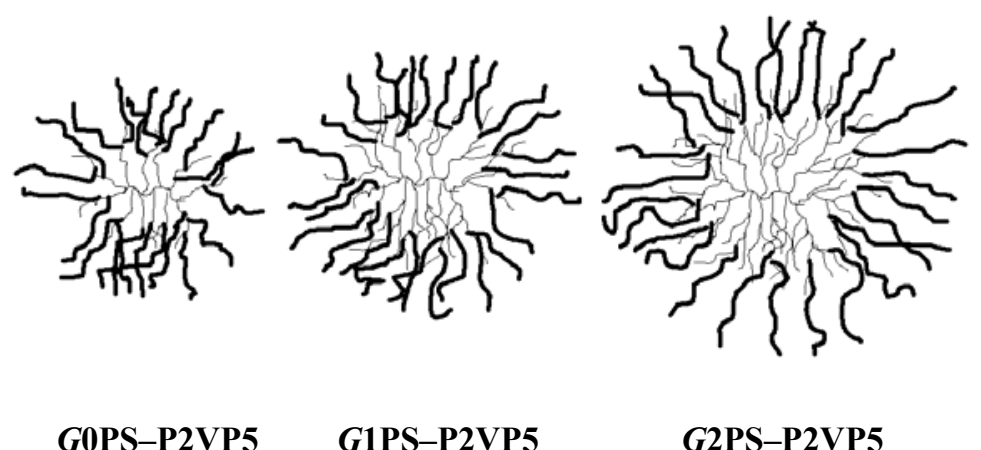

Figure 5.2. Structure of arborescent PS-P2VP5 copolymers of different generations.

The branching functionality of the core and the whole molecule, defined as the number of side chains added in the last grafting reaction, was calculated from the equation 


$$
f_{w}=\frac{M_{w}(G)-M_{w}(G-1)}{M_{w}^{b r}}
$$

where $M_{w}(G), M_{w}(G-1)$ and $M_{w}^{b r}$ are the absolute weight-average molecular weights obtained either from size exclusion chromatography using a multi-angle laser light scattering detector (SEC-MALLS) or batch-wise light scattering measurements for graft polymers of generation $G$, of the preceding generation, and of the side chains, respectively.

Table 5.1. Characteristics of Arborescent PS-g-P2VP Copolymers Used.

\begin{tabular}{|c|c|c|c|c|c|c|c|}
\hline \multirow{2}{*}{ Sample } & \multicolumn{2}{|c|}{ P2VP Side Chains } & \multicolumn{5}{|c|}{ Graft Copolymers } \\
\hline & $\mathrm{M}_{\mathrm{w}}{ }^{\mathrm{a}}$ & $\mathrm{M}_{\mathrm{w}} / \mathrm{M}_{\mathrm{n}}^{\mathrm{a}}$ & $\mathrm{M}_{\mathrm{w}}{ }^{\mathrm{a}}$ & $\mathrm{M}_{\mathrm{w}} / \mathrm{M}_{\mathrm{n}}{ }^{\mathrm{a}}$ & $f_{\mathrm{w}(\text { core })}$ & $f_{\mathrm{w}(\mathrm{copol})}$ & $\mathrm{Wt} \% \mathrm{PS}^{\mathrm{b}}$ \\
\hline G0PS-P2VP5-12 & 5100 & 1.06 & $4.7 \times 10^{5}$ & 1.08 & 11 & 82 & 12 \\
\hline G1PS-P2VP5-13 & 5200 & 1.08 & $3.7 \times 10^{6}$ & 1.07 & 84 & 630 & 13 \\
\hline G1PS-P2VP5-20 & 5200 & 1.09 & $2.4 \times 10^{6}$ & 1.09 & 84 & 370 & 20 \\
\hline G2PS-P2VP5-18 & 5300 & 1.07 & $2.2 \times 10^{7}$ & NA & 690 & 3400 & 18 \\
\hline G0PS-P2VP30-3 & 32000 & 1.07 & $2.2 \times 10^{6}$ & 1.07 & 11 & 67 & 3 \\
\hline G1PS-P2VP30-4 & 32000 & 1.07 & $1.1 \times 10^{7}$ & 1.08 & 84 & 330 & 4 \\
\hline
\end{tabular}

${ }^{\mathrm{a}}$ Absolute values determined from SEC-MALLS or laser light scattering measurements; G2PS-P2VP5-18 retained on column in SEC analysis; ${ }^{\mathrm{b}} \mathrm{PS}$ content determined by ${ }^{1} \mathrm{H}$ NMR spectroscopy.

5.4.1 Equilibrium Probe Uptake. One important parameter affecting probe uptake in solubilization experiments with block copolymers is their hydrophobic component content. In their study of poly(methacrylic acid)-block-polystyrene-block-poly(methacrylic acid) copolymer micelles, Cao et al. ${ }^{2 a}$ suggested that the much higher pyrene absorption capacity 
of their micelles ( $\kappa^{\mathrm{G}}=32 \mathrm{mg} / \mathrm{g}$ copolymer) as compared to the acrylamide-styrene block copolymer micelles $\left(\kappa^{\mathrm{G}}=1 \mathrm{mg} / \mathrm{g}\right.$ copolymer) of Dowling and Thomas ${ }^{14}$ was due to their higher polystyrene content. This effect was also reported by Hurter and Hatton, ${ }^{2 b}$ who found that the partition coefficient of naphthalene in solutions of Pluronic ${ }^{\circledR}$ copolymers varied as a function of the poly(ethylene oxide)-poly(propylene oxide) ratio. They further determined that for micelles obtained from copolymers of identical composition, copolymers with a higher molecular weight had higher solubilization capacities and partition coefficients. More recent studies ${ }^{9}$ have shown that pyrene uptake by dendrimers is a direct function of generation number, and therefore molecular size. It was argued that higher generation dendrimers were able to solubilize more pyrene specifically because they contained more microcavities for encapsulation.

The solubility of PyM in aqueous solutions was strongly enhanced by copolymers containing either short (P2VP5) or long (P2VP30) poly(2-vinylpyridine) side chains, however the effect was more pronounced for copolymers with shorter polyelectrolyte chains. The influence of copolymer structure and composition on solubilization is illustrated in Figure 5.3 for PyM with UV absorption spectra obtained for copolymer solutions (1.5 $\mathrm{mg} / \mathrm{mL}$ ) equilibrated with the probe. Similar measurements for $0.05 \mathrm{M} \mathrm{HCl}$ solutions saturated with PyM in the absence of copolymer yielded no detectable signal on the instrument used. The UV absorption spectra of Figure 5.3 indicate that PyM micellar loading for arborescent PS-P2VP copolymers correlates approximately with the PS content of the micelles, as solubilization increases with the PS content of the copolymers. Based on the above discussion regarding the influence of size, micelles of G1PS-P2VP5-13, with a PS content comparable to G0PS-P2VP5-12 but larger in size, would be expected to have a 
higher solubilization capacity. The opposite was actually observed experimentally, as the UV absorbance for G0PS-P2VP5-12 in Figure 5.3 is significantly higher than for G1PS-P2VP513. One important characteristic of arborescent polymers is increased segmental density for higher generations, because of the slow increase in their radius of gyration relative to their hydrodynamic radius. ${ }^{15}$ This is partly a consequence of the larger number of branching points in G1PS-P2VP5-13 $\left(f_{\mathrm{w}}=630\right)$ than in G0PS-P2VP5-12 $\left(f_{\mathrm{w}}=82\right)$. The solubilization of hydrophobic probes in the non-polar PS core may become more difficult due to increased segmental density in copolymers with a higher branching functionality $f_{\mathrm{w}}$. Consequently, lower generation arborescent copolymers are likely to solubilize a larger amount of hydrophobic guest compounds than higher generation copolymers of similar composition.

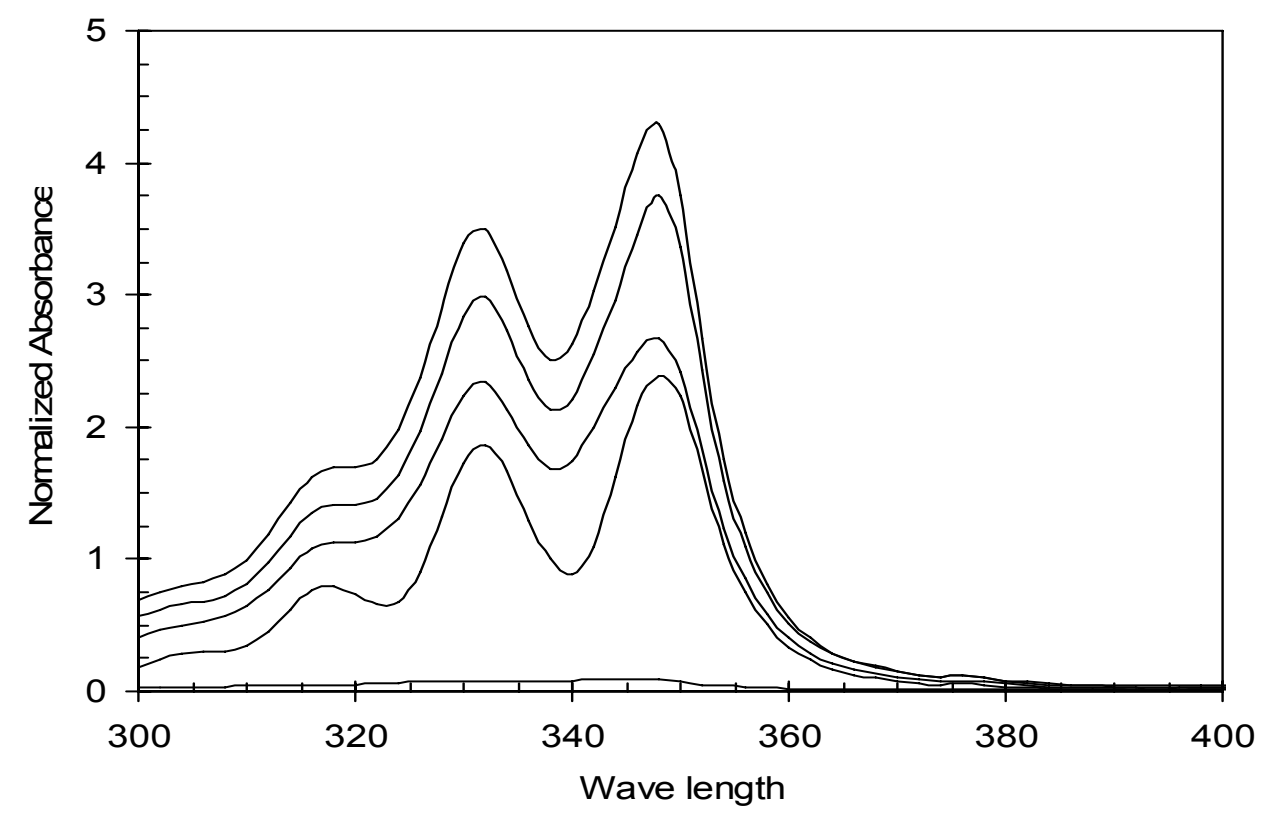

Figure 5.3. UV equilibrium absorption spectra for PyM solubilized by arborescent copolymer samples. The curves, from top to bottom, are for G1PS-P2VP5-20, G2PSP2VP5-18, G0PS-P2VP5-12, G1PS-P2VP5-13, and G0PS-P2VP30-3. 
For copolymers with long branches (P2VP30), solubility enhancement of PyM was minimal (Figure 5.3). Though the longer P2VP chains of the P2VP30 copolymers might have hindered the diffusion of PyM molecules to the PS core, the much lower polystyrene content of the P2VP30 copolymers seems the main reason for lower PyM uptake. Because of their very low absorption capacity, investigations of copolymers based on P2VP30 side chains were more limited.

5.4.2 Micellar Uptake Kinetics. The kinetics of the absorption process were investigated by monitoring PyM uptake as a function of time. Equilibration was assumed when a plateau was reached in the absorbance versus time plot (Figure 5.4). Composition and

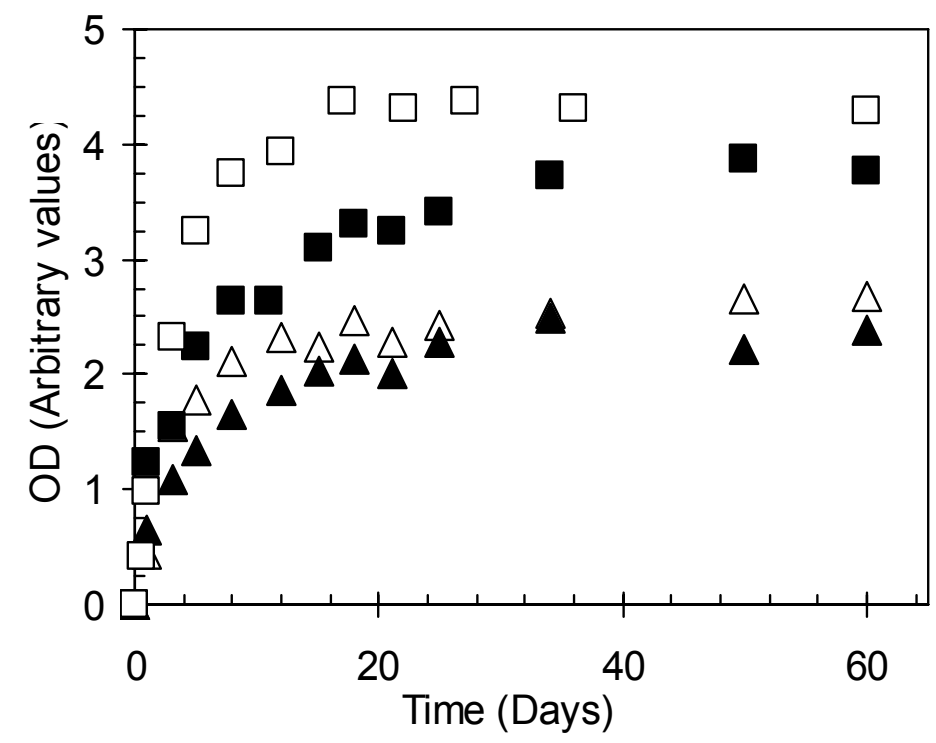

Figure 5.4. Time dependence of PyM uptake by arborescent micelles: $(\Delta)$ G0PS-P2VP5-12; (ム) G1PS-P2VP5-13; () G1PS-P2VP5-20; (ロ) G2PS-P2VP5-18. 
branching functionality have a significant influence on the equilibration time of P2VP5 copolymer samples: Copolymers with high PS contents and $f_{\mathrm{w}}$ have longer equilibration times (25-35 days) than copolymers with a lower PS content and a low $f_{\mathrm{w}}$ (8-20 days). Quantitative information of the rate of solubilization was obtained by using the first-order kinetic equation ${ }^{16}$

$$
[P y M]_{t}=[P y M]_{e q}(1-\exp (-k t))
$$

to fit our data, where $[P y M]_{e q}$ is the equilibrium concentration of PyM in the micelles, and $[P y M]_{t}$ is the concentration of PyM in the micelle at time $t$. With this expression, it was possible to quantify the uptake rate in terms of the rate constant $k$.

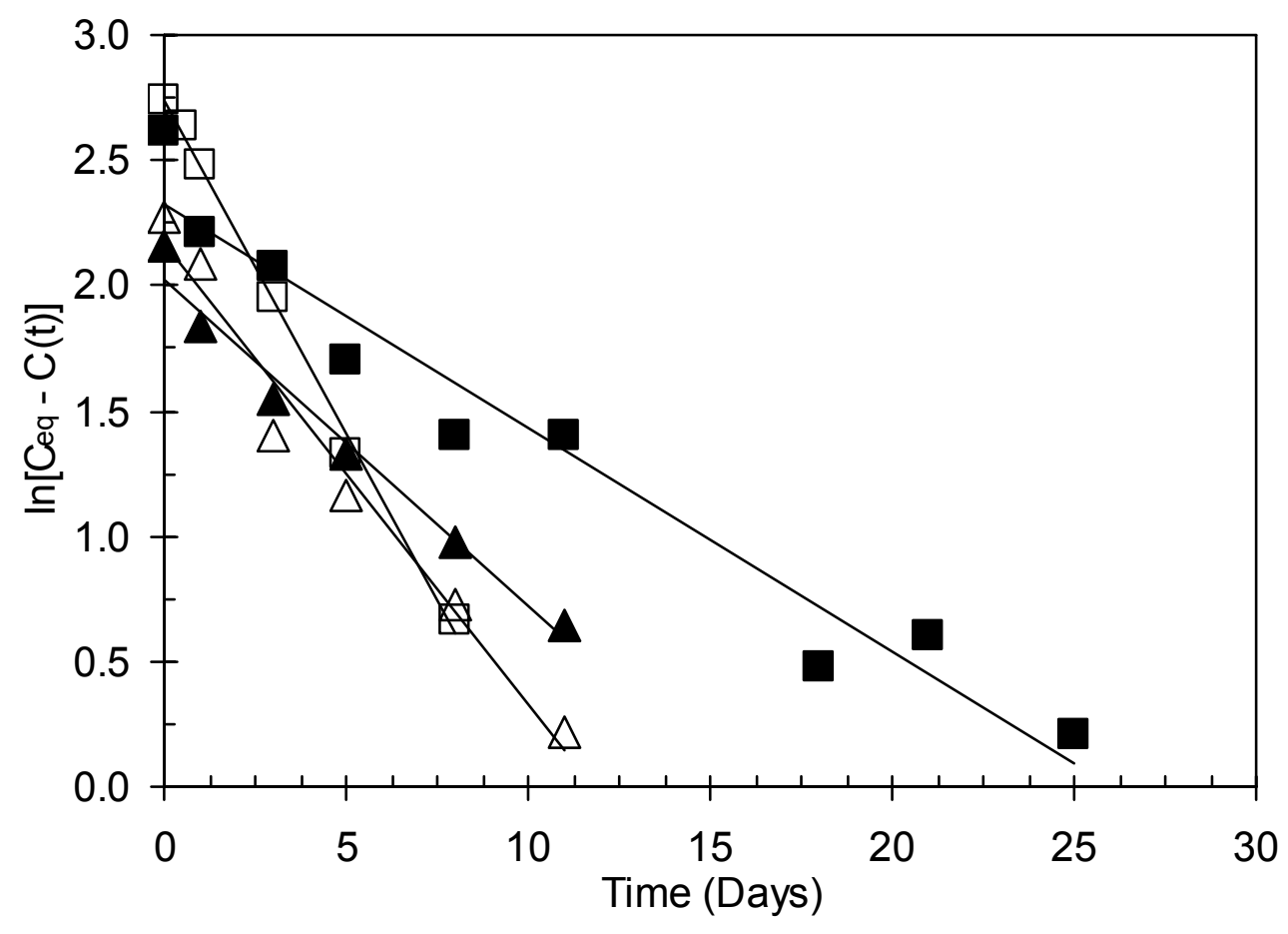

Figure 5.5. Analysis of micellar uptake kinetics. The following rate constants were obtained: ( $\Delta$ ) G0PS-P2VP5-12, $k=(0.18 \pm 0.01) \mathrm{d}^{-1} ;(\boldsymbol{\Delta})$ G1PS-P2VP5-13, $k=(0.13 \pm 0.01) \mathrm{d}^{-1} ;(\square)$ G1PS-P2VP5-20, $k=(0.266 \pm 0.006) \mathrm{d}^{-1} ;(\boldsymbol{\bullet})$ G2PS-P2VP5-18, $k=(0.089 \pm 0.007) \mathrm{d}^{-1}$. 
Figure 5.5 is a plot of $\ln \left([P y M]_{e q}-[P y M]_{t}\right)$ versus time, corresponding to the logarithmic form of Equation 5.4. The rate of PyM uptake depends on both branching functionality and copolymer composition, as seen from the different rate constants (slopes) observed for the respective copolymers. The lower solubilization rates observed for higher generation arborescent copolymers are attributed to the higher branching functionality of the micelle core making it more difficult for the probe to diffuse inside, as outlined above. If this hypothesis is valid, decreasing the branching functionality of the copolymer micelles should enhance the uptake rate. The branching functionality of a copolymer sample was decreased by lowering the acetylation level of the G1PS substrate used in the synthesis. Samples G1PS-P2VP5-13 and G1PS-P2VP5-20 were synthesized from the same polystyrene core, using acetylation levels of $25 \mathrm{~mol} \%$ and $8 \mathrm{~mol} \%$, respectively. This explains the lower branching functionality and higher PS content obtained for G1PS-P2VP5-20. As can be seen from their respective rate plots (Figure 5.5), G1PS-P2VP5-20 has a significantly higher rate constant $\left(k=0.27 \mathrm{~d}^{-1}\right)$ than G1PS-P2VP-13 $\left(k=0.13 \mathrm{~d}^{-1}\right)$. The range of solubilization rate constants obtained, $k=0.09-0.27 \mathrm{~d}^{-1}$ for the different samples, suggests that it is possible to control the solubilization behavior of the micelles with proper design of the copolymer architecture.

\subsubsection{Determination of Partition Coefficient and Solubilization Capacity. The} partition of a hydrophobic probe between the bulk aqueous phase and the micelles can be characterized by the partition coefficient at equilibrium, $K_{\mathrm{w}}$, and the solubilization capacity, $\kappa^{\mathrm{G}}$. Partition experiments were conducted to determine the influence of polymer as well as probe structure on both $K_{\mathrm{w}}$ and $\kappa^{\mathrm{G}}$. 
Preliminary measurements to determine the solubility of PyM in acidified ( $0.05 \mathrm{M}$ $\mathrm{HCl}$ ) water showed that it was about 5 times lower than in neutral water. Consequently solubility limits reported for the probes in the literature could not be used for $\mathrm{S}_{\mathrm{s}}$ in Equation 2, but rather had to be determined under the conditions used as described in the Experimental Section. The aqueous solubility of PyM and Py estimated by fluorescence is $1.8 \times 10^{-6} \mathrm{M}$ and $9.8 \times 10^{-8} \mathrm{M}$ for PyM and Py, respectively. The solubility of Nap $\left(9.3 \times 10^{-5} \mathrm{M}\right), 1-\mathrm{NapBr}$ $\left(2.6 \times 10^{-5} \mathrm{M}\right)$, NapMe $\left(6.4 \times 10^{-5} \mathrm{M}\right)$ and Phe $\left(4.9 \times 10^{-5} \mathrm{M}\right)$ in aqueous $0.05 \mathrm{M} \mathrm{HCl}$ was sufficiently high to determine their concentration from UV absorption measurements. Because of instrumental limitations, the solubility of $\mathrm{An}$ in $0.05 \mathrm{M} \mathrm{HCl}$ could not be determined. A literature value $\left(2.8 \times 10^{-7} \mathrm{M}\right)^{17}$ for the solubility in pure water was used in this case, so the $K_{\mathrm{w}}$ values determined for An may be underestimated. Because of important differences in the behavior of highly hydrophobic (Py, PyM, An, Phe) and less hydrophobic probes (Nap, NapBr, NapMe), the results obtained for each series of compounds will be discussed separately.

5.4.4 Solubilization of Highly Hydrophobic Probes. From Table 5.2 it is clear that the partition coefficient for PyM increases with the PS content of the copolymers, with values ranging from $0.9-1.7 \times 10^{5}$. For Py, a more hydrophobic probe than PyM, the partition coefficient is even higher, ranging from $0.3-1.2 \times 10^{6}$. An and Phe also have higher $K_{\mathrm{w}}$ values for copolymers with a higher PS content. The same trend has been observed in other studies using block copolymer micelles. , $^{2,18,19}$

The octanol-water partition coefficient $\left(K_{\mathrm{ow}}\right)$ and the boiling point $(\mathrm{bp})$ of small molecule hydrophobes have been correlated successfully with $K_{\mathrm{w}}$ values for block copolymer micelles in the past. ${ }^{1 b, 2 b, 20}$ These properties are reported in Table 5.3 for the probes used in 
Table 5.2. Partition Coefficient $K_{\mathrm{w}}$ for PAHs.

\begin{tabular}{lccccccc}
\hline Sample & $K_{w}$ PyM & $K_{w}$ Py & $K_{w}$ An & $K_{w}$ Phe & $K_{w}$ Nap & $K_{w}$ NapMe & $K_{w}$ NapBr \\
\hline G0PS-P2VP5-12 & $9.6 \times 10^{4}$ & $3.7 \times 10^{5}$ & $1.2 \times 10^{5}$ & $1.7 \times 10^{5}$ & $8.7 \times 10^{2}$ & $2.2 \times 10^{4}$ & $5.0 \times 10^{3}$ \\
G1PS-P2VP5-13 & $8.9 \times 10^{4}$ & $2.8 \times 10^{5}$ & $8.1 \times 10^{4}$ & $1.8 \times 10^{5}$ & $9.5 \times 10^{2}$ & $2.3 \times 10^{4}$ & $9.7 \times 10^{3}$ \\
G1PS-P2VP5-20 & $1.7 \times 10^{5}$ & $1.2 \times 10^{6}$ & & & & $3.1 \times 10^{4}$ & \\
G2PS-P2VP5-18 & $1.5 \times 10^{5}$ & $7.0 \times 10^{5}$ & $3.1 \times 10^{5}$ & $4.1 \times 10^{5}$ & $2.1 \times 10^{3}$ & $2.9 \times 10^{4}$ & $1.5 \times 10^{4}$ \\
\hline
\end{tabular}

Abbreviations used: PyM (1-Pyrenemethanol), Py (Pyrene), An (Anthracene), Phe
(Phenanthrene), Nap (Naphthalene), NapMe (1-Methylnaphthalene), NapBr (1-
Bromonapthalene).

the present study. The partition coefficients of Table 5.2 indeed increase with probe hydrophobicity in the series $\mathrm{PyM}<\mathrm{Phe} \approx \mathrm{An}<\mathrm{Py}$ as expected. Almgren et al. ${ }^{20}$ have successfully correlated the partition coefficient for a series of arenes in alkyltrimethylammonium bromide and sodium alkyl sulfate micelles to their boiling points. For both types of ionic micelles, a linear relationship was obtained between the boiling point and the partition coefficient of the arenes. The correlation is likewise successful in terms of boiling points of the probes in the limited series $\mathrm{Phe}=\mathrm{An}<\mathrm{Py}$.

In contrast, no clear trends are observed when trying to correlate the solubilization capacity $\kappa^{\mathrm{G}}$ (Table 5.4) to either $K_{\mathrm{ow}}$ values or boiling points. For example, An and Phe are tricyclic isomers of very similar hydrophobicity, as indicated by their $\log K_{\text {ow }}$ values of 4.56 and 4.52, respectively (Table 5.3). These two compounds might therefore be expected to have similar $\kappa^{\mathrm{G}}$ values. While $K_{\mathrm{w}}$ is similar for both molecules, the solubilization capacity for Phe is much higher than for An. Correlation of $\kappa^{\mathrm{G}}$ with the boiling point of the probes used is likewise unsuccessful. Molecular size arguments have been used in the past to explain the 
Table 5.3. Physical Properties of PAHs. ${ }^{17}$

\begin{tabular}{lll}
\hline & $\log K_{\text {ow }}$ & $\mathrm{Bp}\left({ }^{\circ} \mathrm{C}\right)$ \\
\hline Naphthalene & 3.34 & 217.9 \\
1-Methylnaphthalene & 3.87 & 244.7 \\
1-Bromonaphthalene & 4.35 & 281 \\
Anthracene & 4.56 & 339.9 \\
Phenanthrene & 4.52 & 340 \\
Pyrene & 5.08 & 404 \\
1-Pyrenemethanol & 3.99 & dec \\
\hline
\end{tabular}

decreasing solubilization capacity of Pluronic ${ }^{\circledR}$ block copolymer micelles for hydrophobes in the series Nap, Phe, Py. ${ }^{2 b}$ As the number of benzene rings per molecule is increased, solubilization should become energetically more difficult because of the larger volume of the solute molecules. On that basis, higher $\kappa^{\mathrm{G}}$ values are expected for the solubilization of An and Phe by arborescent micelles than for Py and PyM. Unfortunately the $\kappa^{\mathrm{G}}$ values observed for arborescent copolymers are larger for Py than for An, and larger for PyM than for An and Phe. The lack of trends in the current investigation suggests that molecular size, hydrophobicity, and solute-solute interactions are not dominant factors determining (at least individually) the solubilization capacity of arborescent micelles. 
Table 5.4. Solubilization Capacity $\kappa^{G}$ (mg probe/g polymer) for PAHs.

\begin{tabular}{lccccccc}
\hline Sample & $\kappa^{G}$ PyM & $\kappa^{G} \mathrm{Py}$ & $\kappa^{G}$ An & $\kappa^{G}$ Phe & $\kappa^{G}$ Nap & $\kappa^{G}$ NapMe & $\kappa^{G}$ NapBr \\
\hline G0PS-P2VP5-12 & 8.7 & 2.5 & 0.3 & 4.9 & 10 & 35 & 27 \\
G1PS-P2VP5-13 & 8.1 & 1.8 & 0.2 & 5.5 & 11 & 39 & 53 \\
G2PS-P2VP5-18 & 14 & 4.6 & 0.8 & 12 & 25 & 99 & 81 \\
G1PS-P2VP5-20 & 15 & 8.0 & & & & 120 & \\
\hline
\end{tabular}

5.4.5 Solubilization of Naphthalene and Derivatives. Comparison of the $K_{\mathrm{w}}$ (Table 5.2) and $\kappa^{\mathrm{G}}$ values (Table 5.4) obtained for the different copolymers reveals significant differences in behavior for Nap and its derivatives relative to the larger size probes. Nap is the least hydrophobic of all 7 probes investigated, and indeed has the lowest overall $K_{\mathrm{w}}$ values in the series. NapMe, with the second lowest $\log K_{\mathrm{ow}}$ in the series (albeit close to PyM), ranks third for $K_{\mathrm{w}}$, behind NapBr. Finally NapBr, with the fourth lowest $\log K_{\mathrm{ow}}$ value, ranks second lowest in terms of $K_{\mathrm{w}}$.

A striking difference is observed for Nap and its derivatives in terms of solubilization capacities (Table 5.4): The $\kappa^{\mathrm{G}}$ values for NapMe and NapBr, in particular, are much higher than for the other probes. The influence of branching functionality and side chain molecular weight on the physical properties of arborescent polystyrenes has been the object of a number of investigations. ${ }^{21}$ Arborescent polystyrene molecules resemble nanonetworks in that they swell in good solvents. Once in the micelles, a liquid hydrophobic probe could behave as a solvent and swell the core to some extent. Naphthalene, NapMe, and $\mathrm{NapBr}$ were investigated as probes of lower hydrophobicity than the tri- and tetracyclic PAHs discussed so far. Two of these (NapMe and NapBr) are liquids. It may be tempting to attribute the 
much higher $\kappa^{\mathrm{G}}$ values observed for these compounds solely to swelling of the PS micelle cores by the liquid probes. In the following discussion, it will be demonstrated that while core swelling may be significant, it is likely not the only reason for the higher sorption of naphthalene probes.

5.4.6 Location of the Probes in the Micelles. It has been suggested ${ }^{13}$ that the morphology of micelles includes three regions: the hydrophobic core, the hydrophilic shell (corona), and the so-called palisade, which is the interfacial region between the core and the shell. For a more complete understanding of a micellar system, it is worthwhile to determine where the molecular solute is located inside the micelles. Depending on the relative hydrophobicity of a probe and the copolymer components, a probe can be located in one or more of the three regions mentioned. Highly hydrophobic solutes are typically solubilized in the micellar core, while semipolar and polar solutes can be solubilized in the palisade and/or the corona. It is well established that increasing the hydrophobic phase content of micellar systems greatly enhances the solubilization capacity and partition coefficient for Py, a highly hydrophobic probe preferentially solubilized in the core. ${ }^{2 a, b, 14}$ In contrast, the solubilization of semipolar solutes remains almost unaffected by hydrophobic phase content. ${ }^{13,22}$ In such situations, the semipolar solutes are said to be associated with either the palisade or the hydrophilic corona. For relatively polar solutes, solubilization may even decrease with increasing hydrophobic phase content, due to preferential sorption in the corona. ${ }^{23}$

A plot of the partition coefficient $K_{\mathrm{w}}$ versus PS weight fraction is used in Figure 5.6 to compare the behavior of three of the probes used (Py, PyM, and NapMe). The partition coefficient for Py increases sharply as the PS content of the copolymer increases, in analogy to other micellar systems. This suggests that Py is located predominantly in the core of the 
micelles. In contrast, the relative increase in $K_{\mathrm{w}}$ is less significant for PyM and minimal for NapMe. The insensitivity of $K_{\mathrm{w}}$ to composition for PyM and NapMe could be explained by preferential partition of these more polar compounds to the palisade and/or corona regions of the micelles.

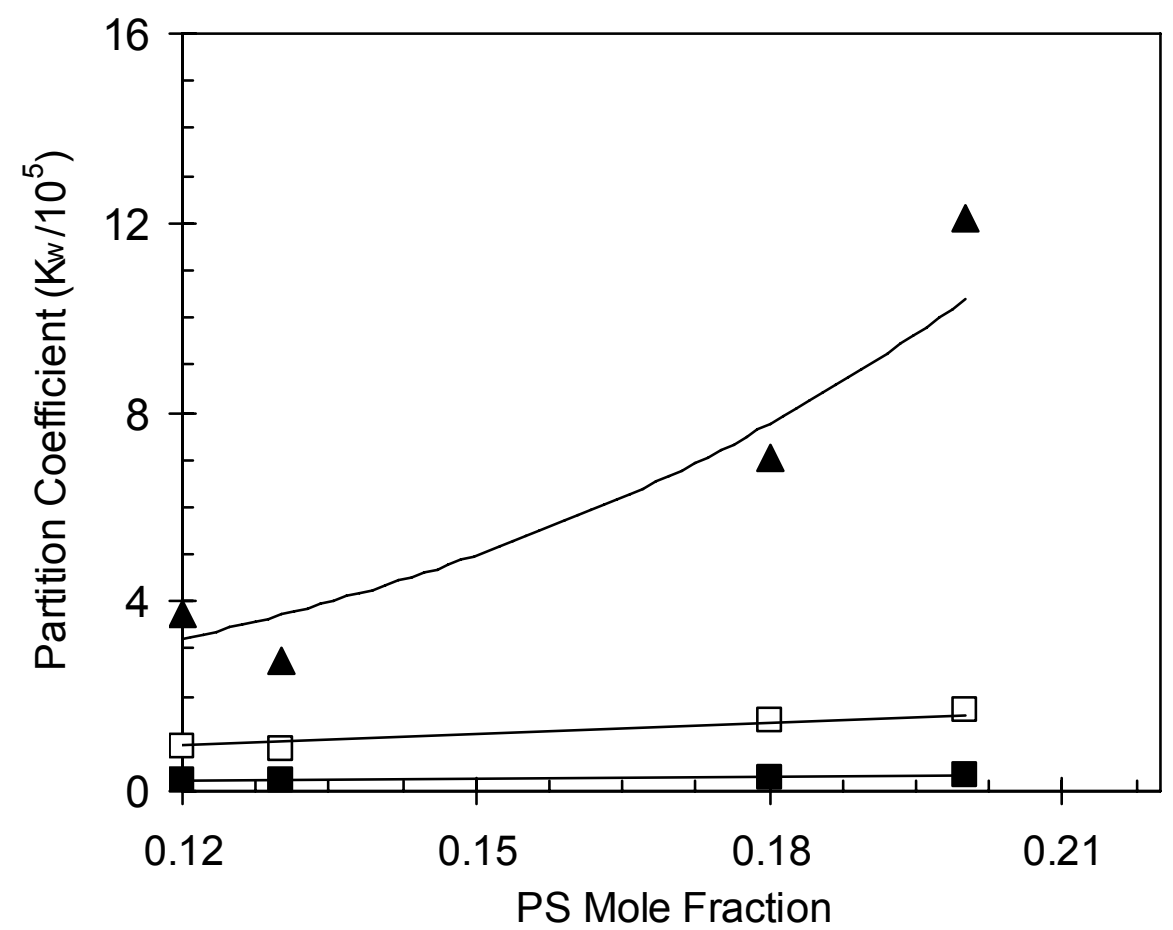

Figure 5.6. Composition dependence of partition coefficient for ( $\mathbf{\Delta})$ Py, ( $\square$ ) PyM, and ( $\mathbf{\square})$ NapMe.

If the corona region contributes significantly to the solubilization process, copolymers in the P2VP30 series, with a very low PS content, should be able to solubilize large amounts of the most polar compounds (Nap and derivatives). For both G0PS-P2VP30-3 and G1PS$\mathrm{P} 2 \mathrm{VP} 30-4, \kappa^{\mathrm{G}}=11 \mathrm{mg} / \mathrm{g}$ was determined using Nap as probe. The same copolymers yielded $\kappa^{\mathrm{G}}=17$ and $21 \mathrm{mg} / \mathrm{g}$, respectively, for NapBr. In comparison, $\kappa^{\mathrm{G}}=0.08 \mathrm{mg} / \mathrm{g}$ for PyM in 
G0PS-P2VP30-3, and the amount of Py solubilized by the same compound was too low to be detected. Based on these results, the high $\kappa^{\mathrm{G}}$ values observed for the Nap derivatives is attributable at least in part to non-selective sorption of the probes, beyond the core swelling effect already discussed: A significant portion of these compounds is located in the corona and/or palisade regions of the P2VP-rich micelles, rather than preferentially in the core like pyrene. The low sorption capacity observed for PyM relative to the naphthalene derivatives is consistent with its location in a restricted volume outside the core, i.e. the palisade region.

5.4.7 Additional Evidence in Support of Selective Sorption. The intensity of fluorescence emission observed was compared for PyM in $0.05 \mathrm{M} \mathrm{HCl}$, in the presence of linear P2VP, and with the arborescent copolymer micelles. The ratio of intensities determined for the first vibronic band $I_{1}(373 \mathrm{~nm})$ of PyM in acidified water, and in acidified solutions of linear P2VP and G0PS-P2VP5-12 is 1:4:75. The large intensity difference observed between linear P2VP and G0PS-P2VP5 confirms that the solubility of PyM in ionized P2VP is very low. The fraction of PyM associated strictly with the P2VP shell of arborescent PS-P2VP5 copolymers is therefore considered negligible under the conditions used, and these probes must be located predominantly in the palisade region.

A red shift in the UV absorption spectrum was observed for PyM, as $\lambda_{\max }$ shifted from $342 \mathrm{~nm}$ for the P2VP30 copolymers to $348 \mathrm{~nm}$ for the P2VP5 copolymers (Figure 5.7). A study of $\mathrm{PyM}^{24}$ has shown that in a hydrophobic environment such as PS, $\lambda_{\max }$ is higher (346 nm) than in polar environments $(339 \mathrm{~nm}$ for $\mathrm{MeOH})$. Red shifts have also been observed for the fluorescence excitation spectrum of pyrene $\mathrm{e}^{3 \mathrm{c}, 18,25}$ and the UV absorption spectrum of a cyclic disulfide containing pyrene residues. ${ }^{26}$ These effects have been linked systematically to the transfer of Py from water to a more hydrophobic environment. The 


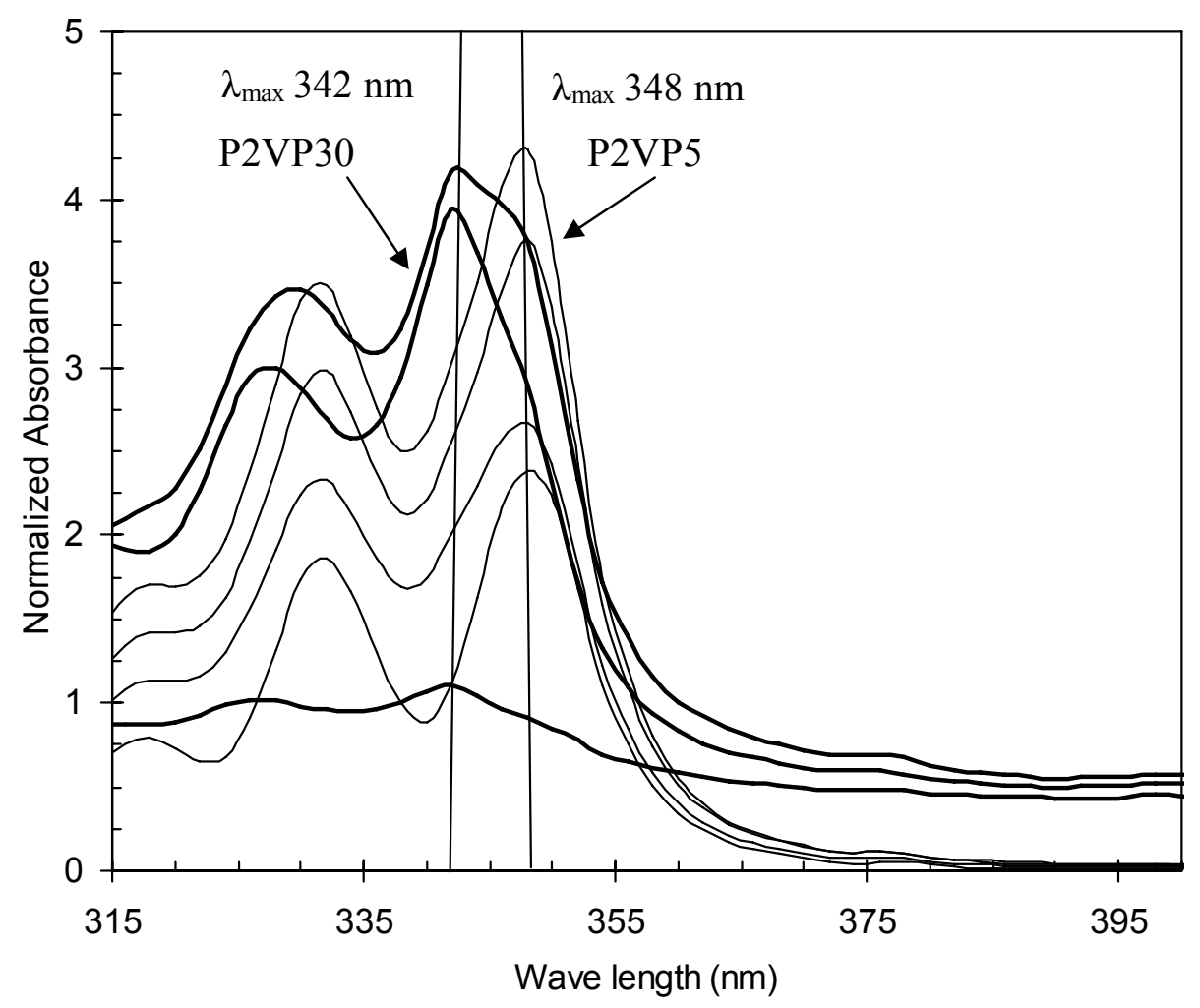

Figure 5.7. Red shift of UV absorbance of PyM in P2VP30 and P2VP5 samples. $\lambda_{\max }=342$ nm for P2VP30 samples, 348 nm for P2VP5 samples; spectra for P2VP30 samples have been magnified for clarity, hence the shift in their baselines.

change in $\lambda_{\max }$ observed for PyM in the present study confirms that the small amount of PyM solubilized by the long-branch (P2VP30) copolymers resides mainly in a more hydrophilic (P2VP-rich) environment, corresponding to the P2VP hydrophilic shell, the distortion of the P2VP30 spectra around $\lambda=348 \mathrm{~nm}$ resulting from core solubilized PyM. PyM solubilized by short-branch (P2VP5) copolymers resides mostly in a more hydrophobic region such as the core and the palisade.

Additional evidence for partitioning of the hydrophobic probes within the micelles was obtained from steady-state fluorescence measurements using Py, since it is a widely used 
polarity-sensitive probe. The intensity ratio of first vibronic band $I_{1}(373 \mathrm{~nm})$ to the third vibronic band $I_{3}(383 \mathrm{~nm})$ undergoes significant changes with solvent polarity, as a result of coupling of the electronic and vibronic states, and can serve to monitor the polarity of the microenvironment surrounding the probe. ${ }^{27}$ For G2PS-P2VP5-18, an $I_{1} / I_{3}$ value of $1.03 \pm$ 0.01 was obtained, and for G1PS-P2VP5-20, the $I_{1} / I_{3}$ value was $0.97 \pm 0.03$. Literature values for $I_{1} / I_{3}$ in water range from $1.6-1.96 .{ }^{27 a, 28}$ The ratio decreases for increasing microenvironment hydrophobicity, to 0.95 in PS films, for example. ${ }^{18}$ The $I_{1} / I_{3}$ ratios obtained for the arborescent copolymers are consistent with the concentration of the Py probes in the PS core of the micelles.

Finally, it is interesting to note the absence of the broad PyM and Py excimer emission peaks for all PS-P2VP copolymer micelle solutions investigated, even at very high probe uptake. Excimer formation can result from a dynamic process, whereby an electronically excited pyrene diffuses to encounter a pyrene molecule in its ground state, or from a static process involving the excitation of preassociated ground state pyrene molecules. It was suggested that diffusion-controlled excimer formation inside a hydrophobic domain depends on the microviscosity of the micelles. ${ }^{29}$ According to this interpretation, the fact that no excimer peaks are observed for Py and PyM is consistent with a very dense (collapsed) core structure making it almost impossible for two closely located probe molecules to diffusionally encounter during the lifetime of the excited molecule. Though excimer peaks are absent, the possibility of probe aggregation cannot be excluded. There have been reports of preassociation of pyrene and its derivatives in molecular assemblies. ${ }^{24,30}$ In some instances, extensive self-quenching due to the low quantum yield of these aggregates has been reported. ${ }^{30,31}$ In such situations, the intensity of the broad peak with maximum at 485 
$\mathrm{nm}$ associated with excimer formation observed in the fluorescence emission spectrum decreases or even disappears. The peak-to-valley ratio for the UV absorption spectrum of the most intense band of Py derivatives (ca. $348 \mathrm{~nm}$ ) to that of the adjacent minimum at shorter wavelength (ca. $338 \mathrm{~nm}$ ) has been linked to preassociation in pyrene-labeled polymers. ${ }^{31}$ This method is convenient to detect preassociation when no excimer emission peak is observed. A peak-to-valley ratio greater than 3 indicates the absence of aggregation; the ratio decreases for increasing preassociation. From the absorption spectra of PyM (Figure 5.3) and Py (not shown), the peak-to-valley ratio ranges from 1.6-2.86 for all micellar solutions, indicating that some of the probe molecules are aggregated in the ground state.

The preassociation of pyrene labeled-polymers is sometimes accompanied by a hypochromic effect (decrease in molar extinction coefficient). ${ }^{32}$ From Figure 5.3, PyM in G1PS-P2VP5-13 has the highest peak-to-valley ratio of $\sim 2.69$, and the hypochromic effect is therefore likely to be minimal (least association) when compared to PyM in the other copolymer solutions. If this is true the solubilization capacity of G1PS-P2VP5-13\% might be underestimated, since the same molar extinction coefficient $\left(35943 \mathrm{~mol}^{-1} \mathrm{~cm}^{-1}, \lambda_{\max }=346\right.$ in toluene) was used to analyze all the micellar solutions. This observation has no bearing on the fact that for different generation copolymers with similar composition, the lower generation copolymers solubilize more probes, however. Py has similar peak-to-valley ratios for all copolymer solutions used, and the solubilization capacity for G0PS-P2VP5-12 is greater than for G1PS-P2VP-13. This is also observed for anthracene. 


\subsection{Conclusions}

Partitioning of hydrophobic solutes between arborescent polystyrene-graft-poly(2vinylpyridine) unimolecular micelles and water depends on both the structure of the micelles and the nature of the probes. Arborescent copolymers with a high PS content are far better sorbents than samples with a low PS content. The structure of the solute also has a large effect on the partition coefficients and micelle loadings observed. Pyrene has partition coefficients reaching $\sim 10^{6}$, however probes with a lower hydrophobicity give higher micelle loadings. The generation number and hydrophobic content of the copolymers have a significant influence on both uptake capacity and kinetics. For samples of the P2VP5 series the partition coefficient increases rapidly with the PS mole fraction of the copolymers for highly hydrophobic probes, while only a minor increase is observed for probes of lower hydrophobicity. For copolymers of different generations with similar PS contents however, the solubilization capacity is higher for the lower generation copolymers. Copolymers with a higher branching functionality are also characterized by lower probe solubilization rates. These results are consistent with the segmental density of arborescent polymer molecules increasing for higher generations, as determined in previous studies.

\subsection{Acknowledgements}

We are grateful for the financial support of the Natural Sciences and Engineering Research Council of Canada (NSERC) and the International Council for Canadian Studies (ICCS) for this work. We are also indebted to Prof. Jean Duhamel for useful discussions and for providing access to the fluorescence equipment. 


\subsection{References}

1. (a) Yokoyama, M.; Kwon, G. S.; Okano, T.; Sakurai, Y.; Seto, T.; Kataoka, K. Bioconjugate Chem. 1992, 3, 295. (b) Haulbrook, W. R.; Feerer, J. L.; Hatton, T. A.; Tester, J. W. Environ. Sci. Technol. 1993, 27, 2783. (c) Quintana, J. R.; Salazar, R. A.; Katime, I. Macromolecules, 1994, 27, 665. (d) Kwon, G. S.; Okano, T. Adv. Drug Delivery Rev. 1996, 21, 107. (e) Richardson, M. F.; Armentrout, R. S.; McCormick, C. L. J. Appl. Polym. Sci. 1999, 74, 2290. (f) van Domeselaar, G. H.; Kwon, G. S.; Andrew, L. C.; Wishart, D. S. Colloids Surf. B: Biointerfaces 2003, 30, 323. (g) Glass, R.; Arnold, M.; Blümmel, J.; Küller, A.; Möller, M.; Spatz, J. P. Adv. Funct. Mater. 2003, 13, 569 .

2. (a) Cao, T.; Munk, P.; Ramireddy, C.; Tuzar, Z.; Webber, S. E. Macromolecules 1991, 24, 6300. (b) Hurter, P. N.; Hatton, T. A. Langmuir 1992, 8, 1291. (c) Gadelle, F.; Koros, W. J.; Schechter, R. S. Macromolecules 1995, 28, 4883. (d) Zhao, J.; Allen, C.; Eisenberg, A. Macromolecules 1997, 30, 7143.

3. (a) Gitsov, I.; Fréchet, J. M. J. Macromolecules 1993, 26, 6536. (b) Fréchet, J. M. J; Gitsov, I. Macromol. Symp. 1995, 98, 441. (c) Chang, Y.; Kwon, Y. C.; Lee, S. C.; Kim, C. Macromolecules 2000, 33, 4496. (d) Chang, Y.; Kim, C. J. Polym. Sci., Part A: Polym Chem. 2001, 39, 918.

4. (a) Fréchet, J. M. J. Science 1994, 263, 1710. (b) Stevelmans, S.; van Hest, J. C. M.; Jansen, J. F. G. A.; van Boxtel, D. A. F. J.; de Brabander-van den Berg, E. M. M.; Meijer, E. W. J. Am. Chem. Soc. 1996, 118, 7398. (c) Twyman, L. J.; Beezer, A. E.; Esfand, R.; Hardy, M. J.; Mitchell, J. C. Tetrahedron Lett. 1999, 40, 1743. (d) Liu, M.; Fréchet, J. M. J. Pharm. Sci. Technol. To. 1999, 2, 393. (e) de Groot, D.; de Waal, B. F. 
M.; Reek, J. N. H.; Schenning, A. P. H. J.; Kamer, P. C. J.; Meijer, E. W.; van Leeuwen, P. W. N. M. J. Am. Chem. Soc. 2001, 123, 8453. (f) Dykes, G. M. J. Chem. Technol. Biotechnol. 2001, 76, 903. (g) Turro, N. J.; Chen, W.; Ottaviani, M. F. In Dendrimers and other Dendritic Polymers; Fréchet, J. M. J and Tomalia, D. A., Eds.; Wiley: West Sussex, 2001; Chapter 13. (h) Newkome, G. R.; Moorefield, C. N.; Vögtle, F. Dendrimers and Dendrons: Concepts, Syntheses, Applications; Wiley-VCH: New York, 2001. (i) Weener, J.-W.; Baars, M. W. P. L.; Meijer, E. W. In Dendrimers and other Dendritic Polymers; Fréchet, J. M. J and Tomalia, D. A., Eds.; Wiley: West Sussex, 2001; Chapter 16. (j) Beezer, A. E.; King, A. S. H.; Martin, I. K.; Mitchel, J. C.; Twyman, L. J.; Wain, C. F. Tetrahedron 2003, 59, 3873.(k) Niwa, M.; Higashizaki, T.; Higashi, N. Tetrahedron 2003, 59, 4011.

5. (a) Kwon, G.; Suwa, S.; Yokoyama, M.; Okano, T.; Sakurai, Y.; Kataoka, K. J. Controlled Release 1994, 29, 17. (b) Yuan, F.; Dellian, M.; Fukumura, D.; Leunig, M.; Berk, D. A.; Torchilin, V. P.; Jain, R. K. Cancer Res. 1995, 55, 3752. (c) Kim, S. Y.; Shin, I. G.; Lee, Y. M.; Cho, C. S..; Sung, Y. K. J. Controlled Release 1998, 51, 13. (d) Kong, G.; Braun, R. D.; Dewhirst, M. W. Cancer Res. 2000, 60, 4440. (e) Ducan, R.; Izzo, L. Adv. Drug Delivery Rev. 2005, 57, 2215.

6. For a recent review on the synthesis and properties of arborescent and other dendrigraft polymers, see Teertstra, S. J.; Gauthier, M. Prog. Polym. Sci. 2004, 29, 277.

7. Gauthier, M.; Li, J.; Dockendorff, J. Macromolecules 2003, 36, 2642.

8. Kwon, G. S.; Naito, M.; Kataoka, K.; Yokoyama, M.; Sakurai, Y.; Okano, T. Colloids Surfaces B: Biointerfaces 1994, 2, 429. 
9. (a) Pistolis, G.; Malliaris, A; Paleos, C. M.; Tsiourvas, D. Langmuir 1997, 13, 5870.

(b) Liu, M.; Kono, K.; Fréchet, J. M. J. J. Controlled Release 2000, 65, 121

10. Phoon, C. L.; Higgins, J. S.; Burchard, W.; Peiffer, D. G. Macromol. Rep. 1992 A29 (Suppl. 2), 179.

11. Astafieva, I.; Zhong, X. F.; Eisenberg, A. Macromolecules 1993, 26, 7339.

12. Kee, R. A.; Gauthier, M. Macromolecules 2002, 35, 6526.

13. Yalkowsky, H. S. Solubility and Solubilization in Aqueous Media; American Chemical Society-Oxford: New York, 1998; Chapter 7.

14. Dowling, K. C.; Thomas, J. K. Macromolecules 1990, 23, 1059.

15. (a) Choi, S.; Briber, R. M.; Bauer, B. J.; Topp, A. Gauthier, M.; Tichagwa, L. Macromolecules 1999, 32, 7879. (b) Choi, S.; Briber, M. R.; Bauer, B. J.; Liu, D.-W.; Gauthier, M. Macromolecules 2000, 33, 6495.

16. Liu, G.; Zhou, J. Macromolecules 2003, 36, 5279.

17. Mackay, D; Shiu, W. Y.; Ma, K. C. Illustrated Handbook of Physical-Chemical Properties and Environmental Fate for Organic Chemicals; Lewis: Boca Raton, 1992.

18. Wilhelm, M.; Zhao, C.-L.; Wang, Y.; Xu, R.; Winnik, M. A.; Mura, J.-L.; Riess, G.; Croucher, M. D. Macromolecules 1991, 24, 1033.

19. Zhao, J.; Allen, C.; Eisenberg, A. Macromolecules 1997, 30, 7143.

20. Almgren, M.; Grieser, F.; Thomas, J. K. J. Am. Chem. Soc. 1979, 101, 279.

21. (a) Gauthier, M.; Li, W.; Tichagwa, L. Polymer 1994, 38, 6363. (b) Sheiko, S.; Gauthier, M.; Möller, M. Macromolecules 1997, 30, 2343. (c) Hempenius, M. A.; Zoetelief, W. F.; Gauthier, M.; Möller, M. Macromolecules 1998, 31, 2299.

22. Ismail, A. A.; Gouda, M. W.; Motawi, M. M. J. Pharm. Sci. 1970, 59, 220. 
23. Alkahmis, K. A.; Allaboun, H.; Al-Momami, W. Y. J. Pharm. Sci. 2003, 92, 839.

24. Hrdlovič, P.; Lukáč I. J. Photochem. Photobiol. A: Chem. 2000, 133, 73.

25. Nah, J.-W.; Jeong, Y.-II.; Cho, C.-S. Bull. Korean Chem. Soc. 2000, 21, 383.

26. Kalyuzhny, G.; Schneeweiss, M. A.; Shanzer, A.; Vaskevich, A.; Rubinstein, I. J. Am. Chem. Soc. 2001, 123, 3177.

27. (a) Kalyanasundaram, K.; Thomas, J. K. J. Am. Chem. Soc. 1977, 99, 2039. (b) Karpovich, D. S.; Blanchard, G. J. J. Phys. Chem. 1995, 99, 3951.

28. (a) Tedeschi, C.; Möhwald, H.; Kirstein, S. J. Am. Chem. Soc. 2001, 123, 954. (b) Chu, D.-Y.; Thomas, J. K. Macromolecules 1987, 20, 2133.

29. Claracq, J.; Santos, S. F. C. R.; Duhamel, J.; Dumousseaux, C.; Corpart, J.-M. Langmuir 2002, 18, 3829.

30. Anghel, D. F.; Toca-Herrera, J. L.; Winnik, F M.; Rettig, W.; Klitzing, R. V. Langmuir 2002, 18,5600 .

31. (a) Winnik, F. M. Chem. Rev. 1993, 93, 587. (b) Siu, H.; Duhamel, J. Macromolecules $\mathbf{2 0 0 5}, 38,7184$.

32. (32) (a) Todesco, R. V.; Basheer, R. A.; Kamat, P. V. Macromolecules 1986, 19, 2390. (b) Winnik, F. M.; Winnik, M. A.; Tazuke, S.; Ober, C. K. Macromolecules 1987, 20 , 38. 


\section{Chapter 6}

Sustained Release Properties of Arborescent Polystyrenegraft-Poly(2-vinylpyridine) Copolymers 


\subsection{Abstract}

The release kinetics of small molecules from dendritic copolymer micelles incorporating an arborescent polystyrene (PS) core and a poly(2-vinylpyridine) (P2VP) shell were investigated in dilute $\mathrm{HCl}$ solutions by fluorescence and UV spectroscopy. The redistribution of pyrene and perylene among arborescent micelles was studied by the fluorescence resonance energy transfer (FRET) technique, and was characterized by an initial burst in exchange followed by gradual equilibration of the probes. Fluorescence quenching experiments demonstrated that the diffusion coefficient of pyrene increased for copolymer micelles of higher generations, suggesting a more porous shell structure for the higher generation arborescent PS-g-P2VP copolymers. In vitro release tests for indomethacin and lidocaine monitored by UV spectroscopy showed that sustained release characteristics were achieved, the release rate being higher for lidocaine due to its higher water solubility at low $\mathrm{pH}$. The release rate of indomethacin increased for lower generation micelles and for higher micelle loadings, in agreement with a diffusion-controlled release mechanism. An increasing fraction of the indomethacin molecules loaded in the micelles also remained trapped for higher generation copolymers. The diffusion coefficient and the release rate of indomethacin were calculated by fitting the solution of Fick's second law of diffusion to the experimental data. While the initial release rate decreased for higher generations, the trends observed for the diffusion coefficients were similar to those determined for pyrene in the fluorescence quenching experiments. This result is again consistent with a more diffuse shell structure for higher generation micelles, possibly due to increased electrostatic repulsions between the charged P2VP chains. 


\subsection{Introduction}

Controlled release formulations have become important in our daily lives. These formulations offer great advantages over conventional delivery systems ${ }^{1}$ and are used in numerous applications including drug delivery systems, ${ }^{2}$ fertilizers, ${ }^{3}$ herbicides, ${ }^{4}$ and pesticides. ${ }^{5}$ Polymeric micelles have attracted much attention as controlled release devices, specially for hydrophobic compounds. ${ }^{6}$ Biocompatible amphiphilic block copolymers, for example, have been investigated for the controlled release of hydrophobic drugs. ${ }^{6 e}$ These micelles are able to solubilize large quantities of active molecules in their hydrophobic core and to gradually release them in a controllable manner. The use of unimolecular micelles as controlled release vehicles is particularly interesting ${ }^{7}$ because of their very stable, covalently bonded structure. For example, indomethacin molecules were encapsulated in unimolecular micelles based on a hydrophobic dendrimer core and a hydrophilic shell. ${ }^{8}$ In contrast to the rapid equilibration time $(4 \mathrm{~h})$ observed when free indomethacin dissolved in phosphate buffer solution was dialyzed against phosphate buffer solution at $37{ }^{\circ} \mathrm{C}$, equilibration was observed only after about $25 \mathrm{~h}$ for the encapsulated molecules.

Arborescent polystyrene-graft-poly(2-vinylpyridine) (PS-g-P2VP) copolymers are dendritic unimolecular micelles composed of a glassy branched PS core grafted with hydrophilic P2VP chains forming a shell. The PS core is synthesized from polymeric chains assembled according to a generation-based scheme consisting of functionalization and grafting reaction cycles. Grafting PS chains $\left(M_{\mathrm{w}} \approx 5000\right)$ onto a randomly functionalized linear PS backbone $\left(M_{\mathrm{w}} \approx 5000\right)$ yields a comb-branched or generation $G 0$ PS core. Subsequent functionalization and grafting reactions lead to higher generation $(G 1, G 2 \ldots)$ PS cores. Coupling living P2VP chains $\left(M_{\mathrm{w}} \approx 5000\right)$ with PS cores randomly functionalized with 
grafting sites yields amphiphilic macromolecules expected to encapsulate and slowly release hydrophobic molecules in a sustained fashion, in analogy to block copolymer micelles. Additional advantages of the arborescent micelles relatively to block copolymer micelles include enhanced structural stability through covalent bonding, and potentially the ability to control the release process through variations in the structure (e.g. the branching density) of the micelles.

It is clear that the polystyrene and poly(2-vinylpyridine) components of arborescent PS- $g$-P2VP copolymers are not biocompatible and that the testing conditions $(0.05 \mathrm{M} \mathrm{HCl}$ solutions), necessary to solubilize the copolymers in water, are far from those typically used for controlled delivery systems. The main goal of this study was to investigate the release of small molecules from arborescent copolymers as model highly branched micelles, to improve the understanding of the influence of branching on the release process. Fluorescence techniques were first applied to obtain information on the release of pyrene, a highly hydrophobic probe molecule, from arborescent PS-g-P2VP copolymers of generations $G 1$, G2, and G3 (Figure 6.1). In the second part of the investigation, the in vitro release of indomethacin was monitored by UV spectroscopy. The release kinetics were analyzed using the power law model and Fick's second law of diffusion. Release experiments for lidocaine from a $G 1$ copolymer were also carried out to study the influence of probe structure on the release process. 


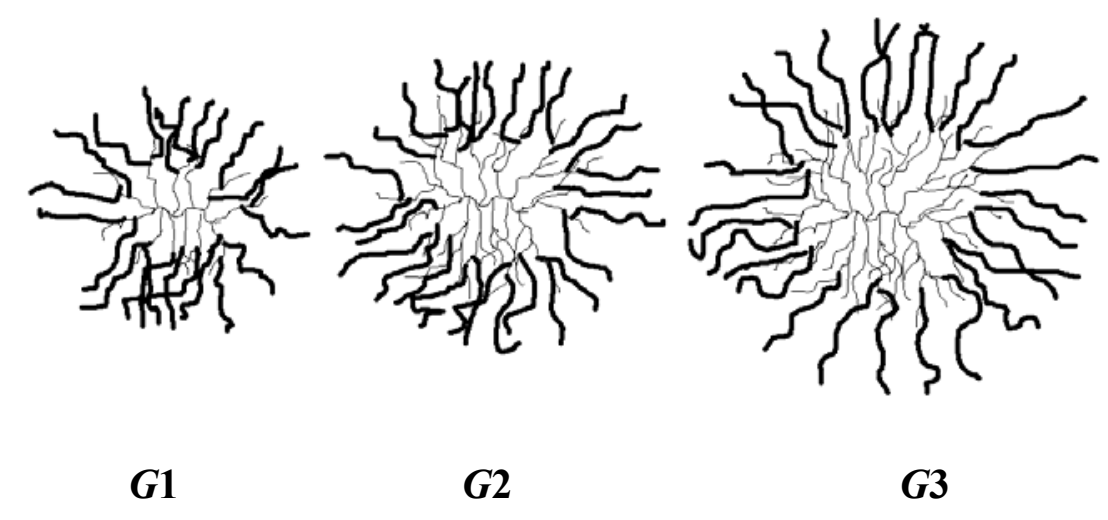

Figure 6.1. Three generations of arborescent PS- $g$-P2VP copolymers.

\subsection{Experimental Section}

6.3.1 Materials. Pyrene (99.9\%), perylene (sublimed, $99.5+\%$ ), and thallium (I) nitrate $(99.9 \%)$ were purchased from Aldrich. Indomethacin (99\%) and lidocaine were obtained from Sigma, while chloroform (HPLC grade) and methylene chloride (HPLC grade) were supplied by Merck. All chemicals were used as received. Milli-Q water (18 M $\Omega . c m)$ was used to prepare all sample solutions. The synthesis of the arborescent PS-g-P2VP copolymers used for this work was achieved previously ${ }^{9}$ by grafting 'living' P2VP chains onto acetylated PS substrates of generations $G 0-G 2$. All copolymers had narrow molecular weight distributions $\left(M_{\mathrm{w}} / M_{\mathrm{n}}<1.1\right)$ and PS contents of at most $18 \%$ by weight.

6.3.2 Sample Preparation. Micellar solutions were prepared by dissolving $250 \mathrm{mg}$ of arborescent PS- $g$-P2VP copolymer in $50 \mathrm{~mL}$ of $0.05 \mathrm{M}$ aqueous $\mathrm{HCl}$. The micelles were loaded either with pyrene or perylene, by adding approximately $10 \mathrm{mg}$ of the dyes to $5 \mathrm{~mL}$ of the copolymer solutions. The mixtures were stirred for three days and filtered through 0.22 $\mu \mathrm{m}$ mixed-ester membrane filters to remove solid dye residues. Loading of lidocaine and indomethacin in the copolymer was achieved by dissolving $50 \mathrm{mg}$ of a drug and $50 \mathrm{mg}$ of the 
copolymer in $1 \mathrm{~mL}$ of chloroform (lidocaine) or methylene chloride (indomethacin). The copolymer solutions were then precipitated in hexane. Non-encapsulated drug molecules were removed by washing the precipitates several times in hexane until no traces of the drugs were detected in the solvent with UV measurements.

6.3.3 Fluorescence Studies. Steady-state fluorescence spectra for the pyrene/perylene FRET and pyrene quenching experiments were acquired on a Photon Technology International LS-100 spectrometer using $1.0 \times 1.0 \mathrm{~cm}^{2}$ Hellma quartz cells. The slit widths on the excitation and emission monochromators were set at 2 and $1 \mathrm{~nm}$, respectively. For both the energy transfer and the quenching measurements, the excitation wavelength for the emission spectra was set at $344 \mathrm{~nm}$ and before each measurement, a 1pyrenemethanol standard was run to minimize inconsistencies resulting from lamp intensity variations. Time-resolved fluorescence decay curves were obtained by the time-correlated single photon counting technique using the IBH 5000U System from HORIBA Jobin Yvon Inc., with a nanosecond xenon flash lamp. The excitation wavelength used was $344 \mathrm{~nm}$ and the emission fluorescence decay was monitored at $372 \mathrm{~nm}$. A $371 \mathrm{~nm}$ cut-off filter was used to prevent scattered light interference in the detector. To obtain quantitative information from the decays, the curves were fitted with a sum of exponentials using the Marquard-Levenberg nonlinear least squares method. ${ }^{10}$ To obtain good quality fits as estimated from the $\chi^{2}$ values, the distribution and auto-correlation functions of the residuals, a sum of 4 exponentials was used. To minimize inner filter effects, the solutions for the fluorescence studies were diluted to obtain optical density values below 0.3 . The FRET measurements were started immediately after mixing $1.5 \mathrm{~mL}$ each of the micellar solutions loaded with pyrene and perylene. The emission intensity of the acceptor (perylene) was monitored at $475 \mathrm{~nm}$ to 
minimize any contributions from the emission of the donor (pyrene). For the quenching experiments, $20-40 \mu \mathrm{L}$ of a $0.2 \mathrm{M} \mathrm{TINO}_{3}$ solution were added to $3 \mathrm{~mL}$ of micellar solution loaded with pyrene. The fluorescence measurements were started after $10 \mathrm{~min}$ of equilibration.

6.3.4 Loading and Release of Indomethacin and Lidocaine. A known amount of drug-loaded arborescent PS-g-P2VP micelles (10 mg of lidocaine- or $2 \mathrm{mg}$ of indomethacinloaded micelles) was dissolved in $10 \mathrm{~mL}$ of $0.05 \mathrm{M} \mathrm{HCl}$. The amounts of drugs used in the experiments were adjusted so that the final concentrations of lidocaine and indomethacin in solution were similar. The micellar solutions were then transferred to a SPECTRA/POR ${ }^{\circledR} 7$ dialysis bag $(\mathrm{MWCO}=1000)$ and dialyzed against $100 \mathrm{~mL}$ of $0.05 \mathrm{M} \mathrm{HCl}$. At predetermined time intervals $3 \mathrm{~mL}$ aliquots of the dialysate were removed for UV measurements. To maintain suitable sink conditions and a constant dialysate volume, $3 \mathrm{~mL}$ of fresh $0.05 \mathrm{M} \mathrm{HCl}$ were added to the dialysate after each withdrawal. The UV measurements were performed on a Hewlett-Packard 8452A Diode Array Spectrophotometer, after dilution of the samples to obtain absorbance readings $<1.5$.

\subsection{Results and Discussion}

The characteristics of the arborescent PS- $g$-P2VP copolymers used in the experiments are reported in Table 6.1. The copolymers are based on PS cores of different generations, but all have P2VP segments of similar molecular weight $\left(M_{\mathrm{w}} \approx 5000\right)$. The lower loadings attained for perylene as compared to pyrene was explained in the previous chapter by its larger size. 
Table 6.1. Characteristics of PS-g-P2VP Arborescent Copolymers Used in the Study.

\begin{tabular}{lccc}
\hline Overall generation & $\boldsymbol{G 1}$ & $\boldsymbol{G 2}$ & $\boldsymbol{G 3}$ \\
\hline $\boldsymbol{M}_{\mathbf{w}}(\mathbf{g} / \mathbf{m o l})$ & $4.7 \times 10^{5}$ & $3.7 \times 10^{6}$ & $2.2 . \times 10^{7}$ \\
PS weight fraction (\%) & 12 & 13 & 18 \\
$\boldsymbol{f}_{\mathrm{w}}(\mathbf{c o r e})$ & 11 & 84 & 690 \\
$\boldsymbol{f}_{\mathrm{w}}(\mathbf{c o p o l y m e r})$ & 82 & 630 & 3400 \\
Pyrene loading (mol/g micelles) & $1.32 \times 10^{-5}$ & $1.0 \times 10^{-5}$ & $2.4 \times 10^{-5}$ \\
Perylene loading (mol/g micelles) & $1.1 \times 10^{-6}$ & $4.2 \times 10^{-7}$ & $9.6 \times 10^{-7}$ \\
\hline $\begin{array}{l}\text { fis the branching functionality, defined as the number of side chains added in the } \\
\text { last grafting cycle. }\end{array}$ &
\end{tabular}

6.4.1 Nonradiative Energy Transfer. Fluorescence resonance energy transfer (FRET) is based on a dipole-dipole coupling process allowing the transfer of energy from an excited fluorescence donor molecule to a ground state acceptor molecule without emission of a photon. ${ }^{11}$ The rate constant for the FRET process, $k_{T}$, is dependent among others on the donor-to-acceptor distance $r$, according to the equation

$$
k_{T}=\frac{1}{\tau_{D}}\left(\frac{R_{0}}{r}\right)^{6}
$$

where $\tau_{D}$ is the decay constant for the donor in the absence of the acceptor, and $R_{0}$ is the Förster radius, a parameter depending on the photophysical properties of the donor-acceptor pair including the extent of overlap of the donor emission and the acceptor absorbance spectra. $^{12}$ It also represents the distance at which the energy transfer efficiency is $50 \%$. Timedependent FRET measurements upon mixing two separate solutions of identical micellar concentrations, one loaded with donor molecules and the other one with acceptor molecules, 
have been used to characterize the release kinetics of hydrophobic probes from block copolymer micelles. ${ }^{13}$ In the current study, the donor molecule selected is pyrene and the acceptor is perylene. The emission spectrum of pyrene partly overlaps with the absorption spectrum of perylene, as shown in Figure 6.2 with the corresponding emission spectrum for perylene, and the pair has an $R_{0}$ value of $33.32 \mathrm{~nm} .{ }^{14}$ Mixing of the loaded micelles initiates the redistribution of the fluorophores among the micelles, and the time-dependent increase in the steady state perylene emission is monitored at $475 \mathrm{~nm}$ upon excitation of pyrene. Since the efficiency of FRET depends on diffusion, it is possible to use this method to investigate the influence of arborescent PS-g-P2VP branching functionality on the probe release kinetics, as the branching functionality increases with the generation number.

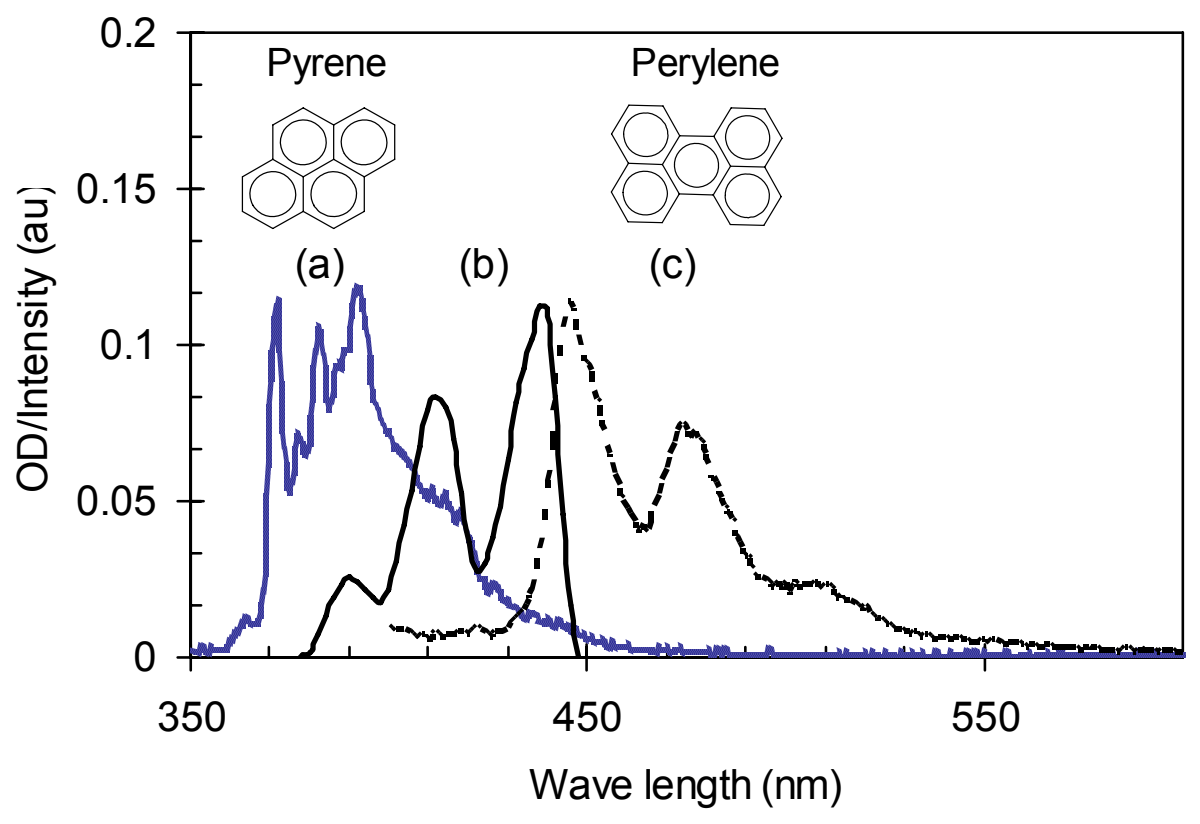

Figure 6.2. Emission spectrum for pyrene excited at $344 \mathrm{~nm}$ (a), overlap with absorption spectrum for perylene (b), resulting emission spectrum for perylene (c). All spectra were recorded in toluene, and normalized to be on the same scale. 
The normalized experimental time-dependent fluorescence intensity ratio for perylene, $R_{\mathrm{A}}$, used to express the FRET results shown in Figure 6.3 , is defined by the equation

$$
R_{A}=\frac{I_{A}(t)-I_{A}(0)}{I_{A}(\infty)-I_{A}(0)}
$$

where $I_{\mathrm{A}}(t)$ and $I_{\mathrm{A}}(0)$ are the perylene emission intensities at time $t$ and at the beginning of the measurements, respectively, and $I_{\mathrm{A}}(\infty)$ is the intensity at equilibrium. A rapid initial increase in $R_{\mathrm{A}}$ is observed during the first $300 \mathrm{~min}$, and is followed by more gradual equilibration at longer times (Figure 6.3). This corresponds to an initial burst in release followed by slow equilibration. While the overall release profile is very similar for all copolymers, there is a small influence of copolymer structure on the initial burst release.

It is seen (Figure 6.3 inset) that the initial release rate is higher for the $G 1$ copolymer than for the $G 2$ and $G 3$ molecules. This is attributed to the lower branching functionality of the $G 1$ sample (Table 6.1). As discussed in the Chapter 5, pyrene and perylene are highly hydrophobic and should therefore be located in the PS core of the micelle. The diffusion of the probes in the core should be influenced by their branching functionality, the copolymer with a higher core branching functionality $(G 3)$ presumably having a lower initial release rate than the $G 2$ and the $G 1$ copolymers. However, the initial release rate of the $G 2$ and $G 3$ copolymers is essentially identical. Because of its higher PS content, sample $G 3$ has a higher probe loading than sample $G 2$ (Table 6.1). The influence of branching functionality on the initial release rate for this sample may be counterbalanced by its higher initial probe concentration, as the flux of solute should increase with the initial concentration of the probes in the micelles. 


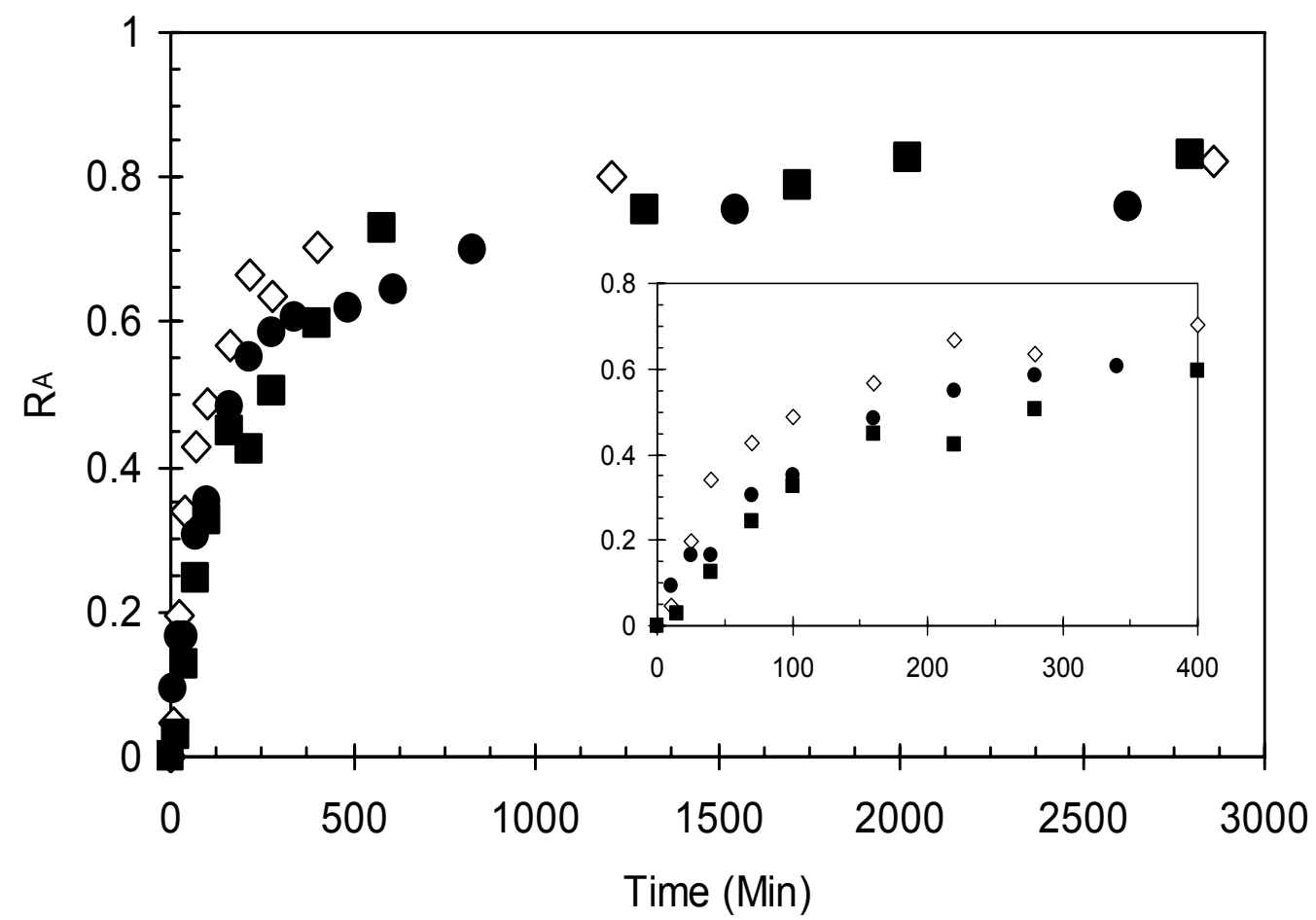

Figure 6.3. Normalized perylene intensity ratios $R_{\mathrm{A}}$ for $G 1(\diamond) G 2(\bullet)$ and $G 3(\bullet)$ arborescent copolymers. The initial portion of the plot is expanded in the inset.

6.4.2 Fluorescence Quenching Experiments. To clarify the influence of branching functionality on the initial release process, it is important to understand how easily small molecules can penetrate in the micelles. With fluorescence collision quenching experiments, it is possible not only to quantify the diffusion rate for small molecules but also to obtain information on the distribution of the probe molecules within the micelles. ${ }^{15}$

In a series of quenching experiments, $20-40 \mu \mathrm{L}$ aliquots of $0.2 \mathrm{M} \mathrm{TINO}_{3}$ solutions were added to a 3-mL sample of micellar solution loaded with pyrene. As an ionic quencher, thallium nitrate is expected to quench only pyrene molecules located within the P2VP shell or at the PS core-P2VP interface, since $\mathrm{Tl}^{+}$ions cannot penetrate inside the hydrophobic PS 
core. ${ }^{13 a, 16}$ It is therefore possible to quantify the fraction of pyrene molecules accessible to the quencher and the fraction buried within the PS core.

The kinetics of collisional fluorescence quenching in homogeneous solutions is best described by the Stern-Volmer equation,

$$
\frac{I_{0}}{I}=1+k_{q} \tau_{0}[Q]
$$

where $I_{0}$ and $\tau_{0}$ represent the unquenched fluorescence intensity and the lifetime of the fluorophore, respectively, $I$ is the fluorescence intensity observed at a quencher concentration $[Q]$, and $k_{\mathrm{q}}$ is the bimolecular quenching constant. The intensity of pyrene emission was determined by integration of the spectrum from $370-374 \mathrm{~nm}$, and the numberaverage fluorescence lifetime $\langle\tau\rangle_{0}$, determined from tetraexponential fitting of the decay curves, was substituted for $\tau_{0}$ in Equation 6.3. A plot of $I_{0} / I$ against $[Q]$ is expected to be linear, but a plateau may be present at high quencher concentrations if protective quenching is present. ${ }^{15,17}$ The curves in Figure 6.4a clearly indicate that only a fraction of the pyrene molecules in the micelles was accessible to the ionic quencher, as the $I_{0} / I$ ratio reaches a plateau at high quencher concentrations for all pyrene-loaded arborescent copolymers. Under these conditions, the data can be analyzed using the modified Stern-Volmer equation

$$
\frac{I_{0}}{I_{0}-I}=\frac{1}{f_{a}}+\frac{1}{f_{a} k_{q} \tau_{0}[Q]}
$$

to determine the fraction of pyrene molecules, $f_{a}$, accessible to thallium nitrate.

To proceed with the analysis using the Stern-Volmer equations it was necessary to determine $\langle\tau\rangle_{0}$ from the time-resolved decay curves. An example of a decay profile 
obtained for a pyrene-loaded $G 1$ copolymer in the absence of quencher is shown on Figure 6.5 .
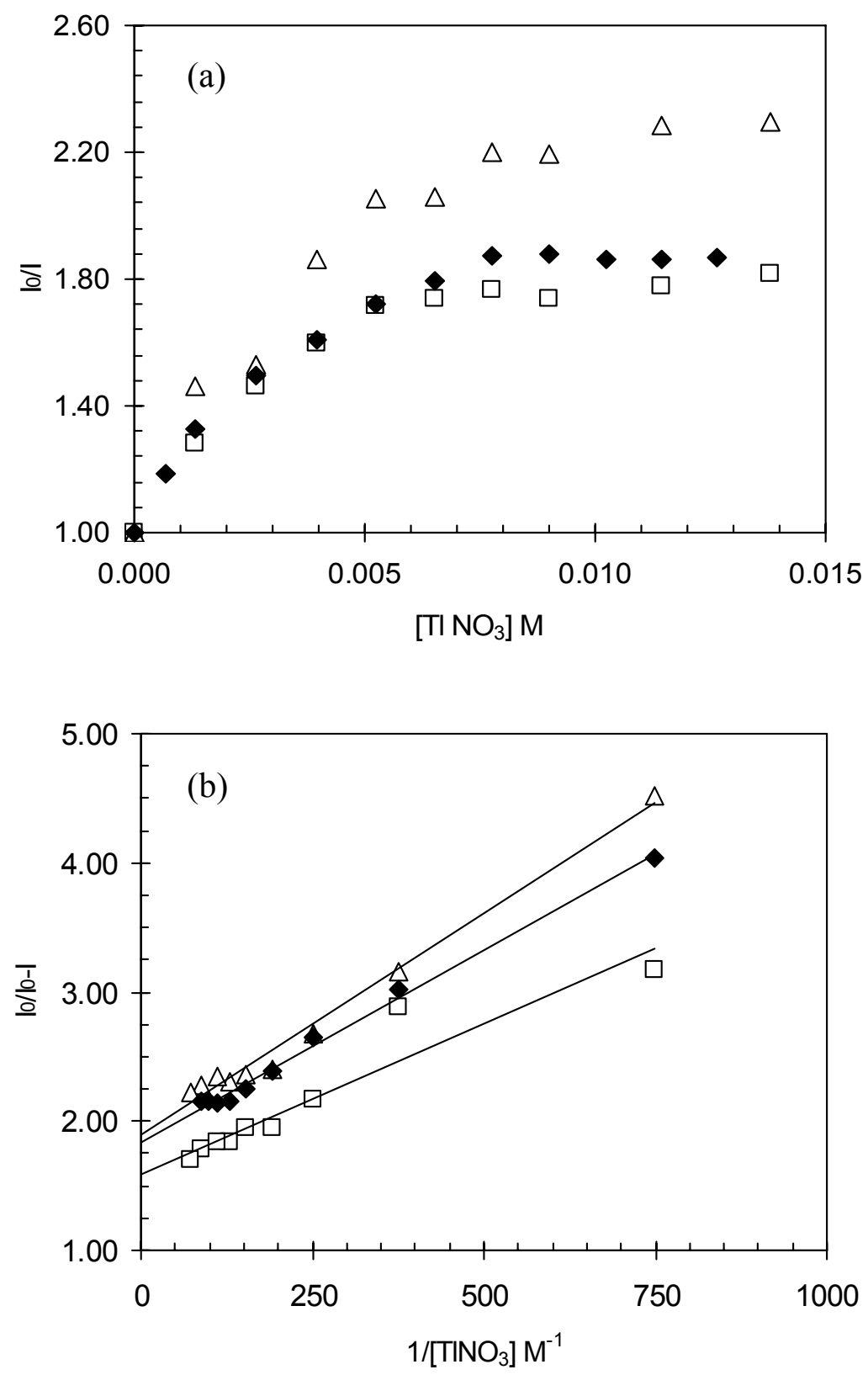

Figure 6.4. (a) Stern-Volmer and (b) modified Stern-Volmer plots for pyrene-loaded G1 ( $\square$ ), $G 2(\Delta)$, and $G 3(\downarrow)$ PS- $g$-P2VP copolymers. 

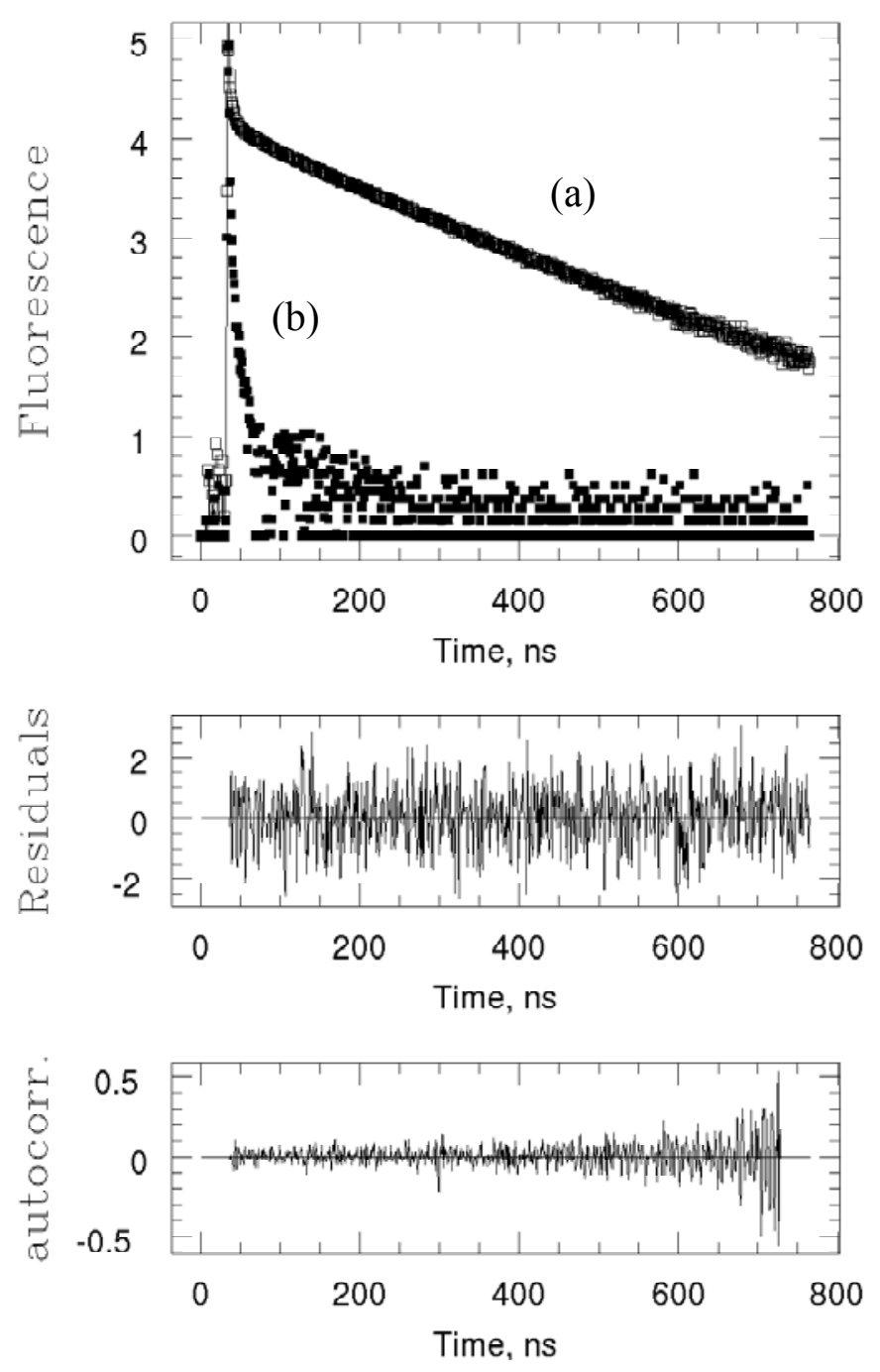

Figure 6.5. Decay curve for pyrene in $G 1$ arborescent PS-g-P2VP copolymer (a), and instrument response function at $\tau=0(b)$.

All the samples exhibited multiexponential decay behavior, indicating that the pyrene molecules were located in different environments of the micelles. In all cases, the best fit (lowest $\chi^{2}$ value) was obtained with four exponentials. The lifetimes and preexponential factors obtained from the fits are listed in Table 6.2. Theoretically, thorough analysis of the decay profiles could provide quantitative information on each pyrene population probing a 
given micellar environment. ${ }^{17}$ However, considering the complex nature of the decays possibly resulting from inherent quenching by preassociated pyrene molecules ${ }^{18}$ and by the protonated P2VP shell, ${ }^{19}$ the analysis was limited to the calculation of the number-average lifetimes in the present case.

Table 6.2. Lifetimes (ns) and Preexponential Factors Obtained from Fluorescence Decays.

\begin{tabular}{cccc}
\hline & $\boldsymbol{G 1}$ & $\boldsymbol{G 2}$ & $\boldsymbol{G 3}$ \\
\hline$\tau_{\mathbf{1}}\left(\boldsymbol{A}_{\mathbf{1}}\right)$ & $4.94(0.41)$ & $3.54(0.64)$ & $4.59(0.59)$ \\
$\boldsymbol{\tau}_{\mathbf{2}}\left(\boldsymbol{A}_{\mathbf{2}}\right)$ & $23.99(0.30)$ & $27.87(0.08)$ & $28.16(0.14)$ \\
$\boldsymbol{\tau}_{3}\left(\boldsymbol{A}_{3}\right)$ & $83.42(0.22)$ & $116.64(0.24)$ & $103.97(0.20)$ \\
$\boldsymbol{\tau}_{4}\left(\boldsymbol{A}_{4}\right)$ & $224.59(0.07)$ & $184.81(0.04)$ & $193.81(0.07)$ \\
$<\boldsymbol{\tau}>_{0}$ & 43.59 & 40.12 & 40.95 \\
$\chi^{2}$ & 1.128 & 1.024 & 1.136 \\
\hline
\end{tabular}

The data from Figure $6.4 \mathrm{~b}$ were combined with the $\langle\tau\rangle_{0}$ values in Table 6.2 to estimate the fraction $f_{\mathrm{a}}$ of pyrene accessible to the quencher and the bimolecular quenching constant $k_{\mathrm{q}}$. All the curves in Figure $6.4 \mathrm{~b}$ had correlation coefficients greater than 0.99 . The quenching constant $k_{\mathrm{q}}$ can be represented by the Smoluchowski equation

$$
k_{q}=4 \pi R D N_{A}
$$

where $D$ is the sum of the diffusion coefficients for the fluorophore and the quencher, and $R$ is the sum of the molecular radii of the fluorophore and the quencher. The value $R=8.6 \AA$ was calculated from the van der Waals radii of pyrene and thallium nitrate, using the molecular modeling software Hyperchem 7.5. 
The results obtained from the analysis of the quenching experiments are summarized in Table 6.3. The very low average fluorescence lifetime values obtained are attributed to the high fraction of species quenched by protonated P2VP chains or by other pyrene molecules. In a previous quenching study involving pyrene-labeled arborescent PS homopolymer solutions in THF, the bimolecular quenching constant was found to decrease for higher generations when nitromethane was used as quencher. ${ }^{20}$ The quencher molecules must diffuse into the highly branched arborescent PS to encounter the pyrene molecules. Since the segmental density of arborescent polymers increases with the generation number, $k_{\mathrm{q}}$ would be expected to decrease for higher generations. In the present study, the pyrene molecules in the PS core are not polymer-bound and $\mathrm{TINO}_{3}$ is an aqueous quencher. Encounters between the quencher and probe molecules are therefore likely limited to the interfacial and the shell regions of the micelles. Consequently, the bimolecular collision constant (and hence the diffusion coefficient) should depend on the porosity of these regions. Segmental electrostatic repulsions are known to influence the structure of polyelectrolytes in solution. ${ }^{21,22}$ For example, the hydrodynamic diameter of PS- $b$-P2VP- $b$-PEO triblock copolymer micelles in aqueous solutions was found to increase from 75 to $135 \mathrm{~nm}$ as the $\mathrm{pH}$ dropped below $5 .^{22}$ The behavior of arborescent PS-g-P2VP copolymers should be likewise influenced by the polyelectolyte character of the protonated P2VP segments in the shell.

The bimolecular quenching constant actually increases from samples $G 0$ to $G 2$, leading to an increase in diffusion coefficient $D$, as it is directly related to $k_{\mathrm{q}}$ through Equation 6.5. This trend is opposite to the results obtained for nitrobenzene quenching of (neutral) pyrene-labeled arborescent polystyrenes. ${ }^{20}$ The increase in $k_{\mathrm{q}}$ and $D$ observed from 
the generation $G 0$ to $G 2$ copolymers is attributed to the large number of protonated P2VP segments increasing the electrostatic repulsions in the copolymer molecules. The increase in

Table 6.3. Quenching Data for Pyrene-Loaded Arborescent PS-g-P2VP.

\begin{tabular}{ccccc}
\hline & $\boldsymbol{f}_{\mathbf{a}}(\mathbf{\%})$ & $<\tau>_{\mathbf{0}} / \mathbf{n s}$ & $\boldsymbol{k}_{\mathbf{q}} / \mathbf{1 0}^{\mathbf{1 0}} \mathbf{M}^{-1} \mathbf{s}^{-1}$ & $\boldsymbol{D} / \mathbf{1 0} \mathbf{0}^{-\mathbf{5}} \mathbf{c m}^{2} \mathbf{s}^{-1}$ \\
\hline $\boldsymbol{G} \mathbf{0}^{\mathbf{a}}$ & $45 \pm 1$ & 53.83 & $0.58 \pm 0.02$ & $0.89 \pm 0.03$ \\
$\boldsymbol{G 1}$ & $53 \pm 2$ & 43.59 & $1.27 \pm 0.03$ & $1.94 \pm 0.01$ \\
$\boldsymbol{G 2}$ & $63 \pm 2$ & 40.12 & $2.93 \pm 0.02$ & $4.48 \pm 0.02$ \\
$\boldsymbol{G 3}$ & $55 \pm 1$ & 40.95 & $1.48 \pm 0.01$ & $2.26 \pm 0.02$ \\
\hline
\end{tabular}

${ }^{a}$ While the $G 0$ arborescent PS- $g$-P2VP copolymers have been found to aggregate in $0.1 \mathrm{M}$ $\mathrm{HCl}$ aqueous solutions, ${ }^{9}$ it is assumed that the microporosity of the copolymer molecules is not affected by the formation of aggregates.

$D$ from $0.89 \times 10^{-8} \mathrm{~cm}^{2} . \mathrm{s}^{-1}$ for the $G 0$ or comb-branched copolymer $\left(f_{\mathrm{w}}=12\right)$ to $D=4.48 \times$ $10^{8} \mathrm{~cm}^{2} \cdot \mathrm{s}^{-1}$ for the $G 2$ copolymers $\left(f_{\mathrm{w}}=690\right)$ is consistent with increased electrostatic repulsions in the interfacial and P2VP shell regions facilitating the penetration of quencher molecules into the micelles. For the same reason, it is not surprising that the fraction of pyrene molecules accessible to thallium nitrate $\left(f_{\mathrm{a}}\right)$ increases from $G 0$ to $G 2$. The $k_{\mathrm{q}}$ and $D$ values determined for the $G 3$ copolymer sample are unexpectedly low. While the cause of this deviation is not very clear, it could be due to the dominating influence of the high branching functionality of the copolymer $\left(f_{\mathrm{w}}=3400\right)$ on its structural rigidity.

It would have been interesting to analyze the time-dependent energy transfer curves using known release kinetic models, however due to the uncertainty of the approach, and the complex nature of the FRET process renders such an analysis extremely difficult. ${ }^{13 b}$ The 
distribution of fluorophores among micelles is a multi-step process that comprises the release from one set of micelles, diffusion through the aqueous medium, and uptake by the other micelles. Furthermore, there is difficulty in relating fluorescence intensities to the amount of probe released because the pyrene fluorescence is quenched by preassociated species, and both probes can be quenched by protonated P2VP chains. ${ }^{23}$ To avoid these problems, indomethacin was selected to study the release kinetics from arborescent PS-g-P2VP copolymers by dialysis, for comparison with the results obtained in the fluorescence studies. Release studies for lidocaine were also performed with the $G 1$ copolymers, to study the influence of probe structure on the release process.

6.4.3 In vitro Release Kinetics of Indomethacin and Lidocaine. Indomethacin and lidocaine were selected as model drugs for the release experiments on the basis of their ready availability and of the large body of literature available on that topic. Total drug loading was determined by dissolving the loaded micelles in ethanol and measuring the UV absorption of the solution at $\lambda_{\max }=232 \mathrm{~nm}$ for indomethacin and $\lambda_{\max }=262 \mathrm{~nm}$ for lidocaine. Loadings of $46 \%, 42 \%$, and $43 \%$ indomethacin by weight were obtained for the $G 1, G 2$, and $G 3$ copolymers, respectively. For lidocaine, a loading of $8 \%$ by weight was obtained for the $G 1$ copolymer. The low encapsulation efficiency observed for lidocaine (Figure 6.6b) is presumably due to its high solubility in hexane and weak interactions with the micelles. In contrast, indomethacin (Figure 6.6a) is sparingly soluble in hexane and is expected to interact strongly with the P2VP segments of the micelles. The encapsulation efficiency has been found to increase with decrease in the solubility of the small molecules in the recovery medium. ${ }^{24}$ 


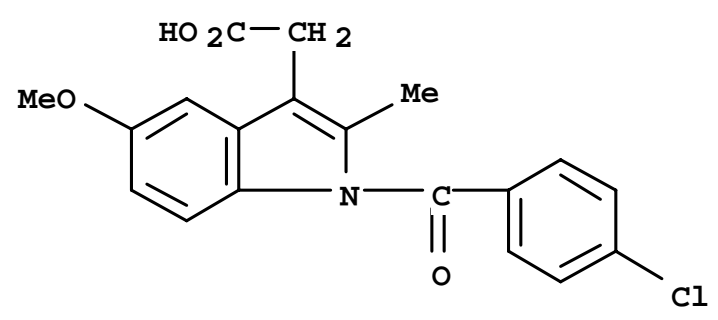

(a)

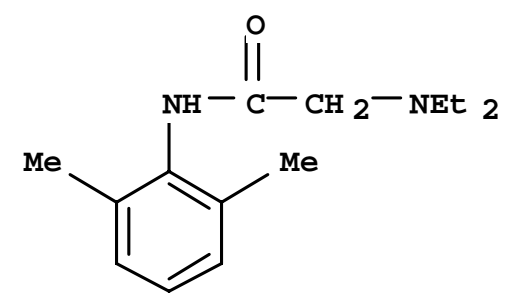

(b)

Figure 6.6. Structures of indomethacin (a) and lidocaine (b).

The release profiles obtained in $0.05 \mathrm{M} \mathrm{HCl}$ for indomethacin- and lidocaine-loaded G1 arborescent PS-g-P2VP copolymers are compared in Figure 6.7. In contrast to the rapid release observed for the free probes from dialysis tubing ( $100 \%$ release in less than $5 \mathrm{~h})$, the release from the dendritic micelles is slow, corresponding to sustained characteristics. Each release experiment was performed twice. $t$ test values obtained from the release data didn't show any statistical difference between the results at a confidence level of $95 \%$. For both indomethacin and lidocaine, an initial burst in release is observed for the first $5 \mathrm{~h}$ but is followed by more gradual release until equilibration is attained over $1-2$ days. Furthermore, while roughly $80 \%$ of the loaded lidocaine is released at equilibrium, only about $40 \%$ of the indomethacin is released from the micelles (Figure 6.8). Lidocaine is less hydrophobic (LogP $=2.4 \pm 0.3$ ), a large fraction of the probe is presumably located in the unprotonated P2VP shell after encapsulation. This fraction is completely released within the time frame of the release experiment.

The incomplete release of indomethacin from the micelles cannot be attributed simply to reservoir (dialysate) saturation, as a similar mass fraction versus time profile was obtained in a preliminary experiment with a doubled dialysate volume. The incomplete release is more likely related to physical entrapment of the drug in the hydrophobic core and/or the palisade 


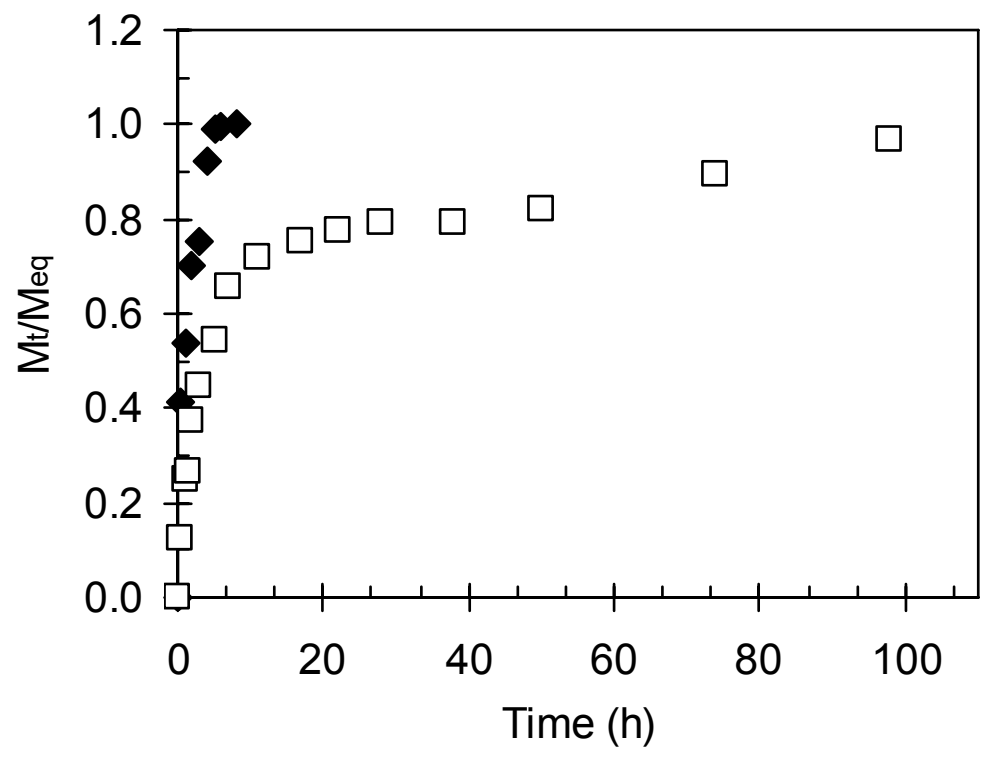

(a)

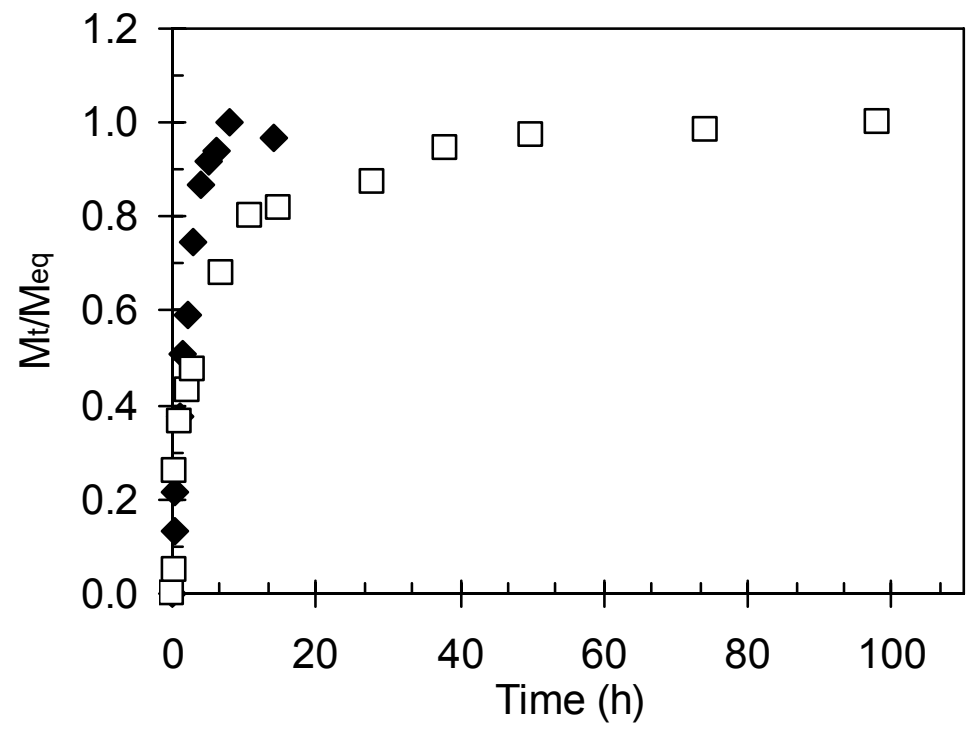

(b)

Figure 6.7. Release profile for (a) indomethacin and (b) lidocaine in $0.05 \mathrm{M} \mathrm{HCl}$ in the free state $(\diamond)$ and from $G 1$ PS- $g$-P2VP copolymer $(\square) . \mathrm{M}_{\mathrm{t}}$ is the cumulative weight released at time $\mathrm{t}$, and $\mathrm{M}_{\mathrm{eq}}$ is at equilibrium. 


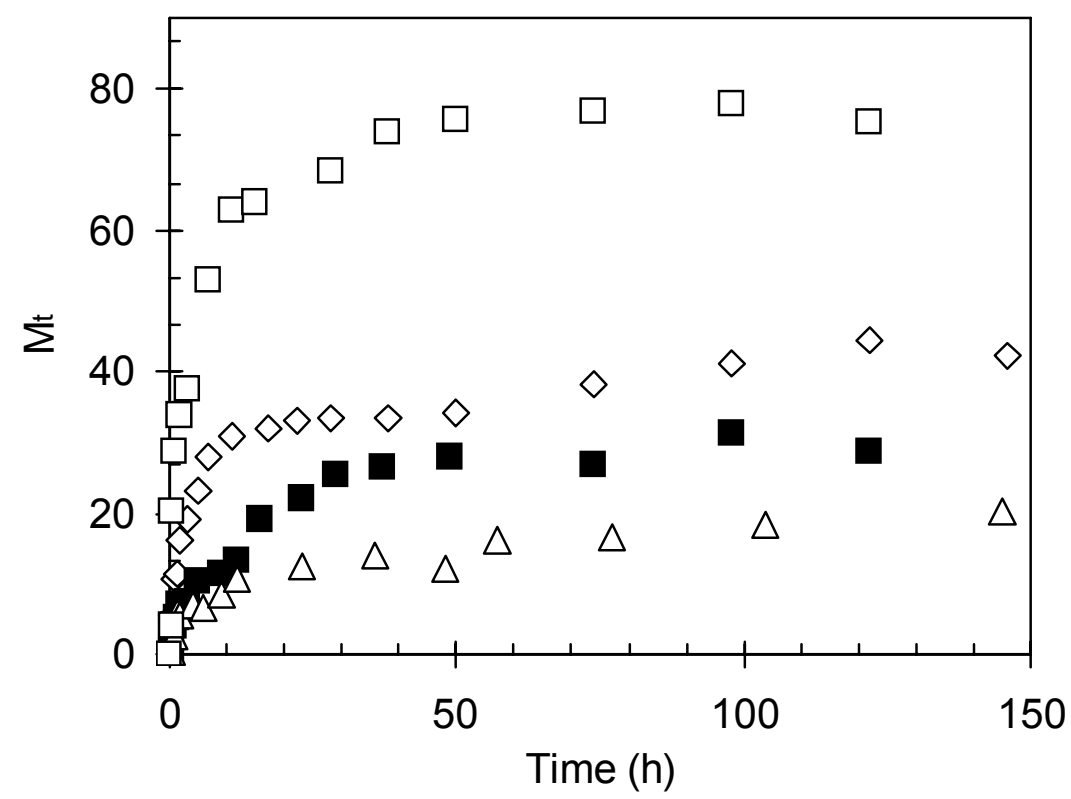

Figure 6.8. Percentage mass fraction of indomethacin released from $G 1(\diamond), G 2(\mathbf{\square})$, and $G 3$ $(\Delta)$ copolymers, and lidocaine released from the $G 1(\square)$ copolymer.

(interfacial) regions of the micelles, due to the moderately hydrophobic character $(\log \mathrm{P}=$ $3.1 \pm 0.4)$ of indomethacin. A similar explanation was suggested for the incomplete release of indomethacin from block copolymer micelles incorporating a hydrophobic poly(€caprolactone) core and a hydrophilic poly(ethylene glycol) shell. ${ }^{25}$ Another possibility could be hydrogen bonding interactions between the pyridine ring of P2VP chain segments and the carboxylate group of indomethacin. The formation of hydrogen bonds between indomethacin and poly(vinyl pyrrolidone) units has been reported, ${ }^{26}$ and determined to involve the carboxylic acid group of indomethacin and the carbonyl group of the pyrrole ring. The lack of hydrogen bond formation with the nitrogen atom in the pyrrole ring was attributed to steric hindrance. In the PS-g-P2VP micelles, the nitrogen in the pyridine ring is not sterically hindered and should be capable of forming hydrogen bonds, since indomethacin was loaded 
in the micelles before dissolution in the $\mathrm{HCl}$ solution. There are no strong interactions between lidocaine and the micelles, which would likewise explain why a larger fraction of the encapsulated drug was released.

The influence of the micelle generation number on the release of indomethacin (Figure 6.8) is similar to that observed in the FRET studies using the fluorescent probes. The release profile for the $G 1$ micelles shows an initial burst in release, which decreases in magnitude for higher generations, the $G 3$ copolymer having a more linear profile. While the $G 1, G 2$, and $G 3$ copolymers had comparable micellar loadings, the amount of probe released at equilibrium decreased for higher generations. At equilibrium more than $40 \%$ of the indomethacin loaded in the G1 micelles was released (Figure 6.8), while about 30\% and less than $20 \%$ were released from the $G 2$ and $G 3$ samples, respectively. This result suggests that the number of probe molecules encapsulated in the highly branched glassy PS core/palisade regions of the micelles increase with the size of the micelles, and therefore the fraction of drug molecules release within the time frame of the experiment decreases.

The influence of indomethacin loading (controlled by varying the weight fraction of copolymer and drug during encapsulation) on the release process for $G 1$ micelles is illustrated in Figure 6.9. The initial release rate and the mass fraction of indomethacin released at equilibrium both increase with loading. For loadings of 29,33 , and $46 \% \mathrm{w} / \mathrm{w}$, for instance, the fraction of indomethacin released from the $G 1$ arborescent PS- $g$-P2VP copolymers after $20 \mathrm{~h}$ was 18,30 , and $33 \%$, respectively. The dependence of the release of indomethacin on its concentration in the micelles is consistent with a diffusion-controlled release mechanism. As the concentration of indomethacin in the micelles is increased the 
fraction of molecules in the P2VP shell presumably increases, leading to faster release of the probe.

The release data were analyzed using the power law model, ${ }^{27}$ a semi-empirical equation in the form

$$
\frac{M_{t}}{M_{e q}}=k t^{n}
$$

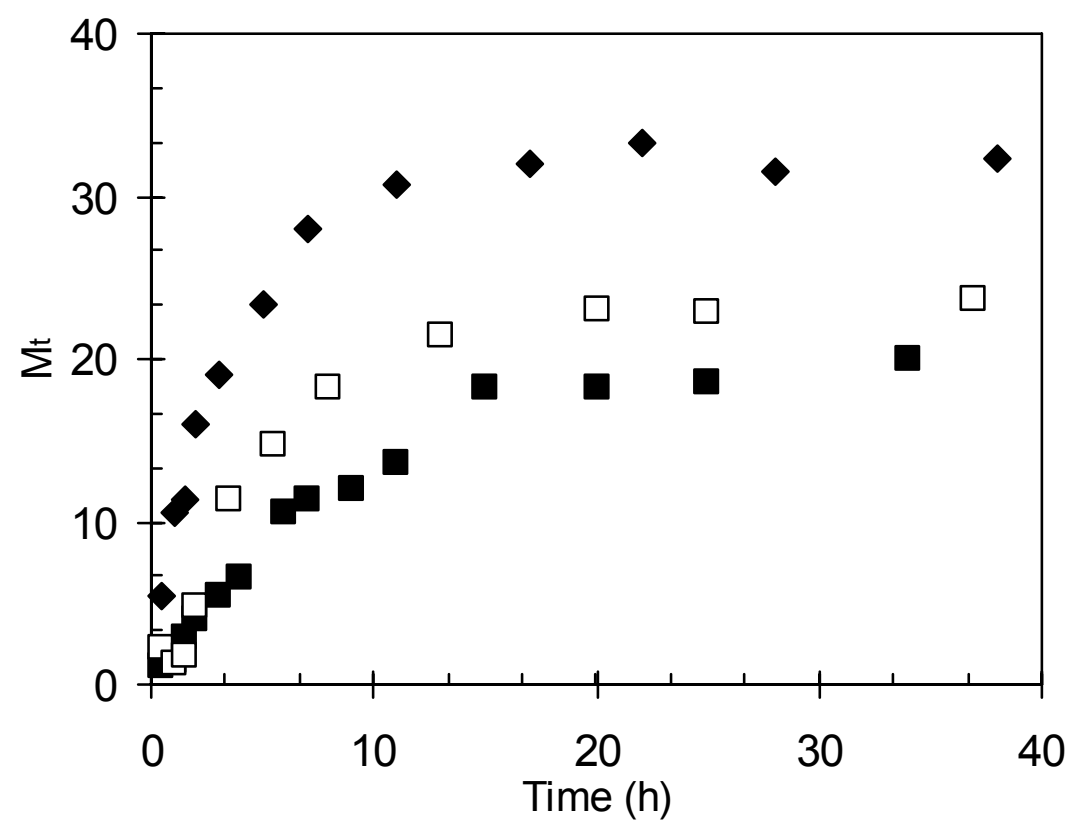

Figure 6.9. Influence of initial indomethacin loading (w/w) on release from $G 1$ micelles:

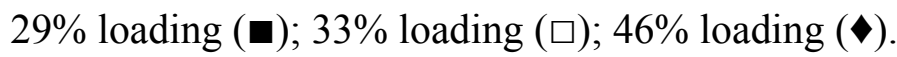

used to describe the release from polymeric systems for up to $55 \%$ of the equilibration level. ${ }^{28}$ In the power law model, $k$ is a constant dependent upon structural and geometric characteristics of the device. Its magnitude is usually indicative of solvent-solute or devicesolute interactions, high values reflecting strong solvent-solute or weak device-solute 
interactions. The release exponent $n$ depends on the release mechanism, $n=1.0$ representing a release rate independent of time or zeroth-order release kinetics. This type of release mechanism is also known as case-II transport and typically involves time-dependent swelling of the delivery device. A value of $n<0.43$ is indicative of Fickian diffusion control of the release process. Intermediate $n$ values $(0.43-1.0)$, corresponding to anomalous transport, may be regarded as a combination of both mechanisms. The values of $n$ quoted here are only valid for release devices with a spherical geometry, and actually vary with the shape of the device.

Experimental values of $k$ and $n$ were determined for the PS- $g$-P2VP micelles by the linear least squares method. It can be seen from Table 6.4 that for approximately $40 \%$ loading, the release mechanism for indomethacin changes from anomalous transport to Fickian (diffusion-controlled) as the generation number of the arborescent PS-g-P2VP copolymers increases. No clear trend can be observed among the $k$ values, although the $G 1$ copolymers had a significantly higher value suggesting the most favorable interactions between the probe and solvent. For comparison lidocaine, which is more soluble in $0.05 \mathrm{M}$ $\mathrm{HCl}$, had $n=0.34$ and $k=0.34$ in the $G 1$ micelles.

Table 6.4. Parameters $k$ and $n$ of the Power Law Model for the Release of Indomethacin from PS-g-P2VP Copolymers.

\begin{tabular}{cccc}
\hline & $\boldsymbol{G 1}$ & $\boldsymbol{G 2}$ & $\boldsymbol{G 3}$ \\
\hline $\boldsymbol{n}$ & $0.60 \pm 0.04$ & $0.48 \pm 0.01$ & $0.39 \pm 0.06$ \\
$\boldsymbol{k}$ & $0.21 \pm 0.03$ & $0.14 \pm 0.02$ & $0.15 \pm 0.04$ \\
\hline
\end{tabular}


Considering that the release of indomethacin from arborescent PS- $g$-P2VP copolymers is apparently dominated by diffusion, Fick's second law of diffusion was used to estimate the diffusion coefficient of indomethacin in the micelles and the initial burst release rate from the micelles to their immediate surroundings (i.e. $0.05 \mathrm{M} \mathrm{HCl}$ ). The amount of material diffusing out of a homogeneous sphere at time $t$ can be calculated from the exact solution to Fick's second law of diffusion given by ${ }^{29}$

$$
\frac{M_{t}}{M_{\infty}}=1-\frac{6}{\pi^{2}} \sum_{n=1}^{\infty} \frac{1}{n^{2}} \exp \left(-D n^{2} \pi^{2} t / r^{2}\right)
$$

where $D$ is the average diffusion coefficient of the substance within the sphere, $n$ is the number of iterations used for the calculations, and $r$ is the radius of the sphere. To calculate the initial burst release rate, the early time approximation of Equation $6.7^{30}$

$$
\frac{M_{t}}{M_{\infty}}=6\left(\frac{D_{i} t}{r^{2} \pi}\right)^{1 / 2}-\frac{3 D_{i} t}{r^{2}}
$$

can be derived with respect to time to give the early time release rate

$$
\frac{d\left(M_{t} / M_{\infty}\right)}{d t}=3\left(\frac{D_{i}}{r^{2} \pi t}\right)^{1 / 2}-\frac{3 D_{i}}{r^{2}} .
$$

Equations 6.8 and 6.9 are typically valid over the first $40 \%$ of the release process. The early time diffusion coefficient $\left(D_{\mathrm{i}}\right)$, the average diffusion coefficient $(D)$, and the early time release rate were determined by fitting Equations 6.7, 6.8, and 6.9, respectively, to the experimental data and by minimizing the $\chi^{2}$ value obtained using Mathcad 2000 Professional software.

The diffusion coefficients for indomethacin in arborescent PS-g-P2VP copolymers (Table 6.5) are much lower than those reported in the literature for the same probe in other 
controlled release devices. For example, $D \approx 3.5 \times 10^{-10} \mathrm{~cm}^{2} . \mathrm{s}^{-1}$ was reported for pressure sensitive-adhesive devices made from polystyrene-block-polyisoprene copolymers, ${ }^{2 a}$ and $D \approx$ $2.7 \times 10^{-16} \mathrm{~cm}^{2} \cdot \mathrm{s}^{-1}$ was reported for poly(L-lactic acid)-block-poly(ethylene glycol) micelles. ${ }^{6 \mathrm{~d}}$ The low $D$ values obtained herein (Table 6.5), on the order of $10^{-18} \mathrm{~cm}^{2} . \mathrm{s}^{-1}$, are attributed to the combined effects of the highly branched structure of the arborescent copolymers.

The trend among the diffusion coefficients $D$ for indomethacin in Table 6.5 is consistent with that determined for pyrene (Table 6.3). While a decrease in the diffusion coefficient might have been expected for higher generation copolymers due to their increased branching functionality, $D_{\mathrm{i}}$ generally increases. This is again attributed to increased electrostatic repulsions between $\mathrm{P} 2 \mathrm{VP}$ segments for higher generation copolymers upon protonation, as discussed earlier. After dissolution in dilute $\mathrm{HCl}$, protonation leads to electrostatic repulsions causing the P2VP chains to stretch, and to an increase in the volume of the hydrophilic shell. ${ }^{9,21,22}$ While the resulting volume expansion tends to be less significant for higher generation arborescent PS-g-P2VP copolymers, ${ }^{21}$ it appears that the electrostatic repulsions still suffice to increase the microporosity and the diffusion coefficients for the micelles. The lower $D$ value obtained for the $G 3$ copolymer, though not very significant, is again consistent with increased structural rigidity for the $G 3$ molecules as discussed earlier. In contrast, the initial diffusion coefficient $D_{\mathrm{i}}$ increases continuously from $G 1$ to $G 3$ copolymers, presumably because the indomethacin molecules diffuse predominantly out of the corona initially. While $D_{\mathrm{i}}$ increases with the generation number, the initial release rate decreases (Table 6.5). Theses two trends may sound contradictory, but they simply arise because the initial release rate varies inversely with the radius of a sphere 
(Equation 6.9), and the size of arborescent PS-g-P2VP copolymer micelles increases with the generation number (Table 6.5).

Table 6.5. Diffusion Coefficients of Indomethacin in Arborescent PS-g-P2VP Copolymers.

\begin{tabular}{|c|c|c|c|}
\hline & $G 1$ & $G 2$ & G3 \\
\hline${\operatorname{Radius~}(\mathrm{nm})^{\mathrm{a}}}$ & 12.5 & 22.5 & 39.5 \\
\hline$D_{i}\left(\mathrm{~cm}^{2} \mathrm{~s}^{-1}\right)^{\mathrm{b}}$ & $2.54 \times 10^{-18}(0.03)$ & $2.81 \times 10^{-18}(0.01)$ & $7.08 \times 10^{-18}(0.13)$ \\
\hline Average $D\left(\mathrm{~cm}^{2} \mathrm{~s}^{-1}\right)^{\mathrm{c}}$ & $1.25 \times 10^{-18}(0.18)$ & $4.67 \times 10^{-18}(0.07)$ & $4.33 \times 10^{-18}(0.08)$ \\
\hline Rate $\left(s^{-1}\right)$ at 2 hours ${ }^{d}$ & $2.06 \times 10^{-5}$ & $1.31 \times 10^{-5}$ & $1.19 \times 10^{-5}$ \\
\hline
\end{tabular}

\subsection{Conclusions}

The release of small molecules from arborescent copolymer micelles incorporating a branched polystyrene core and poly(2-vinylpyridine) segments in the shell was investigated. The release profiles obtained display sustained release characteristics with an initial burst, followed by slow approach to equilibrium. The diffusion coefficients for pyrene determined from fluorescence quenching experiments, and the average diffusion coefficients for indomethacin estimated by fitting an exact solution of Fick's second law of diffusion for a spherical device to the experimental data displayed the same increasing trend from $G 1$ to $G 2$, while a decrease was observed for the $G 3$ micelles. In spite of the increasing branching functionality of the copolymer micelles with the generation number, the results obtained 
from the fluorescence and the in vitro release studies are consistent with a more porous microstructure in acidified media for higher generation arborescent PS- $g$-P2VP copolymers up to G2. The increased number of P2VP chains per micelle for higher generation micelles presumably leads to enhanced electrostatic repulsions between the charged P2VP chains of higher generation arborescent PS-g-P2VP copolymers. Despite increased electrostatic repulsions, the average diffusion coefficient decreased for the $G 3$ copolymers, hinting at the dominating influence of branching functionality (branching density) on the structural rigidity of the molecules.

\subsection{Acknowledgements}

The financial support of the Natural Sciences and Engineering Research Council of Canada (NSERC) and the International Council for Canadian Studies (ICCS) are acknowledged with thanks. We also thank Prof. Jean Duhamel for providing access to the fluorescence characterization equipment.

\subsection{References}

1. Cowsar, D. R. In Controlled Release of Biologically Active Agents; Tanquary, A. C.; Lacey, R. E. Eds.; Plenum: New York, 1974, Chapter 1.

2. (a) Hayashi, T.; Yamazaki, T.; Yamaguchi, Y.; Sugibayashi, K.; Morimoto, Y. $J$. Controlled Release 1997, 43, 213. (b) Peracchia, M. T.; Gref, R.; Minamitake, Y.; Domb, A.; Lotan, N.; Langer, R. J. Controlled Release 1997, 46, 223. (c) Glavas-Dodov, M.; Goracinova, K.; Mladenovska, K.; Fredro-Kumbaradzi, E. Int. J. Pharm. 2002, 242, 
381. (d) DesNoyer, J. R.; McHugh, A. J. J. Controlled Release 2003, 86, 15. (e) Ferrero, C.; Bravo, I.; Jiménez-Castellanos, M. R. J. Controlled Release 2003, 92, 69.

3. (a) Tomaszewska, M.; Jarosiewicz, A. J. Agric. Food Chem. 2002, 50, 4634. (b) Shaviv, A.; Raban, S.; Zaidel, E. Environ. Sci. Techol. 2003, 37, 2251. (c) Mishra, S.; Bajpai, J.; Bajpai, A. K. J. Appl. Polym. Sci. 2004, 94, 1815.

4. (a) Tefft, J.; Friend, D. R. J. Controlled Release 1993, 27, 27. (b) Kenawy, E.-R.; Sakran, M. A. J. Appl. Polym. Sci. 2001, 80, 415.

5. (a) Fernández-Pérez, M.; González-Pradas, E.; Ureña-Amate, M. D. J. Agric. Food Chem. 1998, 46, 3828. (b) Kulkarni, A. R.; Soppimath, K. S.; Aminabhavi, T. M.; Dave, A. M.; Metha, M. H. J. Appl. Polym. Sci. 1999, 73, 2437. (c) Kumbar, S. G.; Aminabhavi, T. M. J. Appl. Polym. Sci. 2002, 84. 552.

6. (a) Kwon, G.; Naito, M.; Yokoyama, M.; Okano, T.; Sakurai, Y.; Kataoka, K. J. Controlled Release 1997, 48, 195. (b) Webber, S. E. J. Phys. Chem. B 1998, 102, 2618. (c) Teng, Y.; Morrison, M. E.; Munk, P.; Webber, S. E.; Procházka, K. Macromolecules 1998, 31, 3578. (d) Kim, S. Y.; Kim, J. H.; Kim, D.; An, J. H.; Lee, D. S.; Kim, S. C. J. Appl. Polym. Sci. 2001, 82, 2599. (e) Shen, E.; Kipper, M. J.; Dziadul, B.; Lim, M.-K.; Narasimhan, B. J. Controlled Release 2002, 82, 115.

7. (a) Liu, M.; Fréchet, J. M. J. Pharm. Sci. Technol. To. 1999, 2, 393. (b) Liu, H.; Jiang, A.; Guo, J.; Urich, K. E. J. Polym. Sci., Part A: Polym. Chem. 1999, 37, 703.

8. Liu, M.; Kono, K.; Fréchet, J. M. J. J. Controlled Release 2000, 65, 121.

9. Gauthier, M.; Li, J.; Dockendorff, J. Macromolecules 2003, 36, 2642.

10. Press, W. H.; Teukolsky, S. A.; Wetterling, W. T.; Flannery, B. P. Numerical Recipes in C: The Art of Scientific Computing; Cambridge University: Cambridge, 1992, p 683. 
11. Masuko, M.; Ohuchi, S.; Sode, K.; Ohtani, H.; Shimadzu, A. Nucleic Acids Res. 2000, 28, e34.

12. Lakowicz, J. R. Principles of Fluorescence Spectroscopy; Kluwer Academic/Plenum: New York, 1999, p 367.

13. (a) Cao, T.; Munk, P.; Ramireddy, C.; Tuzar, Z.; Webber, S. E. Macromolecules 1991, 24, 6300. (b) Štěpánek, M.; Krijtová, K.; Limpouchová, Z.; Procházka, K.; Teng, Y.; Munk, P.; Webber, S. E. Acta Polym. 1998, 49, 103.

14. Berlman, I. B. Energy Transfer Parameters of Aromatic Compounds; Academic: New York, 1973, p. 358.

15. Lakowicz, J. R. Principles of Fluorescence Spectroscopy; Kluwer Academic/Plenum: New York, 1999, Chapter 8

16. Bromberg, L.; Magner, E. Langmuir 1999, 15, 6792.

17. Claracq, J.; Santos, S. F. C. R.; Duhamel, J.; Dumouseaux, C.; Corpart, J.-M. Langmuir 2002, $18,3829$.

18. Hrdlovič, P.; Lukáč I. J. Photochem. Photobiol. A: Chem. 2000, 133, 73.

19. (a) Štěpánek, M.; Krijtová, K.; Procházka, K.; Teng, Y.; Webber, S. E.; Munk, P. Acta Polym. 1998, 49, 96. (b) Clements, J. H.; Webber, S. E. Macromolecules 2004, 37, 1531.

20. Frank, R. S.; Merkle, G.; Gauthier, M. Macromolecules 1997, $30,5397$.

21. Kee, R. A.; Gauthier, M. Macromolecules 2002, 35, 6526.

22. Sauer, M.; Meier, W. In Colloids and Colloid Assemblies; Caruso, F. Ed.; Wiley-VCH: Weinheim, 2004, p 160.

23. Clements, J. H.; Webber, S. E. J. Phys. Chem. B 1999, 103, 9366. 
24. (a) Kumbar, S. G.; Kulkarni, A. R.; Dave, A. M.; Aminabhavi, T. M. J. Hazard. Mater. 2002, B89, 233. (b) Kumbar, S. G.; Dave, A. M.; Aminabhavi, T. M. J. Appl. Polym. Sci. 2003, 90,451 .

25. Kim, S. Y.; Shin, I. G.; Lee, Y. M.; Cho, C. S.; Sung, Y. K. J. Controlled Release 1998, $51,13$.

26. (a) Taylor, L. S.; Zografi, G. Pharm. Res. 1997, 14, 1691. (b) Forster, A.; Hempenstall, J.; Rades, T. Internet J. Vib. Spec. 2001, 5, 6.

27. Siepmann, J.; Peppas, N. A. Adv. Drug Delivery Rev. 2001, 48, 139.

28. Aminabhavi, T. M.; Harlapur, S. F.; Balundgi, R. H.; Ortego, J. D. J. Appl. Polym. Sci. 1996, 59, 1857.

29. Crank, J. The Mathematics of Diffusion, Clarendon: Oxford, 1975, p 91.

30. Baker, R.W.; Lonsdale, H. K. In Controlled Release of Biologically Active Agents; Tanquary, A. C.; Lacey, R. E. Eds.; Plenum: New York, 1974, p 46. 
Chapter 7

General Conclusions and Suggestions for Future Work 


\subsection{General Conclusions}

Amphiphilic arborescent copolymers are dendritic macromolecules with unusual physical properties reminiscent of both sterically stabilized colloids and unimolecular micelles. It appeared that these materials could be useful as thin films and in the solubilization and controlled release of small molecules, but their interfacial properties remained so far essentially unexplored. The aim of this dissertation was to start exploring the interfacial properties of these materials by establishing structure-property relations for their self assembly at the air-water interface, as well as for their small molecule solubilization and release characteristics.

It was thus demonstrated that 'crew-cut' micelles of arborescent polystyrene-graftpoly(ethylene oxide) copolymers with short hydrophilic segments self-assembled at the airwater interface. In contrast, arborescent polystyrene-graft-poly(2-vinylpyridine) copolymers remained as unimolecular (non-associated) micellar species in solution and were able to solubilize and release hydrophobic probe molecules in a controlled fashion.

Arborescent PS-g-PEO copolymers spontaneously formed one- and two-dimensional superstructures at the air-water interface. The topology of the Langmuir-Blodgett films transferred on cleaved mica showed a strong dependence on the composition and the structure of the copolymers. Copolymers with low PEO contents $(\leq 15 \% \mathrm{w} / \mathrm{w})$ formed large island-like clusters, while copolymers with higher PEO contents $(\geq 22 \% \mathrm{w} / \mathrm{w})$ formed ribbon-like superstructures, the low branching functionality samples forming longer ribbons. Atomic Force Microscopy (AFM) image analysis suggested that at low compressions, the molecules were in a gas-like phase with the PEO chains adsorbed at the water subphase, in analogy to linear and star-branched PS-PEO block copolymers. At very high compressions, 
however, the PEO chains apparently desorbed into the water subphase in a brush-like conformation. The formation of these superstructures was favored to some extent by compression and increases in subphase temperature. Subjecting the copolymer solutions to partial degradation with UV radiation, in particular, was shown to be a versatile method to tailor the association behavior of the copolymers and the type of superstructures formed.

The solubilization and release properties of arborescent PS-g-P2VP copolymer micelles were also investigated and found to depend on the architecture of both the copolymer and the solute. For example, highly hydrophobic probes like pyrene were solubilized predominantly within the PS core while less hydrophobic molecules such as 1methylnaphthalene were solubilized in the whole molecule. The solubilization capacity was also higher for smaller solutes than for larger and more symmetrical solutes. The release profiles obtained displayed sustained release characteristics with an initial burst in release and a much more gradual attainment of equilibrium conditions. The diffusion coefficients for pyrene determined by fluorescence, and the average diffusion coefficients for indomethacin estimated from Fick's second law of diffusion displayed the same trend, i.e. an increase from $G 1$ to $G 2$, followed by a decrease for G3. This trend was attributed to increasing microporosity of the shell due to enhanced electrostatic repulsions, in competition with structural stiffening (dominant in the G3 copolymers) leading to lower diffusion coefficients.

\subsection{Suggestions for Future Work}

The present research was the very first investigation of the behavior of arborescent PS-g-PEO amphiphilic copolymers at the air-water interface. While these neutral dendritic micelles have already yielded some unexpected results, it would certainly be interesting to 
examine the behavior of amphiphilic arborescent polyelectrolytes such as protonated arborescent PS-g-P2VP copolymers or poly(methacrylic acid) copolymers, as the film topology of these materials should be influenced not only by copolymer composition and structure, but also by other parameter such as the $\mathrm{pH}$, the neutralization level, etc.

\subsubsection{Behavior of Arborescent PS-g-P2VP Copolymers at the Air-water}

Interface. In a preliminary investigation using arborescent copolymer sample G1PS-P2VP513, stable Langmuir monolayers were obtained (as indicated by the shape of the compression isotherms) at the air-water interface. The resulting Langmuir-Blodgett films incorporated exclusively close-parked individual molecules without any indication of aggregation (Figure 7.1a). The copolymer used had a PS core branching functionality $f_{w}=64$ and an overall $f_{w}=$ 630. The topology of the films changed to ribbon-like or pearl necklace-like superstructures (Figure 7.1b) when the overall branching functionality was decreased to 370 (sample G1PSP2VP5-20, obtained by grafting fewer P2VP chains on the polystyrene substrate).

Because the arborescent PS- $g$-P2VP copolymers are polyelectrolyte precursors, their behavior at the air-water interface and in solution should prove to be very interesting to investigate. Variations in the $\mathrm{pH}$ and the ionic strength of either the copolymer solution or the subphase may be a very powerful method to control the assembly process in these systems. This is because these parameters should provide control over the magnitude of electrostatic repulsive forces between the P2VP segments, and therefore over the extent of electrosteric (electrostatic and steric) stabilization of the molecules. 

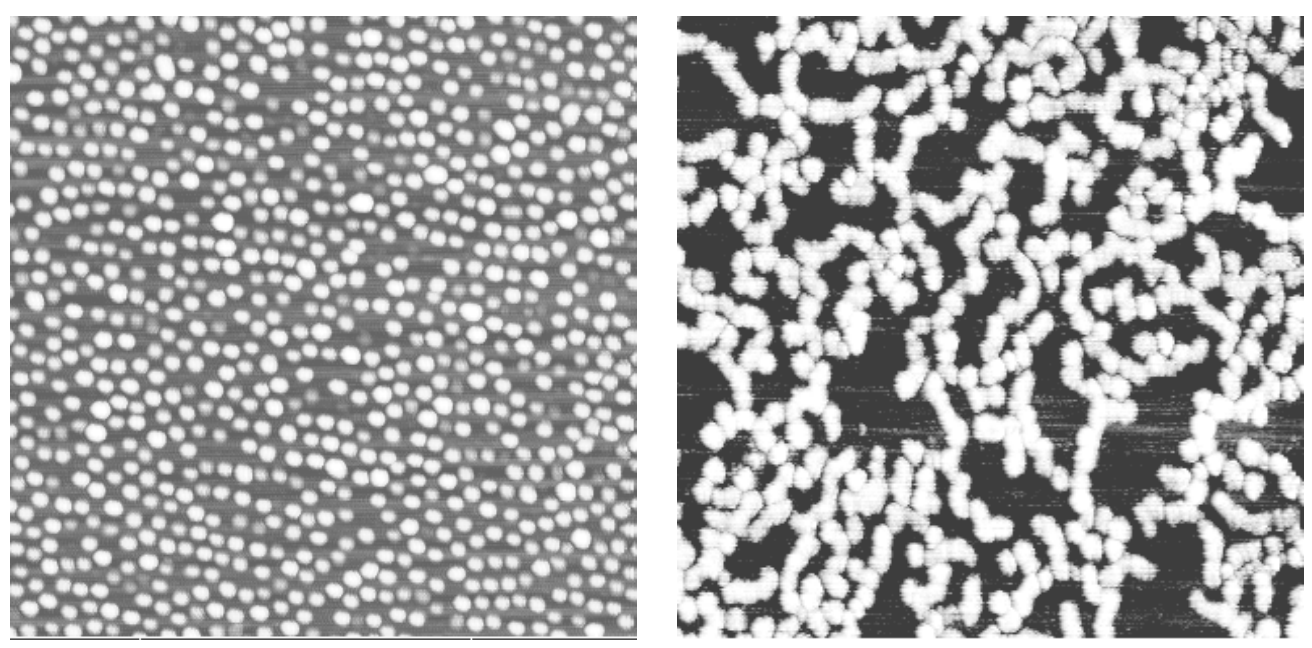

Figure 7.1. AFM micrographs for Langmuir-Blodgett films of arborescent PS-g-P2VP copolymers before compression, subphase temperature $=22.0 \pm 0.5^{\circ} \mathrm{C}$ : (a) $G 1 \mathrm{PS}-g$-P2VP, $f_{\mathrm{w}}$ $=630,(\mathrm{~b})$ G1PS- $g$-P2VP, $f_{\mathrm{w}}=370$.

\subsubsection{Aggregation of Arborescent PS-g-P2VP Copolymers in Aqueous Solutions.}

Block copolymers are known to form superstructures with different morphologies in mixed solvent systems, where one of the solvents present is selective for one of the blocks. The same technique could be applied to arborescent copolymers to study the influence of solvent composition and copolymer structure on the self-assembly of amphiphilic molecules in solution. Interesting results were obtained during preliminary investigations using arborescent copolymer sample G1PS-P2VP5-20. The micelles were first dissolved in THF (2.5 mg/mL), a common solvent for both blocks. Dilute aqueous $\mathrm{HCl}(0.05 \mathrm{M}$, ca. $0.75 \mathrm{~mL})$ was then added dropwise to reach the cloud point, and the solution was dialyzed against milli-Q water. Pictures obtained by transmission electron microscopy (TEM) after staining the samples with iodine vapor for about 4 hours (Figure 7.2) showed that the molecules formed large spherical compound micelles with sizes and morphologies depending on the 
$\mathrm{HCl} / 2 \mathrm{VP}$ mole ratio $\left(R_{\mathrm{m}}\right)$ used in the experiments. At $R_{\mathrm{m}}=38$, mostly individual molecules were observed with diameters of $40 \pm 2 \mathrm{~nm}$ (Figure 7.2a). As $R_{\mathrm{m}}$ was decreased, the molecules started assembling into larger, poorly defined superstructures but interestingly, superstructures of uniform size were obtained at $R_{\mathrm{m}}=4.2$.
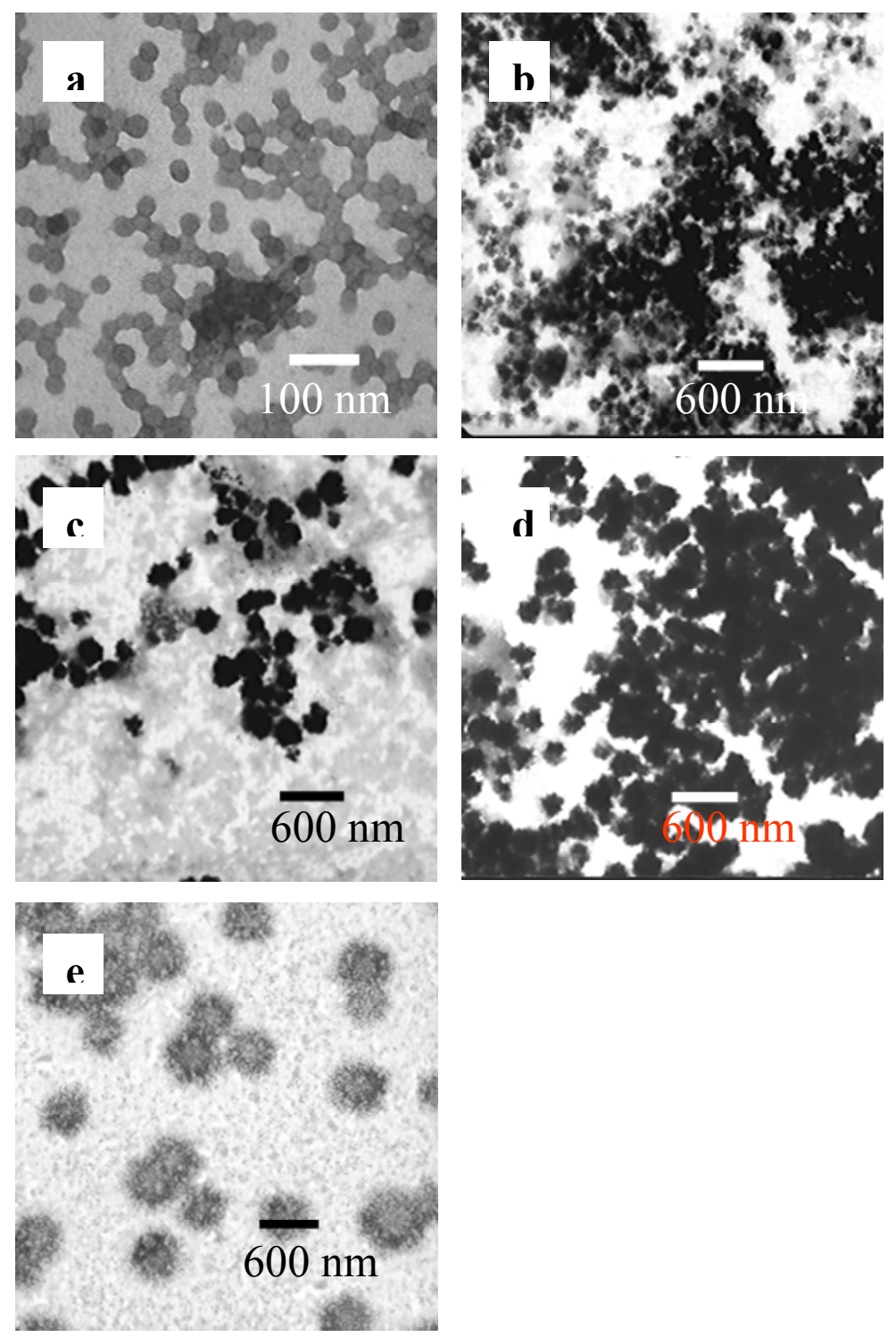

Figure 7.2. Change in micellar aggregate size with decreasing $\mathrm{HCl} / 2 \mathrm{VP}$ mole ratio $R_{\mathrm{m}}$. (a) $R_{\mathrm{m}}=38$, (b) $R_{\mathrm{m}}=16.7$, (c) $R_{\mathrm{m}}=12.5$, (d) $R_{\mathrm{m}}=8.3$, (e) $R_{\mathrm{m}}=4.2$. 
The average diameter of the aggregates apparently varied with $R_{\mathrm{m}}$ according to a power law model (Figure 7.3), to reach a value of $652 \pm 5 \mathrm{~nm}$ for $R_{\mathrm{m}}=4.2$. Another interesting detail is that no aggregation was observed in any of the solutions based on dynamic light scattering measurements, suggesting that the self assembly occurred during solvent evaporation on the TEM grid. Even upon doubling the concentration of the copolymer in solution through solvent evaporation, the hydrodynamic diameter of the molecules was unchanged at $51 \pm 5$ nm.

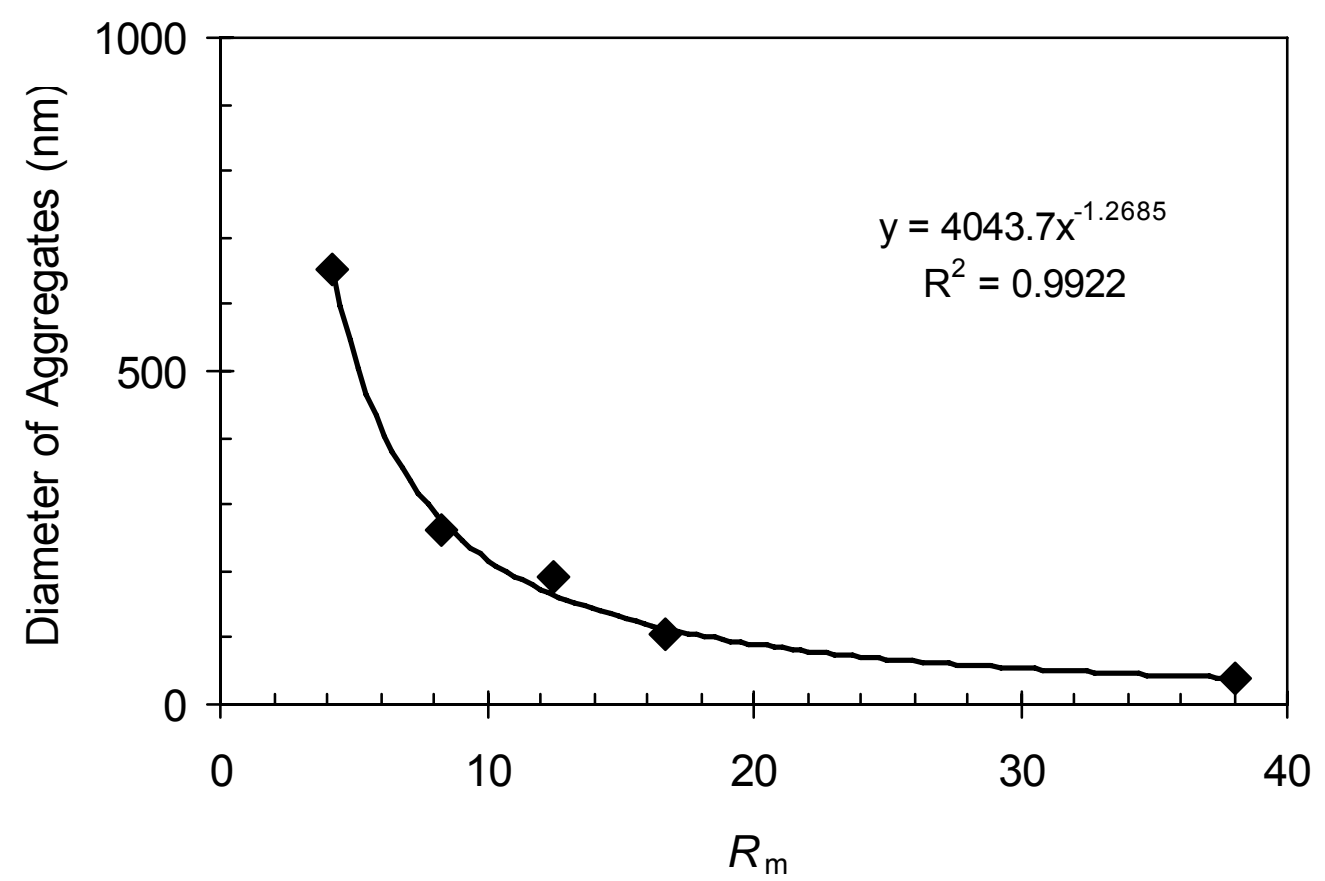

Figure 7.3. Dependence of average diameter of large compound micelles on $\mathrm{HCl} / 2 \mathrm{VP}$ mole ratio $R_{\mathrm{m}}$.

From these preliminary results, it is difficult to ascertain whether aggregation was due to a decrease in the degree of ionization as $R_{\mathrm{m}}$ was reduced, or to changes in ionic strength and to the enormous increase in copolymer concentration as the solutions evaporated on the 
copper grids during TEM sample preparation. A systematic investigation of the influence of ionic strength and copolymer concentration would be required to improve understanding of the self-assembly of these arborescent PS-g-P2VP copolymers in solution. The work could be subsequently expanded to examine the influence of copolymer composition and architecture, and to other polyelectrolytes such as arborescent polystyrene-graftpoly(methacrylic acid) copolymers. 